\title{
BEHAVIOR-BASED PRICE DISCRIMINATION AND PRODUCT CHOICE
}

\author{
Chongwoo Choe \\ Noriaki Matsushima
}

March 2020

The Institute of Social and Economic Research

Osaka University

6-1 Mihogaoka, Ibaraki, Osaka 567-0047, Japan 


\title{
Behavior-Based Price Discrimination and Product Choice*
}

\author{
Chongwoo Choe \\ Centre for Global Business, Monash University \\ Department of Economics, Monash University \\ chongwoo.choe@monash.edu \\ Noriaki Matsushima $^{\dagger}$ \\ ISER, Osaka University \\ nmatsush@iser.osaka-u.ac.jp
}

March 9, 2020

\begin{abstract}
We study a two-period model of behavior-based price discrimination in Fudenberg and Tirole (2000) but allow firms to make product choice in the first period. We show that the only possible equilibrium involves maximal differentiation. This is in contrast to Choe et al. (2018) where equilibrium features less than maximal differentiation when competition is in personalized pricing. Thus, our result highlights an important interplay between the type of price competition and product choice.
\end{abstract}

Keywords: Behavior-based price discrimination, spatial competition JEL Classification Number: D43, L13

${ }^{*}$ We gratefully acknowledge financial support from the JSPS KAKENHI (Grant Numbers JP15H03349, JP15H05728, JP17H00984, JP18H00847, and JP19H01483), the JSPS Invitation Fellowship (S16713), the International Joint Research Promotion Program at Osaka University, and the Murata Science Foundation. The usual disclaimer applies.

${ }^{\dagger}$ The corresponding author. Institute of Social and Economic Research, Osaka University, 6-1 Mihogaoka, Ibaraki, Osaka 567-0047, Japan. Email: nmatsush@iser.osaka-u.ac.jp 


\section{Introduction}

Behavior-base price discrimination (BBPD) refers to the practice whereby firms condition their price offers on customers' purchase histories. The advances in technologies have lowered the costs of firms' investment in customer information, leading to proliferation of various types of BBPD. ${ }^{1}$ In the simplest two-period model of BBPD, firms segment a market into two based on the first-period purchase, existing customers and new customers, and exercise third-degree price discrimination in the second period.

Existing studies on BBPD typically focus only on the pricing game assuming product differentiation is exogenously fixed. For example, Fudenberg and Tirole (2000) and many subsequent studies consider a two-period Hotelling model taking maximal differentiation as given. While maximal differentiation is an equilibrium outcome in the static Hotelling model with quadratic transportation cost, it is not obvious whether it continues to be so in the dynamic context. Suppose a firm chooses an interior location in the first period while its rival chooses the opposite end. In the second period, the first firm is in a more strategic position than its rival, which it may be able to leverage and poach the rival's customers more effectively.

This line of argument suggests that maximal differentiation may not be an equilibrium outcome when locations are chosen in the first period. Indeed this is shown by Choe et al. (2018): when firms choose locations in the first period and compete in personalized pricing in the second period, BBPD results in equilibrium where one firm chooses an interior location. But we are not aware of any study that endogenizes location choice when the second-period competition is in third-degree price discrimination. The purpose of our study is to fill this gap.

Our main result is that, unlike Choe et al. (2018), maximal differentiation is the only possible equilibrium outcome. The intuition is as follows. When the second-period competition is in third-degree price discrimination, the firm with a larger market share loses more customers to its rival. The reason is that, with third-degree price discrimination, a firm has to charge the same price to all its loyal customers, some of whom are inevitably closer to the rival when the firm has a larger market share. Thus the second period does not matter much to the firm's decision in the first period. As a result, firms choose maximal differentiation to soften competition in the first period.

Given that the result in Choe et al. (2018) is based on competition in personalized pricing, our result is driven mainly by third-degree price discrimination. This highlights an important interplay between the type of price competition and product choice. We proceed below by providing a brief literature review, followed by the model and analysis, and some discussions and conclusion.

\footnotetext{
${ }^{1}$ See Ezrachi and Stucke (2016) for various examples of BBPD.
} 


\section{Related Literature}

The first strand of literature on BBPD shows that BBPD generally lowers firm profitability by intensifying competition, unless there are sufficient asymmetries at firm- or consumer-level. $^{2}$ This is true whether firms compete in third-degree price discrimination (Chen, 1997; Villas-Boas, 1999; Fudenberg and Tirole, 2000; Pazgal and Soberman, 2008; Esteves, 2010) or in personalized pricing (Zhang, 2011; Choe et al., 2018).

The second strand of literature introduces various asymmetries and shows how BBPD can improve profitability. Such asymmetries include enhanced services that firms can offer only to loyal customers (Acquisti and Varian, 2005; Pazgal and Soberman, 2008), quality difference between firms (Jing, 2017; Rhee and Thomadsen, 2017), asymmetry in consumer preferences (Chen and Zhang, 2009; Shin and Sudhir, 2010), or consumers' fairness concern (Li and Jain, 2016).

Our paper belongs to the first strand. The general intuition behind the competitionintensifying effect of BBPD is that customer information made available from past purchases makes firms more aggressive in pricing. Choe et al. (2018) further shows that firms also make more aggressive product choice when competition is in personalized pricing. ${ }^{3}$ But all the above studies that assume third-degree price discrimination take exogenously fixed product choice as given. Thus it remains unanswered if the result in Choe et al. (2018) continues to hold when competition is in third-degree price discrimination. Our paper addresses this question.

\section{The Model}

Our model is an extension of Fudenberg and Tirole (2000) where we incorporate location choice by firms. There are two periods, $\tau=1,2$. Consumers are located uniformly over a Hotelling linear city $[0,1]$, and each consumer's location stays the same over the two periods. Each consumer buys one unit of good in each period and derives utility $v$ from each unit. We assume $v$ is sufficiently large so that the entire market is covered in equilibrium. Two firms, $i=A, B$, have the same constant marginal cost of production, which is normalized to zero. Consumers have quadratic transportation costs: if firm $i$ is located at $a$ and sets a price $p_{i}$, then consumer $x$ gets a surplus of $v-p_{i}-t(x-a)^{2}$ by purchasing from firm $i$.

In $\tau=1$, competition follows the standard Hotelling model. Firms simultaneously

\footnotetext{
${ }^{2}$ See Chen (2005) or Fudenberg and Villas-Boas $(2006,2012)$ for more comprehensive reviews.

${ }^{3}$ Zhang (2011) considers a two-period model with personalized pricing in the second period, but allows costless personalization of products as well as prices. Thus she departs from the standard BBPD assumption that price is the only choice variable in the second period. Her assumption of product personalization leads to substantially different results from ours. For example, there is no customer poaching in Zhang (2011) in contrast to ours.
} 
choose locations which are fixed over two periods, after which they compete in price. Let $\mathcal{A}(\mathcal{B})$ be the set of consumers that choose firm $A(B)$ in $\tau=1$. In $\tau=2$, firms compete using third-degree price discrimination where each firm chooses two prices, one for its $\tau=1$ customers and the other for its rival's $\tau=1$ customers, the latter we call the poaching price.

In making $\tau=1$ decisions, firms discount $\tau=2$ profits by $\delta_{f} \in[0,1]$ and consumers discount $\tau=2$ surplus by $\delta_{c} \in[0,1]$. As shown in Choe et al. (2018), however, solving the game for general discount factors is not possible even in the simpler case where the $\tau=2$ competition is in personalized pricing. Accordingly, for our main result (Proposition 1), we follow Fudenberg and Tirole (2000) and assume the common discount factor $\delta_{c}=\delta_{f}=\delta \in[0,1]$. For additional results (Propositions 2, 3), we follow Choe et al. (2018) and assume consumers are myopic in that $\delta_{c}=0$.

\section{Analysis}

Fix firm $A$ 's location at $a$ and firm $B$ 's location at $b$ and, without loss of generality, assume $0 \leq a \leq b \leq 1$. Let $z$ be the marginal consumer in $\tau=1$ who is indifferent between choosing either firm in $\tau=1$. For $i=A, B$, denote firm $i$ 's $\tau=1$ price by $p_{i}$, its $\tau=2$ price for its own $\tau=1$ customers by $p_{i o}$, and its $\tau=2$ poaching price by $p_{\text {in }}$. Thus in $\tau=2$, firm $A$ chooses $p_{A o}$ for consumers in $\mathcal{A}$ and $p_{A n}$ for consumers in $\mathcal{B}$. Similarly firm $B$ chooses $p_{B o}$ for consumers in $\mathcal{B}$ and $p_{B n}$ for consumers in $\mathcal{A}$. We solve the game backwards.

\subsection{Second period}

Given the $\tau=1$ marginal consumer $z$, we have $\mathcal{A}=[0, z]$ and $\mathcal{B}=[z, 1]$. In each set, there may be consumers who want to switch to a new firm in $\tau=2$. Let $z_{A} \in \mathcal{A}$ be a marginal consumer such that consumers in $\left[0, z_{A}\right]$ continue to choose firm $A$ while those in $\left[z_{A}, z\right]$ switch to firm $B$. Then $z_{A}$ satisfies

$$
p_{A o}+t\left(z_{A}-a\right)^{2}=p_{B n}+t\left(z_{A}-b\right)^{2}
$$

which leads to

$$
z_{A}=\frac{\left(b^{2}-a^{2}\right) t-\left(p_{A o}-p_{B n}\right)}{2(b-a) t} .
$$

Similarly let $z_{B}$ be the marginal consumer in $\mathcal{B}$, i.e., $p_{A n}+t\left(z_{B}-a\right)^{2}=p_{B o}+t\left(z_{B}-b\right)^{2}$. Then we have

$$
z_{B}=\frac{\left(b^{2}-a^{2}\right) t-\left(p_{A n}-p_{B o}\right)}{2(b-a) t} .
$$


Firms' $\tau=2$ profits are

$$
\begin{aligned}
& \pi_{A 2}=p_{A o} z_{A}+p_{A n}\left(z_{B}-z\right) \\
& \pi_{B 2}=p_{B n}\left(z-z_{A}\right)+p_{B o}\left(1-z_{B}\right) .
\end{aligned}
$$

Firm $i$ chooses $p_{i o}$ and $p_{i n}$ to maximize $\pi_{i 2}$. Solving the first-order conditions simultaneously, we have

$$
\begin{aligned}
& p_{A o}=\frac{(b-a)(2 z+a+b) t}{3}, \quad p_{A n}=\frac{(b-a)(2+a+b-4 z) t}{3}, \\
& p_{B o}=\frac{(b-a)(4-2 z-a-b) t}{3}, p_{B n}=\frac{(b-a)(4 z-a-b) t}{3} .
\end{aligned}
$$

Plugging these prices back into $z_{A}$ and $z_{B}$ above, we obtain

$$
z_{A}=\frac{a+b+2 z}{6}, \quad z_{B}=\frac{2+a+b+2 z}{6} .
$$

Note that $z_{A} \leq z$ if and only if $z \geq(a+b) / 4$; if $z \leq(a+b) / 4$, we need to consider a corner solution $z_{A}=z$. Similarly, $z_{B} \geq z$ if and only if $z \leq(2+a+b) / 4$; otherwise, we need to consider the case $z_{B}=z$. Thus there are three possibilities in $\tau=2$ : (i) when $z=z_{A}$, firm $B$ cannot poach any of firm $A$ 's customers and the only possibility is one-way poaching by firm $A$; (ii) when $z_{A}<z<z_{B}$, there is two-way poaching; (iii) when $z_{B}=z$, the only possibility is one-way poaching by firm $B$. In the proof of Proposition 1, we show that only case (ii) is possible in equilibrium. Thus we will focus our discussion on this case, of which condition can be stated as

$$
\frac{a+b}{4}<z<\frac{2+a+b}{4}
$$

Substituting the above prices, $z_{A}$ and $z_{B}$ into each firm's $\tau=2$ profit, we have

$$
\begin{aligned}
& \pi_{A 2}=\frac{t(b-a)\left(2+2 a+a^{2}+2 b+2 a b+b^{2}-8 z-2 a z-2 b z+10 z^{2}\right)}{9}, \\
& \pi_{B 2}=\frac{t(b-a)\left(8-4 a+a^{2}-4 b+2 a b+b^{2}-8 z-2 a z-2 b z+10 z^{2}\right)}{9} .
\end{aligned}
$$

To see how market shares change in $\tau=2$, let us denote firm $i$ 's market share in $\tau=2$ by $S_{i}$, the fraction of consumers switching from firm $A$ to firm $B$ by $S_{A \rightarrow B}$, and the fraction switching the other way by $S_{B \rightarrow A}$. Consumers in $\left[z_{A}, z\right]$ switch from firm $A$ to firm $B$, hence $S_{A \rightarrow B}=z-z_{A}=(4 z-a-b) / 6$. On the other hand, firm $A$ serves new consumers in $\left[z, z_{B}\right]$ who switch from firm $B$ to firm $A$, leading to $S_{B \rightarrow A}=z_{B}-z=$ $(2-4 z+a+b) / 6$. As a result, the $\tau=2$ market shares become $S_{A}=z+S_{B \rightarrow A}-S_{A \rightarrow B}=$ $(1+a+b-z) / 3$ and $S_{B}=(1-z)-S_{B \rightarrow A}+S_{A \rightarrow B}=(2-a-b+z) / 3$.

Fudenberg and Tirole (2000) consider the case with $a=0$ and $b=1$. In this case, 
it is easy to see $S_{A \rightarrow B} \geq S_{B \rightarrow A}$ and $S_{A} \leq S_{B}$ if and only if $z \geq 1 / 2$. Thus the firm with a larger market share in $\tau=1$ loses more customers and ends up having a smaller market share in $\tau=2$. Moreover, one can check $\pi_{A 2}=\pi_{B 2}$ for all $z$ when $a=0$ and $b=1$. In sum, there is no benefit in having a larger market share in $\tau=1$ due to the two-way customer switching in $\tau=2$. This is why the equilibrium in Fudenberg and Tirole (2000) is symmetric with $z=1 / 2$.

Similar reasoning applies to our case. Suppose firm $B$ chooses $b=1$ but firm $A$ chooses an interior location $a>0$ and secures a larger market share in $\tau=1$. But this increases poaching by firm $B$, which has a negative effect on firm $A$ 's $\tau=2$ market share. Moreover, less than maximal differentiation intensifies competition in both periods. These discussions imply that firms do not have strong incentives to secure a larger market share in $\tau=1$, which in turn reduces their incentives to choose aggressive locations. Thus we may conjecture that maximal differentiation becomes an equilibrium outcome, which we confirm below.

\subsection{First period}

Since consumers are forward-looking, the marginal consumer's location in $\tau=1$ depends on the outcome consumers anticipate in $\tau=2$. From the previous analysis, we know there are three possibilities: (i) $z=z_{A}$, (ii) $z_{A}<z<z_{B}$, and (iii) $z_{B}=z$.

As before, we focus on the case with two-way poaching in $\tau=2$ : $z_{A} \leq z \leq z_{B}$. If consumers anticipate this outcome, then the $\tau=1$ marginal consumer $z$ is indifferent between choosing firm $A$ in $\tau=1$ but switching to firm $B$ in $\tau=2$, and choosing firm $B$ in $\tau=1$ but switching to firm $A$ in $\tau=2$. Given consumers' discount factor $\delta_{c}, z$ is then given by

$$
p_{A}+t(z-a)^{2}+\delta_{c}\left(p_{B n}+t(z-b)^{2}\right)=p_{B}+t(z-b)^{2}+\delta_{c}\left(p_{A n}+t(z-a)^{2}\right) .
$$

Thus the marginal consumer's location in this case is

$$
z=\frac{(b-a) t\left((b+a)\left(3-\delta_{c}\right)+2 \delta_{c}\right)-3\left(p_{A}-p_{B}\right)}{2\left(3+\delta_{c}\right)(b-a) t} .
$$

Given firms' discount factor $\delta_{f}$, each firm's total discounted profit is

$$
\begin{aligned}
& \Pi_{A}=p_{A} z+\delta_{f} \pi_{A 2}, \\
& \Pi_{B}=p_{B}(1-z)+\delta_{f} \pi_{B 2} .
\end{aligned}
$$

Of course, the $\tau=1$ marginal consumer's location will change if consumers anticipate different outcomes in $\tau=2$. This will in turn lead to different total discounted profit for each firm. This complicates the analysis of the $\tau=1$ game. We will first outline how to 
solve for the equilibrium of the $\tau=1$ game. We will then discuss the complications in solving for the equilibrium.

The $\tau=1$ game consists of two stages: firms choose $a$ and $b$ first, which is followed by the choice of $p_{A}$ and $p_{B}$. We solve the game backwards. Given $(a, b)$, we first solve for the equilibrium of the pricing game denoted by $p_{A}^{*}(a, b), p_{B}^{*}(a, b)$, hence the marginal consumer's location denoted by $z^{*}(a, b)$. We then substitute these back into total discounted profit functions, denoted by $\Pi_{A}^{*}(a, b)$ and $\Pi_{B}^{*}(a, b)$, and find equilibrium locations $\left(a^{*}, b^{*}\right)$ by solving simultaneously $\partial \Pi_{A}^{*}\left(a^{*}, b^{*}\right) / \partial a \leq 0$ with equality if $a^{*}>0$, and $\partial \Pi_{B}^{*}\left(a^{*}, b^{*}\right) / \partial b \geq 0$ with equality if $b^{*}<1$. Finally we substitute $\left(a^{*}, b^{*}\right)$ into $p_{A}^{*}(a, b), p_{B}^{*}(a, b),(1),(2),(3)$, and (4) to find the equilibrium of the whole game.

The main difficulty in solving for the $\tau=1$ equilibrium arises from the fact that there are three possible outcomes in $\tau=2$. Corresponding to each outcome, we have a different profit function for each firm. Thus each firm's reaction function in the pricing stage consists of three possibly discontinuous pieces. This means that, following location choice by one firm, we need to consider all possible deviations by the other firm including those that lead to points on the different pieces of the reaction function.

When the $\tau=2$ competition is in personalized pricing as in Choe et al. (2018), there are only two possible outcomes with one-way poaching in $\tau=2$. This is because a firm can use personalized pricing to protect its turf effectively unless its market share is too large. In contrast, third-degree price discrimination is a blunt tool to protect one's turf. It is because, with third-degree price discrimination, a firm has to charge the same price to all its loyal customers. Thus competition 'around the middle' cannot be too tough, leading to two-way poaching as a possible outcome. With three possible outcomes, the analysis becomes significantly more complicated.

Moreover, as shown in Choe et al. (2018), finding the $\tau=1$ equilibrium for general discount factors is not possible even when competition is in personalized pricing in $\tau=2$. In addition, pure-strategy equilibria do not exist when both firms are located sufficiently close to one end in that $a+b \approx 2$ or $a+b \approx 0$. Since our case with third-degree price discrimination involves more complicated analysis than with personalized pricing, we also expect non-existence of pure-strategy equilibria in some cases.

We present two results below. First, maximal differentiation is a unique equilibrium under certain conditions on discount factors and $a+b$. Second, if firms' discount factor is $\delta_{f}=\delta \in[0,1]$ while consumers are myopic in that $\delta_{c}=0$, then maximal differentiation is a unique equilibrium for all $\delta .{ }^{4}$ The full analysis is long and tedious. So we only provide a sketch of the proof in the appendix while referring to the technical appendix for the complete proof.

\footnotetext{
${ }^{4}$ Choe et al. (2018, Proposition 5) considered the case where $\delta_{f}=1$ and $\delta_{c}=0$. In contrast to our result, they obtained two asymmetric equilibria with less-than-maximal differentiation.
} 
Proposition 1. Suppose $\delta_{c}=\delta_{f}=\delta \in[0,1]$. Then there is a unique equilibrium with maximal differentiation (i) if $\delta<0.826$ or (ii) for all $\delta \in[0,1]$ if $a+b$ is restricted to the range $[0.415,1.585]$. The equilibrium is given by $a=0, b=1, p_{A}=p_{B}=(3+\delta) t / 3$, $p_{A o}=p_{B o}=(2 t) / 3, p_{A n}=p_{B n}=t / 3$, and $z=1 / 2, z_{A}=1 / 3, z_{B}=2 / 3$.

Proof. See the appendix.

The reason for the sufficient conditions stated in Proposition 1 is as follows. Suppose firms choose locations close enough to each other at one end of the market in that $a+b$ is close to 0 or 2 . Then the $\tau=2$ outcome is more likely to involve one-way poaching. For example, if $a+b$ is close to 2 , then in $\tau=2$, firm $B$ is likely to poach firm $A$ 's customers. If firm $B$ values its $\tau=2$ profit sufficiently, i.e., large $\delta$, then it has incentives to cut its $\tau=1$ price further to secure a more advantageous location in $\tau=2$. This destabilizes the pricing equilibrium in $\tau=1$.

Our next result shows that if consumers are myopic in that $\delta_{c}=0$, then the above incentives disappear, leading to the equilibrium with maximal differentiation for all values of firms' discount factor. The intuition is that, as consumers become myopic, their $\tau=1$ decisions do not depend much on favorable poaching offers in $\tau=2$. This makes their $\tau=1$ demands more price elastic, which can intensify price competition in $\tau=1$. Thus firms benefit from choosing differentiation to soften the price competition in $\tau=1$.

Proposition 2. Suppose $\delta_{c}=0$. Then for all $\delta_{f}=\delta \in[0,1]$, the game has a unique equilibrium described in Proposition 1 with the only difference $p_{A}=p_{B}=t$.

Proof. From the proof of Proposition 1, one can show that, if $\delta_{c}=0$, then only the first-period pricing equilibrium corresponding to the two-way poaching outcome exists for all $\delta_{f}=\delta \in[0,1]$ and $a, b \in[0,1]$. The details are in the technical appendix.

\section{Discussions}

\subsection{Comparison with Choe et al. (2018)}

Our result of maximal differentiation differs from that in Choe et al. (2018). The difference stems from the difference in the way firms compete in the second period. Competition is in personalized pricing in Choe et al. (2018) whereas it is in third degree price discrimination in our case. We discuss below why firms' product choice hinges on the type of price competition that follows.

When competition is in personalized pricing, firms compete under asymmetric information: a firm knows more about its own past customers than about its rival's past 
customers. Since personalized pricing allows a firm to extract surplus from its own customers more effectively than uniform price, the ability to exercise personalized pricing creates incentives for firms to secure a large market share. This intensifies competition in the first period, leading to less than maximal differentiation.

When competition is in third-degree price discrimination, however, firms compete under minimal, symmetric information: two firms share identical information about which market segment made purchase from each firm in the first period. As discussed previously, uniform pricing based on symmetric information undermines the benefits of having a large market share because of customer switching in the second period. Given quadratic transportation costs, firms then have more incentives to soften competition in the first period than compete for larger profit in the second period. Thus firms choose maximal differentiation.

\subsection{Welfare}

In this section, we discuss welfare implications of BBPD. Given that the market is fully covered, social optimum depends only on the average distance traveled by a consumer. Then it follows that the optimal location choice involves $a=1 / 4, b=3 / 4$ with average distance traveled equal to $1 / 8$. Thus in $\tau=1$, there is too much differentiation as in the standard Hotelling equilibrium where the average distance traveled is $1 / 4$. The $\tau=2$ equilibrium is the same as that in Fudenberg and Tirole (2000): firm $A$ continues to serve customers in $[0,1 / 3]$ while those in $[1 / 3,1 / 2]$ switch to firm $B$; firm $B$ continues to serve customers in $[2 / 3,1]$ while those in $[1 / 2,2 / 3]$ switch to firm $A$. Due to such inefficient customer switching, welfare is even lower than the Hotelling equilibrium. The average distance traveled in $\tau=2$ is equal to $11 / 36>1 / 4 .^{5}$

Firms also have lower profits due to BBPD compared to the Hotelling equilibrium. For simplicity, suppose $\delta_{c}=\delta_{f}=\delta$ as in Fudenberg and Tirole (2000). In the Hotelling equilibrium adapted to our setting, each firm earns profit equal to $t / 2$ each period, hence the total discounted profit is $(1+\delta) t / 2$. With BBPD, each firm earns profit $(3+\delta) t / 6$ in $\tau=1$ and $5 t / 18$ in $\tau=2$. Thus the total discounted profit is $(9+8 \delta) t / 18<(1+\delta) t / 2$. This is consistent with the general thrust of the literature that shows BBPD lowers firm profitability unless there are sufficient asymmetries.

\subsection{Location constraint}

In our model, we assumed that location choice is restricted to $[0,1]$. We now consider the case where firms may locate outside $[0,1]$. The main purpose of this exercise is to see whether location choice continues to be identical to what is obtained in the absence

\footnotetext{
${ }^{5}$ Since firm $A$ serves customers in $[0,1 / 3] \cup[1 / 2,2 / 3]$ and firm $B$ serves the rest, the average distance traveled is $\int_{0}^{1 / 3} x d x+\int_{1 / 3}^{1 / 2}(1-x) d x+\int_{1 / 2}^{2 / 3} x d x+\int_{2 / 3}^{1}(1-x) d x=11 / 36$.
} 
of dynamic consideration. ${ }^{6}$ When adapted to our model, one can verify that the static location equilibrium without location constraint in Tabuchi and Thisse (1995) is given by $a=-1 / 4, b=5 / 4$.

As in Proposition 2, we simplify analysis by assuming consumers are myopic. We show that firms' discount factor $\left(\delta_{f}=\delta\right)$ matters in that the static outcome in Tabuchi and Thisse (1995) obtains only when firms are also myopic $(\delta=0)$. Otherwise, firms choose locations closer to each other with location choice converging to that in Tabuchi and Thisse (1995) as $\delta$ decreases to 0 . This suggests that, given location constraint and quadratic transportation costs, the incentives to soften competition dominate the dynamic consideration. When the location constraint is relaxed, however, the dynamic consideration has bite, as we formalize below.

Proposition 3. Suppose $\delta_{c}=0$ and that firms can choose locations outside $[0,1]$. Then for all $\delta_{f}=\delta \in[0,1]$, the game has a unique equilibrium with locations given by

$$
a=-\frac{81-99 \delta+20 \delta^{2}}{12\left(27+9 \delta-20 \delta^{2}\right)}, \quad b=1-a .
$$

As $\delta$ decreases to 0 , a decreases monotonically and the location equilibrium converges to the static equilibrium in Tabuchi and Thisse (1995): $a=-1 / 4, b=5 / 4$.

Proof. See the technical appendix.

\section{Conclusion}

We have studied spatial competition in a model of behavior-based price discrimination in Fudenberg and Tirole (2000). We find that the static Hotelling outcome of maximal differentiation continues to emerge in equilibrium when the second-period competition is in third-degree price discrimination. Thus endogenous product choice does not intensify competition further compared to the case where product choice is fixed exogenously. The result is driven primarily by third-degree price discrimination, which no longer holds when firms can avail themselves of more sophisticated pricing tools such as personalized pricing, as shown in Choe et al. (2018). Thus our result identifies an important interplay between the type of price competition and product choice.

\section{References}

Acquisti, A. and H. R. Varian (2005), Conditioning prices on purchase history. Marketing Science, 24(3): 367-381.

\footnotetext{
${ }^{6}$ For studies of Hotelling model without the location constraint, see, for example, Tabuchi and Thisse (1995), Matsumura and Matsushima (2012) and Li and Shuai (2018).
} 
Chen, Y. (1997), Paying customers to switch. Journal of Economics and Management Strategy, 6(4): 877-897.

Chen, Y. (2005), Oligopoly price discrimination by purchase history. Chapter 4 in Pros and Cons of Price Discrimination, Swedish Competition Authority, Stockholm.

Chen, Y. and Z. J. Zhang (2009), Dynamic targeted pricing with strategic consumers. International Journal of Industrial Organization, 27(1): 43-50.

Choe, C., S. King and N. Matsushima (2018), Pricing with cookies: behavior-based price discrimination and spatial competition. Management Science, 64(12): 5669-5687.

Esteves, R.-B. (2010), Pricing with customer recognition. International Journal of Industrial Organization, 28(6): 669-681.

Ezrachi A. and M. E. Stucke (2016), Virtual Competition, Harvard University Press, Cambridge.

Fudenberg, D. and J. Tirole (2000), Customer poaching and brand switching. RAND Journal of Economics, 31(4): 634-657.

Fudenberg, D. and J. M. Villas-Boas (2006), Behavior-based price discrimination and customer recognition. Chapter 7 in Economics and Information Systems, Vol. 1, ed. by Hendershott, T., North-Holland: Amsterdam.

Fudenberg, D. and J. M. Villas-Boas (2012), Price discrimination in the digital economy. Chapter 10 in Oxford Handbook of the Digital Economy, ed. by Peitz, M. and J. Waldfogel, Oxford University Press, New York.

Jing, B. (2017), Behavior-based pricing, production efficiency, and quality differentiation. Management Science, 63(7): 2365-2376.

Li, K. J. and S. Jain (2016), Behavior-based pricing: an analysis of the impact of peerinduced fairness. Management Science, 62(9): 2705-2721.

Li, Y. and J. Shuai (2018), A welfare analysis of location space constraints with vertically separated sellers. Review of Industrial Organization, 52(1): 161-177.

Matsumura, T. and N. Matsushima (2012), Locating outside a linear city can benefit consumers. Journal of Regional Science, 52(3): 420-432.

Pazgal, A. and D. Soberman (2008), Behavior-based discrimination: Is it a winning play, and if so, when? Marketing Science, 27(6): 977-994. 
Rhee K.-E. and R. Thomadsen (2017), Behavior-based pricing in vertically differentiated industries. Management Science, 63(8): 2729-2740.

Shin, J. and K. Sudhir (2010), A customer management dilemma: when is it profitable to reward one's own customers? Marketing Science, 29(4): 671-689.

Tabuchi, T. and J.-F. Thisse (1995), Asymmetric equilibria in spatial competition. International Journal of Industrial Organization, 13, 213-227.

Villas-Boas, J. M. (1999), Dynamic competition with customer recognition. RAND Journal of Economics, 30(4): 604-631.

Zhang, J. (2011), The perils of behavior-based personalization. Marketing Science, 30(1): $170-186$.

\section{Appendix}

Proof of Proposition 1 (Sketch). Recall that there are three possible outcomes in $\tau=2:$ (1) $z=z_{A}$, (2) $z_{A}<z<z_{B}$, (3) $z_{B}=z$. We use subscript $k=1,2,3$ to denote each of these outcomes. For each $k$, denote the location of $\tau=1$ marginal consumer by $z_{k}$ and each firm's total discounted profit by $\Pi_{i k}, i=A, B$.

First, consider the $\tau=1$ pricing game given $(a, b)$. For each $k$, firms simultaneously choose $p_{A}, p_{B}$ to maximize $\Pi_{A k}, \Pi_{B k}$, leading to reaction functions $p_{A k}\left(p_{B} ; a, b\right)$ and $p_{B k}\left(p_{A} ; a, b\right)$. These reaction functions represent locally optimal prices given $k$. For firm $i$, the 'true' reaction function is derived from comparing $\Pi_{i k}, k=1,2,3$ to find $p_{i k}$ that leads to a global optimum. We then solve the two true reaction functions simultaneously for equilibrium prices $p_{A}^{*}(a, b), p_{B}^{*}(a, b)$, and the marginal consumer's location $z^{*}(a, b)$. In the technical appendix, we show that the solution exists only when $k=2$ with sufficient conditions as given in Proposition 1 ; in other cases $k=1,3$, the two reaction functions do not intersect.

Next, consider the $\tau=1$ location game. Substitute $p_{A}^{*}(a, b), p_{B}^{*}(a, b)$, and $z^{*}(a, b)$ into each firm's profit function and denote them by $\Pi_{A 2}^{*}(a, b), \Pi_{B 2}^{*}(a, b)$ where the second subscript indicates that the pricing equilibrium is possible only when $k=2$. Differentiating these profit functions, one can show $\partial \Pi_{A 2}^{*} / \partial a<0$ for all $a, b \in[0,1]$ and $\partial \Pi_{B 2}^{*} / \partial b>0$ for all $a, b \in[0,1]$. Thus $a=0, b=1$ is a candidate equilibrium, which leads to the two-way poaching outcome in $\tau=2$. Substituting $a=0, b=1$ into $p_{A}^{*}(a, b), p_{B}^{*}(a, b)$, (1), (2), (3), and (4) gives us the equilibrium stated in the proposition. 


\section{Technical Appendix}

In this appendix, we provide the detailed proof of the results in our paper. We have used Mathematica for the calculations and figures included in this appendix.

\section{Proof of Proposition 1}

\section{Second period}

We start with the second-period prices.

We define four prices:

$p_{A o}$ is the price of Firm A for its old customers,

$p_{A n}$ is the price of Firm A for its new customers,

$p_{B o}$ is the price of Firm B for its old customers,

$p_{B n}$ is the price of Firm B for its new customers.

The location of the indifferent consumer in Firm A's turf, $z_{A}$, is derived by solving the following equation with respect to $z_{A}$ :

Solve $\left[-p_{A O}-t\left(z_{A}-a\right)^{2}=-p_{B n}-t\left(z_{A}-b\right)^{2}, z_{A}\right]$

$\left\{\left\{z_{A} \rightarrow \frac{a^{2} t-b^{2} t+p_{A o}-p_{B n}}{2(a-b) t}\right\}\right\}$

We set the location of the indifferent consumer in Firm A's turf, $z_{A}$ :

$z_{A}=\frac{a^{2} t-b^{2} t+p_{A O}-p_{B n}}{2(a-b) t}$

$\frac{a^{2} t-b^{2} t+p_{A 0}-p_{B n}}{2(a-b) t}$

The location of the indifferent consumer in Firm B's turf, $z_{B}$, is derived by solving the following equation with respect to $z_{B}$ :

Solve $\left[-p_{B O}-t\left(z_{B}-b\right)^{2}=-p_{A n}-t\left(z_{B}-a\right)^{2}, z_{B}\right]$

$\left\{\left\{z_{B} \rightarrow \frac{a^{2} t-b^{2} t+p_{A n}-p_{B O}}{2(a-b) t}\right\}\right\}$

We set the location of the indifferent consumer in Firm B's turf, $z_{B}$ :

$z_{B}=\frac{a^{2} t-b^{2} t+p_{A n}-p_{B O}}{2(a-b) t}$

$\frac{a^{2} t-b^{2} t+p_{A n}-p_{B O}}{2(a-b) t}$

The first-order differentials of Firm A's profit with respect to $p_{A o}$ and $p_{A n}$ are

Factor $\left[D\left[p_{A 0} z_{A}+p_{A n}\left(z_{B}-z\right), p_{A 0}\right]\right]$

$\frac{a^{2} t-b^{2} t+2 p_{A o}-p_{B n}}{2(a-b) t}$

Factor $\left[D\left[p_{A O} z_{A}+p_{A n}\left(z_{B}-z\right), p_{A n}\right]\right]$

$-\frac{-a^{2} t+b^{2} t+2 a t z-2 b t z-2 p_{A n}+p_{B O}}{2(a-b) t}$

Similarly, the first-order differentials of Firm B's profit with respect to $p_{B o}$ and $p_{B n}$ are 


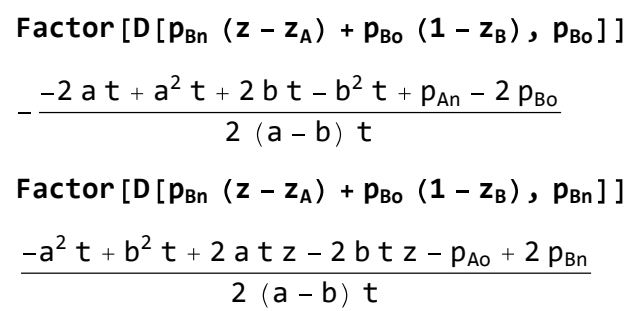

The four first-order differentials give us the following simultaneous equations, which we solve for the second-period prices.

$$
\begin{aligned}
& \text { Simplify }\left[\text { Solve } \left[\left\{\frac{a^{2} t-b^{2} t+2 p_{A o}-p_{B n}}{2(a-b) t}=0,\right.\right.\right. \\
& -\frac{-a^{2} t+b^{2} t+2 a t z-2 b t z-2 p_{A n}+p_{B o}}{2(a-b) t}=0,-\frac{-2 a t+a^{2} t+2 b t-b^{2} t+p_{A n}-2 p_{B o}}{2(a-b) t}=0, \\
& \left.\left.\left.\quad \frac{-a^{2} t+b^{2} t+2 a t z-2 b t z-p_{A o}+2 p_{B n}}{2(a-b) t}=0\right\},\left\{p_{A o}, p_{A n}, p_{B n}, p_{B o}\right\}\right]\right] \\
& \left\{\left\{p_{A o} \rightarrow-\frac{1}{3}(a-b) t(a+b+2 z), p_{A n} \rightarrow-\frac{1}{3}(a-b) t(2+a+b-4 z),\right.\right. \\
& \left.\left.p_{B n} \rightarrow \frac{1}{3}(a-b) t(a+b-4 z), p_{B o} \rightarrow \frac{1}{3}(a-b) t(-4+a+b+2 z)\right\}\right\}
\end{aligned}
$$

Substituting the above prices into $z_{A}$, we have the equilibrium $z_{A}$ in period 2:

$$
\begin{aligned}
\text { Factor } & {\left[z_{A} / \cdot\left\{p_{A o} \rightarrow-\frac{1}{3}(a-b) t(a+b+2 z), p_{A n} \rightarrow-\frac{1}{3}(a-b) t(2+a+b-4 z),\right.\right.} \\
p_{B n} & \left.\left.\rightarrow \frac{1}{3}(a-b) t(a+b-4 z), p_{B o} \rightarrow \frac{1}{3}(a-b) t(-4+a+b+2 z)\right\}\right] \\
\frac{1}{6}(a+b+2 z) &
\end{aligned}
$$

This $z_{A}$ is in the range [0, $\mathrm{z}$ ] if and only if $\mathrm{z} \geq(\mathrm{a}+\mathrm{b}) / 4$. If $\mathrm{z} \leq(\mathrm{a}+\mathrm{b}) / 4$, we need to consider a corner solution $\left(z_{A}=\mathrm{z}\right)$, which is discussed later.

Similarly, substituting the above prices into $z_{B}$, we have the equilibrium $z_{B}$ in period 2 :

$$
\begin{aligned}
& \text { Factor }\left[z_{B} / \cdot\left\{p_{A o} \rightarrow-\frac{1}{3}(a-b) t(a+b+2 z), p_{A n} \rightarrow-\frac{1}{3}(a-b) t(2+a+b-4 z),\right.\right. \\
& \left.\left.\quad p_{B n} \rightarrow \frac{1}{3}(a-b) t(a+b-4 z), p_{B o} \rightarrow \frac{1}{3}(a-b) t(-4+a+b+2 z)\right\}\right] \\
& \frac{1}{6}(2+a+b+2 z)
\end{aligned}
$$

This $z_{B}$ is in the range $[z, 1]$ if and only if $z \leq(2+a+b) / 4$. If $z \geq(2+a+b) / 4$, we need to consider a corner solution $\left(z_{B}=\mathrm{z}\right)$, which is discussed later.

Based on the above discussions, we have three cases: (i) $0 \leq z \leq(a+b) / 4$, (ii) $(\mathrm{a}+\mathrm{b}) / 4<\mathrm{z}<(2+\mathrm{a}+\mathrm{b}) / 4$, (iii) $(2+\mathrm{a}+\mathrm{b}) / 4 \leq \mathrm{z} \leq 1$.

(Case i) $0 \leq \mathrm{Z} \leq(\mathrm{a}+\mathrm{b}) / 4$.

In this case, we have $z_{A}=z$, hence Firm B cannot poach any customer in Firm A's turf. As a result, $p_{B n}=0$. Anticipating this, Firm A sets the highest $p_{A o}$ that leads to $z_{A}=z \cdot z_{A}$, just equals to $z$. This is found below.

Solve $\left[\left\{z_{A}=z, p_{B n}=0\right\},\left\{p_{A 0}, p_{B n}\right\}\right]$

$\left\{\left\{p_{A O} \rightarrow-(a-b) t(a+b-2 z), p_{B n} \rightarrow \theta\right\}\right\}$ 
For the optimal pricing in Firm B's turf, we can use the first-order differentials we have already derived:

The first - order differential of Firm $A\left(p_{A n}\right):-\frac{-a^{2} t+b^{2} t+2 a t z-2 b t z-2 p_{A n}+p_{B O}}{2(a-b) t}$
The first - order differential of Firm $B\left(p_{B O}\right):-\frac{-2 a t+a^{2} t+2 b t-b^{2} t+p_{A n}-2 p_{B O}}{2(a-b) t}$

This leads to the following second-period prices:

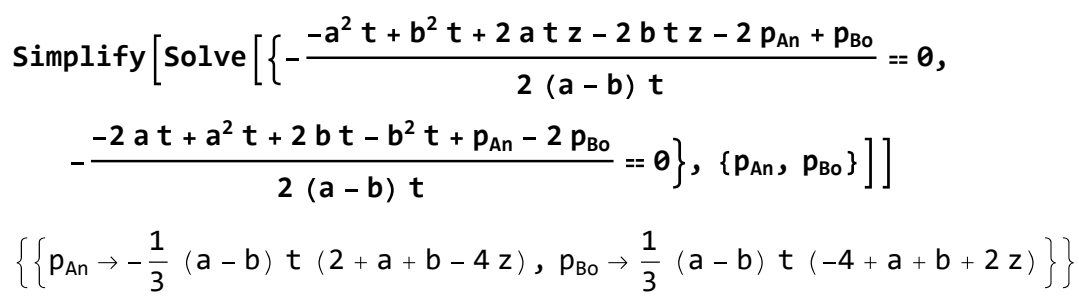

Substituting the above prices into $z_{B}$, we have the equilibrium $z_{B}$ in period 2 when $0 \leq \mathrm{z} \leq(\mathrm{a}+\mathrm{b}) / 4$ :

Factor $\left[z_{B} / \cdot\left\{p_{A n} \rightarrow-\frac{1}{3}(a-b) t(2+a+b-4 z), p_{B o} \rightarrow \frac{1}{3}(a-b) t(-4+a+b+2 z)\right\}\right]$

$\frac{1}{6}(2+a+b+2 z)$

Using the above outcomes, we derive the second period profit of Firm A in case (i) given the first-period z:

$$
\begin{aligned}
& \text { Factor }\left[p_{A o} z_{A}+p_{A n}\left(z_{B}-z\right) / \cdot\left\{p_{A o} \rightarrow-(a-b) t(a+b-2 z),\right.\right. \\
& \left.\left.\quad p_{A n} \rightarrow-\frac{1}{3}(a-b) t(2+a+b-4 z), p_{B o} \rightarrow \frac{1}{3}(a-b) t(-4+a+b+2 z), p_{B n} \rightarrow \theta\right\}\right] \\
& -\frac{1}{18}(a-b) t\left(4+4 a+a^{2}+4 b+2 a b+b^{2}-16 z+10 a z+10 b z-20 z^{2}\right)
\end{aligned}
$$

Similarly, using the above outcomes, we derive the second period profit of Firm B in case (i) given the first-period z:s

$$
\begin{aligned}
& \text { Factor }\left[p_{B n}\left(z-z_{A}\right)+p_{B o}\left(1-z_{B}\right) / .\left\{p_{A o} \rightarrow-(a-b) t(a+b-2 z),\right.\right. \\
& \left.\left.\quad p_{A n} \rightarrow-\frac{1}{3}(a-b) t(2+a+b-4 z), p_{B o} \rightarrow \frac{1}{3}(a-b) t(-4+a+b+2 z), p_{B n} \rightarrow \theta\right\}\right] \\
& -\frac{1}{18}(a-b) t(-4+a+b+2 z)^{2}
\end{aligned}
$$

(Case ii) $(\mathrm{a}+\mathrm{b}) / 4<\mathrm{z}<(2+\mathrm{a}+\mathrm{b}) / 4$.

In this case, we have an interior solution with two-way poaching. Therefore, we can use the second-period prices we have already obtained previously, reproduced below:

$$
\begin{gathered}
\left\{p_{A o} \rightarrow-\frac{1}{3}(a-b) t(a+b+2 z), p_{A n} \rightarrow-\frac{1}{3}(a-b) t(2+a+b-4 z),\right. \\
\left.p_{B n} \rightarrow \frac{1}{3}(a-b) t(a+b-4 z), p_{B o} \rightarrow \frac{1}{3}(a-b) t(-4+a+b+2 z)\right\}
\end{gathered}
$$

Thus firm A's second-period profit given $z$ can be derived as 


$$
\begin{aligned}
& \text { Factor }\left[p_{A o} z_{A}+p_{A n}\left(z_{B}-z\right) / \cdot\left\{p_{A o} \rightarrow-\frac{1}{3}(a-b) t(a+b+2 z), p_{A n} \rightarrow-\frac{1}{3}(a-b) t(2+a+b-4 z),\right.\right. \\
& \left.\left.\quad p_{B n} \rightarrow \frac{1}{3}(a-b) t(a+b-4 z), p_{B o} \rightarrow \frac{1}{3}(a-b) t(-4+a+b+2 z)\right\}\right] \\
& -\frac{1}{9}(a-b) t\left(2+2 a+a^{2}+2 b+2 a b+b^{2}-8 z-2 a z-2 b z+10 z^{2}\right)
\end{aligned}
$$

Similarly, firm B's profit given $\mathrm{z}$ is

$$
\begin{aligned}
& \text { Factor }\left[p_{B n}\left(z-z_{A}\right)+p_{B o}\left(1-z_{B}\right) / \cdot\left\{p_{A o} \rightarrow-\frac{1}{3}(a-b) t(a+b+2 z),\right.\right. \\
& \left.\left.\quad p_{A n} \rightarrow-\frac{1}{3}(a-b) t(2+a+b-4 z), p_{B n} \rightarrow \frac{1}{3}(a-b) t(a+b-4 z), p_{B o} \rightarrow \frac{1}{3}(a-b) t(-4+a+b+2 z)\right\}\right] \\
& -\frac{1}{9}(a-b) t\left(8-4 a+a^{2}-4 b+2 a b+b^{2}-8 z-2 a z-2 b z+10 z^{2}\right)
\end{aligned}
$$

(Case iii) $(2+\mathrm{a}+\mathrm{b}) / 4 \leq \mathrm{z} \leq 1$.

In this case, $z_{B}=z$, hence Firm A cannot poach any customer in Firm B's turf. As a result, $p_{A n}=0$. Given $p_{A n}=0$, Firm B chooses the highest $p_{B o}$ that leads to $z_{B}=z$ :

Solve $\left[\left\{z_{B}=z, p_{A n}=\theta\right\},\left\{p_{B o}, p_{A n}\right\}\right]$

$\left\{\left\{p_{B O} \rightarrow(a-b) t(a+b-2 z), p_{A n} \rightarrow 0\right\}\right\}$

For the optimal pricing in Firm A's turf, we can use the first-order differentials we have already derived:

The first - order differential of Firm $A\left(p_{A 0}\right): \frac{a^{2} t-b^{2} t+2 p_{A O}-p_{B n}}{2(a-b) t}$

The first - order differential of Firm $B\left(p_{B n}\right): \frac{-a^{2} t+b^{2} t+2 a t z-2 b t z-p_{A O}+2 p_{B n}}{2(a-b) t}$

Solving the following simultaneous equations gives us the second-period prices.

$$
\begin{aligned}
& \text { Simplify }\left[\text { Solve }\left[\left\{\frac{a^{2} t-b^{2} t+2 p_{A o}-p_{B n}}{2(a-b) t}=0, \frac{-a^{2} t+b^{2} t+2 a t z-2 b t z-p_{A o}+2 p_{B n}}{2(a-b) t}=0\right\},\left\{p_{A o}, p_{B n}\right\}\right]\right] \\
& \left\{\left\{p_{A o} \rightarrow-\frac{1}{3}(a-b) t(a+b+2 z), p_{B n} \rightarrow \frac{1}{3}(a-b) t(a+b-4 z)\right\}\right\}
\end{aligned}
$$

Substituting the second-period prices into $z_{A}$, we have the equilibrium $z_{A}$ in period 2 when $\mathrm{z} \geq$ $(2+a+b) / 4$ :

$$
\begin{aligned}
& \text { Factor }\left[z_{A} /\right. \\
& \left.\qquad\left\{p_{A O} \rightarrow-\frac{1}{3}(a-b) t(a+b+2 z), p_{A n} \rightarrow 0, p_{B O} \rightarrow(a-b) t(a+b-2 z), p_{B n} \rightarrow \frac{1}{3}(a-b) t(a+b-4 z)\right\}\right] \\
& \frac{1}{6}(a+b+2 z)
\end{aligned}
$$

Using the above prices, $z_{A}$ and $z_{B}=z$, we derive firm A's second-period profit given $z$ as follows:

$$
\begin{aligned}
& \text { Factor }\left[p_{A o} z_{A}+p_{A n}\left(z_{B}-z\right) /\right. \\
& \left.\qquad\left\{p_{A o} \rightarrow-\frac{1}{3}(a-b) t(a+b+2 z), p_{A n} \rightarrow \theta, p_{B o} \rightarrow(a-b) t(a+b-2 z), p_{B n} \rightarrow \frac{1}{3}(a-b) t(a+b-4 z)\right\}\right] \\
& -\frac{1}{18}(a-b) t(a+b+2 z)^{2}
\end{aligned}
$$

Similarly, firm B's second-period profit given $\mathrm{z}$ is 


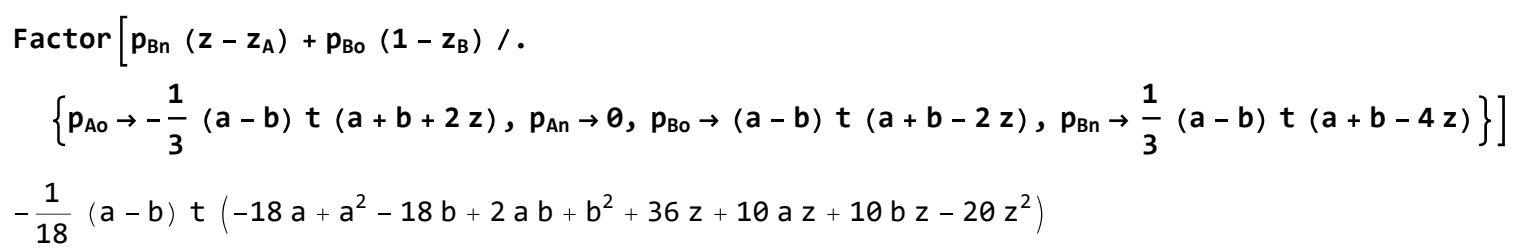

\section{First period - Prices}

In our calculation, we denote firms' discount factor by $\delta_{f}$ and consumers' discount factor by $\delta_{c}$. In Proposition 1, we focus on the case, $\delta_{f}=\delta_{f}=\delta$. In Propositions 2 and 3, we focus on the case, $\delta_{f}=\delta$ and $\delta_{c}=0$.

We need to consider three cases: (i) $0 \leq \mathrm{z} \leq(\mathrm{a}+\mathrm{b}) / 4$, (ii) $(\mathrm{a}+\mathrm{b}) / 4<\mathrm{z}<(2+\mathrm{a}+\mathrm{b}) / 4$, (iii) $(2+\mathrm{a}+\mathrm{b}) / 4 \leq$ $\mathrm{z} \leq 1$.

(Case i) $0 \leq z \leq(a+b) / 4$.

From the previous analysis, we have the second-period prices given as follow.

$$
\begin{aligned}
\left\{p_{A 0}\right. & \rightarrow-(a-b) t(a+b-2 z), p_{A n} \rightarrow-\frac{1}{3}(a-b) t(2+a+b-4 z), \\
p_{B o} & \left.\rightarrow \frac{1}{3}(a-b) t(-4+a+b+2 z), p_{B n} \rightarrow \theta\right\}
\end{aligned}
$$

Anticipating the second period prices, consumers choose one of the first-period prices $p_{A}$ or $p_{B}$ ( $p_{A}$ is the first-period price of firm $\mathrm{A}$ and $p_{B}$ is the first-period price of firm $\mathrm{B}$ )

The location of the indifferent consumer, $z$, is derived from the following equation.:

$$
\begin{aligned}
& \text { Solve }\left[-p_{A}-t(z-a)^{2}-\delta c\left((-(a-b) t(a+b-2 z))+t(z-a)^{2}\right)==\right. \\
& \left.\quad-p_{B}-t(z-b)^{2}-\delta c\left(\left(\frac{1}{3}(b-a) t(2+a+b-4 z)\right)+t(z-a)^{2}\right), z\right] \\
& \left\{\left\{z \rightarrow\left(-3 a^{2} t+3 b^{2} t-2 a t \delta c+2 a^{2} t \delta c+2 b t \delta c-2 b^{2} t \delta c-3 p_{A}+3 p_{B}\right) /(2(a-b) t(-3+\delta c))\right\}\right\}
\end{aligned}
$$

We set the location of the indifferent consumers $z$ :

$z=\left(-3 a^{2} t+3 b^{2} t-2 a t \delta c+2 a^{2} t \delta c+2 b t \delta c-2 b^{2} t \delta c-3 p_{A}+3 p_{B}\right) /(2(a-b) t(-3+\delta c))$

$\frac{-3 a^{2} t+3 b^{2} t-2 a t \delta c+2 a^{2} t \delta c+2 b t \delta c-2 b^{2} t \delta c-3 p_{A}+3 p_{B}}{2(a-b) t(-3+\delta c)}$

Next, we derive the condition for $z$ to be in the range $[0,(a+b) / 4]$ by solving the following equations.

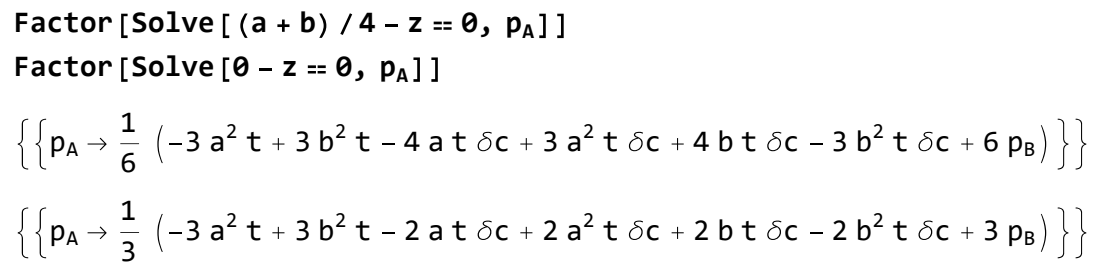

Simplifying the above, the condition can be stated as follows.

$p_{B}+\frac{(b-a) t((a+b)(3-2 \delta c)+2 \delta c)}{3} \geq p_{A} \geq p_{B}+\frac{(b-a) t(3(a+b)(1-\delta c)+4 \delta c)}{6}$

Note that if $p_{A}$ is larger than $\mathrm{p}_{\mathrm{B}}+\frac{(\mathrm{b}-\mathrm{a}) \mathrm{t}((\mathrm{a}+\mathrm{b})(3-2 \delta \mathrm{c})+2 \delta \mathrm{c})}{3}, z$ becomes zero. 
Next, we solve for the pricing equilibrium in the first period.

From the previous analysis, we have each firm's second-period profit given as follows.

$\pi_{A 2}:-\frac{1}{18}(a-b) t\left(4+4 a+a^{2}+4 b+2 a b+b^{2}-16 z+10 a z+10 b z-20 z^{2}\right)$

$\pi_{B 2}:-\frac{1}{18}(a-b) t(-4+a+b+2 z)^{2}$

First, the derivative of firm A's total discounted profit with respect to $p_{A}$ is

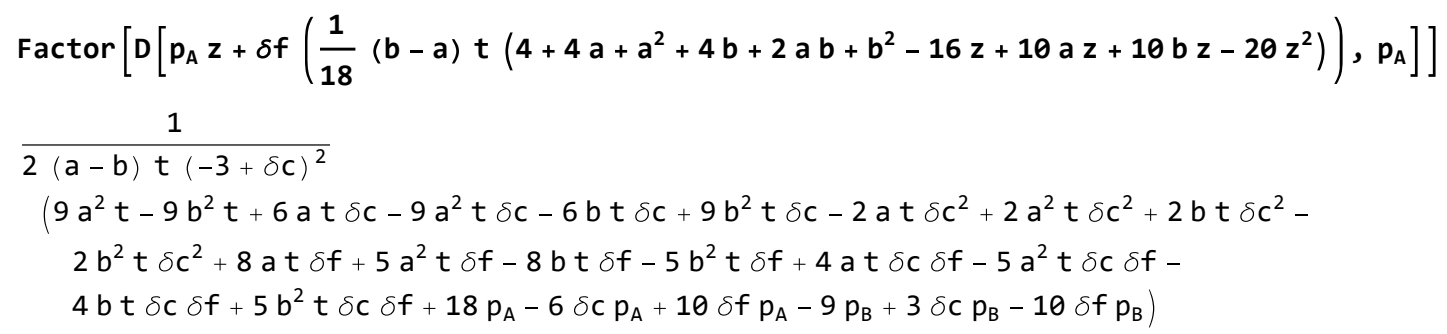

Using the derivative, we obtain the reaction function of Firm $A$ in the range, $0 \leq z \leq(a+b) / 4$ :

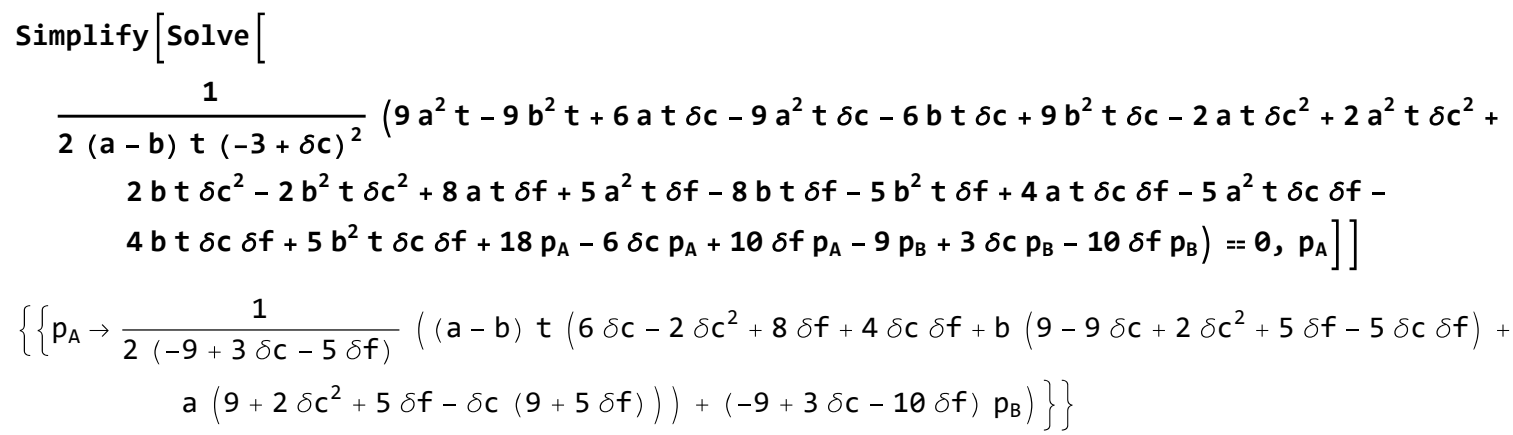

Since the above reaction function may prescribe $z$ outside the required range, we need the condition that indeed guarantees, $0 \leq \mathrm{Z} \leq(\mathrm{a}+\mathrm{b}) / 4$.

We have already obtained the condition that $\mathrm{z}$ is between 0 and $(\mathrm{a}+\mathrm{b}) / 4$ as follows:

$p_{B}+\frac{(b-a) t((a+b)(3-2 \delta c)+2 \delta c)}{3} \geq p_{A} \geq p_{B}+\frac{(b-a) t(3(a+b)(1-\delta c)+4 \delta c)}{6}$

If the following outcomes are positive, the reaction function satisfies the above inequalities:

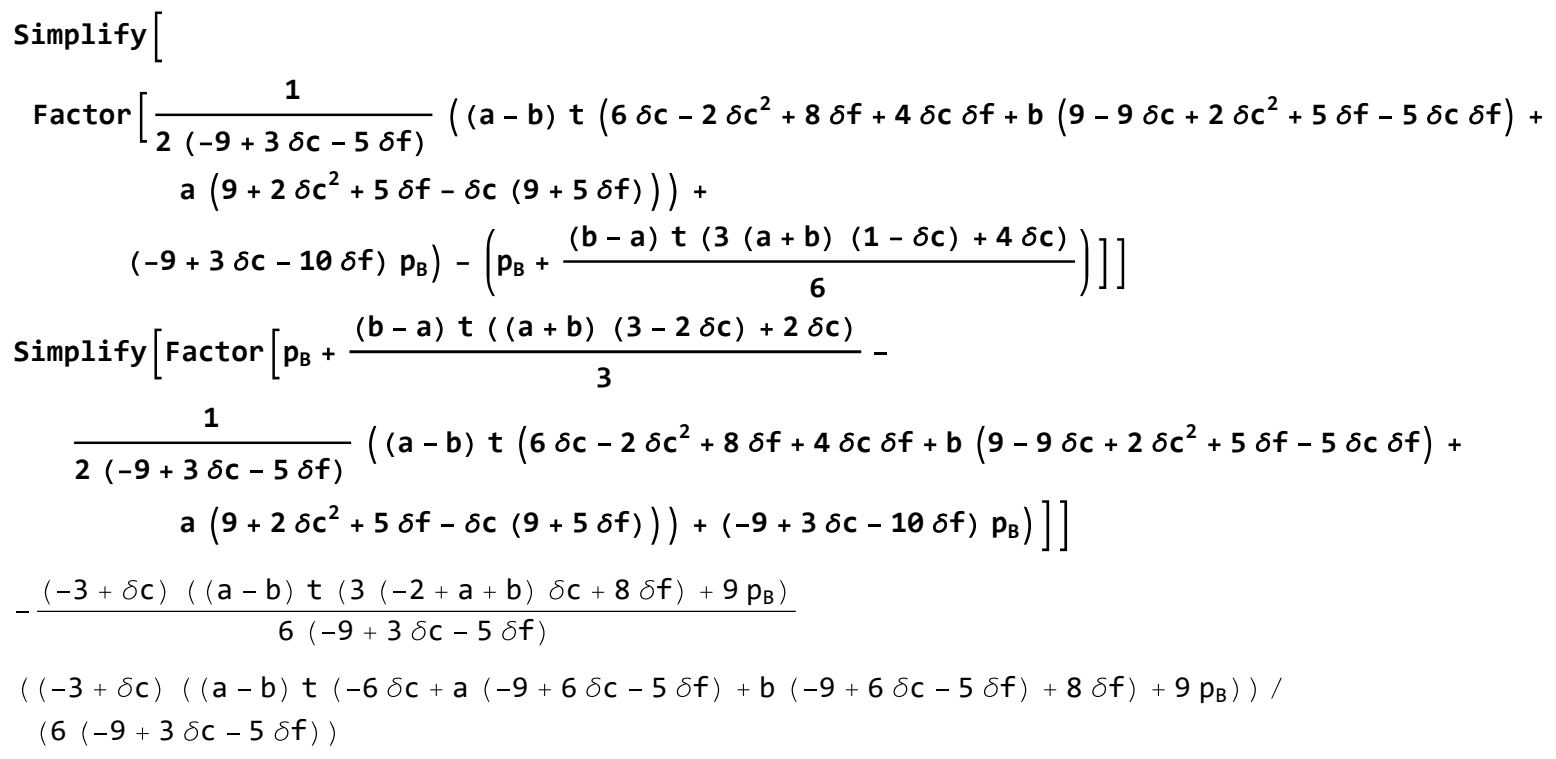

We derive the threshold values of $p_{B}$ such that each of the outcomes equals zero: 


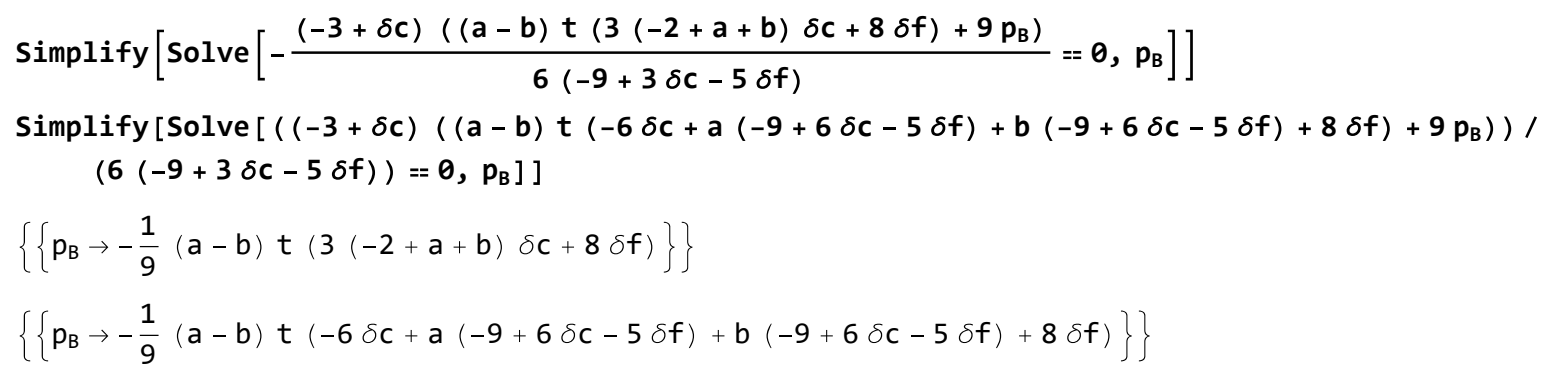

Therefore, if $p_{B}$ satisfies the following inequalities, the reaction function of Firm $\mathrm{A}$ is in the range, $0 \leq \mathrm{z} \leq(\mathrm{a}+\mathrm{b}) / 4$ :

$-\frac{1}{9}(b-a) t((a+b)(9-6 \delta c+5 \delta f)+6 \delta c-8 \delta f) \leq p_{B} \leq \frac{(b-a) t(3(-2+a+b) \delta c+8 \delta f)}{9}$

Note that if $p_{B}$ is smaller than the left-hand side value of the inequality, Firm A abandons to supply in period 1 .

Similarly, using the above outcomes, we derive the first-order derivative of Firm B's profit with respect to $p_{B}$

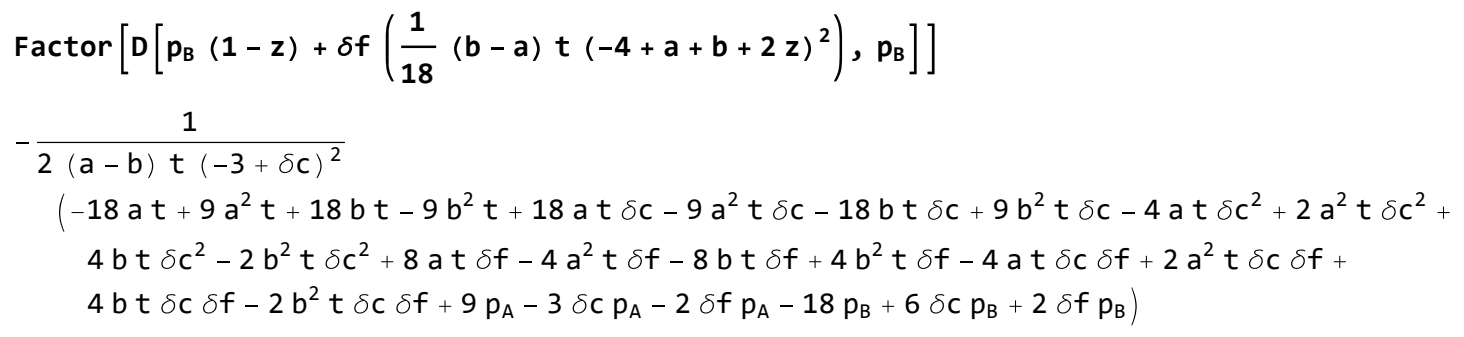

Using the derivative, we obtain the reaction function of Firm $B$ in the range, $0 \leq \mathrm{Z} \leq(\mathrm{a}+\mathrm{b}) / 4$ :

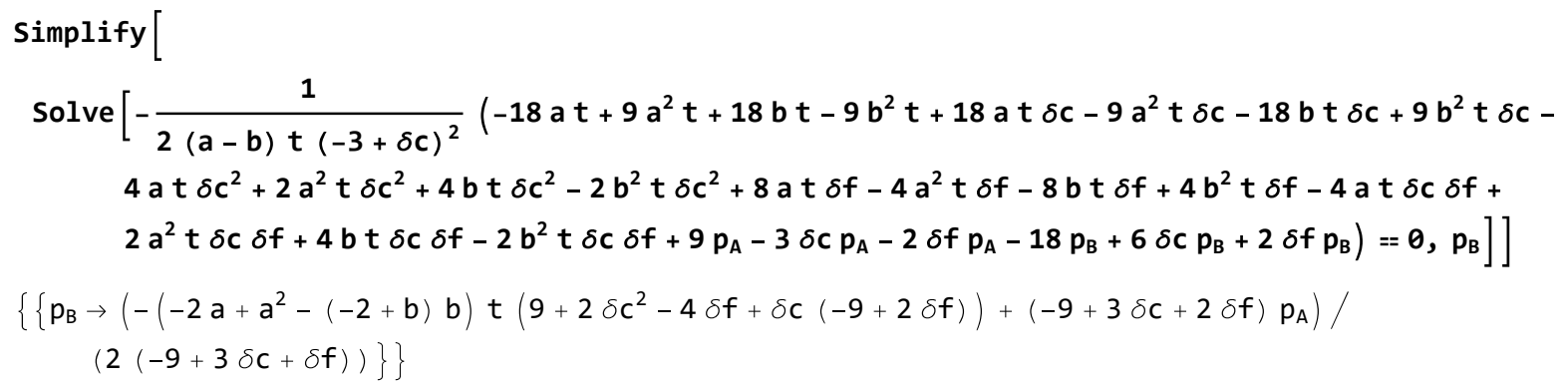

Since the above reaction function may prescribe $\mathrm{z}$ outside the required range, we need the condition that indeed guarantees $0 \leq \mathrm{z} \leq(\mathrm{a}+\mathrm{b}) / 4$.

We have already obtained the condition that $\mathrm{z}$ is between 0 and $(\mathrm{a}+\mathrm{b}) / 4$ as follows:

$p_{B}+\frac{(b-a) t((a+b)(3-2 \delta c)+2 \delta c)}{3} \geq p_{A} \geq p_{B}+\frac{(b-a) t(3(a+b)(1-\delta c)+4 \delta c)}{6}$.

If $p_{A}$ is larger than $\mathrm{p}_{\mathrm{B}}+\frac{(\mathrm{b}-\mathrm{a}) \mathrm{t}((\mathrm{a}+\mathrm{b})(3-2 \delta \mathrm{c})+2 \delta \mathrm{c})}{3}, z$ becomes zero. In this case, Firm $\mathrm{B}$ chooses the following $p_{B}$ which just leads to $\mathrm{z}=0$.

$\mathrm{p}_{B} \rightarrow \mathrm{p}_{\mathrm{A}}-\frac{(\mathrm{b}-\mathrm{a}) \mathrm{t}((\mathrm{a}+\mathrm{b})(3-2 \delta \mathrm{c})+2 \delta \mathrm{c})}{3}$.

If the following outcomes are positive, the reaction function satisfies the above inequalities, $0 \leq \mathrm{z}$ $\leq(\mathrm{a}+\mathrm{b}) / 4$ : 


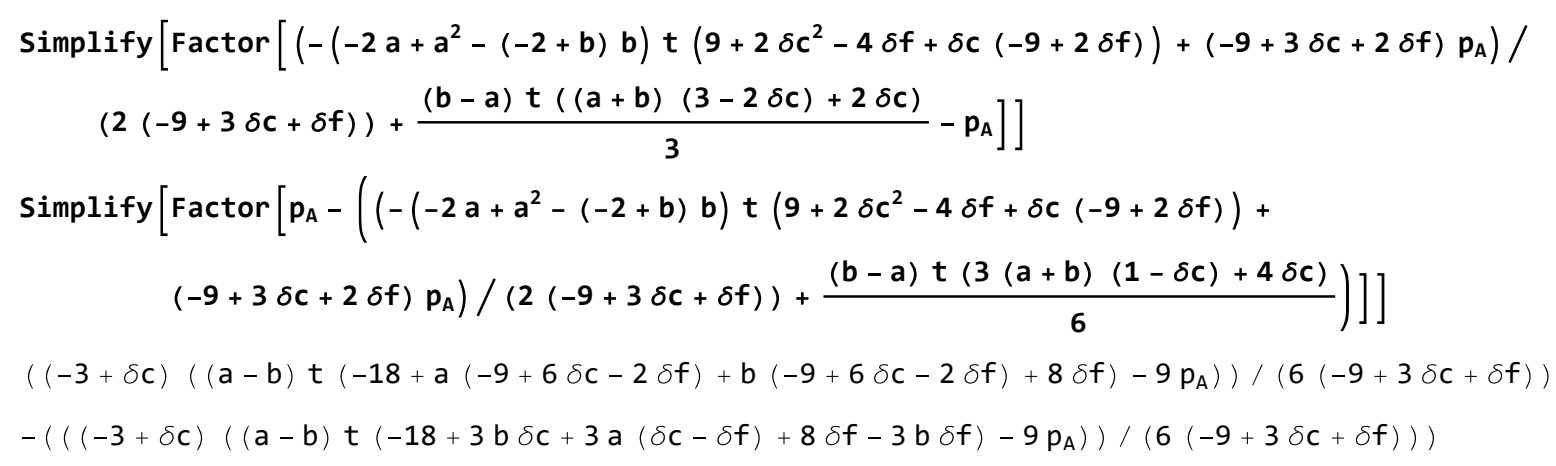

We derive the threshold values of $p_{A}$ such that each of the outcomes equals zero:

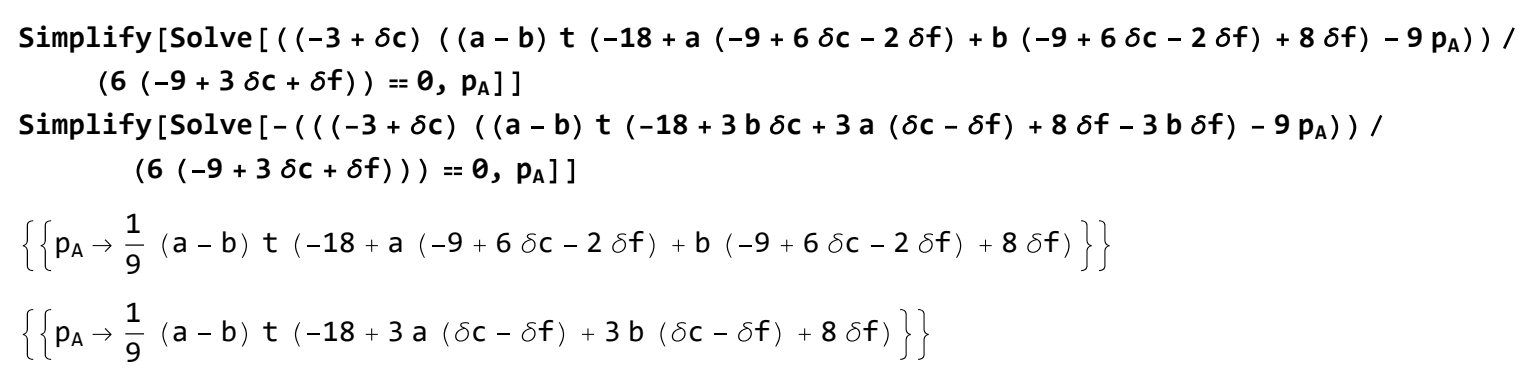

Therefore, if $p_{A}$ satisfies the following inequalities, the reaction function of Firm $\mathrm{B}$ is in the range, $0 \leq \mathrm{z} \leq(\mathrm{a}+\mathrm{b}) / 4$ :

$\frac{(b-a) t(18+(a+b)(9-6 \delta c+2 \delta f)-8 \delta f)}{9} \geq p_{A} \geq \frac{(b-a) t(18-3(a+b)(\delta c-\delta f)-8 \delta f)}{9}$

Note that if $p_{A}$ is larger than the left-hand side value of the inequality, Firm B chooses the following $p_{B}$, which leads to $\mathrm{z}=0$.

$p_{B} \rightarrow p_{A}-\frac{(b-a) t((a+b)(3-2 \delta c)+2 \delta c)}{3}$

(Case ii) $(\mathrm{a}+\mathrm{b}) / 4<\mathrm{z}<(2+\mathrm{a}+\mathrm{b}) / 4$

From the previous analysis, we have the second-period prices given as follow.

$$
\begin{gathered}
\left\{p_{A o} \rightarrow-\frac{1}{3}(a-b) t(a+b+2 z), p_{A n} \rightarrow-\frac{1}{3}(a-b) t(2+a+b-4 z),\right. \\
\left.p_{B n} \rightarrow \frac{1}{3}(a-b) t(a+b-4 z), p_{B o} \rightarrow \frac{1}{3}(a-b) t(-4+a+b+2 z)\right\}
\end{gathered}
$$

Anticipating the second period prices, consumers choose one of the first-period prices $p_{A}$ or $p_{B}$ ( $p_{A}$ is the first-period price of firm $\mathrm{A}$ and $p_{B}$ is the first-period price of firm $\mathrm{B}$ )

The location of the indifferent consumer, $z$, is derived by the following equation:

$$
\begin{aligned}
& \text { Solve }\left[-p_{A}-t(z-a)^{2}-\delta c\left(\frac{1}{3}(a-b) t(a+b-4 z)+t(z-b)^{2}\right)=\right. \\
& \left.-p_{B}-t(z-b)^{2}-\delta c\left(\left(\frac{1}{3}(b-a) t(2+a+b-4 z)\right)+t(z-a)^{2}\right), z\right] \\
& \left\{\left\{z \rightarrow \frac{3 a^{2} t-3 b^{2} t+2 a t \delta c-a^{2} t \delta c-2 b t \delta c+b^{2} t \delta c+3 p_{A}-3 p_{B}}{2(a-b) t(3+\delta c)}\right\}\right\}
\end{aligned}
$$

We set the location of the indifferent consumers $\mathrm{z}$ : 


$$
\begin{aligned}
& z=\frac{3\left(p_{B}-p_{A}\right)}{2(b-a) t(3+\delta c)}+\frac{((a+b)(3-\delta c)+2 \delta c)}{2(3+\delta c)} \\
& \frac{(a+b)(3-\delta c)+2 \delta c}{2(3+\delta c)}+\frac{3\left(-p_{A}+p_{B}\right)}{2(-a+b) t(3+\delta c)}
\end{aligned}
$$

We derive the condition that the location of the indifferent consumers, $z$, is between $(a+b) / 4$ and $(2+a+b) / 4$, by solving the following simultaneous equations

Simplify [Solve $\left.\left[z-(a+b) / 4=0, p_{A}\right]\right]$

Simplify [Solve $\left.\left[(2+a+b) / 4-z=0, p_{A}\right]\right]$

$$
\begin{aligned}
& \left\{\left\{p_{A} \rightarrow \frac{1}{6}\left((a-b) t(3 a(-1+\delta c)+3 b(-1+\delta c)-4 \delta c)+6 p_{B}\right)\right\}\right\} \\
& \left\{\left\{p_{A} \rightarrow \frac{1}{6}\left((a-b) t(6+3 a(-1+\delta c)+3 b(-1+\delta c)-2 \delta c)+6 p_{B}\right)\right\}\right\}
\end{aligned}
$$

By simplifying the above values of $p_{A}$, we have the condition that $z$ is between $(\mathrm{a}+\mathrm{b}) / 4$ and $(2+a+b) / 4$ as follows:

$p_{B}+\frac{(b-a) t(3(a+b)(1-\delta c)-2(3-\delta c))}{6}<p_{A}<p_{B}+\frac{(b-a) t(3(a+b)(1-\delta c)+4 \delta c)}{6}$

We have already derived the 2nd period profits of Firms A and B as follows:

$$
\begin{aligned}
& \pi_{A 2}:-\frac{1}{9}(a-b) t\left(2+2 a+a^{2}+2 b+2 a b+b^{2}-8 z-2 a z-2 b z+10 z^{2}\right) \\
& \pi_{B 2}:-\frac{1}{9}(a-b) t\left(8-4 a+a^{2}-4 b+2 a b+b^{2}-8 z-2 a z-2 b z+10 z^{2}\right)
\end{aligned}
$$

Using the above outcomes, we derive the first-order derivative of Firm A's total discounted profit with respect to $p_{A}$

$$
\begin{aligned}
& \text { Factor }\left[D\left[p_{A} z+\delta f \frac{1}{9}(b-a) t\left(2+2 a+a^{2}+2 b+2 a b+b^{2}-8 z-2 a z-2 b z+10 z^{2}\right), p_{A}\right]\right] \\
& -\frac{1}{2(a-b) t(3+\delta c)^{2}}\left(-9 a^{2} t+9 b^{2} t-6 a t \delta c+6 b t \delta c-2 a t \delta c^{2}+\right. \\
& \quad a^{2} t \delta c^{2}+2 b t \delta c^{2}-b^{2} t \delta c^{2}-8 a t \delta f+8 a^{2} t \delta f+8 b t \delta f-8 b^{2} t \delta f+4 a t \delta c \delta f- \\
& \left.\quad 4 a^{2} t \delta c \delta f-4 b t \delta c \delta f+4 b^{2} t \delta c \delta f-18 p_{A}-6 \delta c p_{A}+10 \delta f p_{A}+9 p_{B}+3 \delta c p_{B}-10 \delta f p_{B}\right)
\end{aligned}
$$

Using the derivative, we obtain the reaction function of Firm $A$ in the range, $(\mathrm{a}+\mathrm{b}) / 4<\mathrm{z}<(2+\mathrm{a}+\mathrm{b}) / 4$ :

$$
\begin{aligned}
& \text { Simplify }[\text { Solve }[ \\
& \begin{array}{r}
-\frac{1}{2(a-b) t(3+\delta c)^{2}}\left(-9 a^{2} t+9 b^{2} t-6 a t \delta c+6 b t \delta c-2 a t \delta c^{2}+a^{2} t \delta c^{2}+2 b t \delta c^{2}-b^{2} t \delta c^{2}-\right. \\
\quad 8 a t \delta f+8 a^{2} t \delta f+8 b t \delta f-8 b^{2} t \delta f+4 a t \delta c \delta f-4 a^{2} t \delta c \delta f-4 b t \delta c \delta f+
\end{array} \\
& \left.\quad \begin{array}{rl}
\left.\left.\left.4 b^{2} t \delta c \delta f-18 p_{A}-6 \delta c p_{A}+10 \delta f p_{A}+9 p_{B}+3 \delta c p_{B}-10 \delta f p_{B}\right)=0, p_{A}\right]\right] \\
\left\{\left\{p_{A} \rightarrow\right.\right. & \frac{1}{2(9+3 \delta c-5 \delta f)}\left(( a - b ) t \left(a\left(-9+\delta c^{2}+8 \delta f-4 \delta c \delta f\right)+\right.\right. \\
\left.\left.\left.b\left(-9+\delta c^{2}+8 \delta f-4 \delta c \delta f\right)-2\left(3 \delta c+\delta c^{2}+4 \delta f-2 \delta c \delta f\right)\right)+(9+3 \delta c-10 \delta f) p_{B}\right)\right\}
\end{array}\right\}
\end{aligned}
$$

The function might be outside the range, $(a+b) / 4<z<(2+a+b) / 4$.

We derive the condition that the reaction function is indeed in the range, $(a+b) / 4<z<(2+a+b) / 4$.

We have already obtained the condition that $\mathrm{z}$ is between $(\mathrm{a}+\mathrm{b}) / 4<\mathrm{z}<(2+\mathrm{a}+\mathrm{b}) / 4$ as follows:

$p_{B}+\frac{(b-a) t(3(a+b)(1-\delta c)-2(3-\delta c))}{6}<p_{A}<p_{B}+\frac{(b-a) t(3(a+b)(1-\delta c)+4 \delta c)}{6}$

If the following outcomes are positive, the reaction function satisfies the above inequalities: 


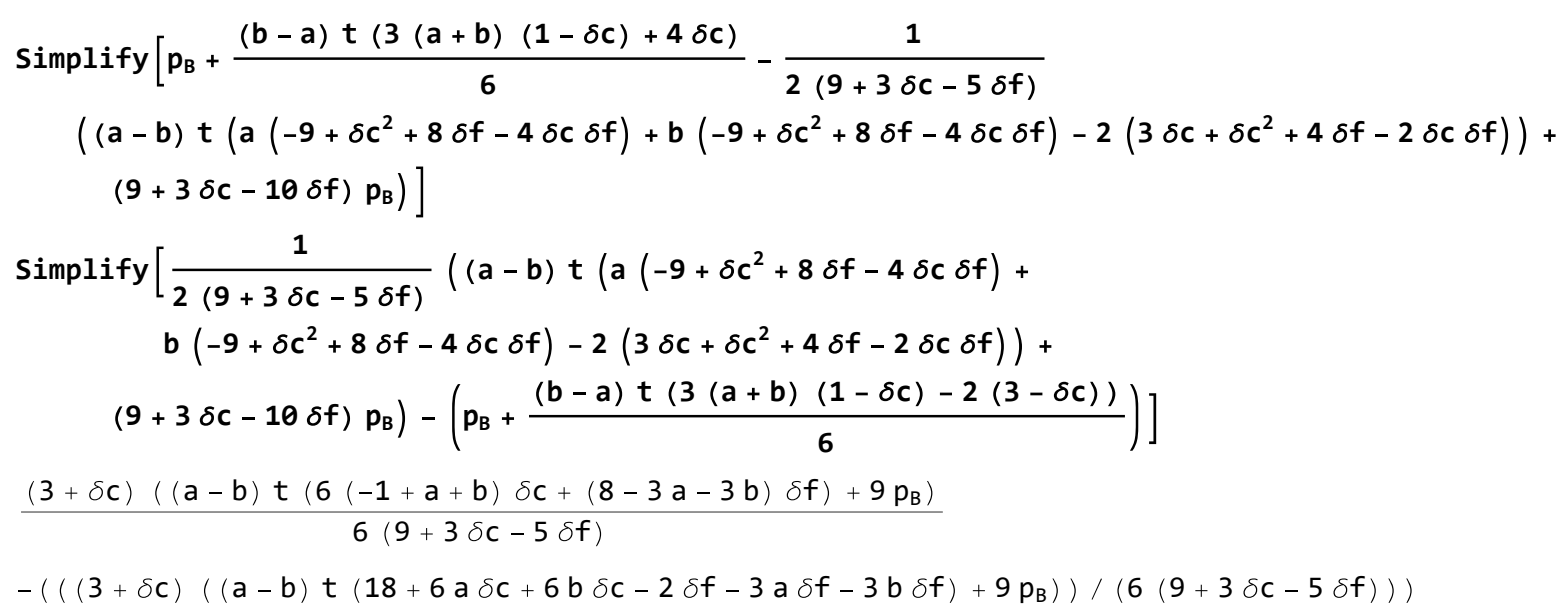

We derive the threshold values of $p_{A}$ such that each of the outcomes equals zero:

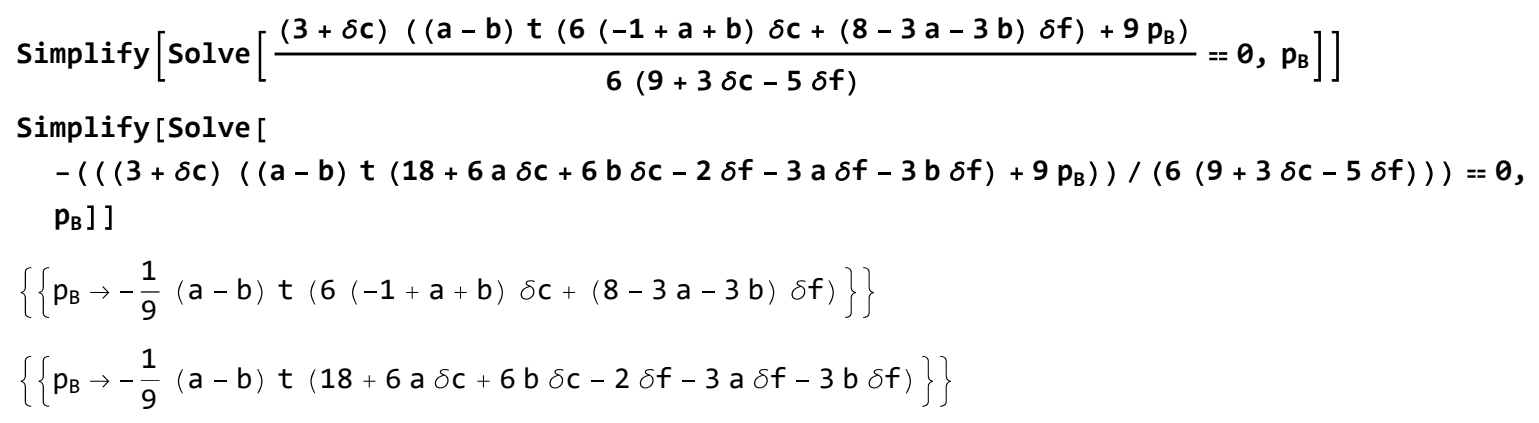

Therefore, if $p_{B}$ satisfies the following inequalities, the reaction function of Firm $\mathrm{B}$ is in the range, $(\mathrm{a}+\mathrm{b}) / 4<\mathrm{z}<(2+\mathrm{a}+\mathrm{b}) / 4$.

$\frac{(b-a) t(6(-1+a+b) \delta c+(8-3 a-3 b) \delta f)}{9}<p_{B}<\frac{(b-a) t(3(a+b)(2 \delta c-\delta f)+2(9-\delta f))}{9}$

Similarly, using the above outcomes, we derive the first-order derivative of Firm B's profit with respect to $p_{B}$

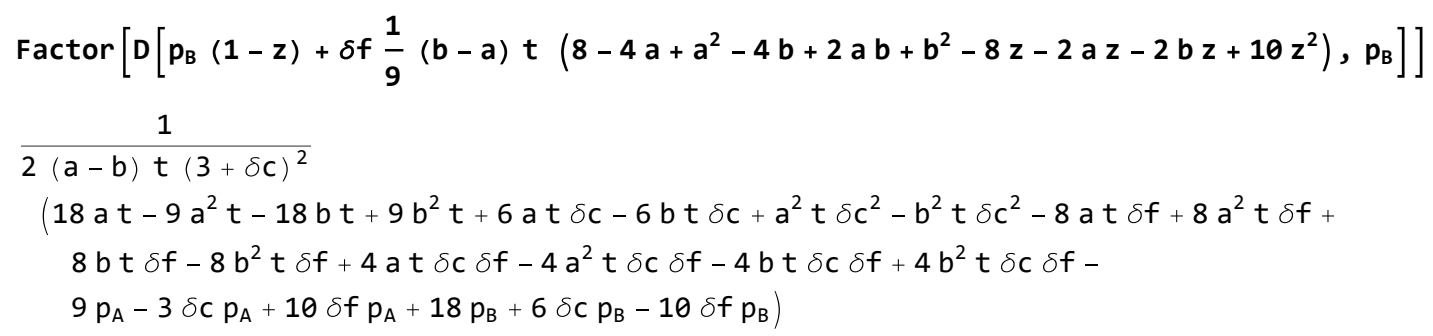

Using the derivative, we obtain the reaction function of Firm $\mathrm{B}$ in the range, $(\mathrm{a}+\mathrm{b}) / 4<\mathrm{z}<(2+\mathrm{a}+\mathrm{b}) / 4$.

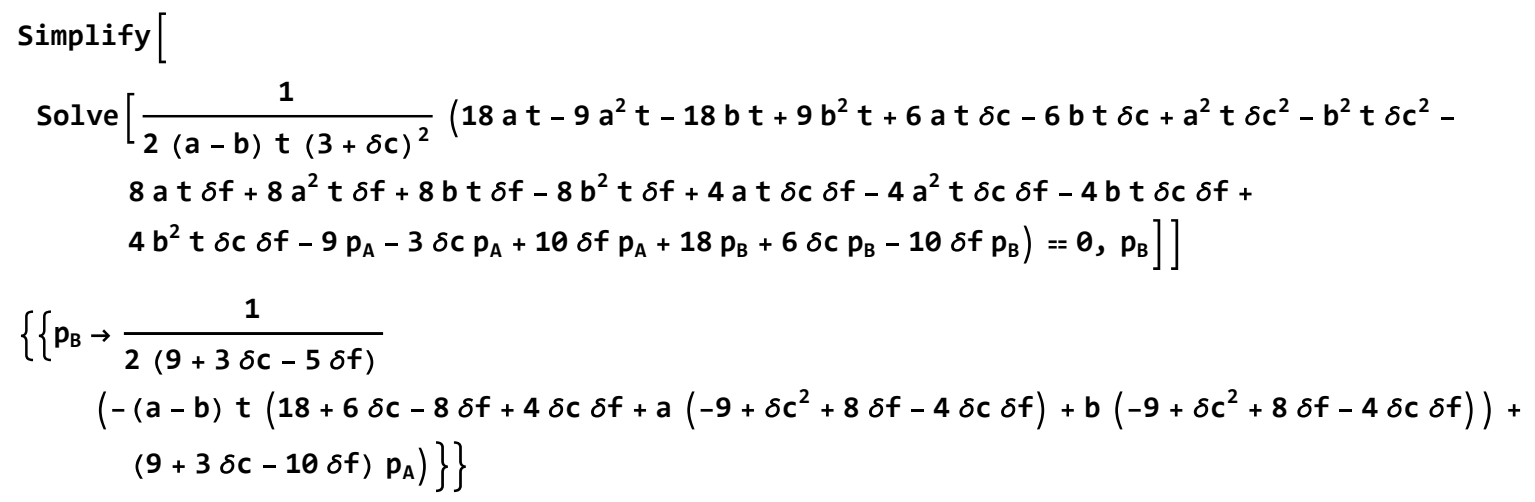


We have already obtained the condition that $z$ is between $(a+b) / 4$ and $(2+a+b) / 4$ as follows: $\mathrm{p}_{\mathrm{B}}+\frac{(\mathrm{b}-\mathrm{a}) \mathrm{t}(3(\mathrm{a}+\mathrm{b})(1-\delta \mathrm{c})-2(3-\delta c))}{6}<\mathrm{p}_{\mathrm{A}}<\mathrm{p}_{\mathrm{B}}+\frac{(\mathrm{b}-\mathrm{a}) \mathrm{t}(3(\mathrm{a}+\mathrm{b})(1-\delta \mathrm{c})+4 \delta \mathrm{c})}{6}$

which can be rewritten as $p_{A}-\frac{(b-a) t(3(a+b)(1-\delta c)+4 \delta c)}{6}<p_{B}<p_{A}-\frac{(b-a) t(3(a+b)(1-\delta c)-2(3-\delta c))}{6}$

If the following outcomes are positive, the reaction function satisfies the above inequalities:

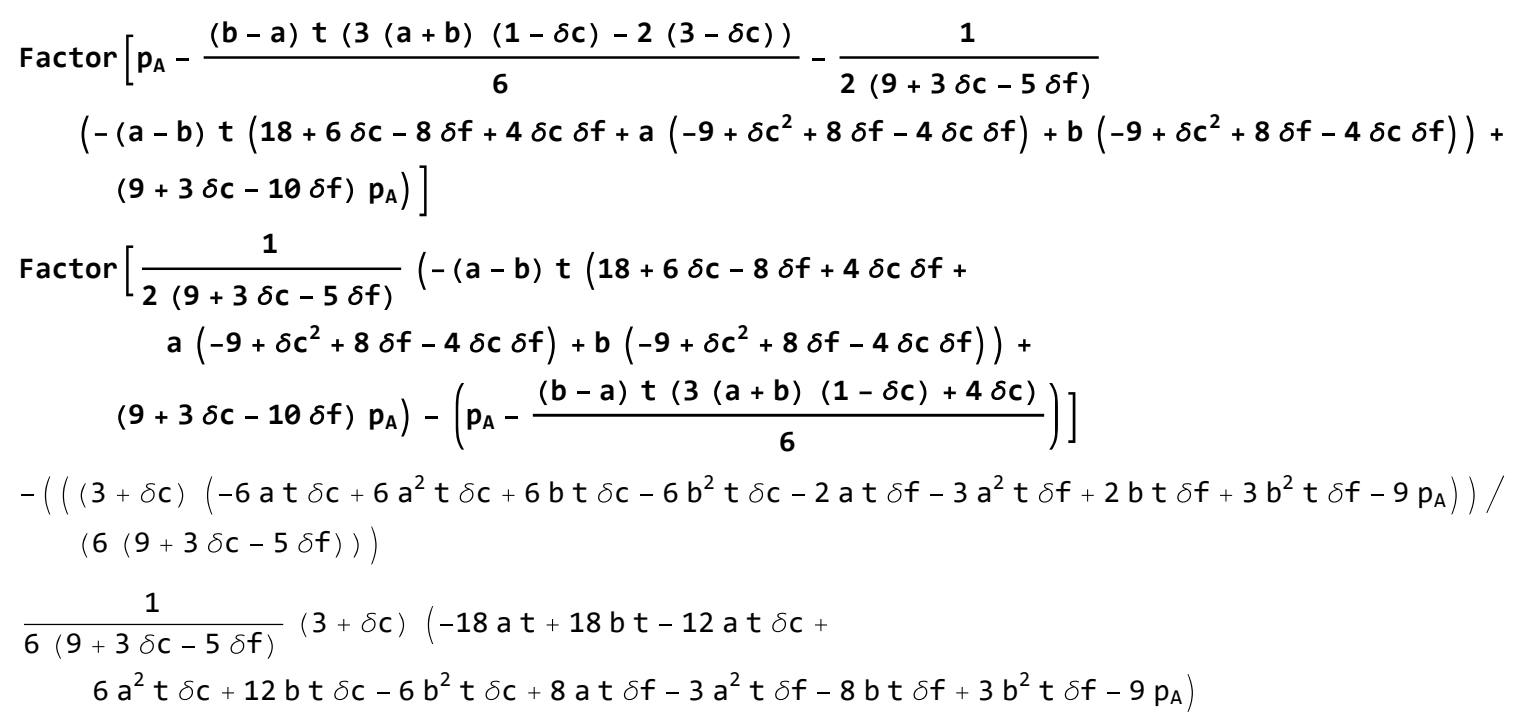

We derive the threshold values of $p_{A}$ such that each of the outcomes equals zero:

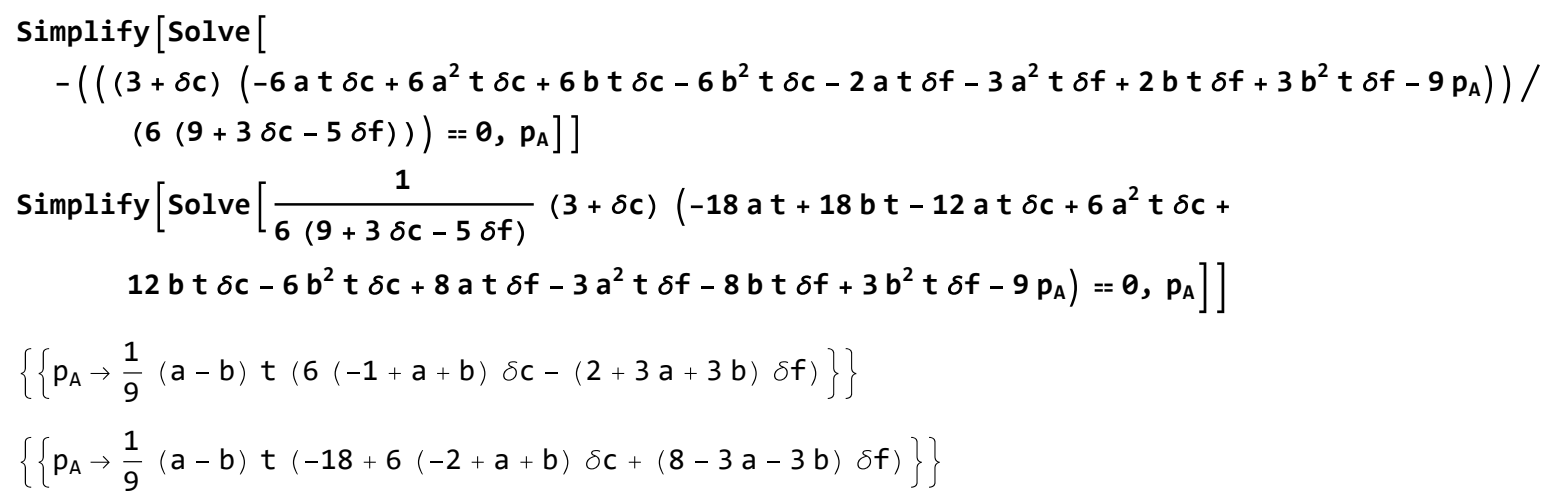

Therefore, if $p_{A}$ satisfies the following inequalities, the reaction function of Firm $\mathrm{B}$ is in the range, $(\mathrm{a}+\mathrm{b}) / 4<\mathrm{z}<(2+\mathrm{a}+\mathrm{b}) / 4$

$\frac{(b-a) t(6(1-a-b) \delta c+(2+3 a+3 b) \delta f)}{9}<p_{A}<\frac{(b-a) t(18+6(2-a-b) \delta c-(8-3 a-3 b) \delta f)}{9}$

(Case iii) $(2+\mathrm{a}+\mathrm{b}) / 4 \leq \mathrm{z} \leq 1$

The following prices are the second period prices.

$\left\{p_{A 0} \rightarrow-\frac{1}{3}(a-b) t(a+b+2 z), p_{A n} \rightarrow \theta, p_{B O} \rightarrow(a-b) t(a+b-2 z), p_{B n} \rightarrow \frac{1}{3}(a-b) t(a+b-4 z)\right\}$

Anticipating the second period prices, consumers choose one of the first-period prices $p_{A}$ or $p_{B}$ ( $p_{A}$ is the first-period price of firm $\mathrm{A}$ and $p_{B}$ is the first-period price of firm $\mathrm{B}$ )

The location of the indifferent consumer, $z$, is derived by the following equation: 


$$
\begin{aligned}
& \text { Solve }\left[-p_{A}-t(z-a)^{2}-\delta c\left(\frac{1}{3}(a-b) t(a+b-4 z)+t(z-b)^{2}\right)=\right. \\
& \left.-p_{B}-t(z-b)^{2}-\delta c\left(((a-b) t(a+b-2 z))+t(z-b)^{2}\right), z\right] \\
& \left\{\left\{z \rightarrow \frac{-3 a^{2} t+3 b^{2} t+2 a^{2} t \delta c-2 b^{2} t \delta c-3 p_{A}+3 p_{B}}{2(a-b) t(-3+\delta c)}\right\}\right\}
\end{aligned}
$$

We set the location of the indifferent consumer $z$ :

$$
\begin{aligned}
& z=\frac{-3 a^{2} t+3 b^{2} t+2 a^{2} t \delta c-2 b^{2} t \delta c-3 p_{A}+3 p_{B}}{2(a-b) t(-3+\delta c)} \\
& \frac{-3 a^{2} t+3 b^{2} t+2 a^{2} t \delta c-2 b^{2} t \delta c-3 p_{A}+3 p_{B}}{2(a-b) t(-3+\delta c)}
\end{aligned}
$$

We derive the condition that the location of the indifferent consumer, $z$, is between $(2+a+b) / 4$ and 1 , by solving the following equations

$$
\begin{aligned}
& \text { Factor [Solve } \left.\left[z-(2+a+b) / 4=0, p_{A}\right]\right] \\
& \text { Factor [Solve } \left.\left[1-z=0, p_{A}\right]\right] \\
& \left\{\left\{p_{A} \rightarrow \frac{1}{6}\left(6 a t-3 a^{2} t-6 b t+3 b^{2} t-2 a t \delta c+3 a^{2} t \delta c+2 b t \delta c-3 b^{2} t \delta c+6 p_{B}\right)\right\}\right\} \\
& \left\{\left\{p_{A} \rightarrow \frac{1}{3}\left(6 a t-3 a^{2} t-6 b t+3 b^{2} t-2 a t \delta c+2 a^{2} t \delta c+2 b t \delta c-2 b^{2} t \delta c+3 p_{B}\right)\right\}\right\}
\end{aligned}
$$

By simplifying the above values of $p_{A}$, we have the condition that $\mathrm{z}$ is between $(2+\mathrm{a}+\mathrm{b}) / 4$ and 1 as follows:

$\mathrm{p}_{\mathrm{B}}+\frac{(\mathrm{b}-\mathrm{a}) \mathrm{t}((\mathrm{a}+\mathrm{b})(3-2 \delta \mathrm{c})-2(3-\delta \mathrm{c}))}{3} \leq \mathrm{p}_{\mathrm{A}} \leq \mathrm{p}_{\mathrm{B}}+\frac{(\mathrm{b}-\mathrm{a}) \mathrm{t}(3(\mathrm{a}+\mathrm{b})(1-\delta \mathrm{c})-2(3-\delta \mathrm{c}))}{6}$

We have already derived the 2nd period profits of Firms A and B as follows:

$\pi_{A 2}:-\frac{1}{18}(a-b) t(a+b+2 z)^{2}$

$\pi_{B 2}:-\frac{1}{18}(a-b) t\left(-18 a+a^{2}-18 b+2 a b+b^{2}+36 z+10 a z+10 b z-20 z^{2}\right)$

The first-order condition of Firm A with respect to $p_{A}$ is

$$
\begin{aligned}
& \text { Factor }\left[D\left[p_{A} z+\delta f\left(\frac{1}{18}(b-a) t(a+b+2 z)^{2}\right), p_{A}\right]\right] \\
& \frac{1}{2(a-b) t(-3+\delta c)^{2}}\left(9 a^{2} t-9 b^{2} t-9 a^{2} t \delta c+9 b^{2} t \delta c+2 a^{2} t \delta c^{2}-2 b^{2} t \delta c^{2}-4 a^{2} t \delta f+\right. \\
& \left.4 b^{2} t \delta f+2 a^{2} t \delta c \delta f-2 b^{2} t \delta c \delta f+18 p_{A}-6 \delta c p_{A}-2 \delta f p_{A}-9 p_{B}+3 \delta c p_{B}+2 \delta f p_{B}\right)
\end{aligned}
$$

The reaction function of Firm A within the range in which $(2+a+b) / 4 \leq \mathrm{z} \leq 1$ is

$$
\begin{aligned}
& \text { Simplify }[ \\
& \text { Solve }\left[\frac { 1 } { 2 ( a - b ) t ( - 3 + \delta c ) ^ { 2 } } \left(9 a^{2} t-9 b^{2} t-9 a^{2} t \delta c+9 b^{2} t \delta c+2 a^{2} t \delta c^{2}-2 b^{2} t \delta c^{2}-4 a^{2} t \delta f+\right.\right. \\
& \left.\left.\left.\quad 4 b^{2} t \delta f+2 a^{2} t \delta c \delta f-2 b^{2} t \delta c \delta f+18 p_{A}-6 \delta c p_{A}-2 \delta f p_{A}-9 p_{B}+3 \delta c p_{B}+2 \delta f p_{B}\right)=0, p_{A}\right]\right] \\
& \left\{\left\{p_{A} \rightarrow\left(\left(a^{2}-b^{2}\right) t\left(9+2 \delta c^{2}-4 \delta f+\delta c(-9+2 \delta f)\right)+(-9+3 \delta c+2 \delta f) p_{B}\right) /(2(-9+3 \delta c+\delta f))\right\}\right\} \\
& p_{A} \rightarrow \frac{(9-3 \delta c-2 \delta f) p_{B}}{2(9-3 \delta c-\delta f)}+\frac{(b-a)(a+b) t((3-\delta c)(3-2 \delta c)-2(2-\delta c) \delta f)}{2(9-3 \delta c-\delta f)}
\end{aligned}
$$

The function might be outside the range, $(2+\mathrm{a}+\mathrm{b}) / 4 \leq \mathrm{z} \leq 1$.

We derive the condition that the reaction function is indeed in the range, $(2+a+b) / 4 \leq z \leq 1$.

We have already obtained the condition that $\mathrm{z}$ is between $(2+\mathrm{a}+\mathrm{b}) / 4$ and 1 as follows: 
$p_{B}+\frac{(b-a) t((a+b)(3-2 \delta c)-2(3-\delta c))}{3} \leq p_{A} \leq p_{B}+\frac{(b-a) t(3(a+b)(1-\delta c)-2(3-\delta c))}{6}$

If $p_{B}$ is larger than $\mathrm{p}_{\mathrm{A}}-\frac{(\mathrm{b}-\mathrm{a}) \mathrm{t}((\mathrm{a}+\mathrm{b})(3-2 \delta \mathrm{c})-2(3-\delta \mathrm{c}))}{3}, z$ becomes 1 .

In this case, Firm A chooses the following $p_{A}$ which just leads to $\mathrm{z}=1$.

$p_{A} \rightarrow p_{B}+\frac{(b-a) t((a+b)(3-2 \delta c)-2(3-\delta c))}{3}$.

The reaction function is within the range $(2+a+b) / 4 \leq \mathrm{Z} \leq 1$ if the following are positive:

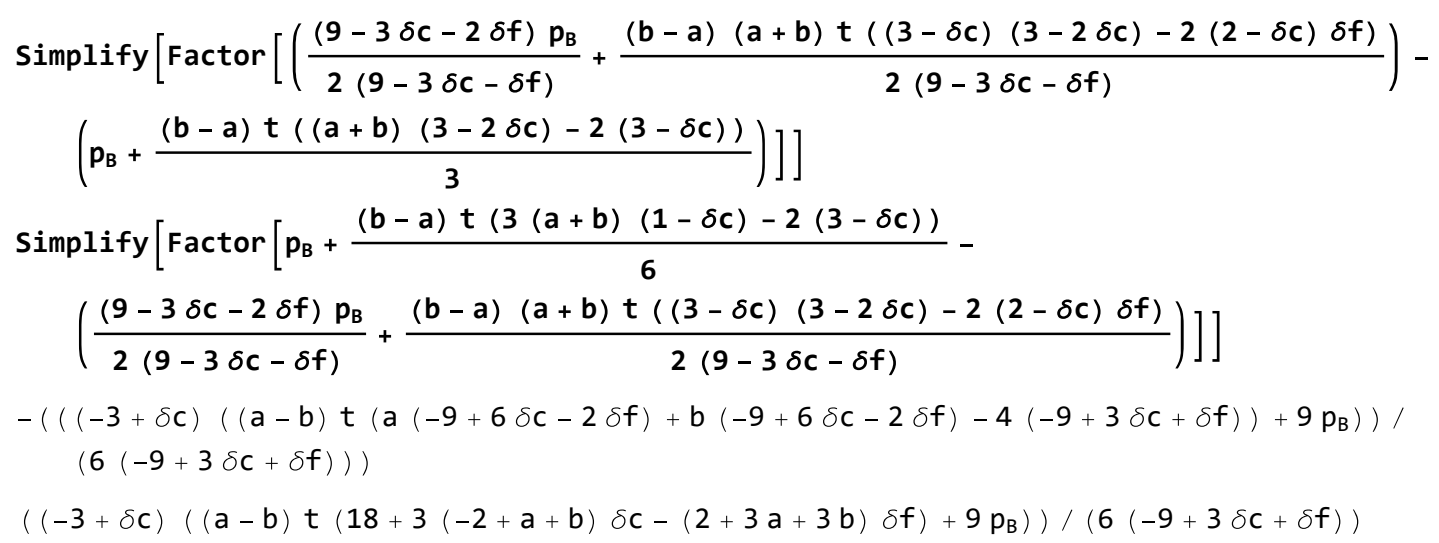

Therefore, the reaction function of Firm $A$ is within the range $(2+a+b) / 4 \leq z \leq 1$ if $\frac{(b-a) t(4(9-3 \delta c-\delta f)-(a+b)(9-6 \delta c+2 \delta f))}{9} \geq$ $\mathrm{p}_{B} \geq \frac{(b-a) t(2(9-3 \delta c-\delta f)+3(a+b)(\delta c-\delta f))}{9}$

Note that if $p_{B}$ is larger than the left-hand side value of the inequality, Firm A chooses the following $p_{A}$, which leads to $\mathrm{z}=1$.

$p_{A} \rightarrow p_{B}+\frac{(b-a) t((a+b)(3-2 \delta c)-2(3-\delta c))}{3}$.

Similarly, using the above outcomes, we derive the first-order condition of Firm B with respect to $p_{B}$

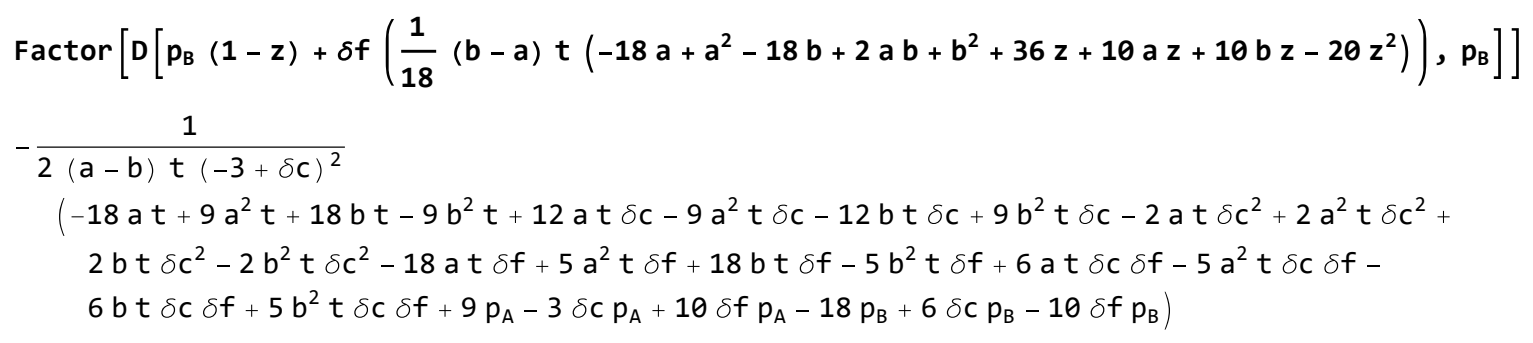

The reaction function of Firm $B$ is 


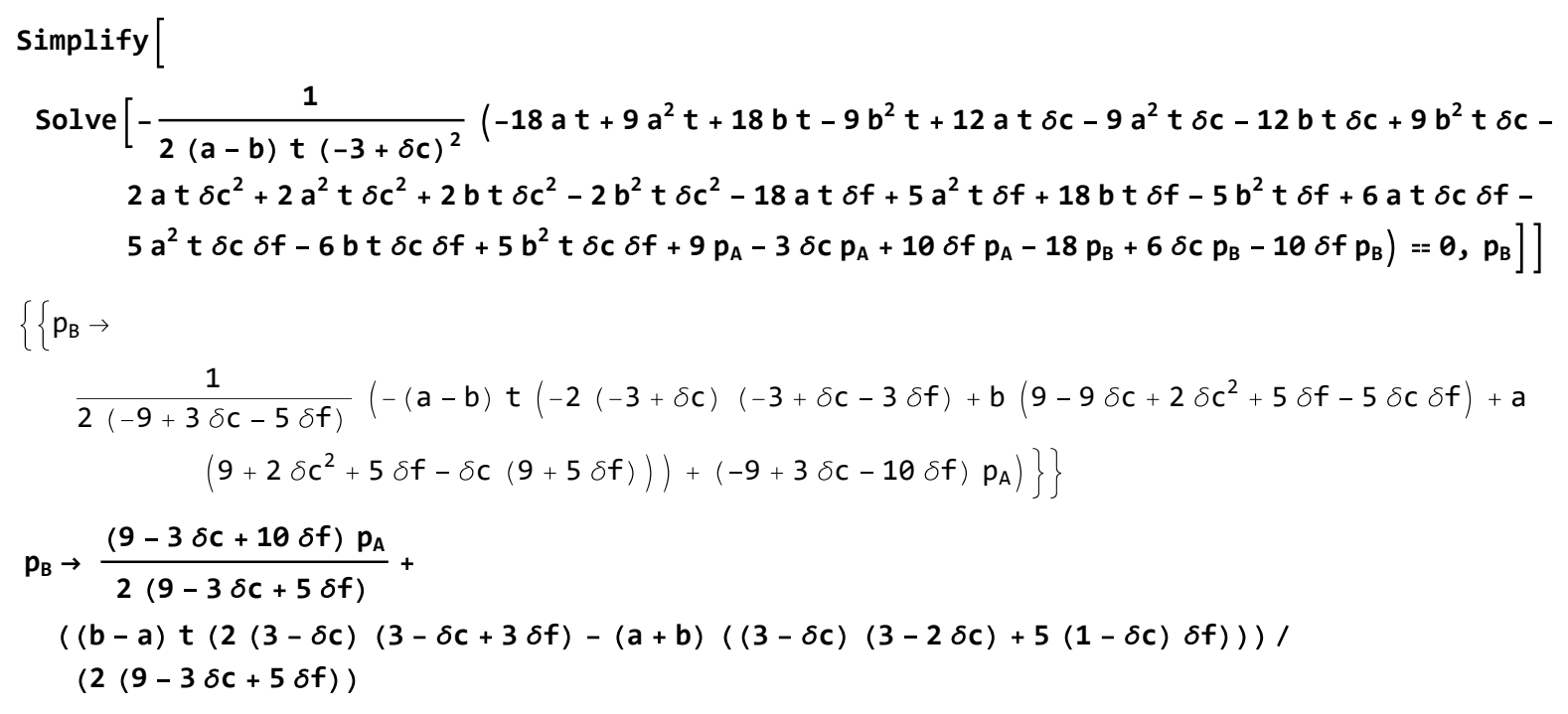

We check the condition that the reaction function of Firm B is within the range $(2+a+b) / 4 \leq Z \leq$ 1 .

$p_{B}+\frac{(b-a) t((a+b)(3-2 \delta c)-2(3-\delta c))}{3}<p_{A}<p_{B}+\frac{(b-a) t(3(a+b)(1-\delta c)-2(3-\delta c))}{6}$

Note that if $p_{B}$ is larger than $\mathrm{p}_{\mathrm{A}}-\frac{(\mathrm{b}-\mathrm{a}) \mathrm{t}((\mathrm{a}+\mathrm{b})(3-2 \delta \mathrm{c})-2(3-\delta \mathrm{c}))}{3}, z$ becomes 1 .

The reaction function of Firm $B$ is within the range in which $(2+a+b) / 4 \leq \mathrm{z} \leq 1$ if the following are positive:

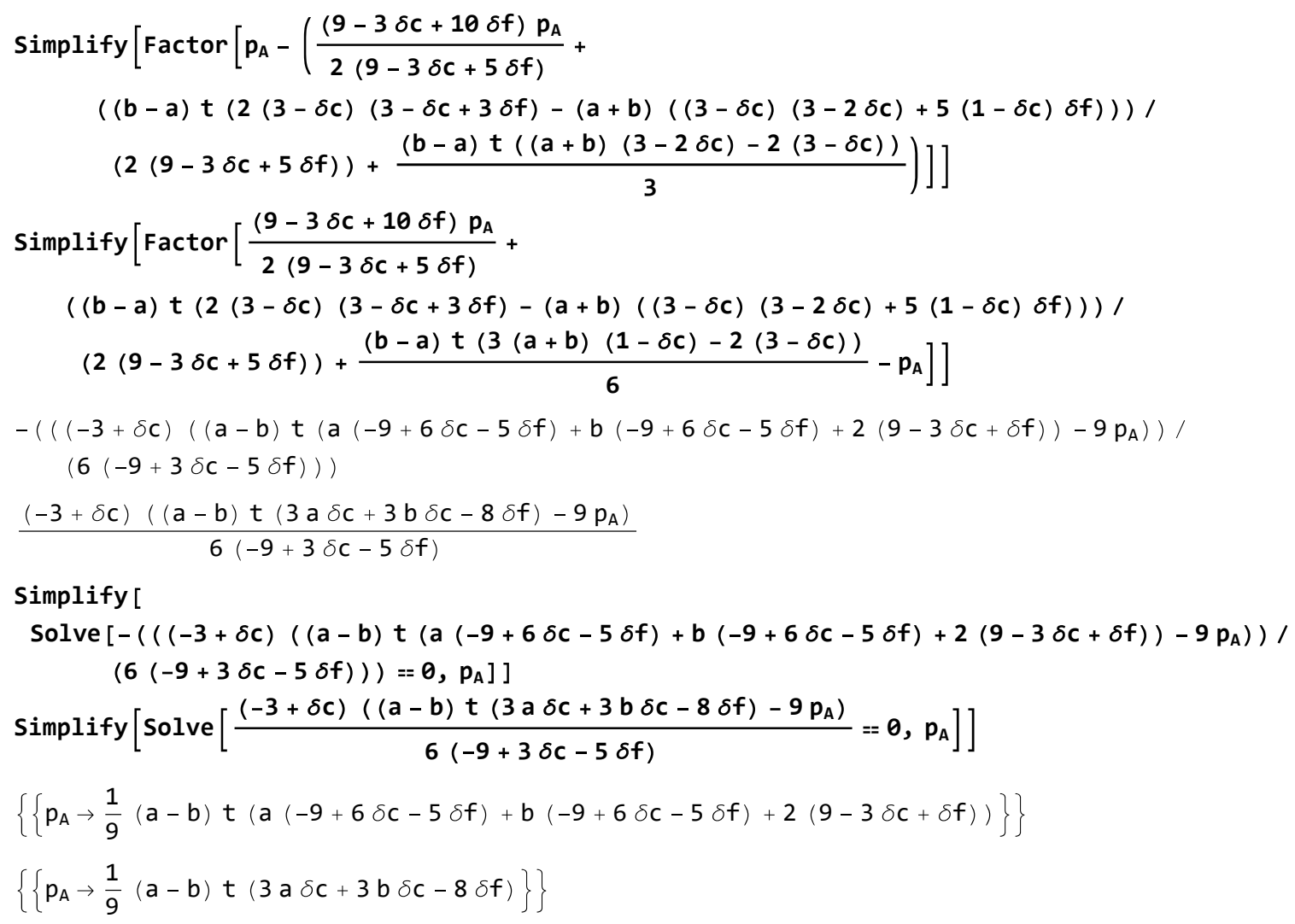

Thus, the reaction function of Firm $B$ is within the range $(2+a+b) / 4 \leq z \leq 1$ if

$\frac{(b-a) t((a+b)(9-6 \delta c+5 \delta f)-2(9-3 \delta c+\delta f))}{9} \leq p_{A} \leq \frac{(b-a) t(8 \delta f-3(a+b) \delta c)}{9}$ 
We summarize below the results from our analysis of the reaction functions in the three cases.

(Case i) $0 \leq \mathrm{z} \leq(\mathrm{a}+\mathrm{b}) / 4$.

Firm A's reaction function in this range is

$p_{A} \rightarrow\left((b-a) t\left(6 \delta c-2 \delta c^{2}+8 \delta f+4 \delta c \delta f+(a+b)\left(9-9 \delta c+2 \delta c^{2}+5 \delta f-5 \delta c \delta f\right)\right)+\right.$

$\left.(9-3 \delta c+10 \delta f) p_{B}\right) /(2(9-3 \delta c+5 \delta f))$

This is applicable for the following range of $p_{B}$

$-\frac{1}{9}(b-a) t((a+b)(9-6 \delta c+5 \delta f)+6 \delta c-8 \delta f)<p_{B}<\frac{(b-a) t(3(-2+a+b) \delta c+8 \delta f)}{9}$

We check the endpoints of Firm A's reaction function in the range of $p_{B}$ (see the inequalities above). Substituting the minimum and maximum values of $p_{B}$ into Firm A's reaction function, we obtain the endpoints (vectors) of Firm A's reaction function in the $\left(p_{A}, p_{B}\right)$ coordinate system:

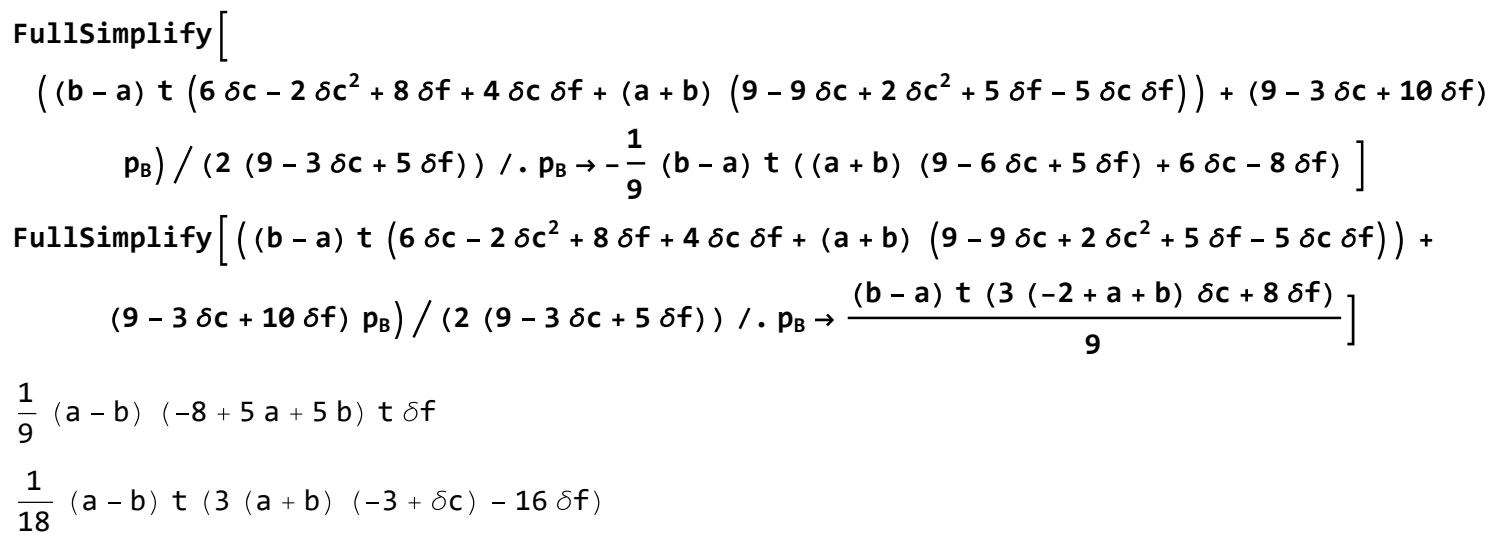

Firm A's reaction function in this range consists of the line segment between the following two points:

$$
\begin{aligned}
& \left(\frac{1}{9}(a-b)(-8+5 a+5 b) t \delta f,-\frac{1}{9}(b-a) t((a+b)(9-6 \delta c+5 \delta f)+6 \delta c-8 \delta f)\right) \\
& \left(\frac{1}{18}(a-b) t(3(a+b)(-3+\delta c)-16 \delta f), \frac{(b-a) t(3(-2+a+b) \delta c+8 \delta f)}{9}\right)
\end{aligned}
$$

Firm B's reaction function in this range is

$p_{B} \rightarrow \frac{1}{2(9-3 \delta c-\delta f)}\left(((b-a)(2-a-b)) t\left(9+2 \delta c^{2}-4 \delta f+\delta c(-9+2 \delta f)\right)+(9-3 \delta c-2 \delta f) p_{A}\right)$

This is applicable for the following range of $p_{A}$

$\frac{(b-a) t(18+(a+b)(9-6 \delta c+2 \delta f)-8 \delta f)}{9} \geq p_{A} \geq \frac{(b-a) t(18-3(a+b)(\delta c-\delta f)-8 \delta f)}{9}$

Note that if $p_{A}$ is larger than the left-hand side value of the inequality, Firm B chooses the following $p_{B}$, which just leads to $\mathrm{z}=0$.

$p_{B} \rightarrow p_{A}-\frac{(b-a) t((a+b)(3-2 \delta c)+2 \delta c)}{3}$

We check the endpoints of Firm B's reaction function in the range of $p_{A}$, based on the above inequalities. Substituting the minimum and maximum values of $p_{A}$ into Firm B's reaction 
function, we obtain the endpoints (vectors) of Firm B's reaction function in the $\left(p_{A}, p_{B}\right)$ coordinate system:

$$
\begin{aligned}
& \text { Fullsimplify }[ \\
& \frac{1}{2(9-3 \delta c-\delta f)}\left(((b-a)(2-a-b)) t\left(9+2 \delta c^{2}-4 \delta f+\delta c(-9+2 \delta f)\right)+(9-3 \delta c-2 \delta f) p_{A}\right) / \\
& \left.p_{A} \rightarrow \frac{(b-a) t(18-3(a+b)(\delta c-\delta f)-8 \delta f)}{9}\right] \\
& \text { Fullsimplify }\left[\frac { 1 } { 2 ( 9 - 3 \delta c - \delta f ) } \left(((b-a)(2-a-b)) t\left(9+2 \delta c^{2}-4 \delta f+\delta c(-9+2 \delta f)\right)+\right.\right. \\
& \left.\left.\quad(9-3 \delta c-2 \delta f) p_{A}\right) / \cdot p_{A} \rightarrow \frac{(b-a) t(18+(a+b)(9-6 \delta c+2 \delta f)-8 \delta f)}{9}\right] \\
& -\frac{1}{18}(a-b) t(3(-4+a+b)(-3+\delta c)+2(-8+3 a+3 b) \delta f) \\
& -\frac{2}{9}(a-b) t(9-3 \delta c+(-4+a+b) \delta f)
\end{aligned}
$$

Firm B's reaction function in this range consists of the line segment between the following two points:

$$
\begin{aligned}
& \left(\frac{(b-a) t(18-3(a+b)(\delta c-\delta f)-8 \delta f)}{9},\right. \\
& \left.-\frac{1}{18}(a-b) t(3(-4+a+b)(-3+\delta c)+2(-8+3 a+3 b) \delta f)\right), \\
& \left(\frac{(b-a) t(18+(a+b)(9-6 \delta c+2 \delta f)-8 \delta f)}{9},-\frac{2}{9}(a-b) t(9-3 \delta c+(-4+a+b) \delta f)\right)
\end{aligned}
$$

Note that if the following inequality,

$$
\frac{(b-a) t(18+(a+b)(9-6 \delta c+2 \delta f)-8 \delta f)}{9} \leq p_{A} \text {, }
$$

holds, then Firm $\mathrm{B}$ chooses the following $p_{B}$, which just leads to $\mathrm{z}=0$.

$\mathrm{p}_{B} \rightarrow \mathrm{p}_{\mathrm{A}}-\frac{(\mathrm{b}-\mathrm{a}) \mathrm{t}((\mathrm{a}+\mathrm{b})(3-2 \delta \mathrm{c})+2 \delta \mathrm{c})}{3}$.

The reaction function of firm $\mathrm{B}$ leading to $\mathrm{z}=0$ consists of the line segment connecting the following two points.

$$
\begin{aligned}
& \left(\frac{(b-a) t(18+(a+b)(9-6 \delta c+2 \delta f)-8 \delta f)}{9},-\frac{2}{9}(a-b) t(9-3 \delta c+(-4+a+b) \delta f)\right), \\
& \left(\frac{(b-a) t(18+(a+b)(9-6 \delta c+2 \delta f)-8 \delta f)}{9}+k,-\frac{2}{9}(a-b) t(9-3 \delta c+(-4+a+b) \delta f)+k\right),
\end{aligned}
$$

where $k$ is a (sufficiently large) positive constant.

(Case ii) $(\mathrm{a}+\mathrm{b}) / 4<\mathrm{z}<(2+\mathrm{a}+\mathrm{b}) / 4$

Firm A's reaction function in this range is

$p_{A} \rightarrow\left((9+3 \delta c-10 \delta f) p_{B}+(b-a) t\left((a+b)\left(9-\delta c^{2}-8 \delta f+4 \delta c \delta f\right)+2\left(3 \delta c+\delta c^{2}+4 \delta f-2 \delta c \delta f\right)\right)\right) /$ $(2(9+3 \delta c-5 \delta f))$

This is applicable for the following range of $p_{B}$ $\frac{(b-a) t(3(a+b)(2 \delta c-\delta f)-2(3 \delta c-4 \delta f))}{9}<p_{B}<\frac{(b-a) t(3(a+b)(2 \delta c-\delta f)+2(9-\delta f))}{9}$

We check the endpoints of Firm A's reaction function in the range of $p_{B}$ (see the inequalities above). Substituting the minimum and maximum values of $p_{B}$ into Firm A's reaction function, 
we obtain the endpoints (vectors) of Firm A's reaction function in the $\left(p_{A}, p_{B}\right)$ coordinate system:

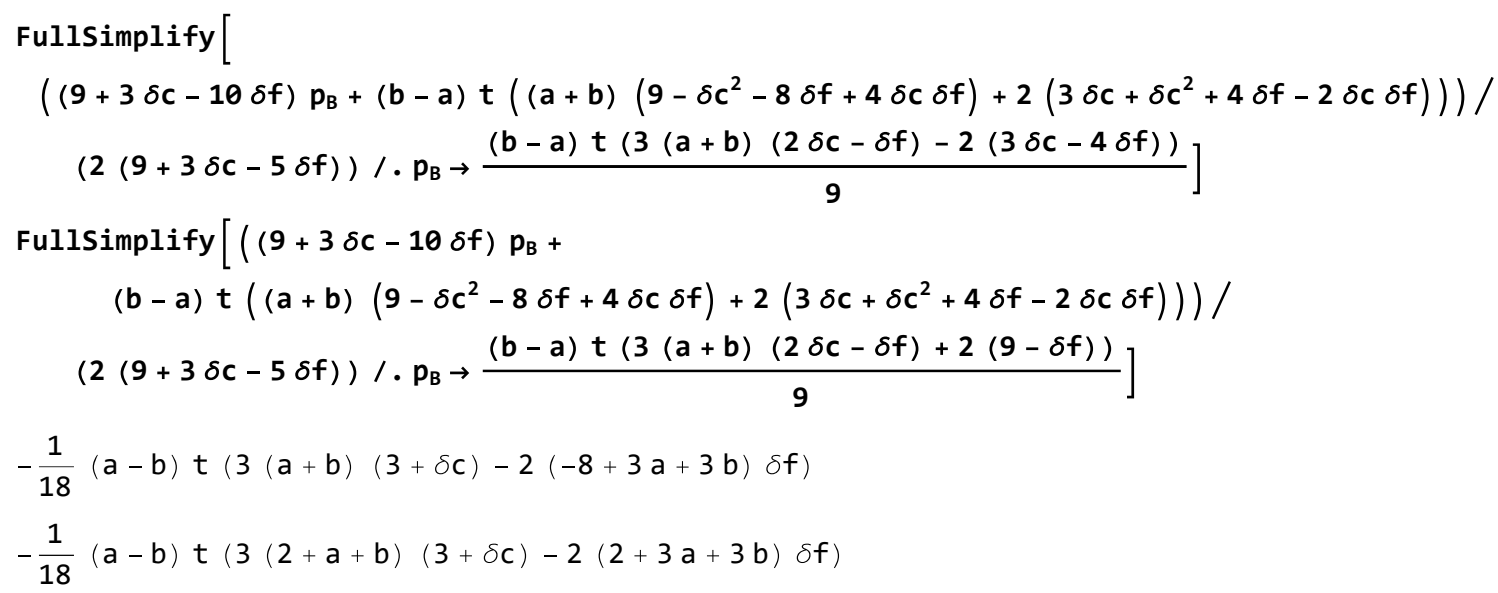

Firm A's reaction function in this range consists of the line segment between the following two points:

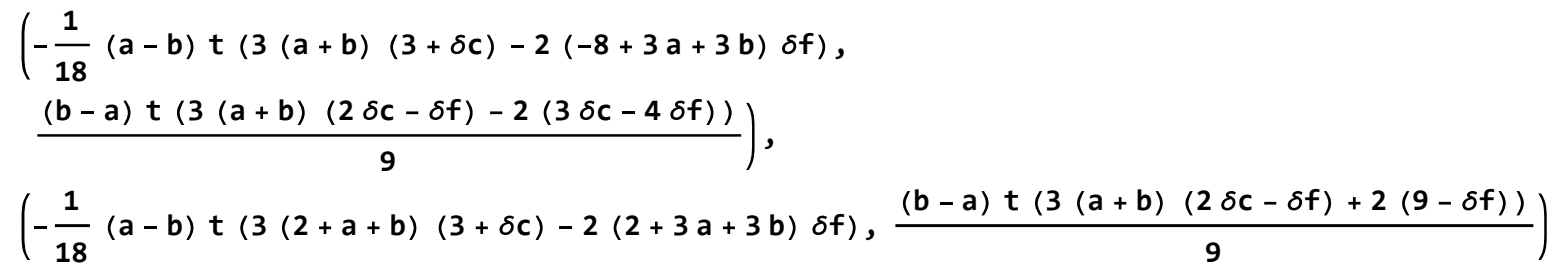

Firm B's reaction function in this range is $\mathrm{p}_{\mathrm{B}} \rightarrow\left((\mathrm{b}-\mathrm{a}) \mathrm{t}\left(2(9+3 \delta \mathrm{c}-4 \delta f+2 \delta \mathrm{c} \delta \mathrm{f})+(\mathrm{a}+\mathrm{b})\left(-9+\delta \mathrm{c}^{2}+8 \delta f-4 \delta c \delta f\right)\right)+(9+3 \delta c-10 \delta f) \mathrm{p}_{\mathrm{A}}\right) /$ $(2(9+3 \delta c-5 \delta f))$

This is applicable for the following range of $p_{A}$

$\frac{(b-a) t(6(1-a-b) \delta c+(2+3 a+3 b) \delta f)}{9}<p_{A}<\frac{(b-a) t(18+6(2-a-b) \delta c-(8-3 a-3 b) \delta f)}{9}$

We check the endpoints of Firm B's reaction function in the range of $p_{A}$ (see the inequalities above). Substituting the minimum and maximum values of $p_{A}$ into Firm B's reaction function, we obtain the endpoints (vectors) of Firm B's reaction function in the $\left(p_{A}, p_{B}\right)$ coordinate system:

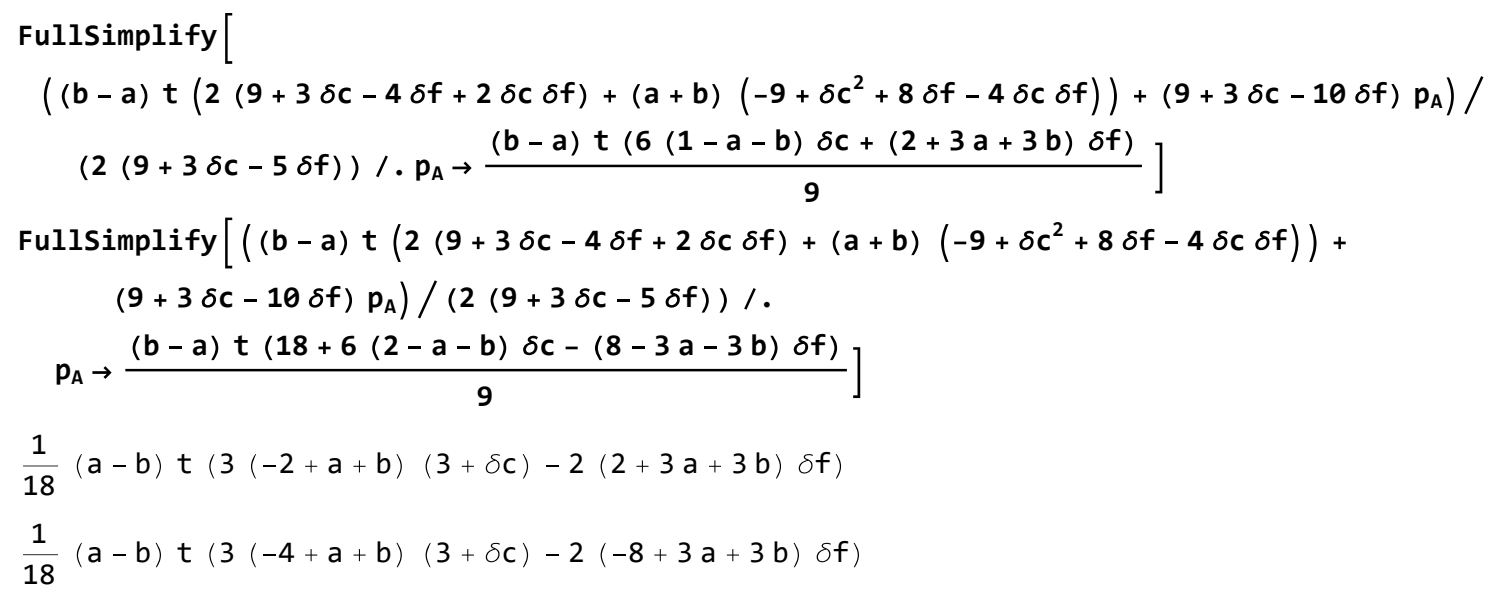

Firm B's reaction function in this range consists of the line segment between the following two points: 


$$
\begin{aligned}
& \left(\frac{(b-a) t(6(1-a-b) \delta c+(2+3 a+3 b) \delta f)}{9},\right. \\
& \left.\frac{1}{18}(a-b) t(3(-2+a+b)(3+\delta c)-2(2+3 a+3 b) \delta f)\right), \\
& \left(\frac{(b-a) t(18+6(2-a-b) \delta c-(8-3 a-3 b) \delta f)}{9},\right. \\
& \left.\frac{1}{18}(a-b) t(3(-4+a+b)(3+\delta c)-2(-8+3 a+3 b) \delta f)\right)
\end{aligned}
$$

(Case iii) $(2+\mathrm{a}+\mathrm{b}) / 4 \leq 1 \leq \mathrm{z}$

Firm A's reaction function in this range is

$p_{A} \rightarrow \frac{(9-3 \delta c-2 \delta f) p_{B}}{2(9-3 \delta c-\delta f)}+\frac{(b-a)(a+b) t((3-\delta c)(3-2 \delta c)-2(2-\delta c) \delta f)}{2(9-3 \delta c-\delta f)}$

This is applicable for the following range of $p_{B}$

$$
\begin{gathered}
\frac{(b-a) t(4(9-3 \delta c-\delta f)-(a+b)(9-6 \delta c+2 \delta f))}{9} \geq \\
p_{B} \geq \frac{(b-a) t(2(9-3 \delta c-\delta f)+3(a+b)(\delta c-\delta f))}{9}
\end{gathered}
$$

Note that if $p_{B}$ is larger than the left-hand side value of the inequality, Firm A chooses the following $p_{A}$, which leads to $\mathrm{z}=1$.

$\mathrm{p}_{\mathrm{A}} \rightarrow \mathrm{p}_{\mathrm{B}}+\frac{(\mathrm{b}-\mathrm{a}) \mathrm{t}((\mathrm{a}+\mathrm{b})(3-2 \delta \mathrm{c})-2(3-\delta \mathrm{c}))}{3}$.

We check the endpoints of Firm A's reaction function in the range of $p_{B}$ (see the inequalities above). Substituting the minimum and maximum values of $p_{B}$ into Firm A's reaction function, we obtain the endpoints (vectors) of Firm A's reaction function in the $\left(p_{A}, p_{B}\right)$ coordinate system:

$$
\begin{aligned}
& \text { Fullsimplify }\left[\frac{(9-3 \delta c-2 \delta f) p_{B}}{2(9-3 \delta c-\delta f)}+\frac{(b-a)(a+b) t((3-\delta c)(3-2 \delta c)-2(2-\delta c) \delta f)}{2(9-3 \delta c-\delta f)} /\right. \\
& \left.p_{B} \rightarrow \frac{(b-a) t(2(9-3 \delta c-\delta f)+3(a+b)(\delta c-\delta f))}{9}\right] \\
& \text { Fullsimplify }\left[\frac{(9-3 \delta c-2 \delta f) p_{B}}{2(9-3 \delta c-\delta f)}+\frac{(b-a)(a+b) t((3-\delta c)(3-2 \delta c)-2(2-\delta c) \delta f)}{2(9-3 \delta c-\delta f)} /\right. \\
& \left.p_{B} \rightarrow \frac{(b-a) t(4(9-3 \delta c-\delta f)-(a+b)(9-6 \delta c+2 \delta f))}{9}\right] \\
& \frac{1}{18}(a-b) t(3(2+a+b)(-3+\delta c)+2(2+3 a+3 b) \delta f) \\
& \frac{2}{9}(a-b) t(-9+3 \delta c+(2+a+b) \delta f)
\end{aligned}
$$

Firm A's reaction function in this range consists of the line segment between the following two points:

$$
\begin{aligned}
& \left(\frac{1}{18}(a-b) t(3(2+a+b)(-3+\delta c)+2(2+3 a+3 b) \delta f)\right. \\
& \left.\frac{(b-a) t(2(9-3 \delta c-\delta f)+3(a+b)(\delta c-\delta f))}{9}\right) \\
& \left(\frac{2}{9}(a-b) t(-9+3 \delta c+(2+a+b) \delta f), \frac{(b-a) t(4(9-3 \delta c-\delta f)-(a+b)(9-6 \delta c+2 \delta f))}{9}\right)
\end{aligned}
$$

Note that if the following inequality, 
$\frac{(b-a) t(4(9-3 \delta c-\delta f)-(a+b)(9-6 \delta c+2 \delta f))}{9} \leq p_{B}$,

holds, then Firm A chooses the following $p_{A}$, which leads to $\mathrm{z}=1$.

$p_{A} \rightarrow p_{B}+\frac{(b-a) t((a+b)(3-2 \delta c)-2(3-\delta c))}{3}$.

The reaction function of firm A leading to $\mathrm{z}=1$ consists of the line segment connecting the following two points.

$$
\begin{aligned}
& \left(\frac{2}{9}(a-b) t(-9+3 \delta c+(2+a+b) \delta f), \frac{(b-a) t(4(9-3 \delta c-\delta f)-(a+b)(9-6 \delta c+2 \delta f))}{9}\right), \\
& \left(\frac{2}{9}(a-b) t(-9+3 \delta c+(2+a+b) \delta f)+k, \frac{(b-a) t(4(9-3 \delta c-\delta f)-(a+b)(9-6 \delta c+2 \delta f))}{9}+k\right),
\end{aligned}
$$

where $k$ is a (sufficiently large) positive constant.

Firm B's reaction function in this range is

$$
\begin{aligned}
p_{B} \rightarrow & \frac{(9-3 \delta c+10 \delta f) p_{A}}{2(9-3 \delta c+5 \delta f)}+ \\
& ((b-a) t(2(3-\delta c)(3-\delta c+3 \delta f)-(a+b)((3-\delta c)(3-2 \delta c)+5(1-\delta c) \delta f))) / \\
& (2(9-3 \delta c+5 \delta f))
\end{aligned}
$$

This is applicable for the following range of $p_{A}$

$$
\frac{(b-a) t((a+b)(9-6 \delta c+5 \delta f)-2(9-3 \delta c+\delta f))}{9}<p_{A}<\frac{(b-a) t(8 \delta f-3(a+b) \delta c)}{9}
$$

We check the endpoints of Firm B's reaction function in the range of $p_{A}$ (see the inequalities above). Substituting the minimum and maximum values of $p_{A}$ into Firm B's reaction function, we obtain the endpoints (vectors) of Firm B's reaction function in the $\left(p_{A}, p_{B}\right)$ coordinate system:

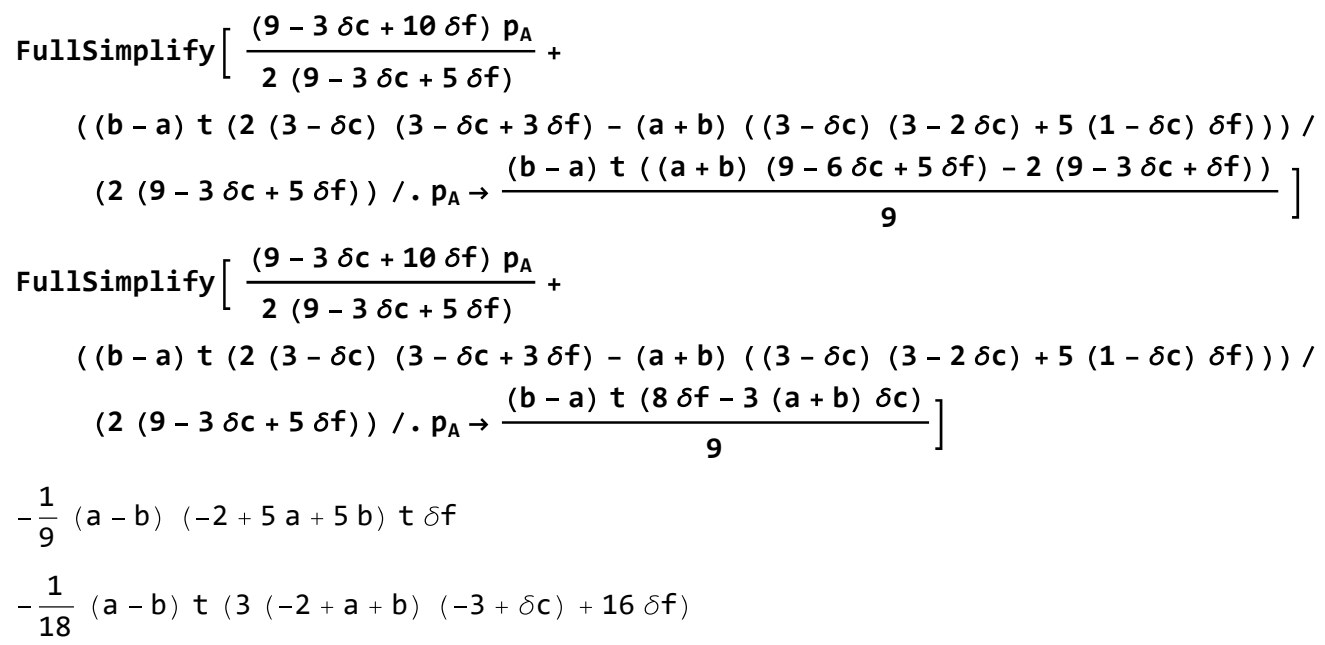

Firm B's reaction function in this range consists of the line segment between the following two points:

$$
\begin{aligned}
& \left(\frac{(b-a) t((a+b)(9-6 \delta c+5 \delta f)-2(9-3 \delta c+\delta f))}{9},-\frac{1}{9}(a-b)(-2+5 a+5 b) t \delta f\right), \\
& \left(\frac{(b-a) t(8 \delta f-3(a+b) \delta c)}{9},-\frac{1}{18}(a-b) t(3(-2+a+b)(-3+\delta c)+16 \delta f)\right)
\end{aligned}
$$


Because each firm's reaction function consists of three different pieces, we need to derive the 'true' reaction function by checking when the firm's profit obtains a global, rather than, local maximum.

From here on, we assume that the discount factors are common $\delta$. We set the following :

$\delta f=\delta$

$\delta$

$\delta \mathrm{c}=\delta$

$\delta$

From Firm A's reaction function derived above, we check the endpoints of each of the three line segments corresponding to the three cases: (i) $0 \leq \mathrm{z} \leq(\mathrm{a}+\mathrm{b}) / 4$, (ii) $(\mathrm{a}+\mathrm{b}) / 4<\mathrm{z}<(2+\mathrm{a}+\mathrm{b}) / 4$, (iii) $(2+a+b) / 4 \leq \mathrm{z} \leq 1$.

(Case i): The first endpoint below locates the left-hand side of Firm A's reaction function, and the second endpoint below locates the right-hand side of Firm A's reaction function in period 1.

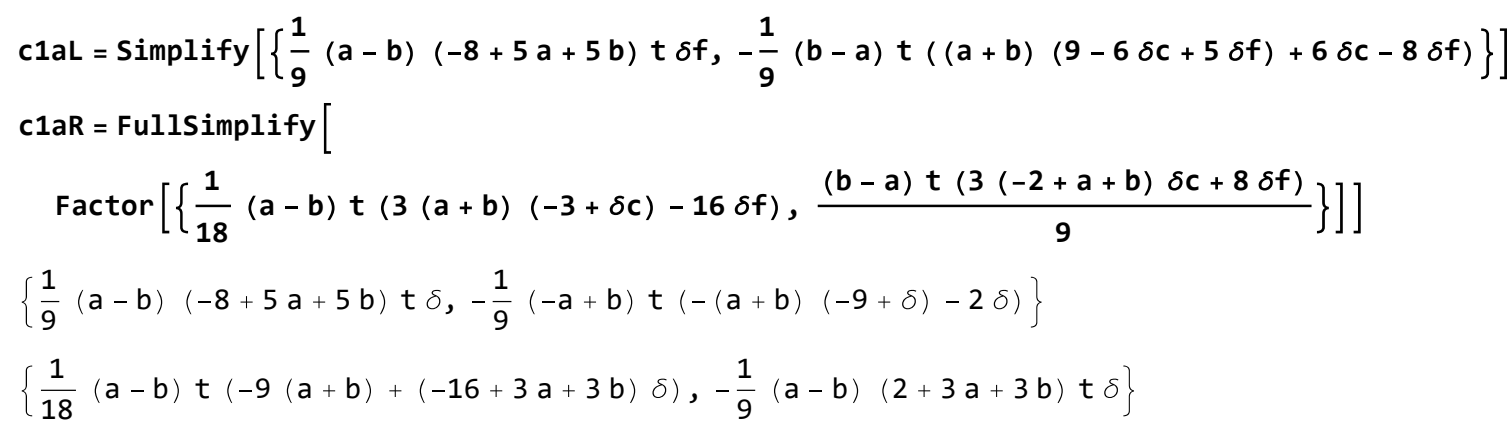

Case (ii): The first endpoint below locates the left-hand side of Firm A's reaction function, and the second endpoint below locates the right-hand side of Firm A's reaction function in period 1.

$$
\begin{aligned}
& \mathrm{c} 2 \mathrm{aL}=\text { FullSimplify }\left[\text { Factor } \left[\left\{-\frac{1}{18}(\mathrm{a}-\mathrm{b}) \mathrm{t}(3(\mathrm{a}+\mathrm{b})(3+\delta \mathrm{c})-2(-8+3 \mathrm{a}+3 \mathrm{~b}) \delta f),\right.\right.\right. \\
& \left.\left.\left.\frac{(b-a) t(3(a+b)(2 \delta c-\delta f)-2(3 \delta c-4 \delta f))}{9}\right\}\right]\right] \\
& \text { c2aR = Simplify }\left[\left\{-\frac{1}{18}(a-b) t(3(2+a+b)(3+\delta c)-2(2+3 a+3 b) \delta f),\right.\right. \\
& \left.\left.\frac{(b-a) t(3(a+b)(2 \delta c-\delta f)+2(9-\delta f))}{9}\right\}\right] \\
& \left\{\frac{1}{18}(a-b) t(-9(a+b)+(-16+3 a+3 b) \delta),-\frac{1}{9}(a-b)(2+3 a+3 b) t \delta\right\} \\
& \left\{\frac{1}{18}(a-b) t(3 a(-3+\delta)+3 b(-3+\delta)-2(9+\delta)), \frac{1}{9}(-a+b) t(18+(-2+3 a+3 b) \delta)\right\}
\end{aligned}
$$

As shown above, the left endpoint of Firm A's reaction function in (Case ii) coincides with the right endpoint of Firm A's reaction function in (Case i). That is, Firm A's reaction function is continuous in (Case i) and (Case ii).

(Case iii): The first endpoint below locates the left-hand side of Firm A's reaction function, and the second endpoint below locates the right-hand side of Firm A's reaction function in period 1. 


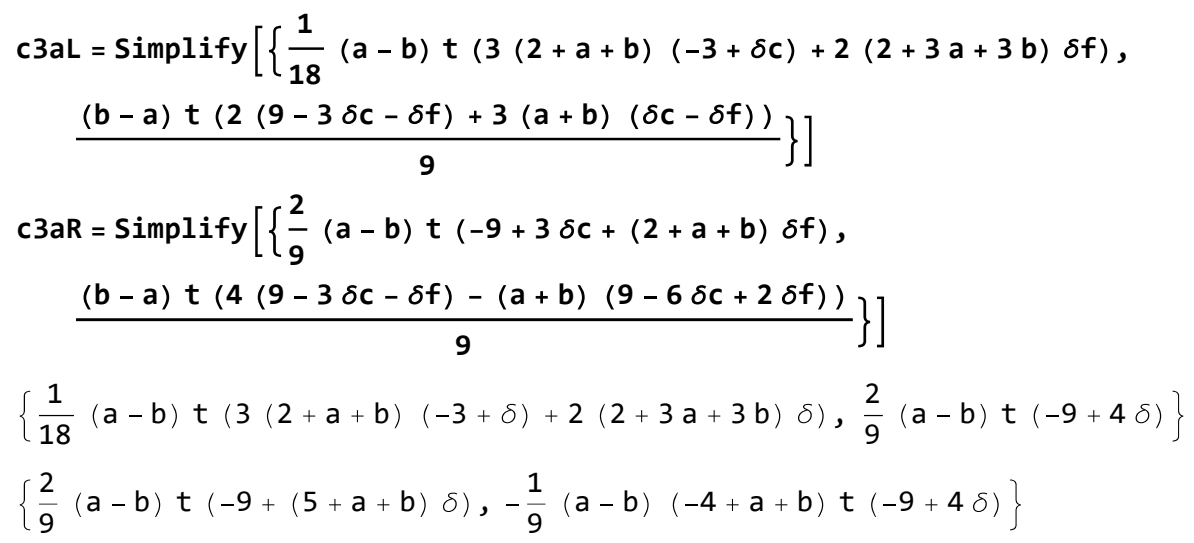

At this stage, we cannot say if Firm A's reaction function is also continuous in (Case ii) and (Case iii). We will come back to this shortly. Note that the left endpoint in (Case iii) corresponds to $(2+a+b) / 4=z$.

(Case iii)': When $\mathrm{z}=1$, the reaction function of Firm A consists of the segment connecting the following two points.

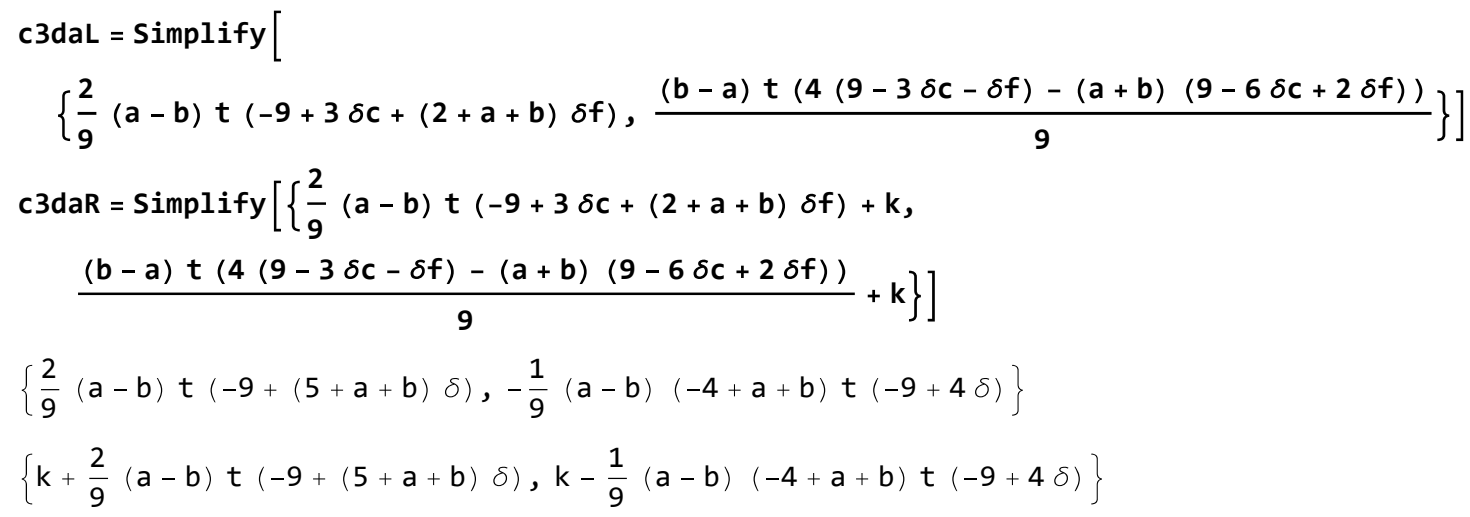

where $k$ is a sufficient large positive number (to keep $p_{A}$ at the monopoly price leading to $z=1$ ).

Next, from Firm B's reaction function derived above, we check the endpoints of each of the three line segments corresponding to the three cases: (i) $0 \leq \mathrm{z} \leq(\mathrm{a}+\mathrm{b}) / 4$, (ii) $(\mathrm{a}+\mathrm{b}) / 4<\mathrm{z}<(2+\mathrm{a}+\mathrm{b}) / 4$, (iii) $(2+\mathrm{a}+\mathrm{b}) / 4 \leq \mathrm{z} \leq 1$.

(Case i): The first endpoint below locates the left-hand side of Firm B's reaction function, and the second endpoint below locates the right-hand side of Firm B's reaction function in period 1. 


$$
\begin{aligned}
& \text { c1bL }=\text { Simplify }\left[\left\{\frac{(b-a) t(18-3(a+b)(\delta c-\delta f)-8 \delta f)}{9},\right.\right. \\
& \left.\left.\quad-\frac{1}{18}(a-b) t(3(-4+a+b)(-3+\delta c)+2(-8+3 a+3 b) \delta f)\right\}\right] \\
& \text { c1bR }=\text { Simplify }\left[\left\{\frac{(b-a) t(18+(a+b)(9-6 \delta c+2 \delta f)-8 \delta f)}{9},\right.\right. \\
& \left.\left.\quad-\frac{2}{9}(a-b) t(9-3 \delta c+(-4+a+b) \delta f)\right\}\right] \\
& \left\{\frac{2}{9}(a-b) t(-9+4 \delta),-\frac{1}{18}(a-b) t(36+9 a(-1+\delta)+9 b(-1+\delta)-28 \delta)\right\} \\
& \left\{\frac{1}{9}(a-b)(2+a+b) t(-9+4 \delta),-\frac{2}{9}(a-b) t(9+(-7+a+b) \delta)\right\}
\end{aligned}
$$

(Case i)': When $z=0$, The reaction function of Firm B consists of the segment connecting the following two points.

$$
\begin{aligned}
& \text { c1dbL }= \\
& \text { Simplify }\left[\left\{\frac{(b-a) t(18+(a+b)(9-6 \delta c+2 \delta f)-8 \delta f)}{9},-\frac{2}{9}(a-b) t(9-3 \delta c+(-4+a+b) \delta f)\right\}\right] \\
& \text { c1dbR = Simplify }\left[\left\{\frac{(b-a) t(18+(a+b)(9-6 \delta c+2 \delta f)-8 \delta f)}{9}+k,\right.\right. \\
& \left.\left.\quad-\frac{2}{9}(a-b) t(9-3 \delta c+(-4+a+b) \delta f)+k\right\}\right] \\
& \left\{\frac{1}{9}(a-b)(2+a+b) t(-9+4 \delta),-\frac{2}{9}(a-b) t(9+(-7+a+b) \delta)\right\} \\
& \left\{k+\frac{1}{9}(a-b)(2+a+b) t(-9+4 \delta), k-\frac{2}{9}(a-b) t(9+(-7+a+b) \delta)\right\}
\end{aligned}
$$

where $k$ is a sufficient large positive number (to keep $p_{B}$ at the monopoly price leading to $z=0$ ).

Case (ii): The first endpoint below locates the left-hand side of Firm B's reaction function, and the second endpoint below locates the right-hand side of Firm B's reaction function in period 1.

$$
\begin{aligned}
& \text { c2bL }=\text { Fullsimplify }\left[\left\{\frac{(b-a) t(6(1-a-b) \delta c+(2+3 a+3 b) \delta f)}{9},\right.\right. \\
& \left.\left.\quad \frac{1}{18}(a-b) t(3(-2+a+b)(3+\delta c)-2(2+3 a+3 b) \delta f)\right\}\right] \\
& \text { c2bR }=\text { Simplify }\left[\left\{\frac{(b-a) t(18+6(2-a-b) \delta c-(8-3 a-3 b) \delta f)}{9},\right.\right. \\
& \left.\left.\quad \frac{1}{18}(a-b) t(3(-4+a+b)(3+\delta c)-2(-8+3 a+3 b) \delta f)\right\}\right] \\
& \left\{\frac{1}{9}(a-b)(-8+3 a+3 b) t \delta,-\frac{1}{18}(a-b) t(-9(-2+a+b)+(10+3 a+3 b) \delta)\right\} \\
& \left\{\frac{1}{9}(a-b) t(-18+(-4+3 a+3 b) \delta),-\frac{1}{18}(a-b) t(-4(-9+\delta)+3 a(-3+\delta)+3 b(-3+\delta))\right\}
\end{aligned}
$$

At this stage, we cannot say if Firm B's reaction function is continuous in (Case i) and (Case ii). We will come back to this shortly. Note that the left-hand endpoint in (Case i) corresponds to $(\mathrm{a}+\mathrm{b}) / 4=\mathrm{z}$.

(Case iii): The first endpoint below locates the left-hand side of Firm B's reaction function, and the second endpoint below locates the right-hand side of Firm B's reaction function in period 1. 


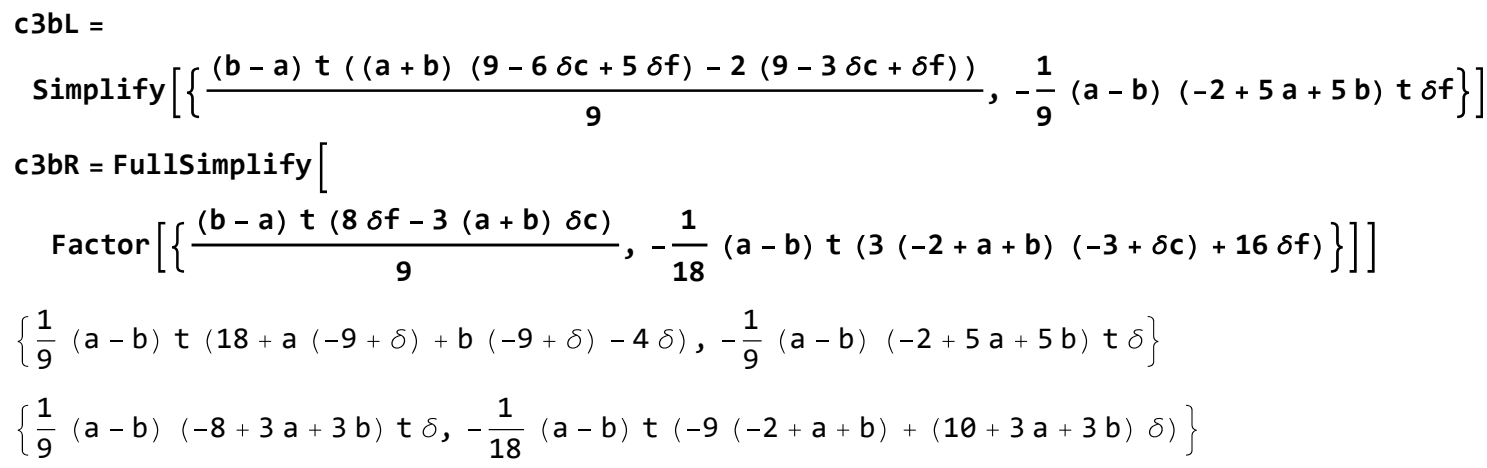

We find that the left endpoint of Firm B's reaction function in (Case ii) coincides with the right endpoint of Firm B's reaction function in (Case iii). That is, Firm B's reaction function is continuous in (Case ii) and (Case iii).

The exact shape of the above reaction functions depends on various parameters of the model and the values of $(a, b)$ that are given at this stage. In what follows, we show first that, for any values of $(a, b)$, each firm's reaction function has one discontinuity point. Second, we show that the two reaction functions intersect only in (Case ii) given the restrictions on $\mathrm{a}+\mathrm{b}$ and $\delta$ stated in Proposition 1. This shows that the equilibrium is possible only in (Case ii). We then show that the unique location equilibrium leading to the pricing equilibrium corresponding to (Case ii) is given by $a=0, b=1$.

We start by deriving Firm A's 'true' reaction function illustrating the discontinuity using various examples. We repeat the same for Firm B's 'true' reaction function. Calculations in the two parts are quite messy. If necessary, readers can refer to various figures provided, and skip the calculations to jump directly to the third part, where we show the pricing equilibrium in the first period is possible only in (Case ii).

For illustrative purposes, we start with an example where we set $\mathrm{a}=0, \mathrm{~b}=1, \mathrm{t}=1$, and $\delta=1 / 2$.

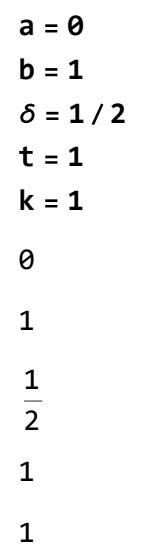

First, we plot Firm A's reaction function corresponding to the three cases. 


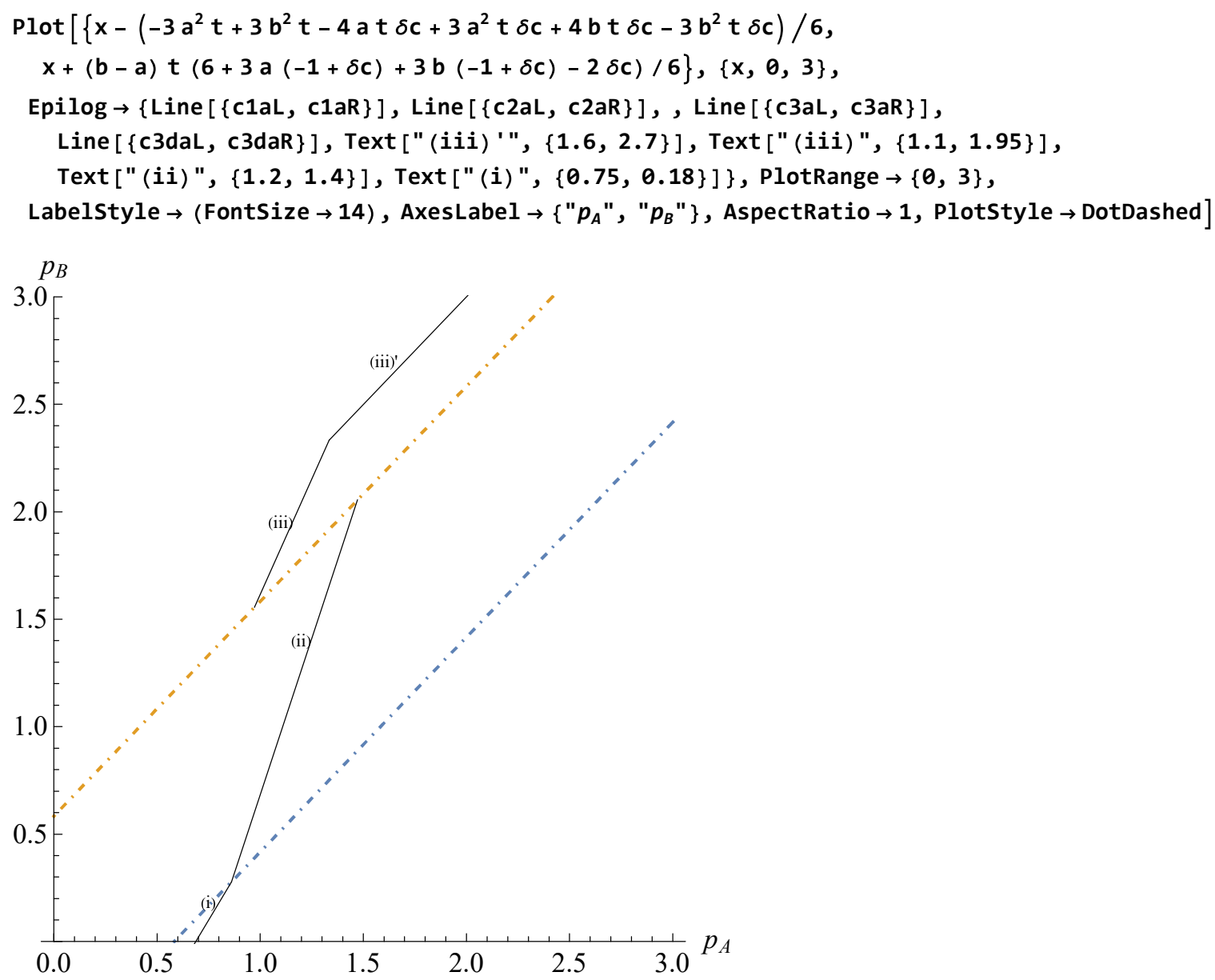

As shown above, for some $p_{B}$, there are two local optimal prices for Firm A.

We can show that the multiplicity of local optimal prices always appears.

Clear $[a, b, \delta, t, k]$

To show the multiplicity, we check the locations of the three endpoints: The right-hand endpoint in (ii) (c2aR), the left-hand and right-hand endpoints (c3aL and c3aR) in (iii) (see below)

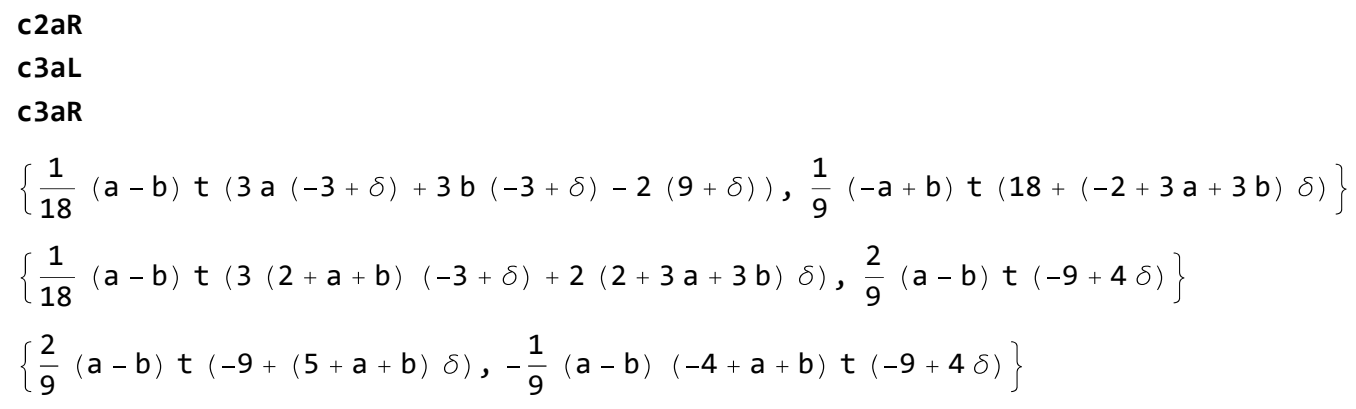

First, we compare the elements of the right-hand endpoint in (ii) and the left-hand endpoint in (iii): 


$$
\begin{aligned}
& \text { Factor }\left[\frac{1}{18}(a-b) t(3 a(-3+\delta)+3 b(-3+\delta)-2(9+\delta))-\right. \\
& \left.\quad \frac{1}{18}(a-b) \text { t }(3(2+a+b)(-3+\delta)+2(2+3 a+3 b) \delta)\right] \\
& \text { Factor }\left[\frac{1}{9}(-a+b) t(18+(-2+3 a+3 b) \delta)-\frac{2}{9}(a-b) t(-9+4 \delta)\right] \\
& -\frac{1}{3}(a-b)(2+a+b) \text { t } \delta \\
& -\frac{1}{3}(a-b)(2+a+b) t \delta
\end{aligned}
$$

The outcome means that for any $\mathrm{a} \in[0,1], \mathrm{b} \in[0,1](\mathrm{a} \geq \mathrm{b}), \mathrm{t}$, and $\delta$, the right-hand endpoint in (ii) is located above the left-hand endpoint in (iii) as in the above Figure.

Second, we compare the $p_{B}$-elements of the right-hand endpoint in (ii) and the right-hand endpoint in (iii):

Factor $\left[\frac{1}{9}(-a+b) t(18+(-2+3 a+3 b) \delta)-\left(-\frac{1}{9}(a-b)(-4+a+b) t(-9+4 \delta)\right)\right]$

$\frac{1}{9}(a-b) t(18-9 a-9 b-14 \delta+a \delta+b \delta)$

The $p_{B}$-element of the right-hand endpoint in (ii) larger than that of the right-hand endpoint in (iii) if and only if

$\frac{2(9-7 \delta)}{9-\delta}<a+b$

If $\frac{2(9-7 \delta)}{9-\delta} \geq a+b$, we simply compare the reaction function in (ii) and the reaction function in (iii); If $\frac{2(9-7 \delta)}{9-\delta}<a+b$, in addition to the previous comparison, we also compare the reaction function in (ii) and the reaction function in (iii)'.

Plot3D $\left[\mathrm{g}-\frac{2(9-7 \delta)}{9-\delta},\{\delta, 0,1\},\{\mathrm{g}, 0,2\}\right.$, PlotRange $\left.\rightarrow\{0,1.5\}\right]$

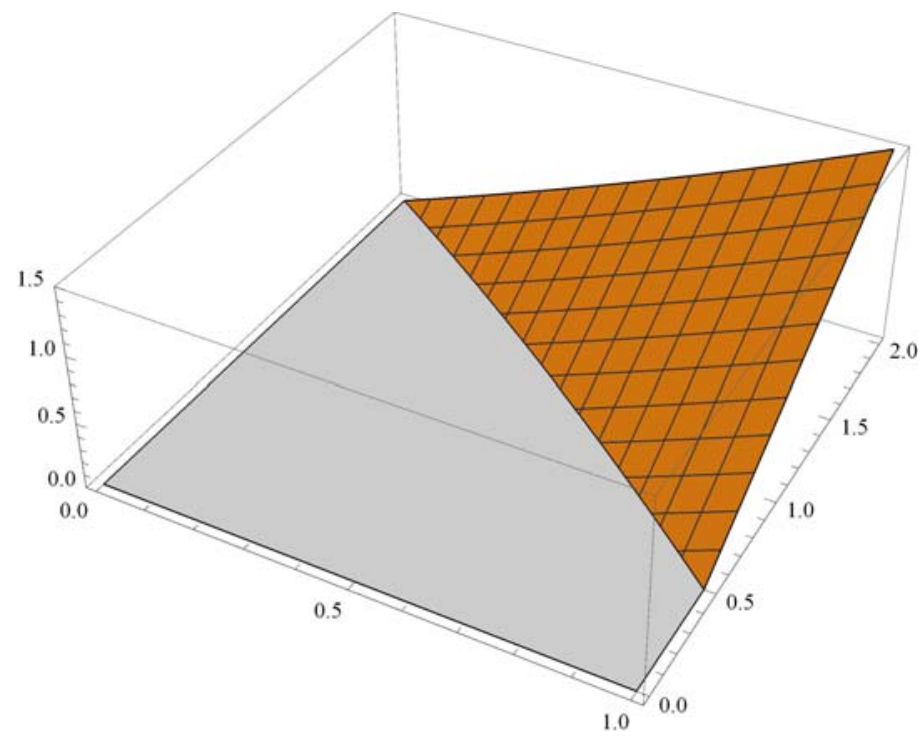

We need to find the global optimal price of Firm A, $p_{A}$, when there are two local optima for a given $p_{B}$. There is a price $p_{B}$ such that choosing the reaction function in (ii) and choosing the reaction function in (iii) or the reaction function in (iii)' are indifferent for Firm $\mathrm{A}$. This $p_{B}$ is the threshold for which choosing the reaction function in (ii) is preferred by Firm $\mathrm{A}$ if $p_{B}$ is 
smaller than this threshold; otherwise, choosing the reaction function in (iii) is preferred by Firm A. We need to find the threshold value of $p_{B}$.

To check the threshold value of $p_{B}$ for Firm A's reaction function, we derive the profits under cases (ii), (iii), and (iii)'.

The interior profit of firm $\mathrm{A}$ under case (ii) for $p_{B}$ is

$$
\begin{aligned}
& \text { Factor }\left[p_{A} z+\delta f \frac{1}{9}(b-a) t\left(2+2 a+a^{2}+2 b+2 a b+b^{2}-8 z-2 a z-2 b z+10 z^{2}\right) / .\right. \\
& z \rightarrow \frac{3\left(p_{B}-p_{A}\right)}{2(b-a) t(3+\delta c)}+\frac{((a+b)(3-\delta c)+2 \delta c)}{2(3+\delta c)} / \cdot\left\{p_{A} \rightarrow\right. \\
& \left((9+3 \delta c-10 \delta f) p_{B}+(b-a) t\left((a+b)\left(9-\delta c^{2}-8 \delta f+4 \delta c \delta f\right)+2\left(3 \delta c+\delta c^{2}+4 \delta f-2 \delta c \delta f\right)\right)\right) / \\
& (2(9+3 \delta c-5 \delta f))\}] \\
& \frac{1}{72(a-b) t(-9+2 \delta)} \\
& \left(81 a^{4} t^{2}-162 a^{2} b^{2} t^{2}+81 b^{4} t^{2}+144 a^{2} t^{2} \delta+108 a^{3} t^{2} \delta-18 a^{4} t^{2} \delta-288 a b t^{2} \delta-108 a^{2} b t^{2} \delta+\right. \\
& 144 b^{2} t^{2} \delta-108 a b^{2} t^{2} \delta+36 a^{2} b^{2} t^{2} \delta+108 b^{3} t^{2} \delta-18 b^{4} t^{2} \delta-28 a^{2} t^{2} \delta^{2}-12 a^{3} t^{2} \delta^{2}+ \\
& 9 a^{4} t^{2} \delta^{2}+56 a b t^{2} \delta^{2}+12 a^{2} b t^{2} \delta^{2}-28 b^{2} t^{2} \delta^{2}+12 a b^{2} t^{2} \delta^{2}-18 a^{2} b^{2} t^{2} \delta^{2}-12 b^{3} t^{2} \delta^{2}+ \\
& \left.9 b^{4} t^{2} \delta^{2}-162 a^{2} t p_{B}+162 b^{2} t p_{B}+36 a t \delta p_{B}+90 a^{2} t \delta p_{B}-36 b t \delta p_{B}-90 b^{2} t \delta p_{B}+81 p_{B}^{2}\right)
\end{aligned}
$$

The interior profit of firm $\mathrm{A}$ under case (iii) for $p_{B}$

$$
\begin{aligned}
& \text { Factor }\left[p_{A} z+\delta f\left(\frac{1}{18}(b-a) t(a+b+2 z)^{2}\right) /, z \rightarrow \frac{-3 p_{A}+3 p_{B}-3 a^{2} t+3 b^{2} t+2 a^{2} t \delta c-2 b^{2} t \delta c}{2(a-b) t(-3+\delta c)} / .\right. \\
& \qquad \begin{array}{l}
\left.p_{A} \rightarrow \frac{(9-3 \delta c-2 \delta f) p_{B}}{2(9-3 \delta c-\delta f)}+\frac{(b-a)(a+b) t((3-\delta c)(3-2 \delta c)-2(2-\delta c) \delta f)}{2(9-3 \delta c-\delta f)}\right] \\
-\left(\left(-9 a^{4} t^{2}+18 a^{2} b^{2} t^{2}-9 b^{4} t^{2}+4 a^{4} t^{2} \delta-8 a^{2} b^{2} t^{2} \delta+4 b^{4} t^{2} \delta+\right.\right. \\
\left.\left.\quad 18 a^{2} t p_{B}-18 b^{2} t p_{B}-8 a^{2} t \delta p_{B}+8 b^{2} t \delta p_{B}-9 p_{B}^{2}\right) /(8(a-b) t(-9+4 \delta))\right)
\end{array}
\end{aligned}
$$

We derive the threshold value of $p_{B}$ by finding $p_{B}$ that equalizes the above two profits:

$$
\begin{aligned}
& \text { Fullsimplify [ } \\
& \text { Solve }\left[\left\{\frac { 1 } { 7 2 ( a - b ) t ( - 9 + 2 \delta ) } \left(81 a^{4} t^{2}-162 a^{2} b^{2} t^{2}+81 b^{4} t^{2}+144 a^{2} t^{2} \delta+108 a^{3} t^{2} \delta-18 a^{4} t^{2} \delta-\right.\right.\right. \\
& 288 a b t^{2} \delta-108 a^{2} b t^{2} \delta+144 b^{2} t^{2} \delta-108 a b^{2} t^{2} \delta+36 a^{2} b^{2} t^{2} \delta+108 b^{3} t^{2} \delta- \\
& 18 b^{4} t^{2} \delta-28 a^{2} t^{2} \delta^{2}-12 a^{3} t^{2} \delta^{2}+9 a^{4} t^{2} \delta^{2}+56 a b t^{2} \delta^{2}+12 a^{2} b t^{2} \delta^{2}- \\
& 28 b^{2} t^{2} \delta^{2}+12 a b^{2} t^{2} \delta^{2}-18 a^{2} b^{2} t^{2} \delta^{2}-12 b^{3} t^{2} \delta^{2}+9 b^{4} t^{2} \delta^{2}-162 a^{2} t p_{B}+ \\
& \left.162 b^{2} t p_{B}+36 a t \delta p_{B}+90 a^{2} t \delta p_{B}-36 b t \delta p_{B}-90 b^{2} t \delta p_{B}+81 p_{B}^{2}\right)== \\
& -\left(\left(-9 a^{4} t^{2}+18 a^{2} b^{2} t^{2}-9 b^{4} t^{2}+4 a^{4} t^{2} \delta-8 a^{2} b^{2} t^{2} \delta+4 b^{4} t^{2} \delta+18 a^{2} t p_{B}-\right.\right. \\
& \left.\left.\left.\left.\left.18 b^{2} t p_{B}-8 a^{2} t \delta p_{B}+8 b^{2} t \delta p_{B}-9 p_{B}^{2}\right) /(8(a-b) t(-9+4 \delta))\right)\right\}, p_{B}\right]\right] \\
& \left\{\left\{p_{B} \rightarrow-\frac{1}{18 \delta} t(-9+4 \delta)\left(2 a \delta+3 a^{2} \delta-2 b \delta-3 b^{2} \delta+3(-9+2 \delta) \sqrt{\frac{(a-b)^{2}(2+a+b)^{2} \delta^{2}}{(-9+2 \delta)(-9+4 \delta)}}\right)\right\},\right. \\
& \left.\left\{p_{B} \rightarrow \frac{1}{18 \delta} t(-9+4 \delta)\left(-2 a \delta-3 a^{2} \delta+2 b \delta+3 b^{2} \delta+3(-9+2 \delta) \sqrt{\frac{(a-b)^{2}(2+a+b)^{2} \delta^{2}}{(-9+2 \delta)(-9+4 \delta)}}\right)\right\}\right\}
\end{aligned}
$$

We can easily show that the former outcome is negative. So, we use the latter one.

We simplify the expression of the latter outcome, and obtain the following $p_{B}$ : 
$p_{B} \rightarrow \frac{t(9-4 \delta)(b-a)\left(3(2+a+b) \sqrt{\frac{9-2 \delta}{9-4 \delta}}-(2+3 a+3 b)\right)}{18}$ (pb1)

We rewrite the locations of the two endpoints: The right-hand endpoint in (ii), the right-hand endpoints in (iii) (see below)

c2aR

C3aR

$\left\{\frac{1}{18}(a-b) t(3 a(-3+\delta)+3 b(-3+\delta)-2(9+\delta)), \frac{1}{9}(-a+b) t(18+(-2+3 a+3 b) \delta)\right\}$

$\left\{\frac{2}{9}(a-b) t(-9+(5+a+b) \delta),-\frac{1}{9}(a-b)(-4+a+b) t(-9+4 \delta)\right\}$

We check the condition that the derived $p_{B}(\mathrm{pb} 1)$ is below the $p_{B}$-element of the right-hand endpoints in (iii).

Simplify [Factor [

$$
\begin{aligned}
& \left.\left.-\frac{1}{9}(a-b)(-4+a+b) t(-9+4 \delta)-\frac{t(9-4 \delta)(b-a)\left(3(2+a+b) \sqrt{\frac{9-2 \delta}{9-4 \delta}}-(2+3 a+3 b)\right.}{18}\right]\right] \\
& -\frac{1}{18}(a-b) t\left(-10+a\left(-1+3 \sqrt{\frac{9-2 \delta}{9-4 \delta}}\right)+b\left(-1+3 \sqrt{\frac{9-2 \delta}{9-4 \delta}}\right)+6 \sqrt{\frac{9-2 \delta}{9-4 \delta}}\right)(-9+4 \delta)
\end{aligned}
$$

This is positive if and only if the following inequality holds

$$
\begin{aligned}
& (a+b)\left(-1+3 \sqrt{\frac{9-2 \delta}{9-4 \delta}}\right)<10-6 \sqrt{\frac{9-2 \delta}{9-4 \delta}} \\
& \text { Plot }\left[\left(10-6 \sqrt{\frac{9-2 \delta}{9-4 \delta}}\right) /\left(-1+3 \sqrt{\frac{9-2 \delta}{9-4 \delta}}\right),\{\delta, 0,1\}\right]
\end{aligned}
$$

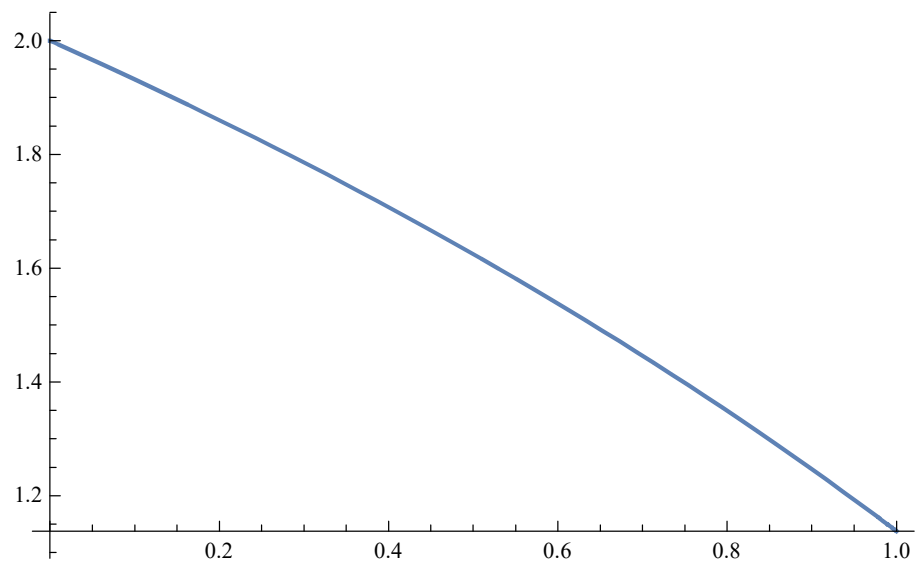

If $(\mathrm{a}+\mathrm{b})\left(-1+3 \sqrt{\frac{9-2 \delta}{9-4 \delta}}\right)<10-6 \sqrt{\frac{9-2 \delta}{9-4 \delta}}$ holds, the derived $p_{B}(\mathrm{pb} 1)$ is on the interval between the left-hand and right-hand endpoints in (iii). Otherwise, the reaction function in (ii) is always better than the interval between the left-hand and right-hand endpoints in (iii).

Now we derive the profit of firm A under case (iii)' for $p_{B}$ 


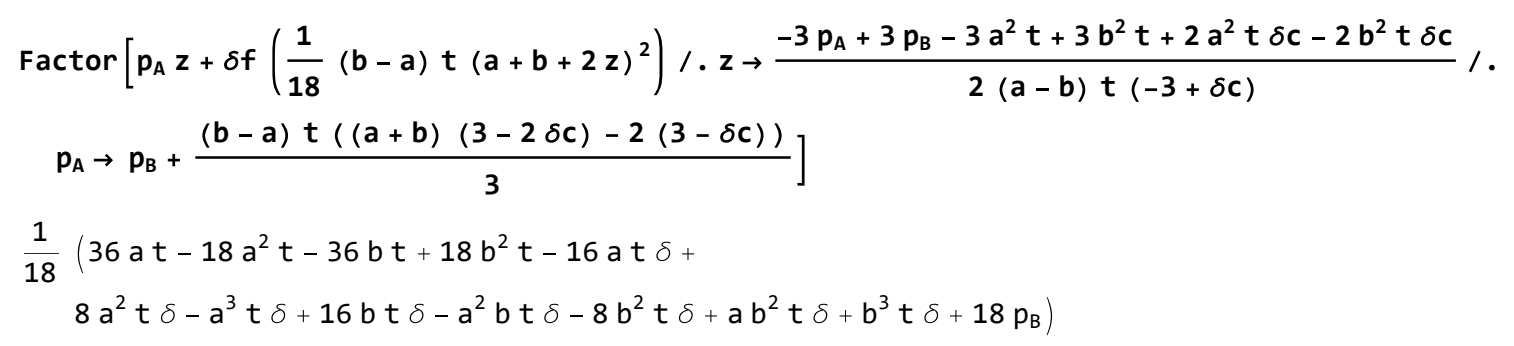

We derive the threshold value of $p_{B}$ by finding $p_{B}$ that equalizes the two profits in cases (ii) and (iii)':

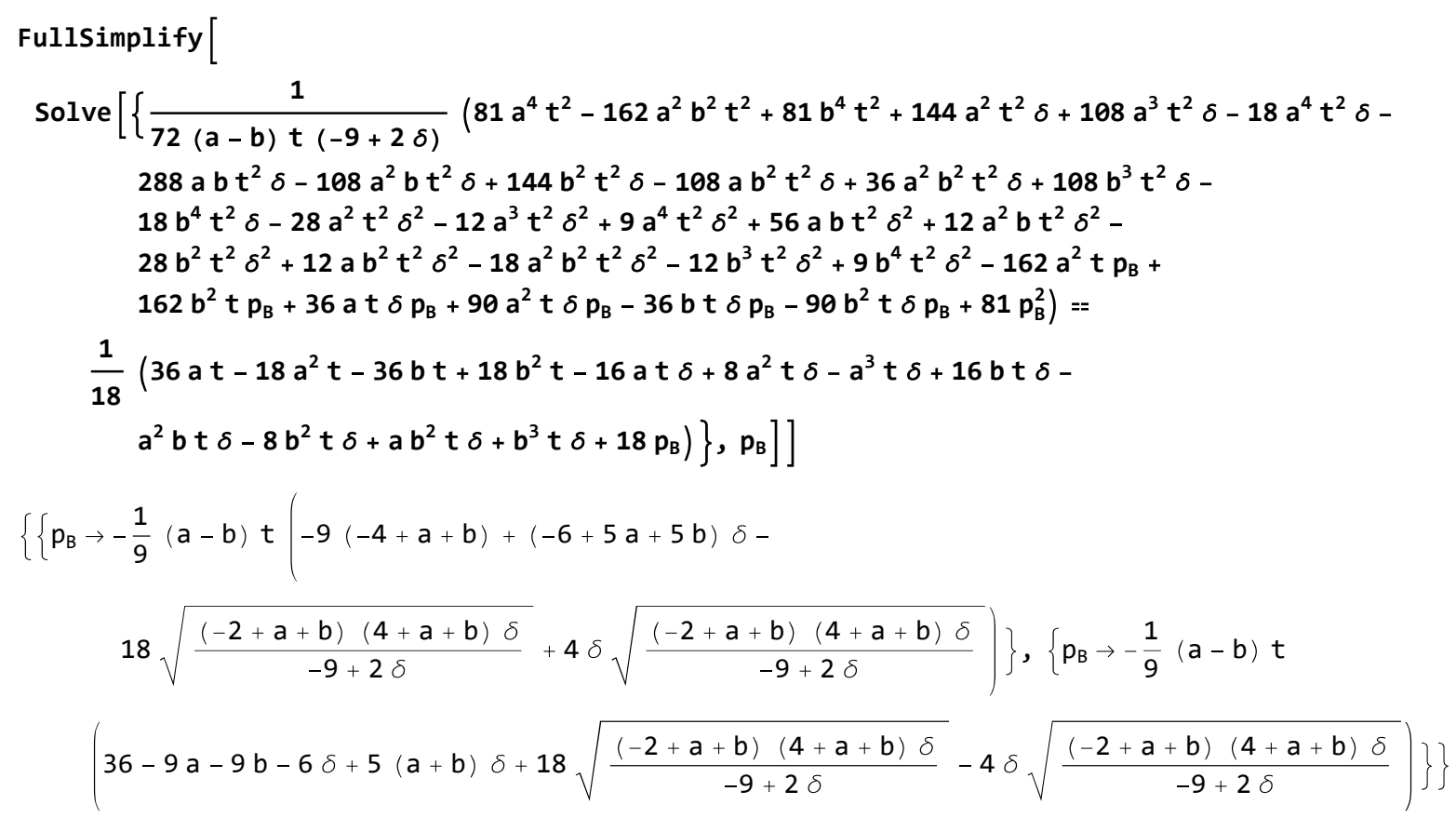

We pick up the first outcome as the threshold $p_{B}$.

$$
\begin{aligned}
p_{B} \rightarrow-\frac{1}{9}(a-b) t(-9(-4+a+b)+(-6+5 a+5 b) \delta- \\
\left.18 \sqrt{\frac{(-2+a+b)(4+a+b) \delta}{-9+2 \delta}}+4 \delta \sqrt{\frac{(-2+a+b)(4+a+b) \delta}{-9+2 \delta}}\right) \quad(p b 2)
\end{aligned}
$$

We check the condition that the derived $p_{B}(\mathrm{pb} 2)$ is above the $p_{B}$-element of the right-hand endpoints in (iii).

$$
\begin{aligned}
& \text { Simplify }\left[\text { Factor } \left[-\frac{1}{9}(a-b) t\left(-9(-4+a+b)+(-6+5 a+5 b) \delta-18 \sqrt{\frac{(-2+a+b)(4+a+b) \delta}{-9+2 \delta}}+\right.\right.\right. \\
& \left.\left.\left.\quad 4 \delta \sqrt{\frac{(-2+a+b)(4+a+b) \delta}{-9+2 \delta}}\right)-\left(-\frac{1}{9}(a-b)(-4+a+b) t(-9+4 \delta)\right)\right]\right] \\
& -\frac{1}{9}(a-b) t\left(10 \delta+a \delta+b \delta-18 \sqrt{\frac{(-2+a+b)(4+a+b) \delta}{-9+2 \delta}}+4 \delta \sqrt{\frac{(-2+a+b)(4+a+b) \delta}{-9+2 \delta}}\right)
\end{aligned}
$$

Denoting $a+b$ by $g$, we check the value between the largest parentheses: 

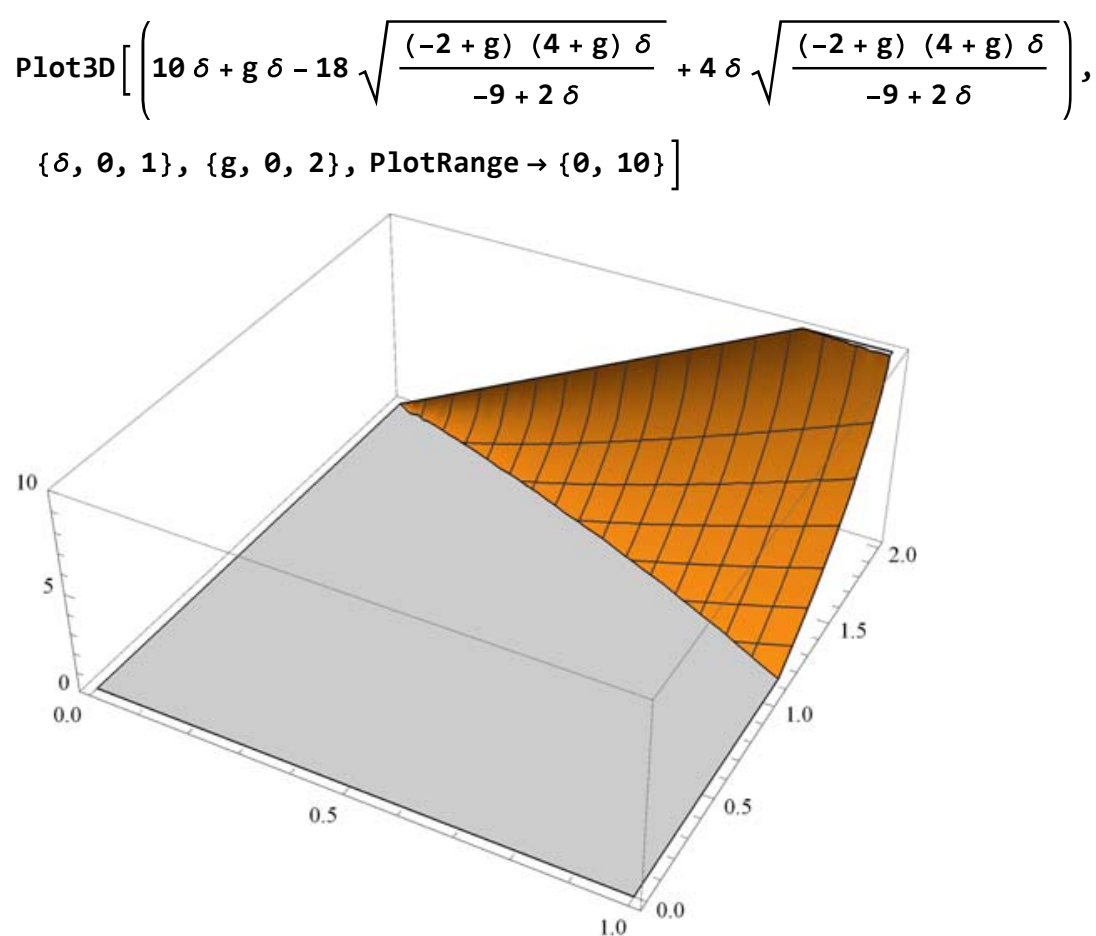

We check the condition that the value between the large parentheses is positive:

$$
\begin{aligned}
& \text { Solve }\left[\left(10 \delta+\mathrm{g} \delta-18 \sqrt{\frac{(-2+\mathrm{g})(4+\mathrm{g}) \delta}{-9+2 \delta}}+4 \delta \sqrt{\frac{(-2+\mathrm{g})(4+\mathrm{g}) \delta}{-9+2 \delta}}\right)=0, \mathrm{~g}\right] \\
& \left\{\left\{\mathrm{g} \rightarrow \frac{2\left(18+\delta-6 \sqrt{81-54 \delta+8 \delta^{2}}\right)}{-36+7 \delta}\right\},\left\{\mathrm{g} \rightarrow \frac{2\left(18+\delta+6 \sqrt{81-54 \delta+8 \delta^{2}}\right)}{-36+7 \delta}\right\}\right\} \\
& \text { If } \mathrm{g}=\mathrm{a}+\mathrm{b}>\frac{2\left(18+\delta-6 \sqrt{81-54 \delta+8 \delta^{2}}\right)}{-36+7 \delta},
\end{aligned}
$$

the derived $p_{B}(\mathrm{pb} 2)$ is above the $p_{B}$-element of the right-hand endpoints in (iii).

This coincides with the following condition that the derived $p_{B}(\mathrm{pb} 1)$ is above the $p_{B}$-element of the right-hand endpoints in (iii).
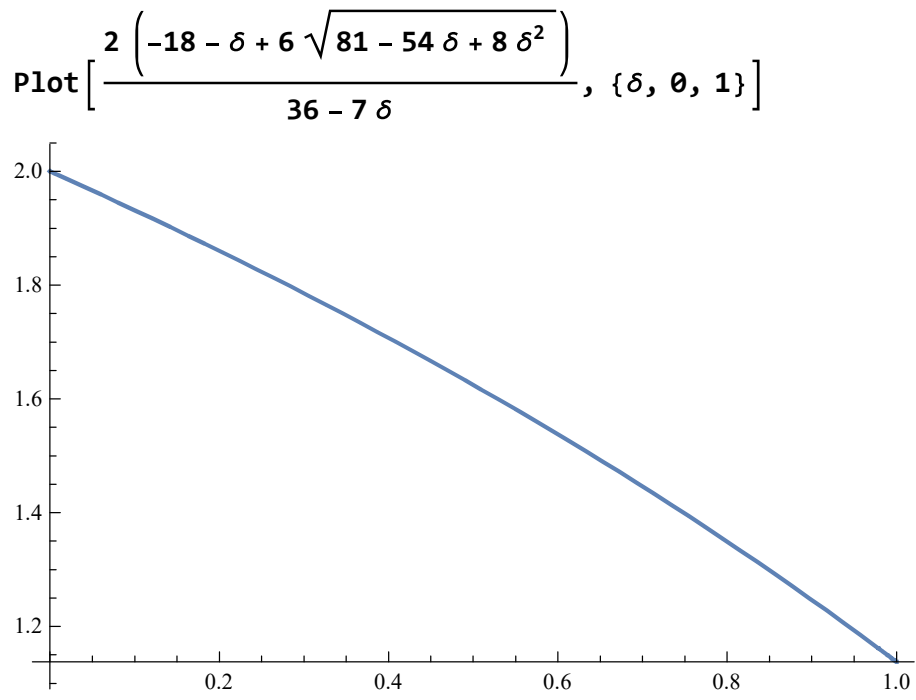

If $(\mathrm{a}+\mathrm{b})<\frac{2\left(-18-\delta+6 \sqrt{81-54 \delta+8 \delta^{2}}\right)}{36-7 \delta}$ holds, the threshold $p_{B}$ is on the line segment between the 
left-hand and right-hand endpoints in (iii). The threshold is given as

$$
p_{B} \rightarrow \frac{t(9-4 \delta)(b-a)\left(3(2+a+b) \sqrt{\frac{9-2 \delta}{9-4 \delta}}-(2+3 a+3 b)\right)}{18} \text { (pb1) }
$$

For the threshold value of $p_{B}(\mathrm{pb} 1)$, the point of $p_{A}$-element in Firm A's reaction function in (ii) is

$$
\begin{aligned}
& \text { Simplify }\left[\text { Expand } \left[p_{A} \rightarrow\right.\right. \\
& \left((9+3 \delta c-10 \delta f) p_{B}+(b-a) t\left((a+b)\left(9-\delta c^{2}-8 \delta f+4 \delta c \delta f\right)+2\left(3 \delta c+\delta c^{2}+4 \delta f-2 \delta c \delta f\right)\right)\right) / \\
& \left.\left.(2(9+3 \delta c-5 \delta f)) / . p_{B} \rightarrow \frac{t(9-4 \delta)(b-a)\left(3(2+a+b) \sqrt{\frac{9-2 \delta}{9-4 \delta}}-(2+3 a+3 b)\right)}{18}\right]\right] \\
& \mathrm{p}_{\mathrm{A}} \rightarrow \frac{1}{36(-9+2 \delta)}(\mathrm{a}-\mathrm{b}) \mathrm{t}\left(3 \mathrm{a}\left(-27+81 \sqrt{\frac{9-2 \delta}{9-4 \delta}}+\left(51-99 \sqrt{\frac{9-2 \delta}{9-4 \delta}}\right) \delta+2\left(-5+14 \sqrt{\frac{9-2 \delta}{9-4 \delta}}\right) \delta^{2}\right)+\right. \\
& 3 b\left(-27+81 \sqrt{\frac{9-2 \delta}{9-4 \delta}}+\left(51-99 \sqrt{\frac{9-2 \delta}{9-4 \delta}}\right) \delta+2\left(-5+14 \sqrt{\frac{9-2 \delta}{9-4 \delta}}\right) \delta^{2}\right)+ \\
& \left.2\left(81\left(-1+3 \sqrt{\frac{9-2 \delta}{9-4 \delta}}\right)-9\left(-25+33 \sqrt{\frac{9-2 \delta}{9-4 \delta}}\right) \delta+\left(-46+84 \sqrt{\frac{9-2 \delta}{9-4 \delta}}\right) \delta^{2}\right)\right)
\end{aligned}
$$

We simplify the above outcome, and obtain the following:

$$
\begin{aligned}
& \mathrm{p}_{\mathrm{A}} \rightarrow \frac{1}{36(9-2 \delta)}(\mathrm{b}-\mathrm{a}) \mathrm{t} \\
& \left((9-2 \delta)(-18+46 \delta+3(\mathrm{a}+\mathrm{b})(-3+5 \delta))+3(2+a+b) \sqrt{\frac{9-2 \delta}{9-4 \delta}}\left(81-99 \delta+28 \delta^{2}\right)\right)
\end{aligned}
$$

We can define the jumping point of Firm A's reaction function in (ii) as c2ja1

$$
\begin{aligned}
& \text { c2ja1 }=\left\{\frac{1}{36(9-2 \delta)}(b-a) t\right. \\
& \left.\quad(9-2 \delta)(-18+46 \delta+3(a+b)(-3+5 \delta))+3(2+a+b) \sqrt{\frac{9-2 \delta}{9-4 \delta}}\left(81-99 \delta+28 \delta^{2}\right)\right), \\
& \quad t(9-4 \delta)(b-a)\left(3(2+a+b) \sqrt{\frac{9-2 \delta}{9-4 \delta}}-(2+3 a+3 b)\right) \\
& \left.\quad \frac{18}{36(9-2 \delta)}\right\} \\
& \quad(-a+b) t\left(3(2+a+b) \sqrt{\frac{9-2 \delta}{9-4 \delta}}\left(81-99 \delta+28 \delta^{2}\right)+(9-2 \delta)(-18+46 \delta+3(a+b)(-3+5 \delta))\right) \\
& \left.\frac{1}{18}(-a+b) t\left(-2-3 a-3 b+3(2+a+b) \sqrt{\frac{9-2 \delta}{9-4 \delta}}\right)(9-4 \delta)\right\}
\end{aligned}
$$

Also, for the threshold value of $p_{B}$ (pb1), the point of $p_{A}$-element in Firm A's reaction function in (iii) is 


$$
\begin{aligned}
& \text { Simplify }\left[\text { Expand } \left[p_{A} \rightarrow \frac{(9-3 \delta c-2 \delta f) p_{B}}{2(9-3 \delta c-\delta f)}+\frac{(b-a)(a+b) t((3-\delta c)(3-2 \delta c)-2(2-\delta c) \delta f)}{2(9-3 \delta c-\delta f)} / .\right.\right. \\
& \quad t(9-4 \delta)(b-a)\left(3(2+a+b) \sqrt{\frac{9-2 \delta}{9-4 \delta}}-(2+3 a+3 b)\right] \\
& p_{B} \rightarrow \frac{18}{p_{A} \rightarrow \frac{1}{36}(a-b) t\left(2\left(-1+3 \sqrt{\frac{9-2 \delta}{9-4 \delta}}\right)(-9+5 \delta)+\right.} \\
& \left.\quad 3 a\left(3-9 \sqrt{\frac{9-2 \delta}{9-4 \delta}}+\delta+5 \sqrt{\frac{9-2 \delta}{9-4 \delta}} \delta\right)+3 b\left(3-9 \sqrt{\frac{9-2 \delta}{9-4 \delta}}+\delta+5 \sqrt{\frac{9-2 \delta}{9-4 \delta}} \delta\right)\right)
\end{aligned}
$$

We simplify the above outcome, and obtain the following:

$$
p_{A} \rightarrow \frac{1}{36}(b-a) t\left(-2(9-5 \delta)-3(a+b)(3+\delta)+3(9-5 \delta)(2+a+b) \sqrt{\frac{9-2 \delta}{9-4 \delta}}\right)
$$

We can define the jumping point of Firm A's reaction function in (iii) as c3jal

$$
\begin{gathered}
\text { c3ja1 }=\left\{\frac{1}{36}(b-a) t\left(-2(9-5 \delta)-3(a+b)(3+\delta)+3(9-5 \delta)(2+a+b) \sqrt{\frac{9-2 \delta}{9-4 \delta}}\right),\right. \\
\left.\frac{t(9-4 \delta)(b-a)\left(3(2+a+b) \sqrt{\frac{9-2 \delta}{9-4 \delta}}-(2+3 a+3 b)\right)}{18}\right\}
\end{gathered}
$$$$
\left\{\frac{1}{36}(-a+b) t\left(-2(9-5 \delta)+3(2+a+b)(9-5 \delta) \sqrt{\frac{9-2 \delta}{9-4 \delta}}-3(a+b)(3+\delta)\right),\right.
$$$$
\left.\frac{1}{18}(-a+b) t\left(-2-3 a-3 b+3(2+a+b) \sqrt{\frac{9-2 \delta}{9-4 \delta}}\right)(9-4 \delta)\right\}
$$

If $(\mathrm{a}+\mathrm{b}) \geq \frac{2\left(-18-\delta+6 \sqrt{81-54 \delta+8 \delta^{2}}\right)}{36-7 \delta}$ holds, the threshold $p_{B}$ is on the half-line starting from the right-hand endpoints in (iii), that is, case (iii)'. The threshold is given as $\mathrm{p}_{B} \rightarrow \frac{1}{9}(\mathrm{~b}-\mathrm{a}) \mathrm{t}(9(4-\mathrm{a}-\mathrm{b})-(6-5 \mathrm{a}-5 \mathrm{~b}) \delta-2 \sqrt{(9-2 \delta)(2-a-b)(4+a+b) \delta})(\mathrm{pb2})$

For the threshold value of $p_{B}(\mathrm{pb} 2)$, the point of $p_{A}$-element in Firm A's reaction function in (ii) is

$$
\begin{aligned}
& \text { Simplify }\left[\text { Expand } \left[p_{A} \rightarrow\right.\right. \\
& \left((9+3 \delta c-10 \delta f) p_{B}+(b-a) t\left((a+b)\left(9-\delta c^{2}-8 \delta f+4 \delta c \delta f\right)+2\left(3 \delta c+\delta c^{2}+4 \delta f-2 \delta c \delta f\right)\right)\right) / \\
& (2(9+3 \delta c-5 \delta f)) / \text {. } \\
& \left.\left.p_{B} \rightarrow \frac{1}{9}(b-a) t(9(4-a-b)-(6-5 a-5 b) \delta-2 \sqrt{(9-2 \delta)(2-a-b)(4+a+b) \delta})\right]\right] \\
& \mathrm{p}_{\mathrm{A}} \rightarrow-\frac{1}{9(-9+2 \delta)}(\mathrm{a}-\mathrm{b}) \mathrm{t}\left(4(-3+\mathrm{a}+\mathrm{b}) \delta^{2}+9\left(-18+\sqrt{\left(-8+\mathrm{a}^{2}+2 \mathrm{~b}+\mathrm{b}^{2}+2 \mathrm{a}(1+\mathrm{b})\right) \delta(-9+2 \delta)}\right)-\right. \\
& \left.\delta\left(-90+18 a+18 b+7 \sqrt{\left(-8+a^{2}+2 b+b^{2}+2 a(1+b)\right) \delta(-9+2 \delta)}\right)\right)
\end{aligned}
$$

We can define the jumping point of Firm A's reaction function in (ii) as c2ja2 


$$
\begin{aligned}
& \mathrm{c} 2 \mathrm{ja2}= \\
& \left\{-\frac{1}{9(-9+2 \delta)}(a-b) t\left(4(-3+a+b) \delta^{2}+9\left(-18+\sqrt{\left(-8+a^{2}+2 b+b^{2}+2 a(1+b)\right) \delta(-9+2 \delta)}\right)-\right.\right. \\
& \left.\delta\left(-90+18 a+18 b+7 \sqrt{\left(-8+a^{2}+2 b+b^{2}+2 a(1+b)\right) \delta(-9+2 \delta)}\right)\right), \\
& \left.\frac{1}{9}(b-a) t(9(4-a-b)-(6-5 a-5 b) \delta-2 \sqrt{(9-2 \delta)(2-a-b)(4+a+b) \delta})\right\} \\
& \left\{-\frac{1}{9(-9+2 \delta)}(a-b) t\left(4(-3+a+b) \delta^{2}+9\left(-18+\sqrt{\left(-8+a^{2}+2 b+b^{2}+2 a(1+b)\right) \delta(-9+2 \delta)}\right)-\right.\right. \\
& \left.\delta\left(-90+18 a+18 b+7 \sqrt{\left(-8+a^{2}+2 b+b^{2}+2 a(1+b)\right) \delta(-9+2 \delta)}\right)\right), \\
& \left.\frac{1}{9}(-a+b) t(9(4-a-b)-(6-5 a-5 b) \delta-2 \sqrt{(2-a-b)(4+a+b)(9-2 \delta) \delta})\right\}
\end{aligned}
$$

Also, for the threshold value of $p_{B}(\mathrm{pb} 2)$, the point of $p_{A}$-element in Firm A's reaction function in (iii)' is

$$
\begin{aligned}
& \text { Simplify }\left[p_{A} \rightarrow p_{B}+\frac{(b-a) t((a+b)(3-2 \delta c)-2(3-\delta c))}{3} /\right. \\
& \left.p_{B} \rightarrow \frac{1}{9}(b-a) t(9(4-a-b)-(6-5 a-5 b) \delta-2 \sqrt{(9-2 \delta)(2-a-b)(4+a+b) \delta})\right] \\
& p_{A} \rightarrow \frac{1}{9}(a-b) t\left(-18+a \delta+b \delta+2 \sqrt{\left(-8+a^{2}+2 b+b^{2}+2 a(1+b)\right) \delta(-9+2 \delta)}\right)
\end{aligned}
$$

We can define the jumping point of Firm A's reaction function in (iii) as c3ja1

$$
\begin{gathered}
c 3 j a 2=\left\{\frac{1}{9}(a-b) t\left(-18+a \delta+b \delta+2 \sqrt{\left(-8+a^{2}+2 b+b^{2}+2 a(1+b)\right) \delta(-9+2 \delta)}\right),\right. \\
\left.\quad \frac{1}{9}(b-a) t(9(4-a-b)-(6-5 a-5 b) \delta-2 \sqrt{(9-2 \delta)(2-a-b)(4+a+b) \delta})\right\} \\
\left\{\frac{1}{9}(a-b) t\left(-18+a \delta+b \delta+2 \sqrt{\left(-8+a^{2}+2 b+b^{2}+2 a(1+b)\right) \delta(-9+2 \delta)}\right),\right. \\
\left.\frac{1}{9}(-a+b) t(9(4-a-b)-(6-5 a-5 b) \delta-2 \sqrt{(2-a-b)(4+a+b)(9-2 \delta) \delta})\right\}
\end{gathered}
$$

Next we show various examples of Firm A's true reaction function for different values of $a, b, \delta$, $\mathrm{t}$, and $\mathrm{k}$.

$$
\begin{aligned}
& a=0 \\
& b=1 \\
& \delta=1 / 2 \\
& t=1 \\
& k=2 \\
& 0 \\
& 1 \\
& \frac{1}{2} \\
& 1 \\
& 2
\end{aligned}
$$




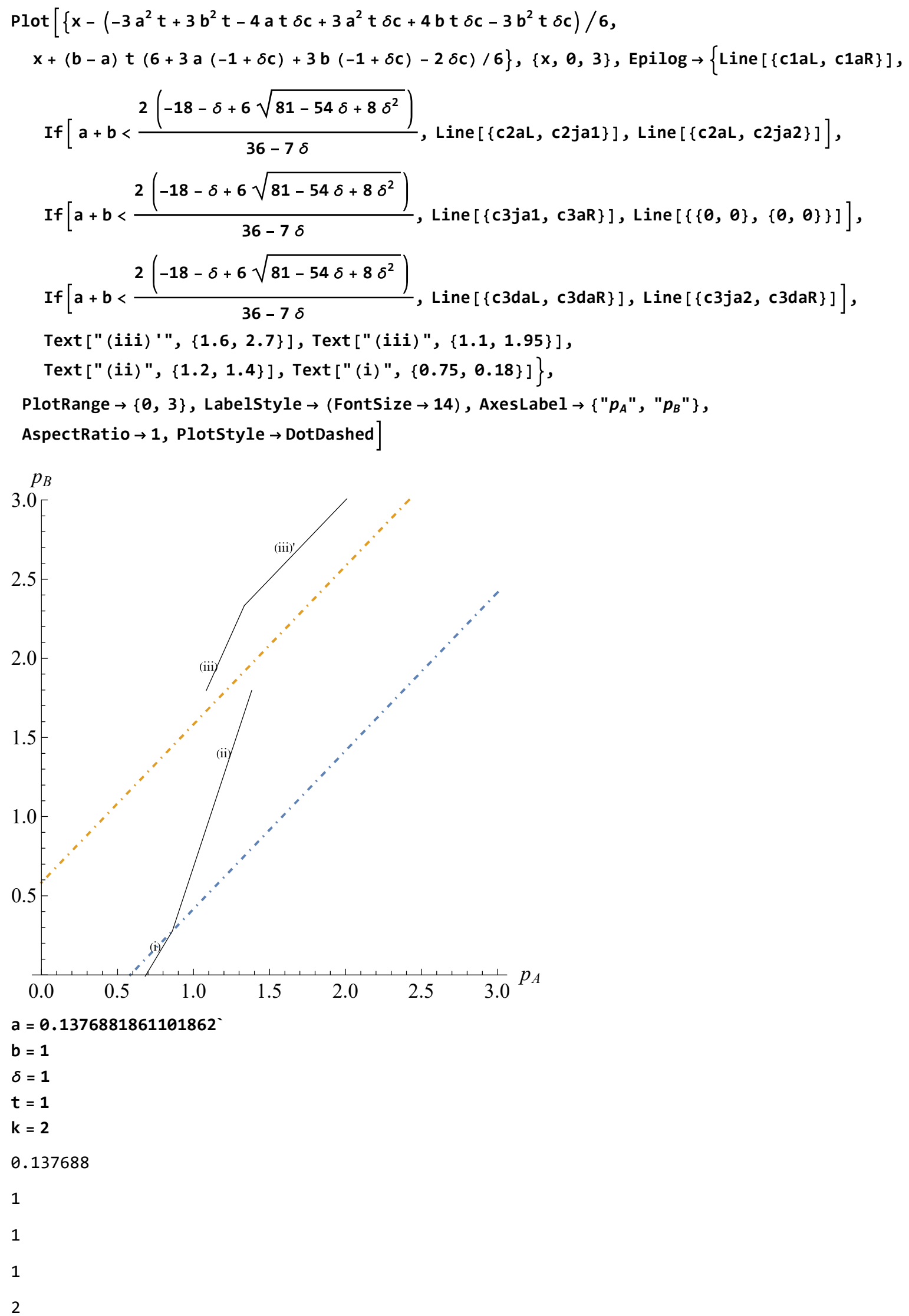




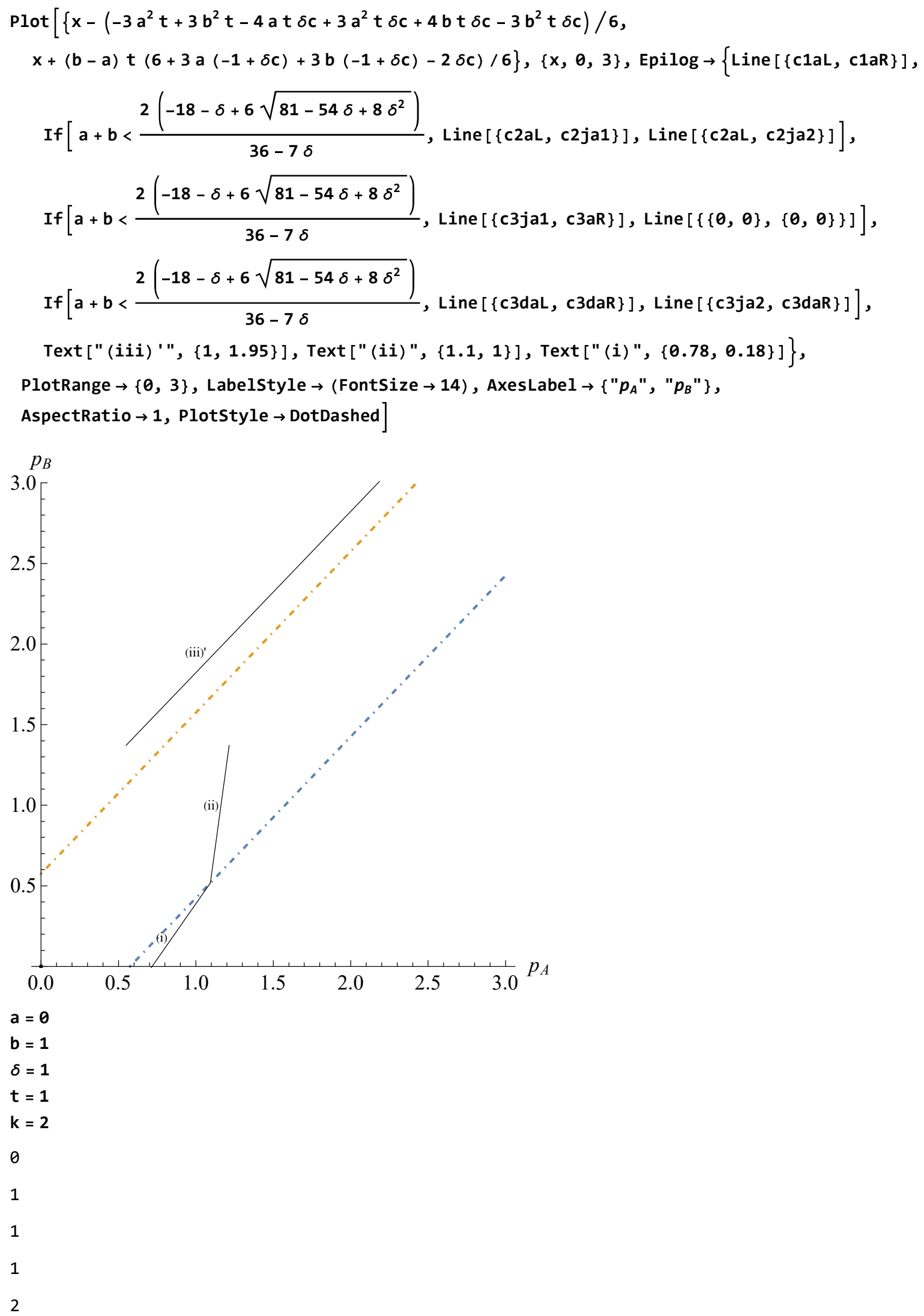




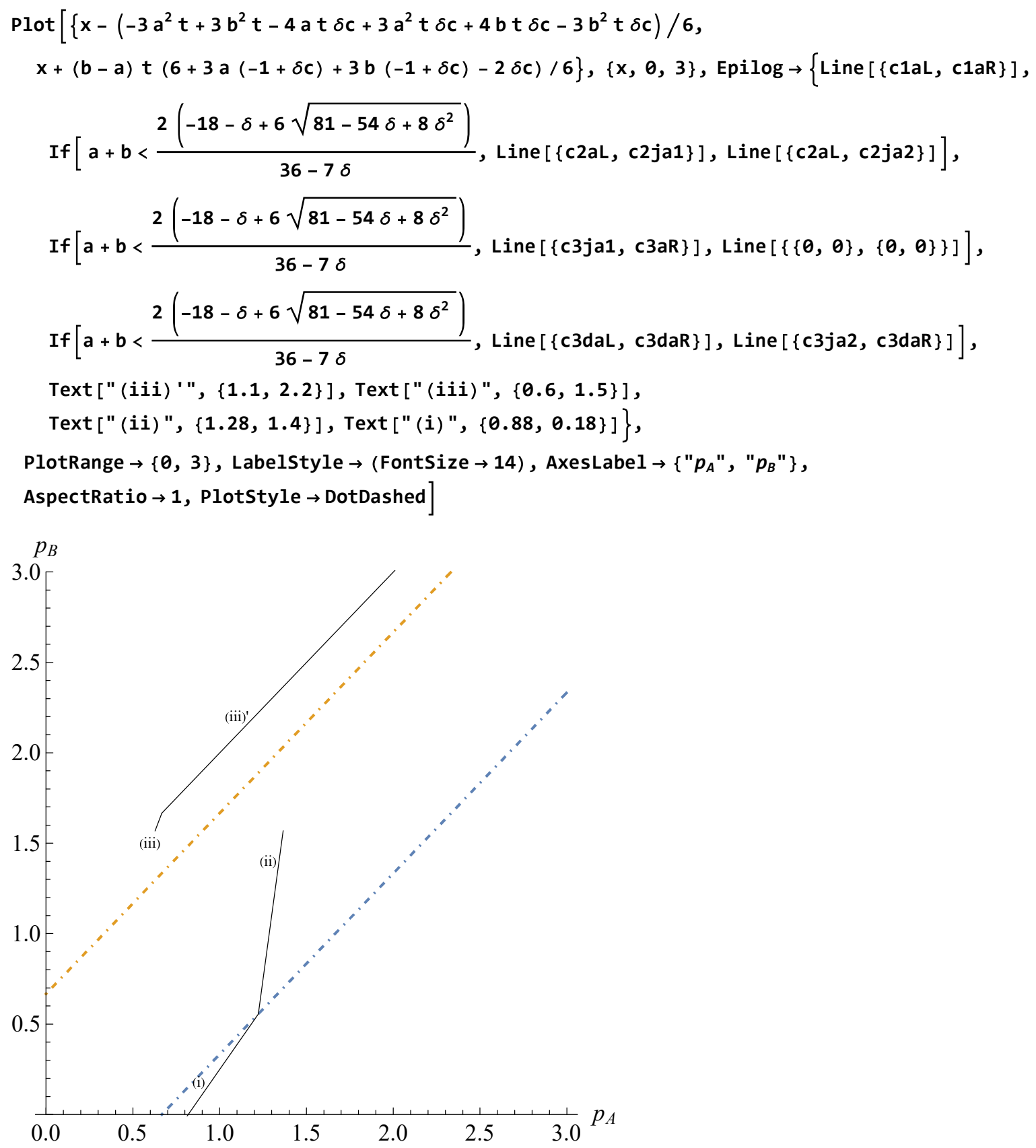

Clear [a, b, $\delta, t, k]$

We now turn to Firm B's reaction function in the three cases.

As before, we start with an example by setting $\mathrm{a}=0, \mathrm{~b}=1, \mathrm{t}=1$, and $\delta=1 / 2$. 


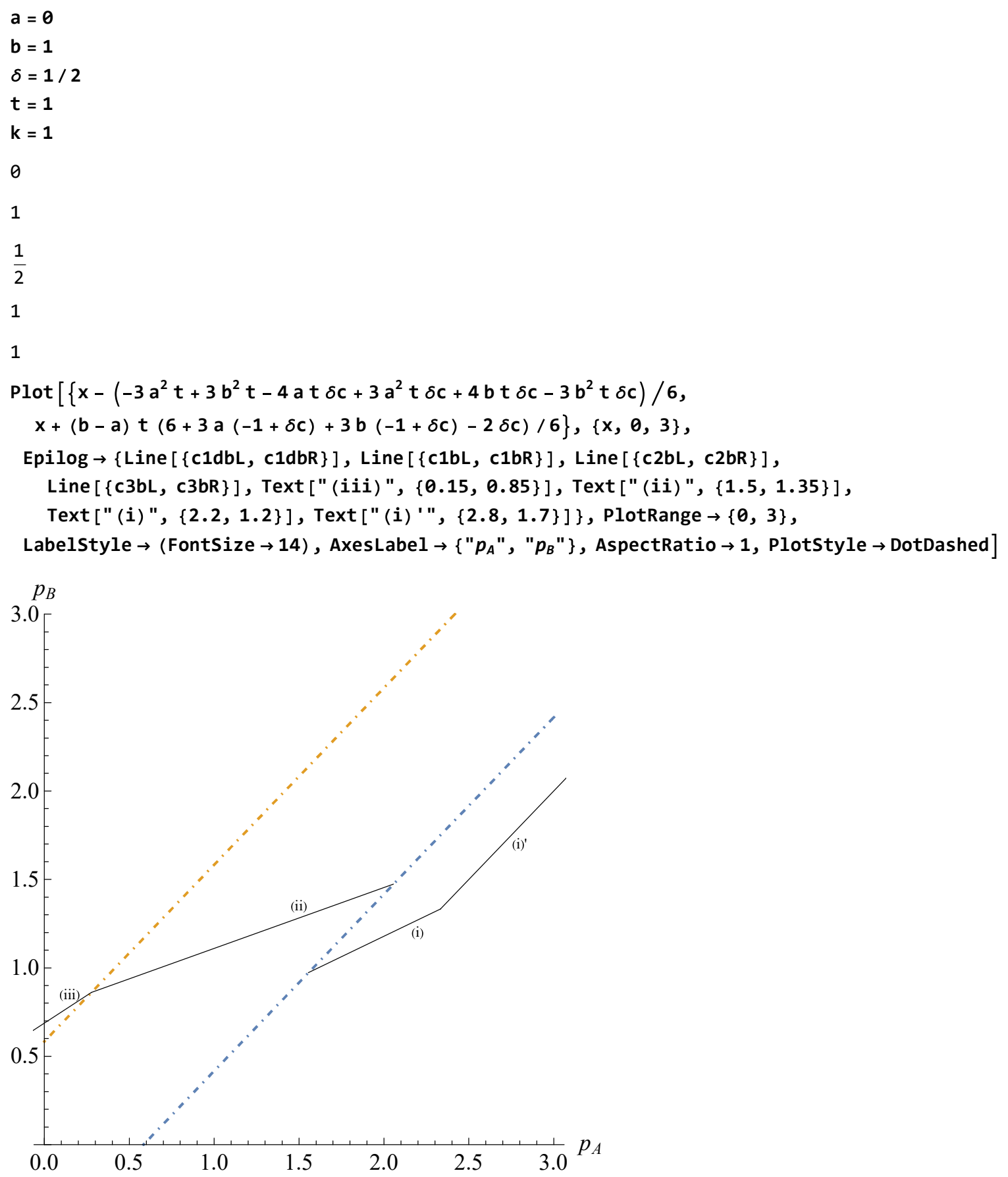

We find that for some $p_{A}$, there are two local optimal prices for Firm B. We can show that the multiplicity of local optimal prices always appears.

clear $[a, b, \delta, t, k]$

To show the multiplicity, we check the locations of the three endpoints: The right-hand endpoint in (ii) (c2bR) and the left-hand and right-hand endpoints in (i) (c1bL and c1bR) (see below) 


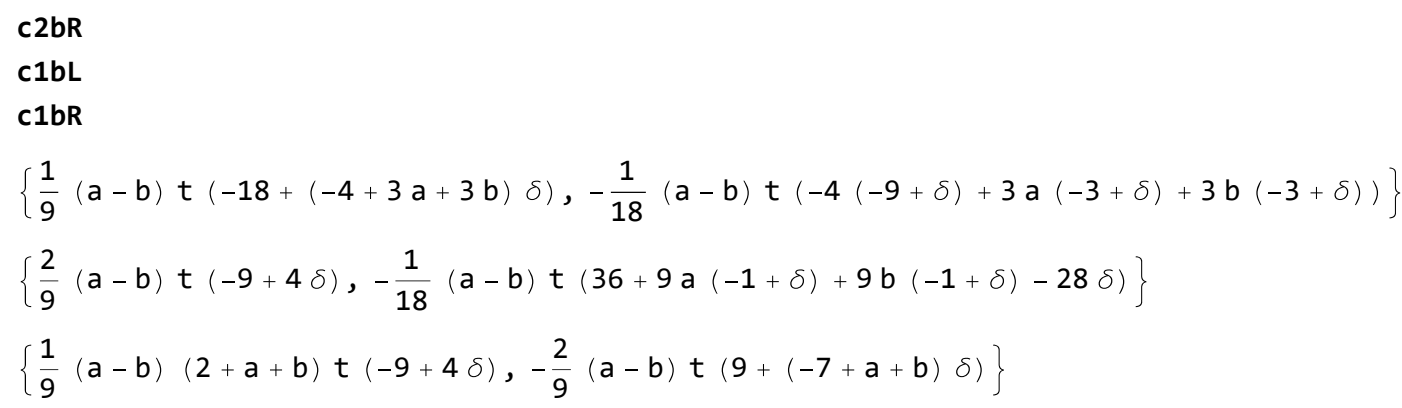

First, we compare the elements of the right-hand endpoint in (ii) and the left-hand endpoint in (i):

$$
\begin{aligned}
& \text { Factor }\left[\frac{1}{9}(a-b) t(-18+(-4+3 a+3 b) \delta)-\frac{2}{9}(a-b) t(-9+4 \delta)\right] \\
& \text { Factor }\left[-\frac{1}{18}(a-b) t(-4(-9+\delta)+3 a(-3+\delta)+3 b(-3+\delta))-\right. \\
& \left.\quad\left(-\frac{1}{18}(a-b) t(36+9 a(-1+\delta)+9 b(-1+\delta)-28 \delta)\right)\right] \\
& \frac{1}{3}(a-b)(-4+a+b) \text { t } \delta \\
& \frac{1}{3}(a-b)(-4+a+b) \text { t } \delta
\end{aligned}
$$

The outcome means that for any $\mathrm{a} \in[0,1], \mathrm{b} \in[0,1](\mathrm{a} \geq \mathrm{b}), \mathrm{t}$, and $\delta$, the right-hand endpoint in (ii) is located above the left-hand endpoint in (i) as in the above Figure.

Second, we compare the $p_{A}$-elements of the right-hand endpoint in (ii) and the right-hand endpoint in (i):

Factor $\left[\frac{1}{9}(a-b) t(-18+(-4+3 a+3 b) \delta)-\left(\frac{1}{9}(a-b)(2+a+b) t(-9+4 \delta)\right)\right]$

$-\frac{1}{9}(a-b) t(-9 a-9 b+12 \delta+a \delta+b \delta)$

The $p_{A}$-element of the right-hand endpoint in (ii) larger than that of the right-hand endpoint in (i) if and only if

$$
\frac{12 \delta}{9-\delta}>a+b
$$

If $\frac{12 \delta}{9-\delta} \leq \mathrm{a}+\mathrm{b}$, we simply compare the reaction function in (ii) and the reaction function in (i);

If $\frac{12 \delta}{9-\delta}>a+b$, in addition to the previous comparison, we also compare the reaction function in (ii) and the reaction function in (i)'. 


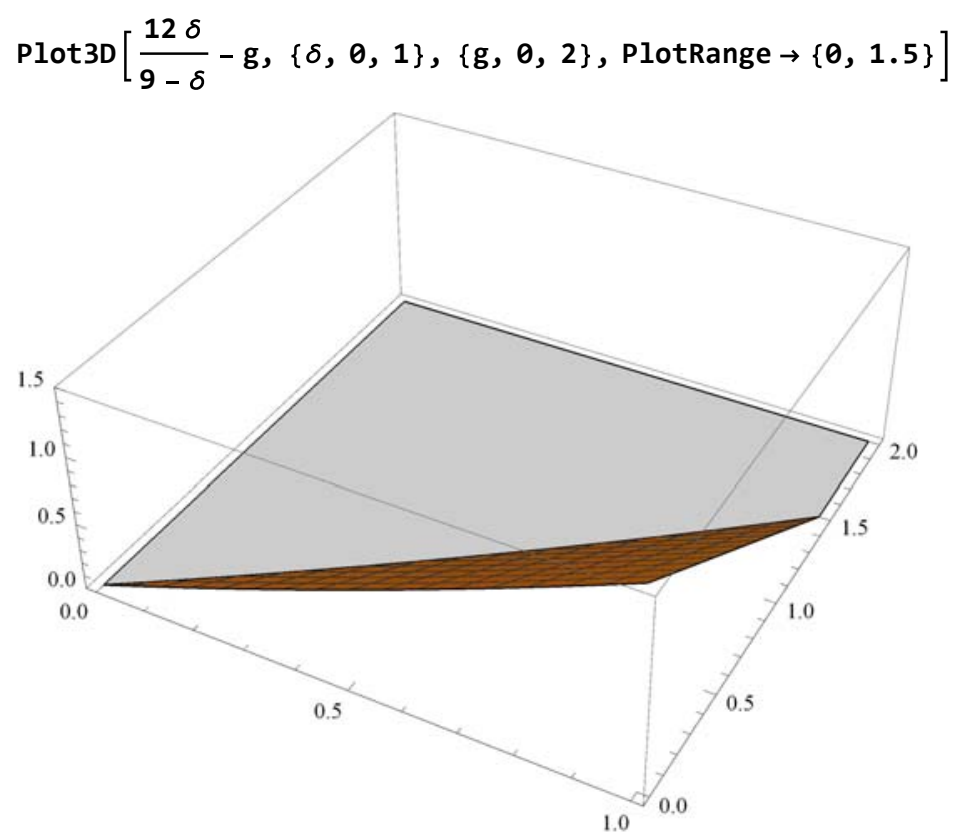

We need to find the global optimal price of Firm B, $p_{B}$, when there are two local optima for a given $p_{A}$. There is a price $p_{A}$ such that choosing the reaction function in (i) and choosing the reaction function in (ii) are indifferent for Firm B. This $p_{A}$ is the threshold in which choosing the reaction function in (i) is preferred by Firm B if $p_{A}$ is larger than the threshold $p_{A}$, otherwise, choosing the reaction function in (ii) is preferred by Firm B. We need to find the threshold value of $p_{A}$.

To check the threshold value of $p_{A}$ for Firm B's reaction function, we derive the profits under cases (i) and (ii).

The interior profit of firm $\mathrm{B}$ under case (ii) for $p_{A}$ is

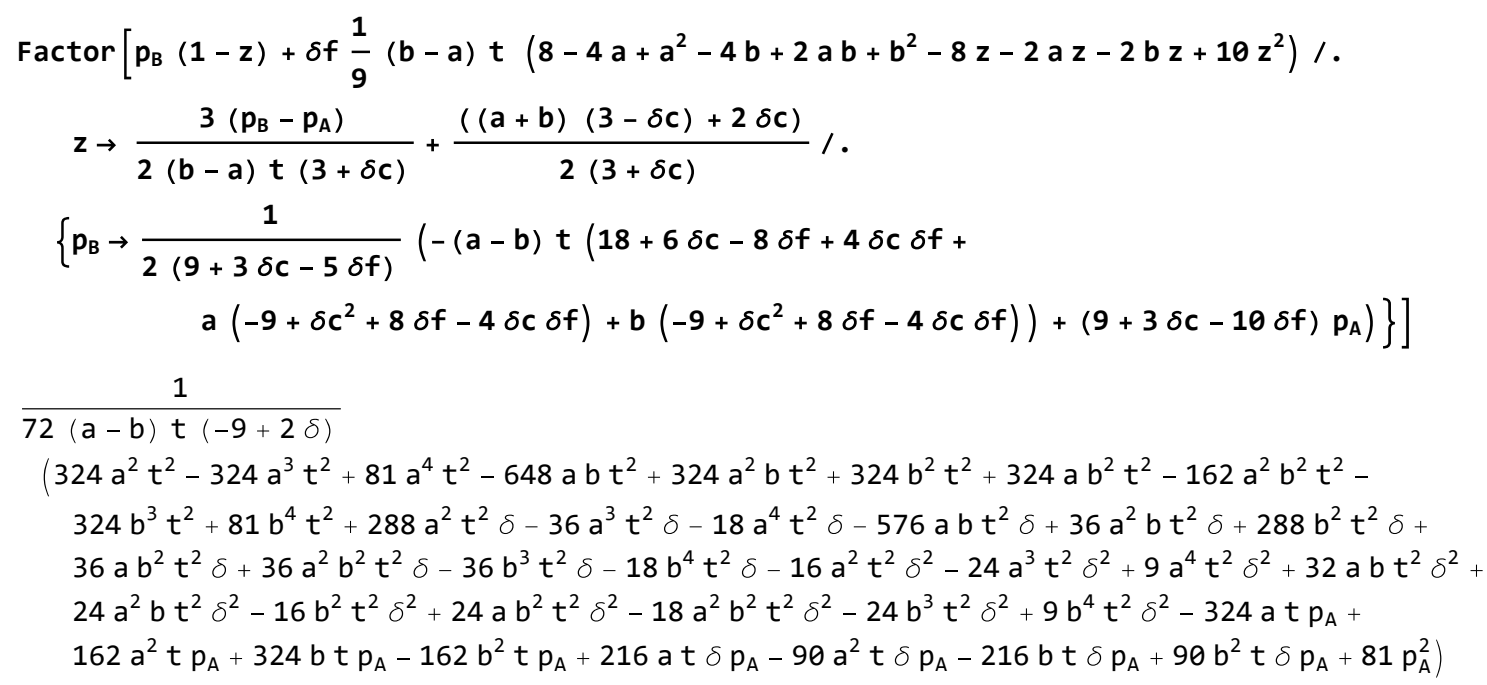

The interior profit of firm $\mathrm{B}$ under case (i) for $p_{B}$ 


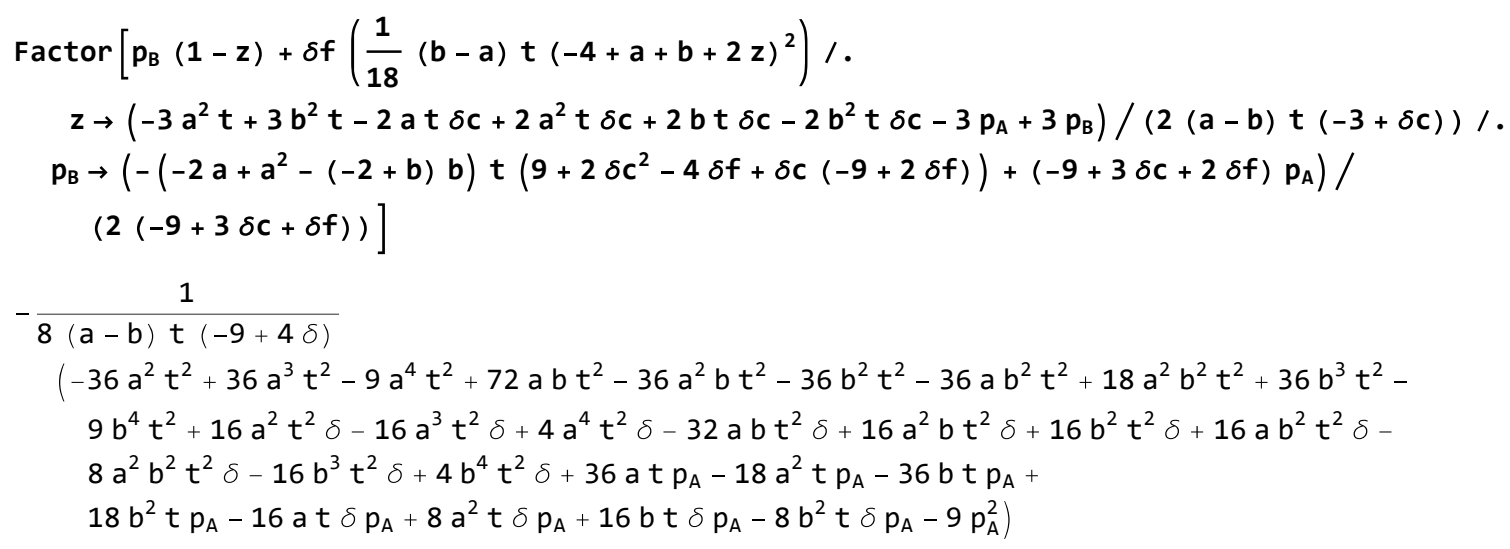

We derive the threshold value of $p_{B}$ by finding $p_{B}$ that equalizes the above two profits:

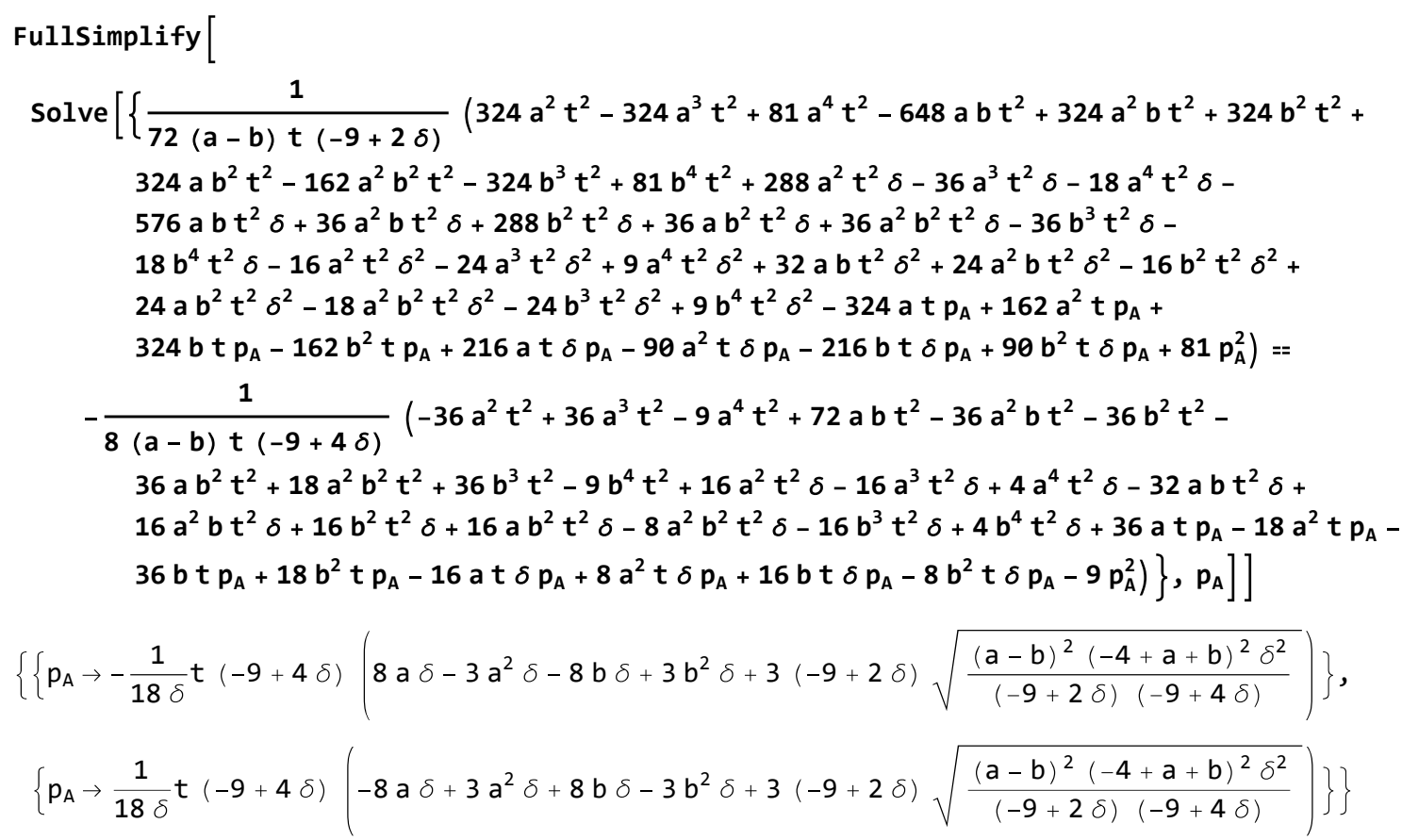

We can easily show that the former outcome is negative. So, we use the latter one.

We simplify the expression of the latter outcome, and obtain the following $p_{B}$ :

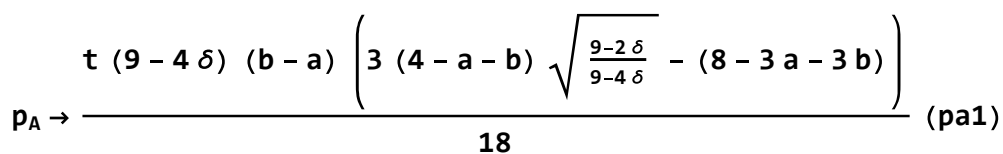

We rewrite the locations of the two endpoints: The right-hand endpoint in (ii) and the right-hand endpoint in (i) (see below)

$c 2 b R$

c1bR

$$
\begin{aligned}
& \left\{\frac{1}{9}(a-b) t(-18+(-4+3 a+3 b) \delta),-\frac{1}{18}(a-b) t(-4(-9+\delta)+3 a(-3+\delta)+3 b(-3+\delta))\right\} \\
& \left\{\frac{1}{9}(a-b)(2+a+b) t(-9+4 \delta),-\frac{2}{9}(a-b) t(9+(-7+a+b) \delta)\right\}
\end{aligned}
$$

We check the condition that the derived $p_{A}$ (pa1) is smaller than the $p_{A}$-element of the righthand endpoints in (i). 


$$
\begin{aligned}
& \text { Simplify }[ \\
& \text { Factor } \left.\left[\frac{1}{9}(a-b)(2+a+b) t(-9+4 \delta)-\frac{t(9-4 \delta)(b-a)\left(3(4-a-b) \sqrt{\frac{9-2 \delta}{9-4 \delta}}-(8-3 a-3 b)\right)}{18}\right]\right] \\
& \frac{1}{18}(a-b) t\left(12-b+a\left(-1+3 \sqrt{\frac{9-2 \delta}{9-4 \delta}}\right)-12 \sqrt{\frac{9-2 \delta}{9-4 \delta}}+3 b \sqrt{\frac{9-2 \delta}{9-4 \delta}}\right)(-9+4 \delta)
\end{aligned}
$$

This is positive if the following inequality holds

$(a+b)\left(-1+3 \sqrt{\frac{9-2 \delta}{9-4 \delta}}\right)>12\left(\sqrt{\frac{9-2 \delta}{9-4 \delta}}-1\right)$

$\operatorname{Plot}\left[12\left(\sqrt{\frac{9-2 \delta}{9-4 \delta}}-1\right) /\left(-1+3 \sqrt{\frac{9-2 \delta}{9-4 \delta}}\right),\{\delta, 0,1\}\right]$

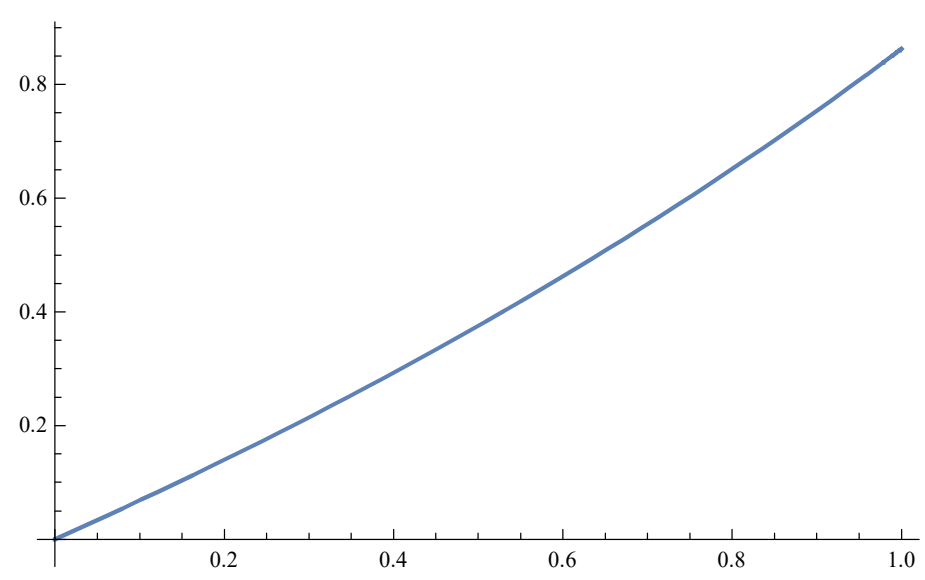

If $(a+b)\left(-1+3 \sqrt{\frac{9-2 \delta}{9-4 \delta}}\right)>12\left(\sqrt{\frac{9-2 \delta}{9-4 \delta}}-1\right)$ holds, the derived $p_{A}$ (pal) is on the interval between the left-hand and right-hand endpoints in (i). Otherwise, the reaction function in (ii) is always better than the interval between the left-hand and right-hand endpoints in (i).

Now we derive the profit of firm B under case (i)' for $p_{A}$

$$
\begin{aligned}
& \text { Factor }\left[p_{B}(1-z)+\delta f\left(\frac{1}{18}(b-a) t(-4+a+b+2 z)^{2}\right) /\right. \\
& \quad z \rightarrow\left(-3 a^{2} t+3 b^{2} t-2 a t \delta c+2 a^{2} t \delta c+2 b t \delta c-2 b^{2} t \delta c-3 p_{A}+3 p_{B}\right) /(2(a-b) t(-3+\delta c)) / \\
& \left.p_{B} \rightarrow p_{A}-\frac{(b-a) t((a+b)(3-2 \delta c)+2 \delta c)}{3}\right] \\
& \frac{1}{18}\left(18 a^{2} t-18 b^{2} t-4 a t \delta-4 a^{2} t \delta-a^{3} t \delta+4 b t \delta-a^{2} b t \delta+4 b^{2} t \delta+a b^{2} t \delta+b^{3} t \delta+18 p_{A}\right)
\end{aligned}
$$

We derive the threshold value of $p_{A}$ by finding $p_{A}$ that equalizes the two profits in cases (ii) and (i)': 


$$
\begin{aligned}
& \text { Fullsimplify [ } \\
& \text { Solve }\left[\left\{\frac { 1 } { 7 2 ( a - b ) t ( - 9 + 2 \delta ) } \left(324 a^{2} t^{2}-324 a^{3} t^{2}+81 a^{4} t^{2}-648 a b t^{2}+324 a^{2} b t^{2}+324 b^{2} t^{2}+\right.\right.\right. \\
& 324 a b^{2} t^{2}-162 a^{2} b^{2} t^{2}-324 b^{3} t^{2}+81 b^{4} t^{2}+288 a^{2} t^{2} \delta-36 a^{3} t^{2} \delta-18 a^{4} t^{2} \delta- \\
& 576 a b t^{2} \delta+36 a^{2} b t^{2} \delta+288 b^{2} t^{2} \delta+36 a b^{2} t^{2} \delta+36 a^{2} b^{2} t^{2} \delta-36 b^{3} t^{2} \delta-18 b^{4} t^{2} \delta- \\
& 16 a^{2} t^{2} \delta^{2}-24 a^{3} t^{2} \delta^{2}+9 a^{4} t^{2} \delta^{2}+32 a b t^{2} \delta^{2}+24 a^{2} b t^{2} \delta^{2}-16 b^{2} t^{2} \delta^{2}+24 a b^{2} t^{2} \delta^{2}- \\
& 18 a^{2} b^{2} t^{2} \delta^{2}-24 b^{3} t^{2} \delta^{2}+9 b^{4} t^{2} \delta^{2}-324 a t p_{A}+162 a^{2} t p_{A}+324 b t p_{A}-162 b^{2} t p_{A}+ \\
& \left.216 a \text { t } \delta p_{A}-90 a^{2} t \delta p_{A}-216 b t \delta p_{A}+90 b^{2} t \delta p_{A}+81 p_{A}^{2}\right)=\frac{1}{18}\left(18 a^{2} t-18 b^{2} t-\right. \\
& \left.\left.\left.\left.4 a t \delta-4 a^{2} t \delta-a^{3} t \delta+4 b t \delta-a^{2} b t \delta+4 b^{2} t \delta+a b^{2} t \delta+b^{3} t \delta+18 p_{A}\right)\right\}, p_{A}\right]\right] \\
& \left\{\left\{p_{A} \rightarrow \frac{1}{9}(a-b) t\right.\right. \\
& \left.\left(-9 a-9(2+b)-4 \delta+5(a+b) \delta+18 \sqrt{\frac{(-6+a+b)(a+b) \delta}{-9+2 \delta}}-4 \delta \sqrt{\frac{(-6+a+b)(a+b) \delta}{-9+2 \delta}}\right)\right\}, \\
& \left\{p _ { A } \rightarrow \frac { 1 } { 9 } ( a - b ) t \left(-9(2+a+b)+(-4+5 a+5 b) \delta-18 \sqrt{\frac{(-6+a+b)(a+b) \delta}{-9+2 \delta}}+\right.\right. \\
& \left.\left.\left.4 \delta \sqrt{\frac{(-6+a+b)(a+b) \delta}{-9+2 \delta}}\right)\right\}\right\}
\end{aligned}
$$

We pick up the first outcome as the threshold $p_{A}$.

$$
\begin{aligned}
& \mathrm{p}_{A} \rightarrow \frac{1}{9}(a-b) t \\
& \left(-9 a-9(2+b)-4 \delta+5(a+b) \delta+18 \sqrt{\frac{(-6+a+b)(a+b) \delta}{-9+2 \delta}}-4 \delta \sqrt{\frac{(-6+a+b)(a+b) \delta}{-9+2 \delta}}\right)
\end{aligned}
$$

We check the condition that the derived $p_{A}(\mathrm{pa} 2)$ is above the $p_{A}$-element of the right-hand endpoints in (i).

$$
\begin{aligned}
& \text { Simplify }\left[\text { Factor } \left[\frac{1}{9}(a-b) t\right.\right. \\
& \quad\left(-9 a-9(2+b)-4 \delta+5(a+b) \delta+18 \sqrt{\frac{(-6+a+b)(a+b) \delta}{-9+2 \delta}}-4 \delta \sqrt{\frac{(-6+a+b)(a+b) \delta}{-9+2 \delta}}\right)- \\
& \left.\left.\quad \frac{1}{9}(a-b)(2+a+b) t(-9+4 \delta)\right]\right] \\
& \frac{1}{9}(a-b) t\left(-12 \delta+a \delta+b \delta+18 \sqrt{\frac{(-6+a+b)(a+b) \delta}{-9+2 \delta}}-4 \delta \sqrt{\frac{(-6+a+b)(a+b) \delta}{-9+2 \delta}}\right)
\end{aligned}
$$

By setting $\mathrm{a}+\mathrm{b}$ by $\mathrm{g}$, we check the value between the large parentheses: 


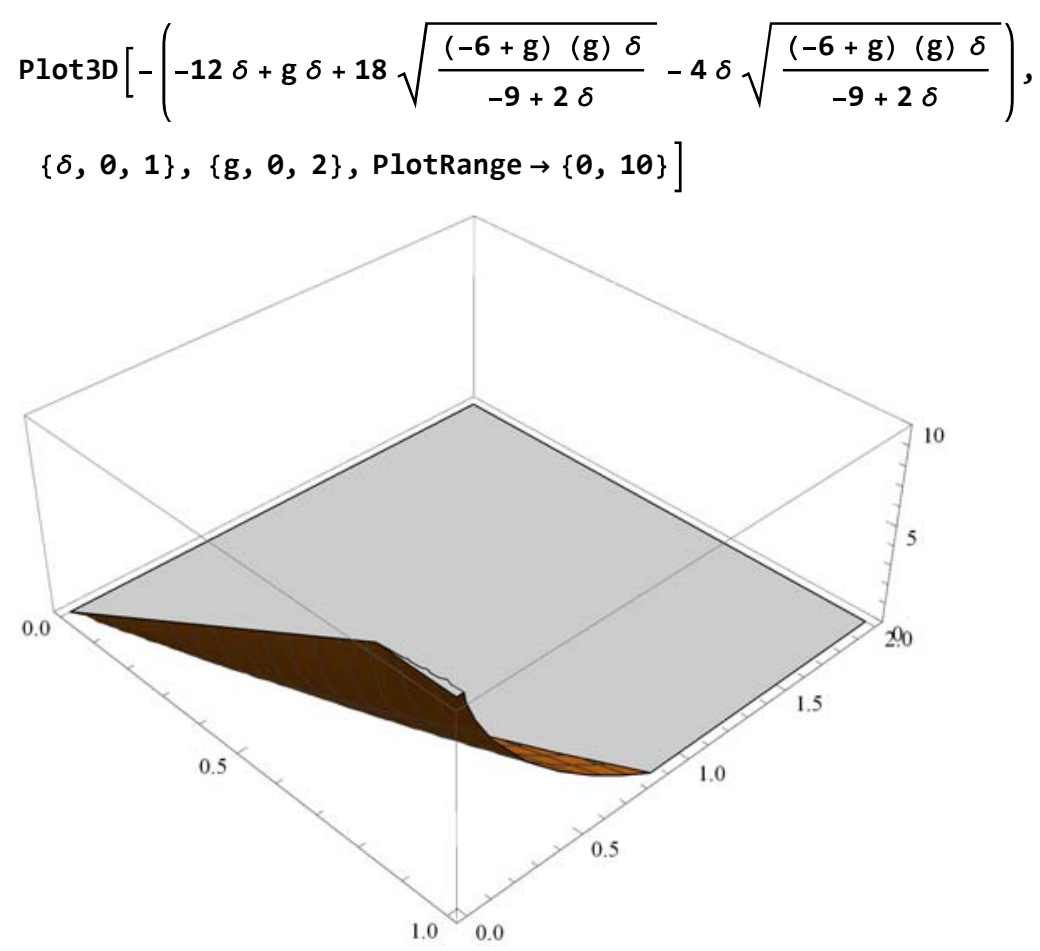

We check the condition that the value between the large parentheses is negative:

$$
\begin{aligned}
& \text { Solve }\left[\left(-12 \delta+g \delta+18 \sqrt{\frac{(-6+g)(g) \delta}{-9+2 \delta}}-4 \delta \sqrt{\frac{(-6+g)(g) \delta}{-9+2 \delta}}\right)=0, g\right] \\
& \left\{\left\{g \rightarrow \frac{12\left(-9+\delta-\sqrt{81-54 \delta+8 \delta^{2}}\right)}{-36+7 \delta}\right\},\left\{g \rightarrow \frac{12\left(-9+\delta+\sqrt{81-54 \delta+8 \delta^{2}}\right)}{-36+7 \delta}\right\}\right\} \\
& \text { If } g=a+b<\frac{12\left(-9+\delta+\sqrt{81-54 \delta+8 \delta^{2}}\right)}{-36+7 \delta},
\end{aligned}
$$

the derived $p_{A}$ (pa2) is above the $p_{A}$-element of the right-hand endpoints in (i).

This coincides with the following condition that the derived $p_{A}$ (pa1) is above the $p_{A}$-element of the right-hand endpoints in (i).

$\operatorname{Plot}\left[\frac{12\left(-9+\delta+\sqrt{81-54 \delta+8 \delta^{2}}\right)}{-36+7 \delta},\{\delta, 0,1\}\right]$

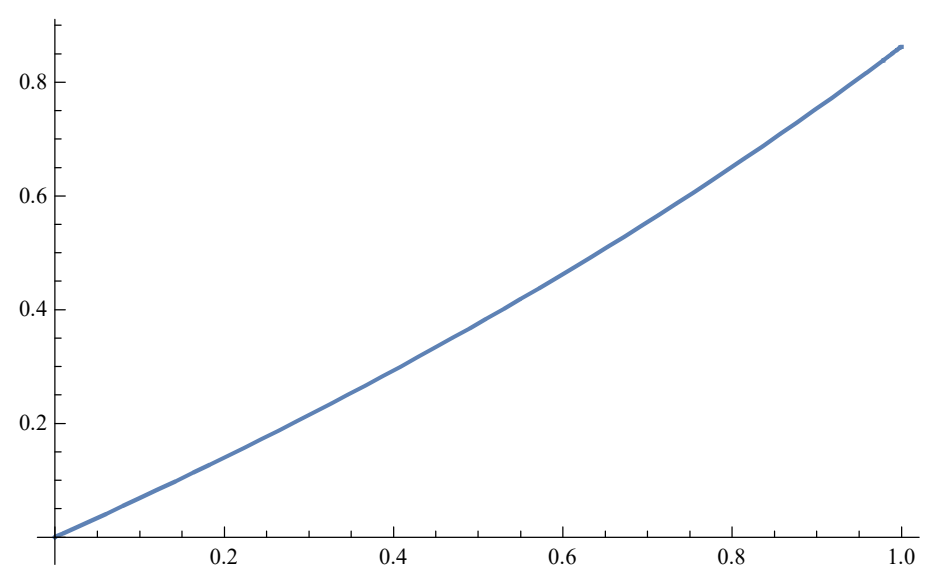

If $(\mathrm{a}+\mathrm{b})>\frac{12\left(-9+\delta+\sqrt{81-54 \delta+8 \delta^{2}}\right)}{-36+7 \delta}$ holds, the threshold $p_{B}$ is on the line segment between the left- 
hand and right-hand endpoints in (i). The threshold is given as

$p_{A} \rightarrow \frac{t(9-4 \delta)(b-a)\left(3(4-a-b) \sqrt{\frac{9-2 \delta}{9-4 \delta}}-(8-3 a-3 b)\right)}{18}$

The reaction function of Firm B in (i) is

$p_{B} \rightarrow \frac{1}{2(9-3 \delta c-\delta f)}\left(((b-a)(2-a-b)) t\left(9+2 \delta c^{2}-4 \delta f+\delta c(-9+2 \delta f)\right)+(9-3 \delta c-2 \delta f) p_{A}\right)$

We substitute $p_{A}$ into $p_{B}$ :

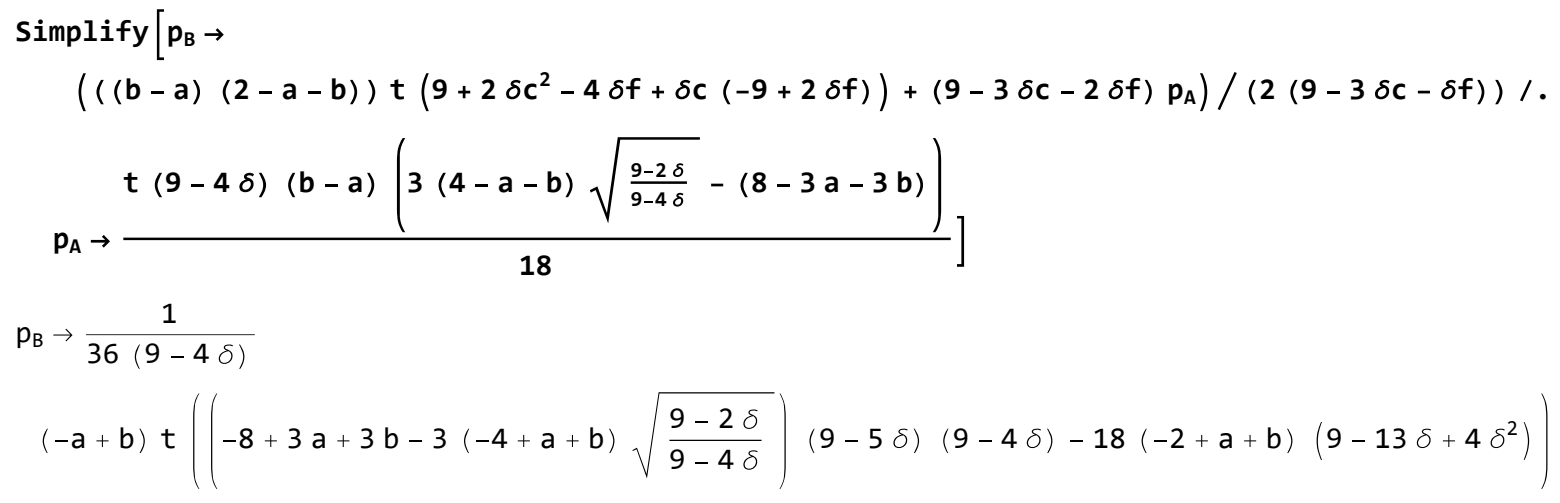

We can define the jumping point of Firm B's reaction function in (i) as c1jb1

$$
\begin{aligned}
& c 1 j b 1=\left\{\frac{t(9-4 \delta)(b-a)\left(3(4-a-b) \sqrt{\frac{9-2 \delta}{9-4 \delta}}-(8-3 a-3 b)\right.}{18}, \frac{1}{36(9-4 \delta)}(-a+b) t\right. \\
& \left.\quad\left(\left(-8+3 a+3 b-3(-4+a+b) \sqrt{\frac{9-2 \delta}{9-4 \delta}}\right)(9-5 \delta)(9-4 \delta)-18(-2+a+b)\left(9-13 \delta+4 \delta^{2}\right)\right)\right\} \\
& \left\{\frac{1}{18}(-a+b) t\left(-8+3 a+3 b+3(4-a-b) \sqrt{\frac{9-2 \delta}{9-4 \delta}}\right)(9-4 \delta), \frac{1}{36(9-4 \delta)}(-a+b) t\right. \\
& \left.\left(\left(-8+3 a+3 b-3(-4+a+b) \sqrt{\frac{9-2 \delta}{9-4 \delta}}\right)(9-5 \delta)(9-4 \delta)-18(-2+a+b)\left(9-13 \delta+4 \delta^{2}\right)\right)\right\}
\end{aligned}
$$

The reaction function of Firm B in (ii) is

$$
\begin{aligned}
p_{B} \rightarrow & \frac{1}{2(9+3 \delta c-5 \delta f)} \\
& \left(-(a-b) t\left(18+6 \delta c-8 \delta f+4 \delta c \delta f+a\left(-9+\delta c^{2}+8 \delta f-4 \delta c \delta f\right)+b\left(-9+\delta c^{2}+8 \delta f-4 \delta c \delta f\right)\right)+\right. \\
& \left.(9+3 \delta c-10 \delta f) p_{A}\right)
\end{aligned}
$$

We substitute $p_{A}$ into $p_{B}$ :

$$
\begin{aligned}
& \text { Simplify }\left[p_{B} \rightarrow \frac{1}{2(9+3 \delta c-5 \delta f)}\right. \\
& \begin{array}{l}
\left(-(a-b) t\left(18+6 \delta c-8 \delta f+4 \delta c \delta f+a\left(-9+\delta c^{2}+8 \delta f-4 \delta c \delta f\right)+b\left(-9+\delta c^{2}+8 \delta f-4 \delta c \delta f\right)\right)+\right. \\
\left.\left.(9+3 \delta c-10 \delta f) p_{A}\right) / \cdot p_{A} \rightarrow \frac{t(9-4 \delta)(b-a)\left(3(4-a-b) \sqrt{\frac{9-2 \delta}{9-4 \delta}}-(8-3 a-3 b)\right)}{18}\right] \\
p_{B} \rightarrow \frac{1}{126}(-a+b) t\left(-36(-5+a+b)+\left(-8+3 a+3 b-3(-4+a+b) \sqrt{\frac{9-2 \delta}{9-4 \delta}}\right)(9-4 \delta)\right)
\end{array}
\end{aligned}
$$


We can define the jumping point of Firm B's reaction function in (ii) as c2jb1

$$
\begin{aligned}
& c 2 j b 1=\left\{\frac{t(9-4 \delta)(b-a)\left(3(4-a-b) \sqrt{\frac{9-2 \delta}{9-4 \delta}}-(8-3 a-3 b)\right)}{18},\right. \\
& \left.\frac{1}{126}(-a+b) t\left(-36(-5+a+b)+\left(-8+3 a+3 b-3(-4+a+b) \sqrt{\frac{9-2 \delta}{9-4 \delta}}\right)(9-4 \delta)\right)\right\} \\
& \left\{\frac{1}{18}(-a+b) t\left(-8+3 a+3 b+3(4-a-b) \sqrt{\frac{9-2 \delta}{9-4 \delta}}\right)(9-4 \delta),\right. \\
& \left.\frac{1}{126}(-a+b) t\left(-36(-5+a+b)+\left(-8+3 a+3 b-3(-4+a+b) \sqrt{\frac{9-2 \delta}{9-4 \delta}}\right)(9-4 \delta)\right)\right\}
\end{aligned}
$$

If $(\mathrm{a}+\mathrm{b}) \leq \frac{12\left(-9+\delta+\sqrt{81-54 \delta+8 \delta^{2}}\right)}{-36+7 \delta}$ holds, the threshold $p_{A}$ is on the half-line starting from the right-hand endpoints in (i), that is, case (i)'. The threshold is given as

$$
\begin{aligned}
& p_{A} \rightarrow \frac{1}{9}(a-b) t \\
& \quad\left(-9 a-9(2+b)-4 \delta+5(a+b) \delta+18 \sqrt{\frac{(-6+a+b)(a+b) \delta}{-9+2 \delta}}-4 \delta \sqrt{\frac{(-6+a+b)(a+b) \delta}{-9+2 \delta}}\right)
\end{aligned}
$$

The reaction functions of Firm B in (i)' and (ii) are

$$
\begin{aligned}
& p_{B} \rightarrow p_{A}-\frac{(b-a) t((a+b)(3-2 \delta c)+2 \delta c)}{3} \\
& p_{B} \rightarrow\left((b-a) t\left(2(9+3 \delta c-4 \delta f+2 \delta c \delta f)+(a+b)\left(-9+\delta c^{2}+8 \delta f-4 \delta c \delta f\right)\right)+(9+3 \delta c-10 \delta f) p_{A}\right) / \\
&(2(9+3 \delta c-5 \delta f))
\end{aligned}
$$

We substitute $p_{A}$ into $p_{B}$ :

$$
\begin{aligned}
& \text { Simplify }\left[p_{B} \rightarrow p_{A}-\frac{(b-a) t((a+b)(3-2 \delta c)+2 \delta c)}{3} / \cdot p_{A} \rightarrow \frac{1}{9}(a-b) t\right. \\
& \left.\left(-9 a-9(2+b)-4 \delta+5(a+b) \delta+18 \sqrt{\frac{(-6+a+b)(a+b) \delta}{-9+2 \delta}}-4 \delta \sqrt{\frac{(-6+a+b)(a+b) \delta}{-9+2 \delta}}\right)\right] \\
& \text { Simplify }\left[p _ { B } \rightarrow \left((b-a) t\left(2(9+3 \delta c-4 \delta f+2 \delta c \delta f)+(a+b)\left(-9+\delta c^{2}+8 \delta f-4 \delta c \delta f\right)\right)+\right.\right. \\
& \left.(9+3 \delta c-10 \delta f) p_{A}\right) /(2(9+3 \delta c-5 \delta f)) / . p_{A} \rightarrow \frac{1}{9}(a-b) t \\
& \left.\left(-9 a-9(2+b)-4 \delta+5(a+b) \delta+18 \sqrt{\frac{(-6+a+b)(a+b) \delta}{-9+2 \delta}}-4 \delta \sqrt{\frac{(-6+a+b)(a+b) \delta}{-9+2 \delta}}\right)\right] \\
& \mathrm{p}_{\mathrm{B}} \rightarrow-\frac{1}{9}(\mathrm{a}-\mathrm{b}) \mathrm{t}\left(18-2 \delta+\mathrm{a} \delta+\mathrm{b} \delta-18 \sqrt{\frac{(-6+\mathrm{a}+\mathrm{b})(\mathrm{a}+\mathrm{b}) \delta}{-9+2 \delta}}+4 \delta \sqrt{\frac{(-6+\mathrm{a}+\mathrm{b})(\mathrm{a}+\mathrm{b}) \delta}{-9+2 \delta}}\right) \\
& p_{B} \rightarrow \frac{1}{9}(a-b) t\left(-18+2 \delta+2 a \delta+2 b \delta+9 \sqrt{\frac{(-6+a+b)(a+b) \delta}{-9+2 \delta}}-7 \delta \sqrt{\frac{(-6+a+b)(a+b) \delta}{-9+2 \delta}}\right)
\end{aligned}
$$

We can define the jumping point of Firm B's reaction function in (i) as c1jb2 


$$
\begin{aligned}
& c 1 j b 2=\left\{\frac{1}{9}(a-b) t\right. \\
& \left.\quad-9 a-9(2+b)-4 \delta+5(a+b) \delta+18 \sqrt{\frac{(-6+a+b)(a+b) \delta}{-9+2 \delta}}-4 \delta \sqrt{\frac{(-6+a+b)(a+b) \delta}{-9+2 \delta}}\right), \\
& \left.\quad-\frac{1}{9}(a-b) t\left(18-2 \delta+a \delta+b \delta-18 \sqrt{\frac{(-6+a+b)(a+b) \delta}{-9+2 \delta}}+4 \delta \sqrt{\frac{(-6+a+b)(a+b) \delta}{-9+2 \delta}}\right)\right\} \\
& \left\{\frac{1}{9}(a-b) t\right. \\
& \left(-9 a-9(2+b)-4 \delta+5(a+b) \delta+18 \sqrt{\frac{(-6+a+b)(a+b) \delta}{-9+2 \delta}}-4 \delta \sqrt{\frac{(-6+a+b)(a+b) \delta}{-9+2 \delta}}\right), \\
& \left.-\frac{1}{9}(a-b) t\left(18-2 \delta+a \delta+b \delta-18 \sqrt{\frac{(-6+a+b)(a+b) \delta}{-9+2 \delta}}+4 \delta \sqrt{\frac{(-6+a+b)(a+b) \delta}{-9+2 \delta}}\right)\right\}
\end{aligned}
$$

Also, we can define the jumping point of Firm B's reaction function in (ii) as c2jb2

$$
\begin{aligned}
& c 2 j b 2=\left\{\frac{1}{9}(a-b) t\right. \\
& \left(-9 a-9(2+b)-4 \delta+5(a+b) \delta+18 \sqrt{\frac{(-6+a+b)(a+b) \delta}{-9+2 \delta}}-4 \delta \sqrt{\frac{(-6+a+b)(a+b) \delta}{-9+2 \delta}}\right), \\
& \left.\quad \frac{1}{9}(a-b) t\left(-18+2 \delta+2 a \delta+2 b \delta+9 \sqrt{\frac{(-6+a+b)(a+b) \delta}{-9+2 \delta}}-7 \delta \sqrt{\frac{(-6+a+b)(a+b) \delta}{-9+2 \delta}}\right)\right\} \\
& \left\{\frac{1}{9}(a-b) t\right. \\
& \left(-9 a-9(2+b)-4 \delta+5(a+b) \delta+18 \sqrt{\frac{(-6+a+b)(a+b) \delta}{-9+2 \delta}}-4 \delta \sqrt{\frac{(-6+a+b)(a+b) \delta}{-9+2 \delta}}\right), \\
& \left.\frac{1}{9}(a-b) t\left(-18+2 \delta+2 a \delta+2 b \delta+9 \sqrt{\frac{(-6+a+b)(a+b) \delta}{-9+2 \delta}}-7 \delta \sqrt{\frac{(-6+a+b)(a+b) \delta}{-9+2 \delta}}\right)\right\}
\end{aligned}
$$

As before, we now show various examples of Firm B's true reaction function for different values of $\mathrm{a}, \mathrm{b}, \delta$, $\mathrm{t}$, and $\mathrm{k}$.

$$
\begin{aligned}
& a=0 \\
& b=1 \\
& \delta=1 / 2 \\
& t=1 \\
& k=2 \\
& 0 \\
& 1 \\
& \frac{1}{2} \\
& 1 \\
& 2
\end{aligned}
$$




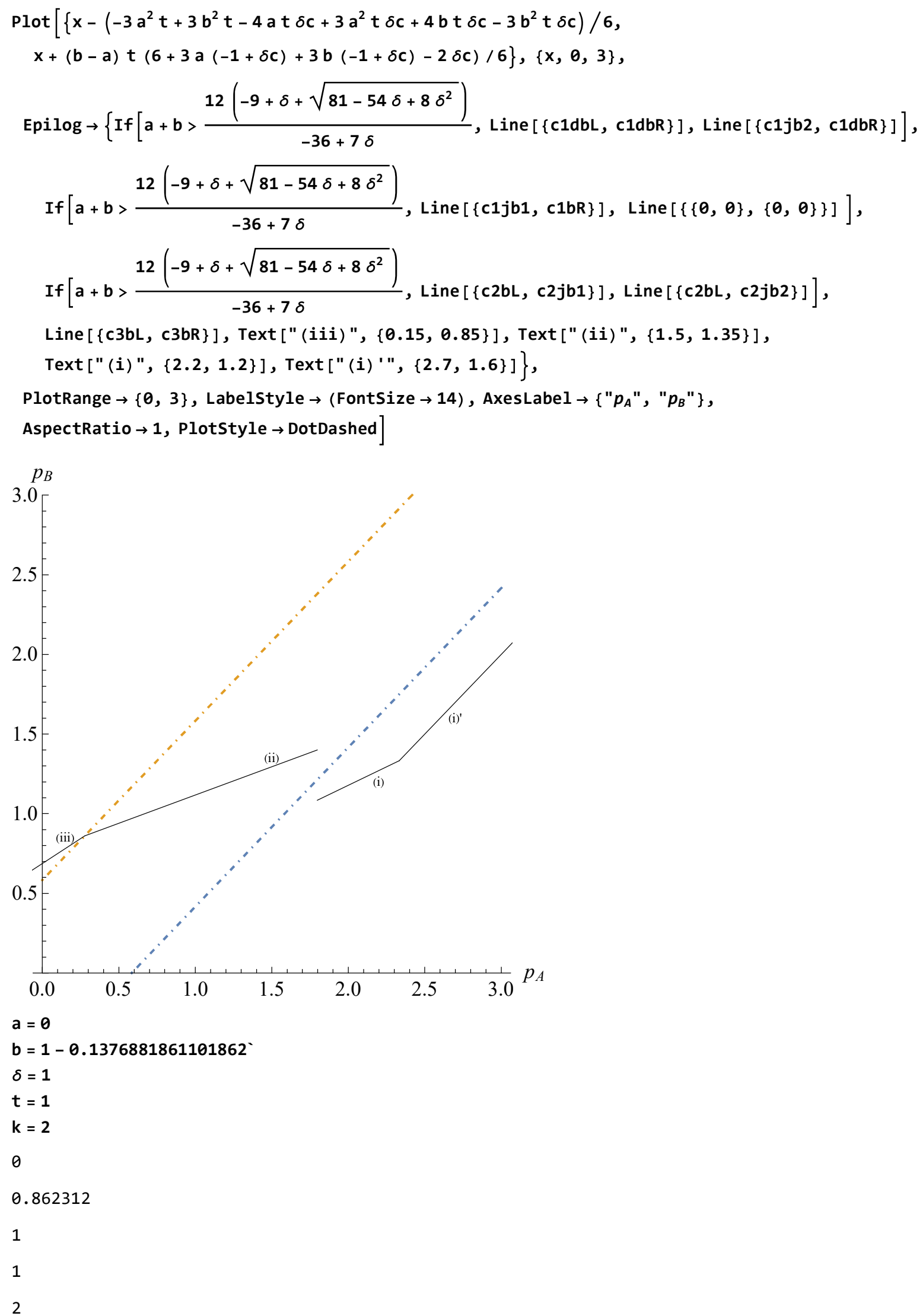




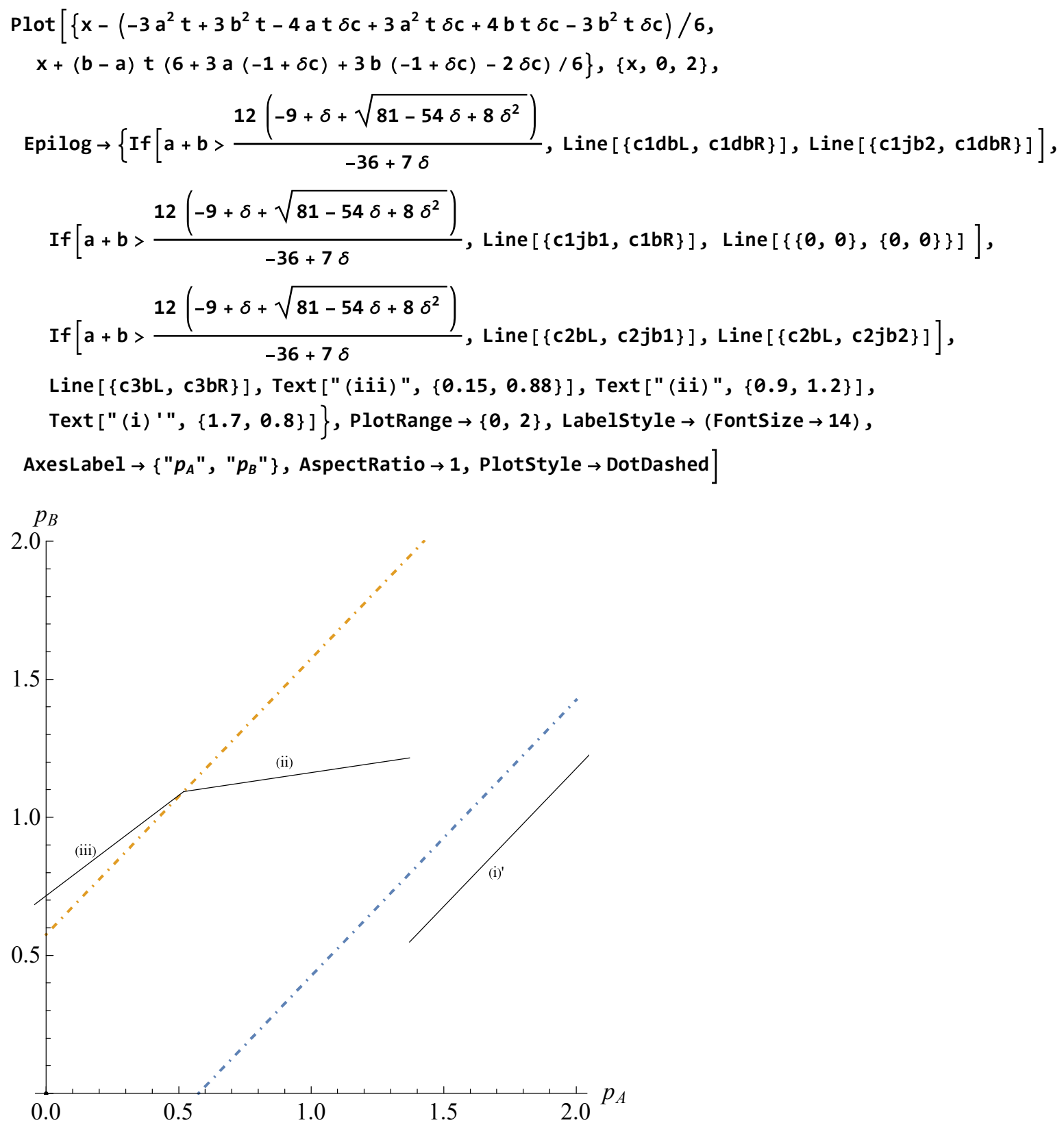

We now show an example in which there is no intersection between the two firms' reaction functions.

$a=0$
$b=0.001$
$\delta=1$
$t=1$
$k=2$
0
0.001
1
1
2



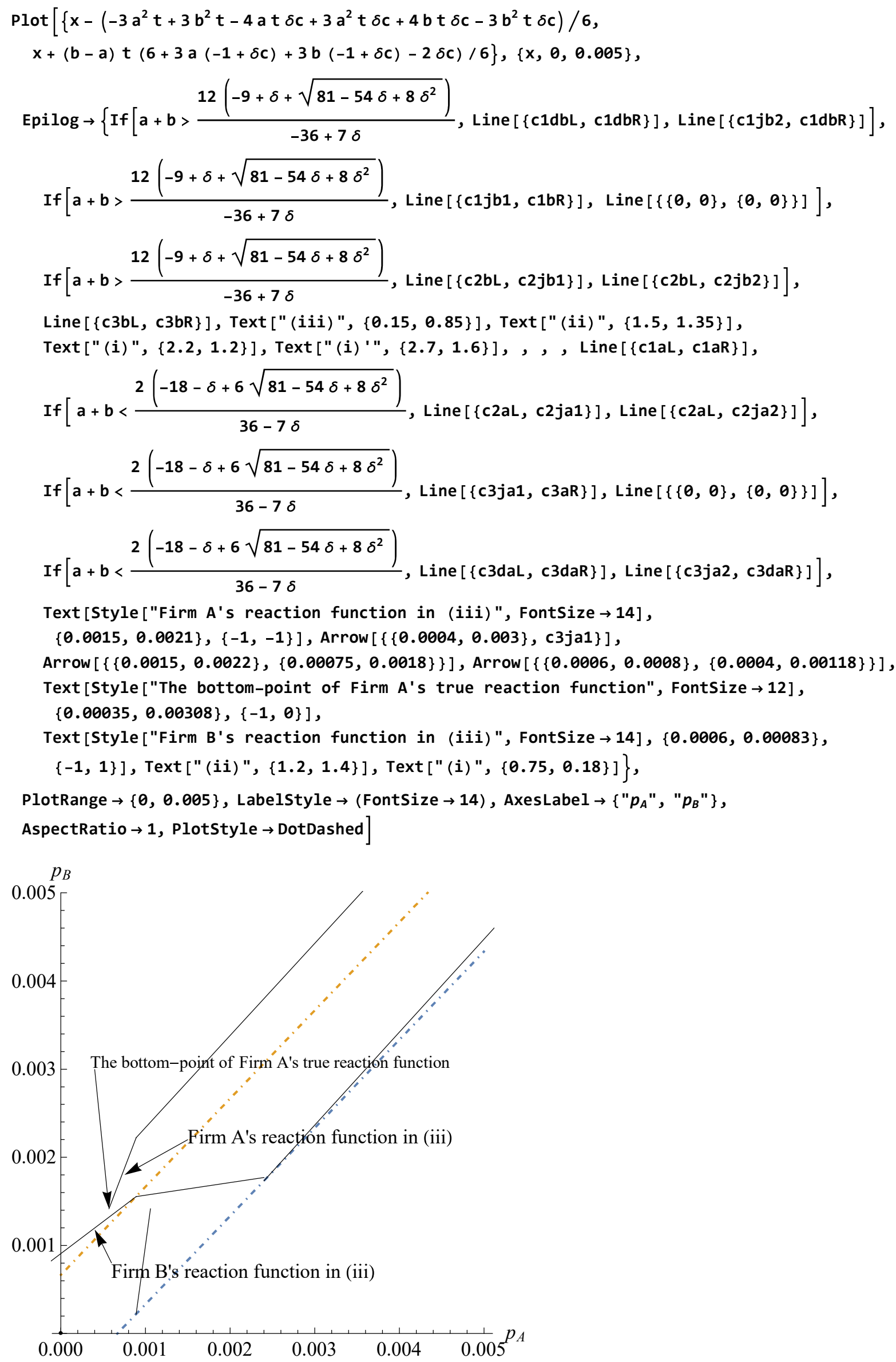
We now turn to the pricing equilibrium in the first period. First, we show that no equilibrium exists in (Case iii).

To this end, we show that the lowest point of Firm A's reaction function in (iii) is above Firm B's reaction function in (iii), as shown in the previous figure. If this property holds, Firm A's reaction function in (iii) never passes through the reaction function of Firm $B$ in (iii) because the slope of Firm A's reaction function in (iii) is steeper than that of Firm B in (iii).

Clear $[a, b, \delta, t, k]$

$\delta f=\delta$

$\delta \mathrm{c}=\delta$

$\delta$

$\delta$

The lowest point of Firm A's "true" reaction function in (iii) is point "c3jal" if if $a+b<\frac{2\left(-18-\delta+6 \sqrt{81-54 \delta+8 \delta^{2}}\right)}{36-7 \delta}$, otherwise, point "c3ja2". We show the two points in the following:

c3ja1

c3ja2

$$
\begin{aligned}
& \left\{\frac{1}{36}(-a+b) t\left(-2(9-5 \delta)+3(2+a+b)(9-5 \delta) \sqrt{\frac{9-2 \delta}{9-4 \delta}}-3(a+b)(3+\delta)\right),\right. \\
& \left.\frac{1}{18}(-a+b) t\left(-2-3 a-3 b+3(2+a+b) \sqrt{\frac{9-2 \delta}{9-4 \delta}}\right)(9-4 \delta)\right\} \\
& \left\{\frac{1}{9}(a-b) t\left(-18+a \delta+b \delta+2 \sqrt{\left(-8+a^{2}+2 b+b^{2}+2 a(1+b)\right) \delta(-9+2 \delta)}\right),\right. \\
& \left.\frac{1}{9}(-a+b) t(9(4-a-b)-(6-5 a-5 b) \delta-2 \sqrt{(2-a-b)(4+a+b)(9-2 \delta) \delta})\right\}
\end{aligned}
$$

The following is the reaction function of Firm B in (iii).

$$
\begin{aligned}
p b \rightarrow & \frac{(9-3 \delta c+10 \delta f) p a}{2(9-3 \delta c+5 \delta f)}+ \\
& ((b-a) t(2(3-\delta c)(3-\delta c+3 \delta f)-(a+b)((3-\delta c)(3-2 \delta c)+5(1-\delta c) \delta f))) / \\
& (2(9-3 \delta c+5 \delta f))
\end{aligned}
$$

We substitute $p_{A}$-element of point "c3jal" into the reaction function of Firm B in (iii).

$$
\begin{aligned}
& \text { Simplify }\left[\mathrm{pb} \rightarrow \frac{(9-3 \delta c+10 \delta f) p a}{2(9-3 \delta c+5 \delta f)}+\right. \\
& \quad((b-a) t(2(3-\delta c)(3-\delta c+3 \delta f)-(a+b)((3-\delta c)(3-2 \delta c)+5(1-\delta c) \delta f))) / \\
& (2(9-3 \delta c+5 \delta f)) / . p a \rightarrow \frac{1}{36}(-a+b) t \\
& \left.\left(-2(9-5 \delta)+3(2+a+b)(9-5 \delta) \sqrt{\frac{9-2 \delta}{9-4 \delta}}-3(a+b)(3+\delta)\right) / .\{\delta f \rightarrow \delta, \delta c \rightarrow \delta\}\right] \\
& p b \rightarrow \frac{1}{72(9+2 \delta)}(-a+b) t\left((9+7 \delta)\left(3(2+a+b)(9-5 \delta) \sqrt{\frac{9-2 \delta}{9-4 \delta}}-3(a+b)(3+\delta)+2(-9+5 \delta)\right)+\right. \\
& \left.36\left(18+6 \delta-4 \delta^{2}+a\left(-9+4 \delta+3 \delta^{2}\right)+b\left(-9+4 \delta+3 \delta^{2}\right)\right)\right)
\end{aligned}
$$


We check if this derived value of $p_{B}$ is smaller than $p_{B}$-element of point "c3jal". If the derived value of $p_{B}$ is actually smaller than $p_{B}$-element of point "c3jal", Firm A's "true" reaction function in (iii) does not pass through the reaction function of Firm B in (iii). The difference between the derived value of $p_{B}$ and the $p_{B}$-element of c3ja1:

$$
\begin{aligned}
& \text { Simplify }[\text { Factor }[ \\
& \frac{1}{72(9+2 \delta)}(-a+b) t\left((9+7 \delta)\left(3(2+a+b)(9-5 \delta) \sqrt{\frac{9-2 \delta}{9-4 \delta}}-3(a+b)(3+\delta)+2(-9+5 \delta)\right)+\right. \\
& \left.36\left(18+6 \delta-4 \delta^{2}+a\left(-9+4 \delta+3 \delta^{2}\right)+b\left(-9+4 \delta+3 \delta^{2}\right)\right)\right)- \\
& \left.\left.\frac{1}{18}(-a+b) t\left(-2-3 a-3 b+3(2+a+b) \sqrt{\frac{9-2 \delta}{9-4 \delta}}\right)(9-4 \delta)\right]\right] \\
& \frac{1}{24(9+2 \delta)}(a-b) t(-3+\delta)\left(3 a\left(21-27 \sqrt{\frac{9-2 \delta}{9-4 \delta}}+\delta+\sqrt{\frac{9-2 \delta}{9-4 \delta}} \delta\right)+\right. \\
& \left.3 b\left(21+\delta-27 \sqrt{\frac{-9+2 \delta}{-9+4 \delta}}+\delta \sqrt{\frac{-9+2 \delta}{-9+4 \delta}}\right)+2\left(63+23 \delta-81 \sqrt{\frac{-9+2 \delta}{-9+4 \delta}}+3 \delta \sqrt{\frac{-9+2 \delta}{-9+4 \delta}}\right)\right)
\end{aligned}
$$

We rearrange this value, and obtain the following:

$$
\begin{aligned}
& \frac{1}{24(9+2 \delta)}(b-a) t(3-\delta) \\
& \left(\left((2(63+23 \delta))-6(27-\delta) \sqrt{\frac{9-2 \delta}{9-4 \delta}}\right)-3(a+b)\left((27-\delta) \sqrt{\frac{9-2 \delta}{9-4 \delta}}-(21+\delta)\right)\right)
\end{aligned}
$$

We check the values of the first and the second terms in the largest parentheses

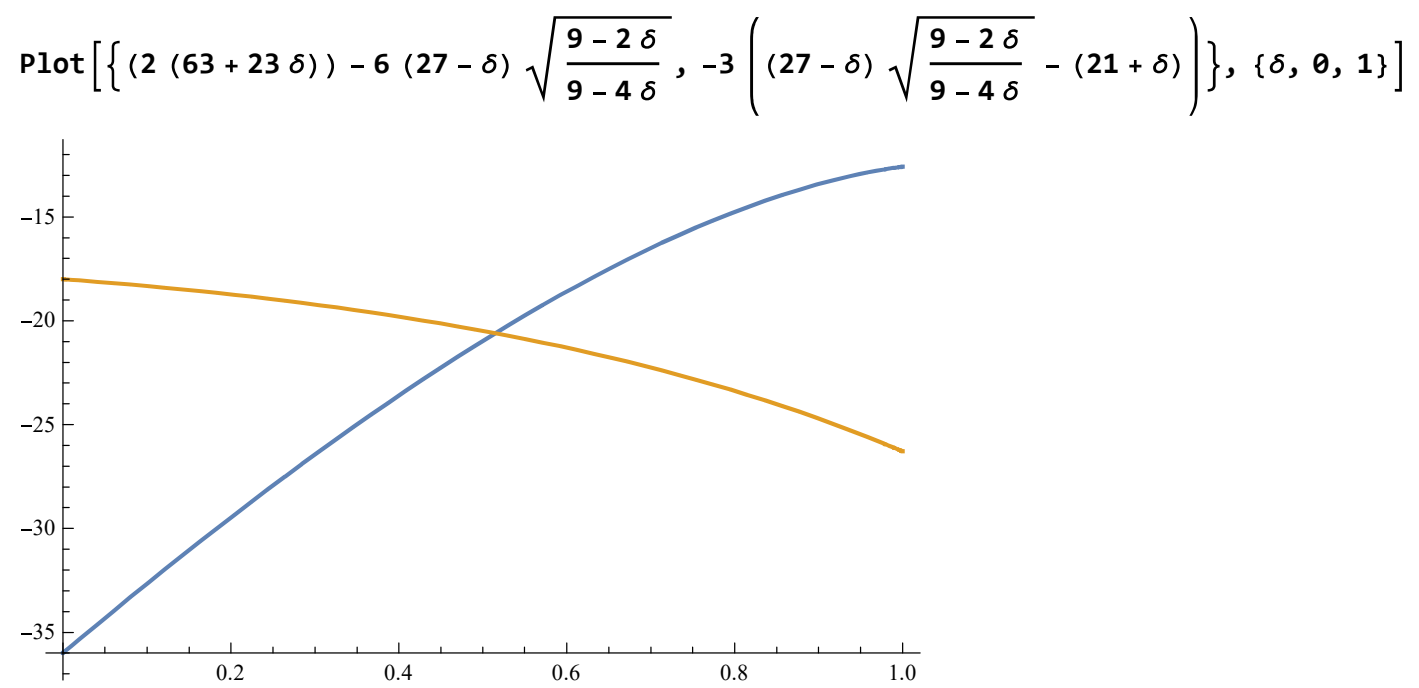

Both values are negative. Therefore, the difference between the derived value of $p_{B}$ and the $p_{B^{-}}$ element of c3ja1 is negative. That is, Firm A's "true" reaction function in (iii) does not pass through the reaction function of Firm $B$ in (iii) if $a+b<\frac{2\left(-18-\delta+6 \sqrt{81-54 \delta+8 \delta^{2}}\right)}{36-7 \delta}$.

We substitute $p_{A}$-element of $\mathrm{c} 3 \mathrm{ja} 2$ into the reaction function of Firm B in (iii). 


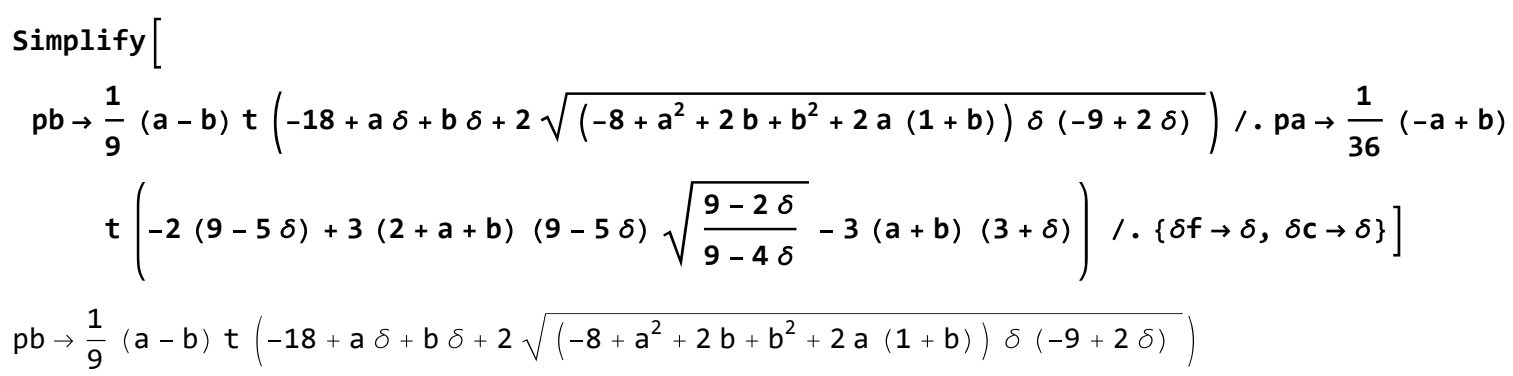

We check if this derived value of $p_{B}$ is smaller than $p_{B}$-element of point "c3ja2". If the derived value of $p_{B}$ is actually smaller than $p_{B}$-element of point "c3ja2", Firm A's "true" reaction function in (iii) does not pass through the reaction function of Firm $B$ in (iii). The difference between the derived value of $p_{B}$ and the $p_{B}$-element of $\mathrm{c} 3 \mathrm{ja} 2$ :

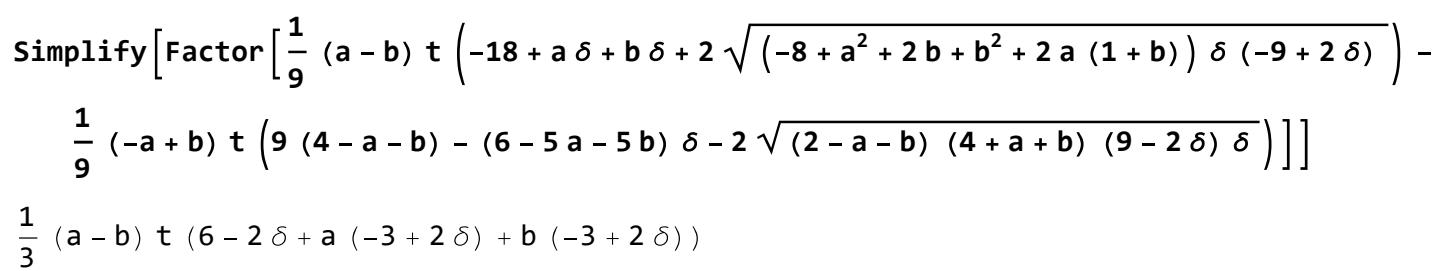

We rearrange this value, and obtain the following:

$-\frac{1}{3}(b-a) t(6-2 \delta+(a+b)(-3+2 \delta))$

The above is negative. The reason is as follows. $6-2 \delta+(\mathrm{a}+\mathrm{b})(-3+2 \delta)$ is decreasing in $(\mathrm{a}+\mathrm{b})$, hence it is minimized when $\mathrm{a}+\mathrm{b}=2$, and then $6-2 \delta+2(-3+2 \delta)=2 \delta>0$.

Therefore, the difference between the derived value of $p_{B}$ and the $p_{B}$-element of $\mathrm{c} 3 \mathrm{ja} 2$ is negative. That is, Firm A's "true" reaction function in (iii) does not pass through the reaction function of Firm $B$ in (iii) if $a+b \geq \frac{2\left(-18-\delta+6 \sqrt{81-54 \delta+8 \delta^{2}}\right)}{36-7 \delta}$.

Thus, for any $\mathrm{a}, \mathrm{b}$, and $\delta$, Firm A's "true" reaction function in (iii) does not pass through the reaction function of Firm B in (iii). This shows that the (pure-strategy) pricing equilibrium in period 1 does not exist in (Case iii).

Since (Case i) is symmetric to (Case iii), we can apply the same logic to conclude that the pricing equilibrium in the first period does not exist in (Case i).

We now turn to (Case ii), and identify conditions under which the pricing equilibrium in the first period exists in this case.

We pick up the reaction functions of the firms in (Case ii), without considering if they are in (Case ii). We derive the intersection between the reaction functions: 


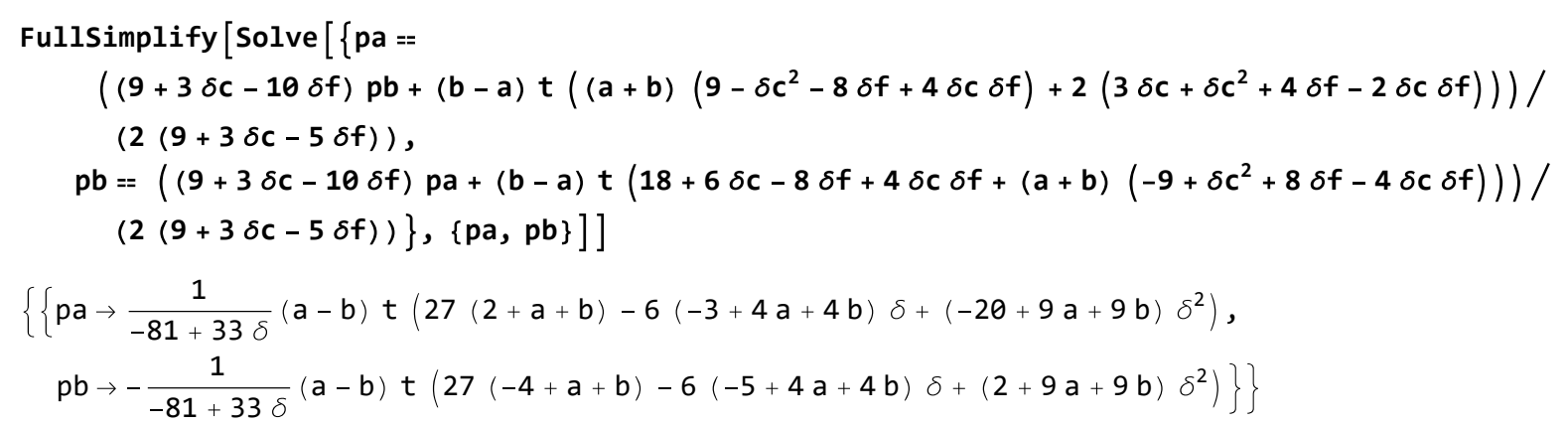

From the result, if there is an intersection between the reaction functions of Firms A and B in (Case ii), the equilibrium $p_{B}$ is the following:

Eq pb : $-\frac{1}{-81+33 \delta}(a-b) t\left(27(-4+a+b)-6(-5+4 a+4 b) \delta+(2+9 a+9 b) \delta^{2}\right)$

We can easily show that under the prices the realized $z$ satisfies $(a+b) / 4<z<(2+a+b) / 4$ (Case ii).

We rewrite the jump point of Firm A's reaction function (c3ja1 and c3ja2):

c3ja1

c3ja2

$$
\begin{aligned}
& \left\{\frac{1}{36}(-a+b) t\left(-2(9-5 \delta)+3(2+a+b)(9-5 \delta) \sqrt{\frac{9-2 \delta}{9-4 \delta}}-3(a+b)(3+\delta)\right),\right. \\
& \left.\frac{1}{18}(-a+b) t\left(-2-3 a-3 b+3(2+a+b) \sqrt{\frac{9-2 \delta}{9-4 \delta}}\right)(9-4 \delta)\right\} \\
& \left\{\frac{1}{9}(a-b) t\left(-18+a \delta+b \delta+2 \sqrt{\left(-8+a^{2}+2 b+b^{2}+2 a(1+b)\right) \delta(-9+2 \delta)}\right),\right. \\
& \left.\frac{1}{9}(-a+b) t(9(4-a-b)-(6-5 a-5 b) \delta-2 \sqrt{(2-a-b)(4+a+b)(9-2 \delta) \delta})\right\}
\end{aligned}
$$

If those values of $p_{B}$ in the two jump points are larger than the equilibrium $p_{B}$ in (Case ii), the equilibrium point is stable.

First, we compare $p_{B}$ at the intersection and at $\mathrm{c} 3 \mathrm{ja} 1$ :

$$
\begin{aligned}
& \text { Simplify }\left[\text { Factor } \left[\frac{1}{18}(-a+b) t\left(-2-3 a-3 b+3(2+a+b) \sqrt{\frac{9-2 \delta}{9-4 \delta}}\right)(9-4 \delta)-\right.\right. \\
& \left.\left.\left(-\frac{1}{-81+33 \delta}(a-b) t\left(27(-4+a+b)-6(-5+4 a+4 b) \delta+(2+9 a+9 b) \delta^{2}\right)\right)\right]\right] \\
& \frac{1}{-486+198 \delta}(a-b) t\left(3 a\left(27\left(-7+9 \sqrt{\frac{9-2 \delta}{9-4 \delta}}\right)-3\left(-53+69 \sqrt{\frac{9-2 \delta}{9-4 \delta}}\right) \delta+\left(-26+44 \sqrt{\frac{9-2 \delta}{9-4 \delta}}\right) \delta^{2}\right)+\right. \\
& 3 b\left(27\left(-7+9 \sqrt{\frac{9-2 \delta}{9-4 \delta}}\right)-3\left(-53+69 \sqrt{\frac{9-2 \delta}{9-4 \delta}}\right) \delta+\left(-26+44 \sqrt{\frac{9-2 \delta}{9-4 \delta}}\right) \delta^{2}\right)+ \\
& \left.2\left(81\left(-7+9 \sqrt{\frac{9-2 \delta}{9-4 \delta}}\right)+\left(297-621 \sqrt{\frac{9-2 \delta}{9-4 \delta}}\right) \delta+2\left(-19+66 \sqrt{\frac{9-2 \delta}{9-4 \delta}}\right) \delta^{2}\right)\right)
\end{aligned}
$$

Rearranging the above, we obtain

$$
\begin{aligned}
& \frac{1}{486-198 \delta}(b-a) t\left(\left(3\left(243-207 \delta+44 \delta^{2}\right) \sqrt{\frac{9-2 \delta}{9-4 \delta}}-(9-2 \delta)((63-39 \delta))\right)(a+b)-\right. \\
& \left.(9-2 \delta)(126-38 \delta)+6\left(243-207 \delta+44 \delta^{2}\right) \sqrt{\frac{9-2 \delta}{9-4 \delta}}\right)
\end{aligned}
$$


The sign of this value depends on $\delta$ and $\mathrm{g} \equiv \mathrm{a}+\mathrm{b}$.

We draw the area in which the above value is negative, that is, the equilibrium point in (Case ii) is not stable.

(Horizontal axis is $\delta$, Vertical axis is $\mathrm{g}=\mathrm{a}+\mathrm{b}$ ):

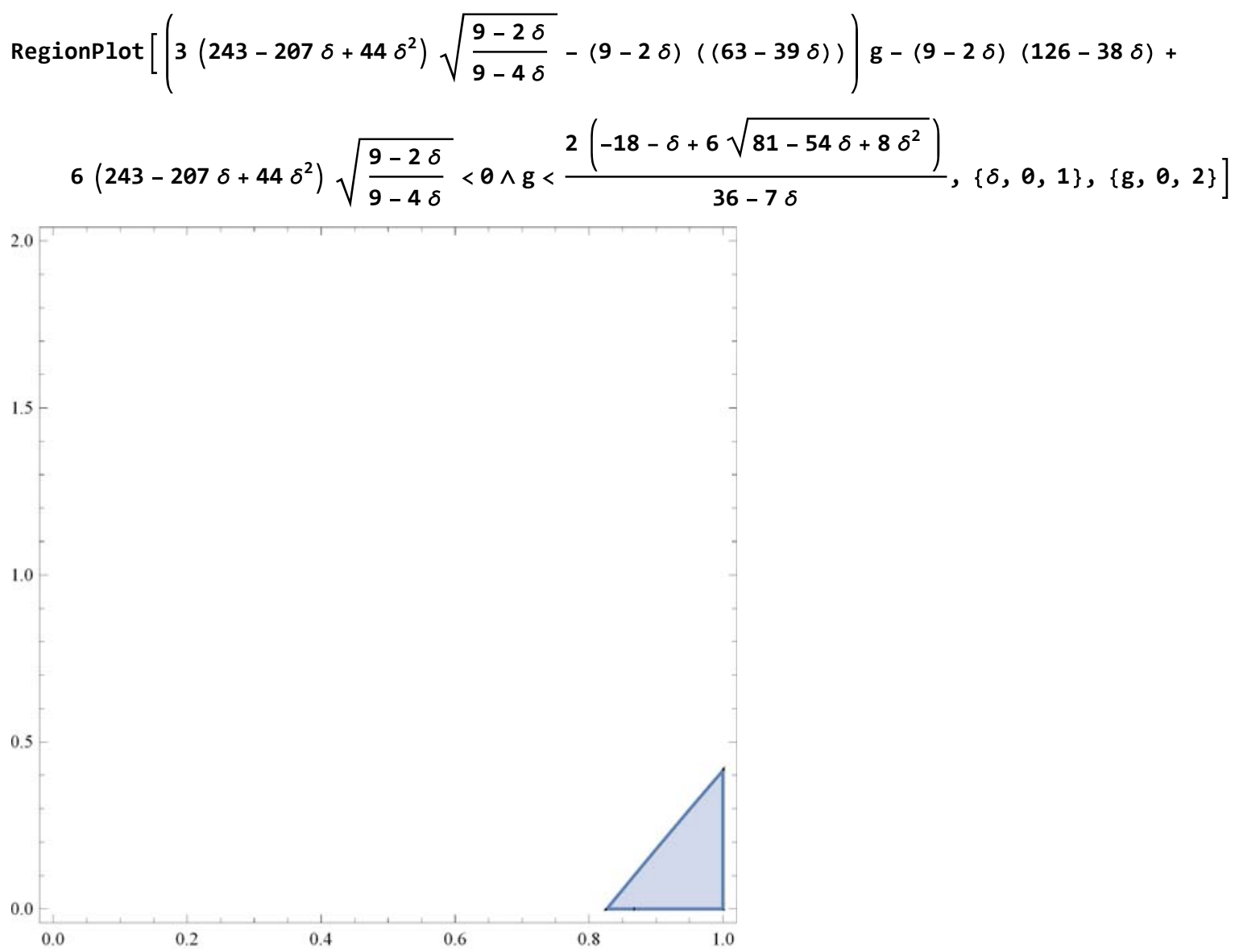

On the blue area,the equilibrium point in (Case ii) is not stable.

From the figure, we can find the highest value of $\delta$ in which the equilibrium point in (Case ii) is stable for any $\mathrm{g}=\mathrm{a}+\mathrm{b}$, by solving the following equation with respect to $\delta$.

NSolve $\left[-(9-2 \delta)(126-38 \delta)+6\left(243-207 \delta+44 \delta^{2}\right) \sqrt{\frac{9-2 \delta}{9-4 \delta}}=0, \delta\right]$

$\{\{\delta \rightarrow 4.5\},\{\delta \rightarrow 0.826528\}\}$

We find that if $\delta<0.826$, the equilibrium point in area (ii) is stable for any $\mathrm{g}=\mathrm{a}+\mathrm{b}$.

From the figure, we can find the lowest value of $g$ in which the equilibrium point in area (ii) is stable for any $\delta$, by solving the following equation with respect to $g$.

$$
\begin{aligned}
& \text { Solve }\left[\left(3\left(243-207 \delta+44 \delta^{2}\right) \sqrt{\frac{9-2 \delta}{9-4 \delta}}-(9-2 \delta)((63-39 \delta))\right) \mathrm{g}-\right. \\
& \left.\quad(9-2 \delta)(126-38 \delta)+6\left(243-207 \delta+44 \delta^{2}\right) \sqrt{\frac{9-2 \delta}{9-4 \delta}}=0 / . \delta \rightarrow 1, \mathrm{~g}\right] \\
& \left\{\left\{\mathrm{g} \rightarrow \frac{77-12 \sqrt{35}}{3(-7+2 \sqrt{35})}\right\}\right\}
\end{aligned}
$$


N [\%]

$\{\{g \rightarrow 0.414379\}\}$

Because of symmetry, the result can be applied to the highest value of $g$ in which the equilibrium point in area (ii) is stable for any $\delta$.

We derive the threshold $g$ as a function of $\delta$.

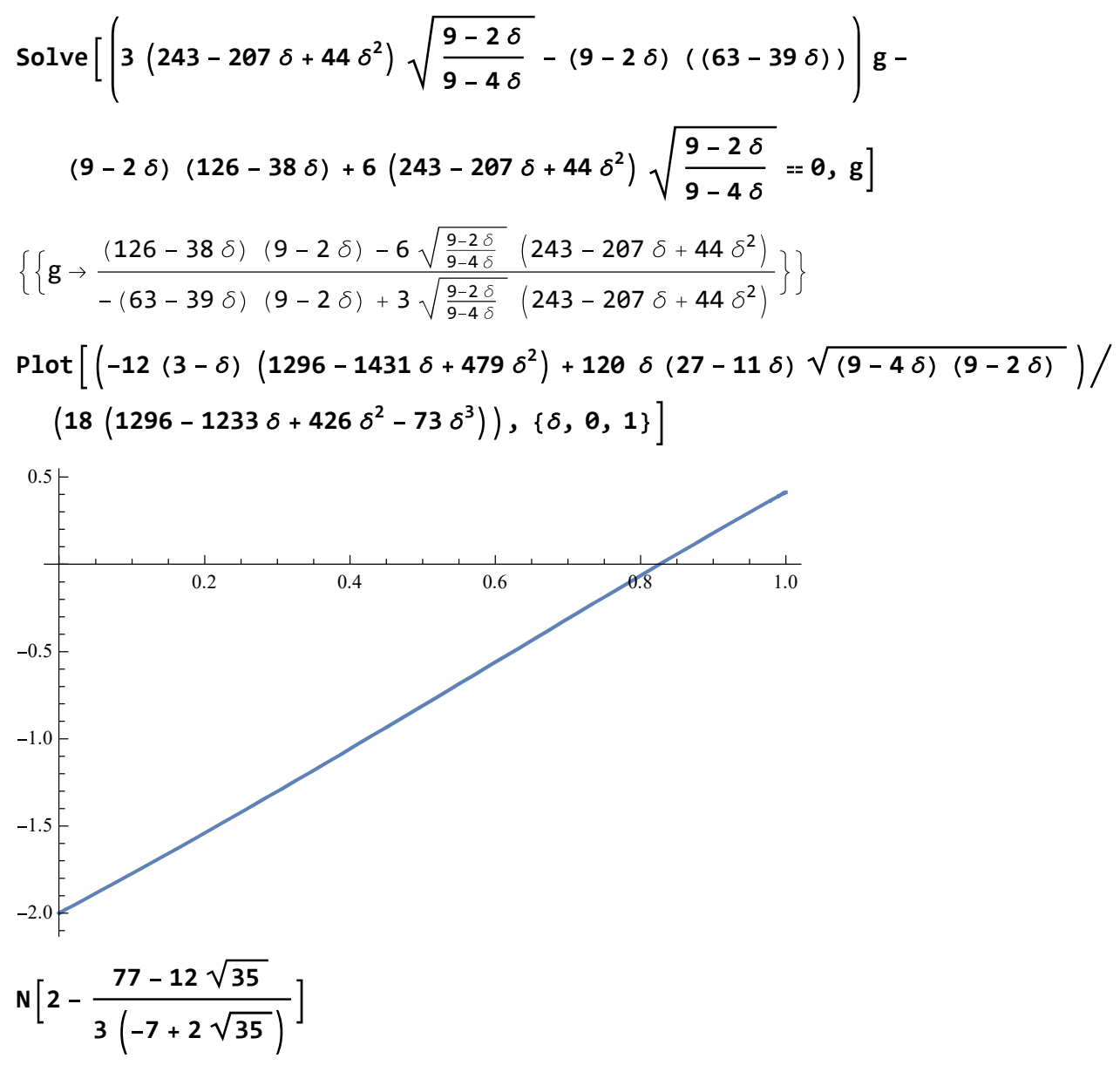

1.58562

Thus, we find that if $0.415<\mathrm{g}<1.585$, the equilibrium point in area (ii) is stable for any $\delta$.

$$
\begin{aligned}
& \left\{\frac{1}{9}(a-b) t\left(-18+a \delta+b \delta+2 \sqrt{\left(-8+a^{2}+2 b+b^{2}+2 a(1+b)\right) \delta(-9+2 \delta)}\right),\right. \\
& \left.\frac{1}{9}(-a+b) t(9(4-a-b)-(6-5 a-5 b) \delta-2 \sqrt{(2-a-b)(4+a+b)(9-2 \delta) \delta})\right\}
\end{aligned}
$$

Second, we compare $p_{B}$ at the intersection and at c3ja2: 


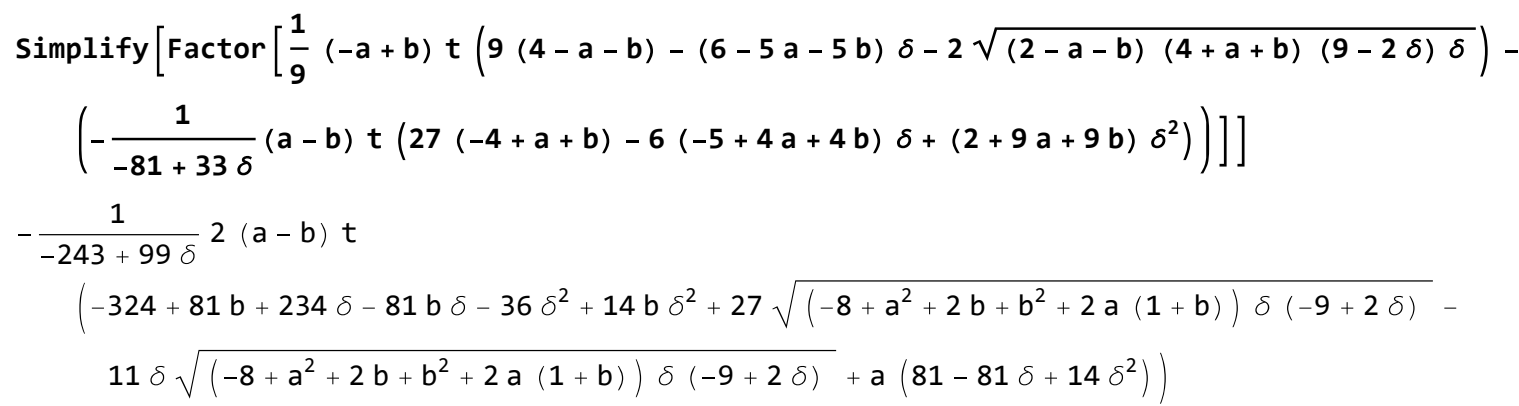

Rearranging the above, we obtain

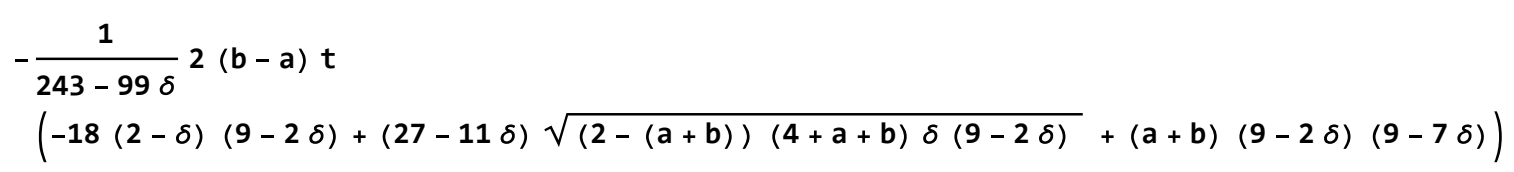

The sign of this value depends on $\delta$ and $\mathrm{g}=\mathrm{a}+\mathrm{b}$.

We draw the area in which the above value is negative, that is, the equilibrium point in (Case ii) is not stable.

(Horizontal axis is $\delta$, Vertical axis is $\mathrm{g}=\mathrm{a}+\mathrm{b}$ ):
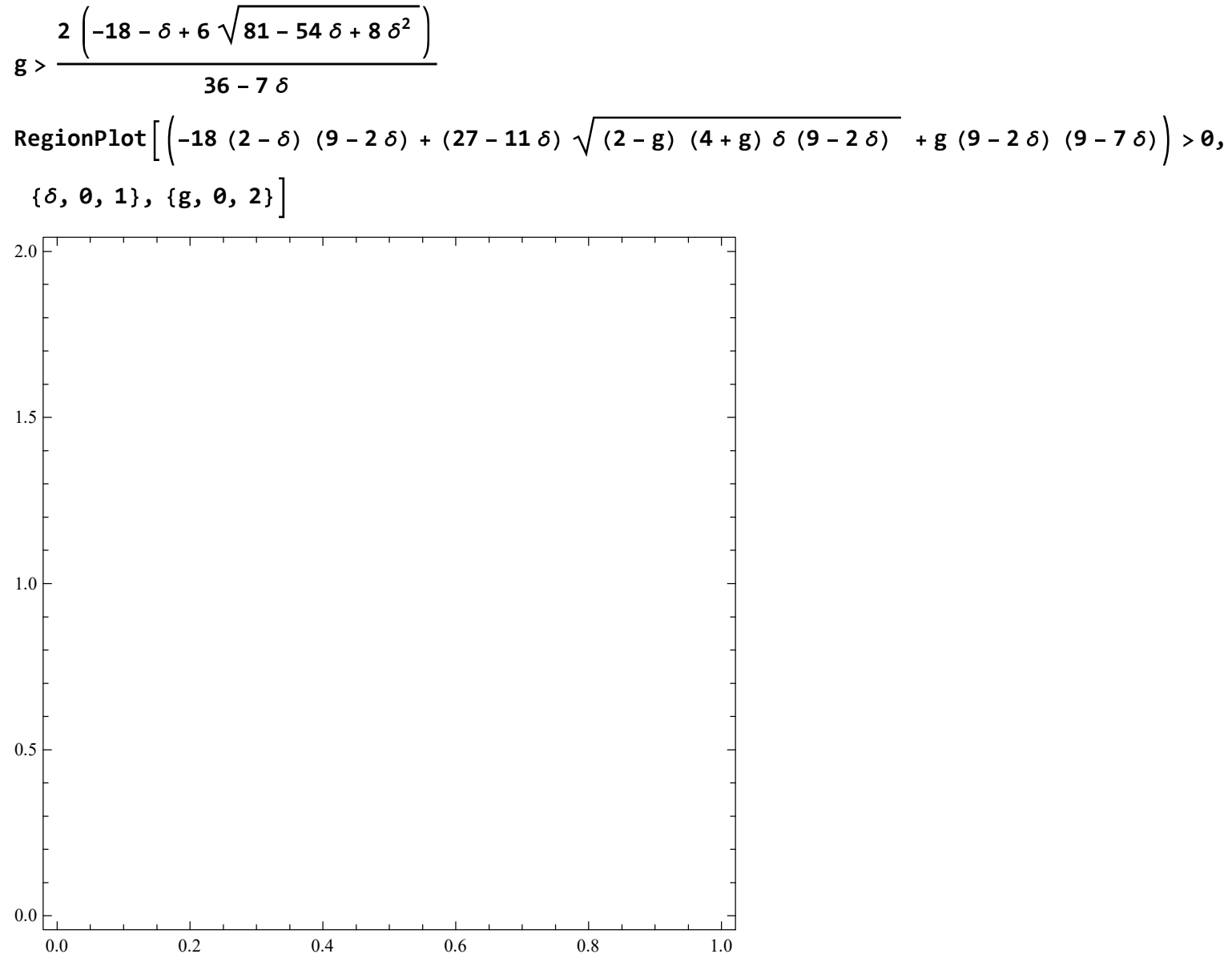

This means that Firm B's deviation incentive does not matter.

From the two arguments, we find that if $0.415<\mathrm{g}<1.585$, the equilibrium point in area (ii) is stable for any $\delta$.

Thus we conclude that (i) if $\delta<0.826$, the pricing equilibrium exists in (Case ii) for any a $+\mathrm{b}$, and (ii) if $0.415<\mathrm{a}+\mathrm{b}<1.585$, then the pricing equilibrium exists in (Case ii) for any $\delta$. 


\section{First Period - Locations}

The profits of Firms A and B are respectively

$$
\begin{aligned}
& \text { Simplify [Factor }\left[p a z+\delta f \frac{1}{9}(b-a) t\left(2+2 a+a^{2}+2 b+2 a b+b^{2}-8 z-2 a z-2 b z+10 z^{2}\right) /\right. \\
& z \rightarrow \frac{3(p b-p a)}{2(b-a) t(3+\delta c)}+\frac{((a+b)(3-\delta c)+2 \delta c)}{2(3+\delta c)} / .\{p a \rightarrow((a-b) t \\
& \left.\left(-27(2+a+b)-54 \delta c+3(-4+a+b) \delta c^{2}-4(-9+3 a(-2+\delta c)+3 b(-2+\delta c)-8 \delta c) \delta f\right)\right) / \\
& (81+27 \delta \mathrm{c}-60 \delta f), \mathrm{pb} \rightarrow-\left(\left(( \mathrm { a } - \mathrm { b } ) \mathrm { t } \left(-27(-4+\mathrm{a}+\mathrm{b})+54 \delta \mathrm{c}+3(2+\mathrm{a}+\mathrm{b}) \delta \mathrm{c}^{2}-\right.\right.\right. \\
& 4(21+3 a(-2+\delta c)+3 b(-2+\delta c)+2 \delta c) \delta f)) /(81+27 \delta c-60 \delta f))\}]] \\
& \text { Simplify }\left[\text { Factor } \left[p b(1-z)+\delta f \frac{1}{9}(b-a) t\left(8-4 a+a^{2}-4 b+2 a b+b^{2}-8 z-2 a z-2 b z+10 z^{2}\right) / .\right.\right. \\
& z \rightarrow \frac{3(p b-p a)}{2(b-a) t(3+\delta c)}+\frac{((a+b)(3-\delta c)+2 \delta c)}{2(3+\delta c)} / .\{p a \rightarrow((a-b) t \\
& \left.\left(-27(2+a+b)-54 \delta c+3(-4+a+b) \delta c^{2}-4(-9+3 a(-2+\delta c)+3 b(-2+\delta c)-8 \delta c) \delta f\right)\right) / \\
& (81+27 \delta \mathrm{c}-60 \delta f), \mathrm{pb} \rightarrow-\left(\left(( \mathrm { a } - \mathrm { b } ) \mathrm { t } \left(-27(-4+\mathrm{a}+\mathrm{b})+54 \delta \mathrm{c}+3(2+\mathrm{a}+\mathrm{b}) \delta \mathrm{c}^{2}-\right.\right.\right. \\
& 4(21+3 a(-2+\delta c)+3 b(-2+\delta c)+2 \delta c) \delta f)) /(81+27 \delta c-60 \delta f))\}]] \\
& -\frac{1}{18(27+9 \delta c-20 \delta f)^{2}} \\
& (a-b) \text { t }\left(9 a^{2}\left(81+3 \delta c^{3}+45 \delta f-184 \delta f^{2}+80 \delta f^{3}+3 \delta c^{2}(-3+7 \delta f)+\delta c\left(-27+138 \delta f-88 \delta f^{2}\right)\right)+\right. \\
& 9 b^{2}\left(81+3 \delta c^{3}+45 \delta f-184 \delta f^{2}+80 \delta f^{3}+3 \delta c^{2}(-3+7 \delta f)+\delta c\left(-27+138 \delta f-88 \delta f^{2}\right)\right)- \\
& 12 b\left(-243+18 \delta c^{3}+\delta c^{2}(27-57 \delta f)+216 \delta f+84 \delta f^{2}-80 \delta f^{3}+\delta c\left(-162-9 \delta f+88 \delta f^{2}\right)\right)+ \\
& 4\left(729+108 \delta c^{3}-972 \delta f+216 \delta f^{2}+80 \delta f^{3}-9 \delta c^{2}(-72+43 \delta f)+3 \delta c\left(405-450 \delta f+104 \delta f^{2}\right)\right)+ \\
& 6 a\left(3 b\left(81+3 \delta c^{3}+45 \delta f-184 \delta f^{2}+80 \delta f^{3}+3 \delta c^{2}(-3+7 \delta f)+\delta c\left(-27+138 \delta f-88 \delta f^{2}\right)\right)-\right. \\
& \left.\left.2\left(-243+18 \delta c^{3}+\delta c^{2}(27-57 \delta f)+216 \delta f+84 \delta f^{2}-80 \delta f^{3}+\delta c\left(-162-9 \delta f+88 \delta f^{2}\right)\right)\right)\right) \\
& -\frac{1}{18(27+9 \delta c-20 \delta f)^{2}} \\
& (a-b) t\left(9 a^{2}\left(81+3 \delta c^{3}+45 \delta f-184 \delta f^{2}+80 \delta f^{3}+3 \delta c^{2}(-3+7 \delta f)+\delta c\left(-27+138 \delta f-88 \delta f^{2}\right)\right)+\right. \\
& 9 b^{2}\left(81+3 \delta c^{3}+45 \delta f-184 \delta f^{2}+80 \delta f^{3}+3 \delta c^{2}(-3+7 \delta f)+\delta c\left(-27+138 \delta f-88 \delta f^{2}\right)\right)+ \\
& 4\left(2916+27 \delta c^{3}-1863 \delta f-1944 \delta f^{2}+1280 \delta f^{3}+9 \delta c^{2}(45+16 \delta f)-18 \delta c\left(-108+3 \delta f+56 \delta f^{2}\right)\right)+ \\
& 12 b\left(-486+9 \delta c^{3}+81 \delta f+636 \delta f^{2}-320 \delta f^{3}-6 \delta c^{2}(-9+20 \delta f)+\delta c\left(-81-423 \delta f+352 \delta f^{2}\right)\right)+ \\
& 6 a\left(3 b\left(81+3 \delta c^{3}+45 \delta f-184 \delta f^{2}+80 \delta f^{3}+3 \delta c^{2}(-3+7 \delta f)+\delta c\left(-27+138 \delta f-88 \delta f^{2}\right)\right)+\right. \\
& \left.\left.2\left(-486+9 \delta c^{3}+81 \delta f+636 \delta f^{2}-320 \delta f^{3}-6 \delta c^{2}(-9+20 \delta f)+\delta c\left(-81-423 \delta f+352 \delta f^{2}\right)\right)\right)\right)
\end{aligned}
$$

Rearranging them, we obtain the profits of Firms A and B respectively

$$
\begin{aligned}
& \frac{(b-a) t}{18(27+9 \delta c-20 \delta f)^{2}} \\
& \left(9(a+b)^{2}\left(3 \delta c^{3}-3 \delta c^{2}(3-7 \delta f)-\delta c\left(27-138 \delta f+88 \delta f^{2}\right)+81+45 \delta f-184 \delta f^{2}+80 \delta f^{3}\right)+\right. \\
& 12(a+b)\left(-18 \delta c^{3}-3 \delta c^{2}(9-19 \delta f)+\delta c\left(162+9 \delta f-88 \delta f^{2}\right)+243-216 \delta f-84 \delta f^{2}+80 \delta f^{3}\right)+ \\
& \left.4\left(108 \delta c^{3}+9 \delta c^{2}(72-43 \delta f)+3 \delta c\left(405-450 \delta f+104 \delta f^{2}\right)+729-972 \delta f+216 \delta f^{2}+80 \delta f^{3}\right)\right) \\
& \frac{1}{18(27+9 \delta c-20 \delta f)^{2}}(b-a) t \\
& \left(9(a+b)^{2}\left(3 \delta c^{3}-3 \delta c^{2}(3-7 \delta f)-\delta c\left(27-138 \delta f+88 \delta f^{2}\right)+81+45 \delta f-184 \delta f^{2}+80 \delta f^{3}\right)+\right. \\
& 12(a+b)\left(9 \delta c^{3}+6 \delta c^{2}(9-20 \delta f)-\delta c\left(81+423 \delta f-352 \delta f^{2}\right)-486+81 \delta f+636 \delta f^{2}-320 \delta f^{3}\right)+ \\
& \left.4\left(27 \delta c^{3}+9 \delta c^{2}(45+16 \delta f)+18 \delta c\left(108-3 \delta f-56 \delta f^{2}\right)+2916-1863 \delta f-1944 \delta f^{2}+1280 \delta f^{3}\right)\right)
\end{aligned}
$$

We define J1, the coefficient of $(a+b)^{2}$, as follows: 
$J 1=\left(3 \delta c^{3}-3 \delta c^{2}(3-7 \delta f)-\delta c\left(27-138 \delta f+88 \delta f^{2}\right)+81+45 \delta f-184 \delta f^{2}+80 \delta f^{3}\right)$
$81+3 \delta c^{3}-3 \delta c^{2}(3-7 \delta f)+45 \delta f-184 \delta f^{2}+80 \delta f^{3}-\delta c\left(27-138 \delta f+88 \delta f^{2}\right)$

Differentiating Firm A's profit with respect to $a$ and Firm B's profit with respect to $b$, we obtain

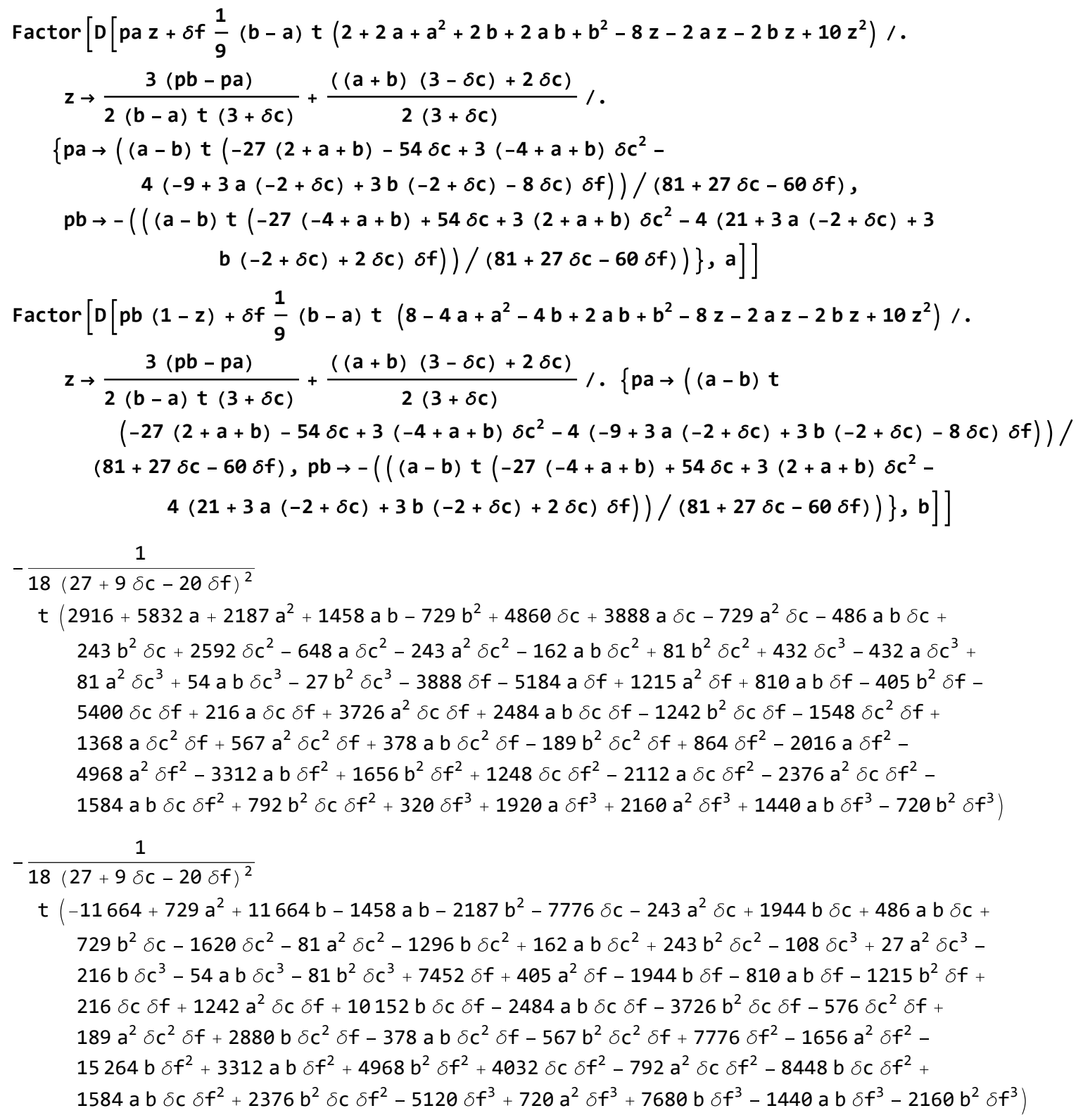

The above first-order derivatives can be rearranged as follows.

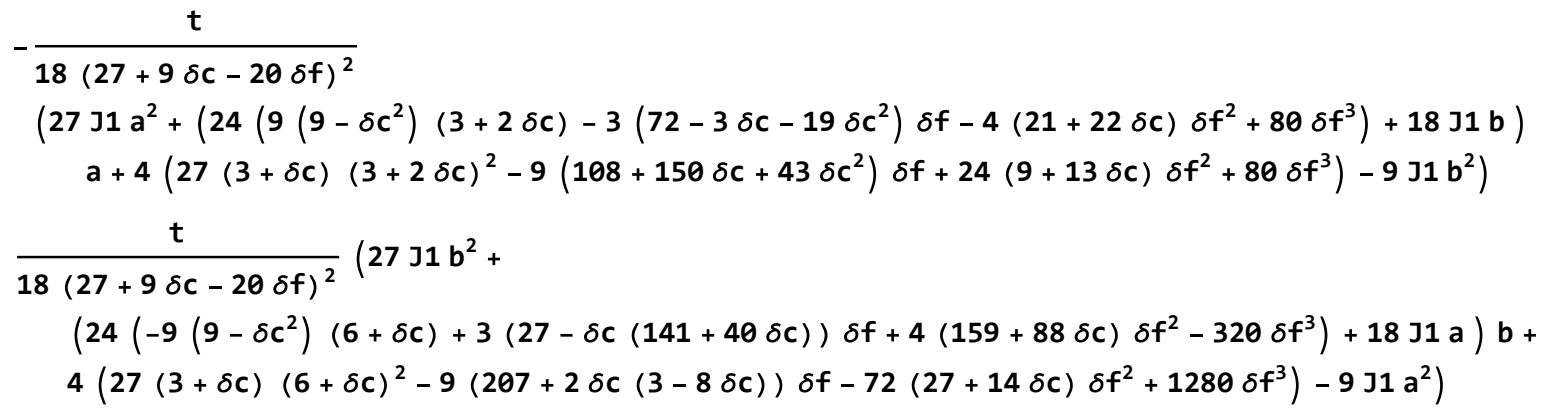

First, we show that the derivative of Firm A's profit monotonically decreases in $a$. This implies that Firm A's optimal location choice is $a=0$. To show this, we show that the coefficients to $a^{2}$, 
$a$ and the constant term in Firm A's first-order derivative are all positive. For the coefficients to $a^{2}$ and $a$, see the following figures, which show they are both positive.

Plot3D $[J 1,\{\delta c, 0,1\},\{\delta f, \theta, 1\}$, PlotRange $\rightarrow\{0,1\}]$

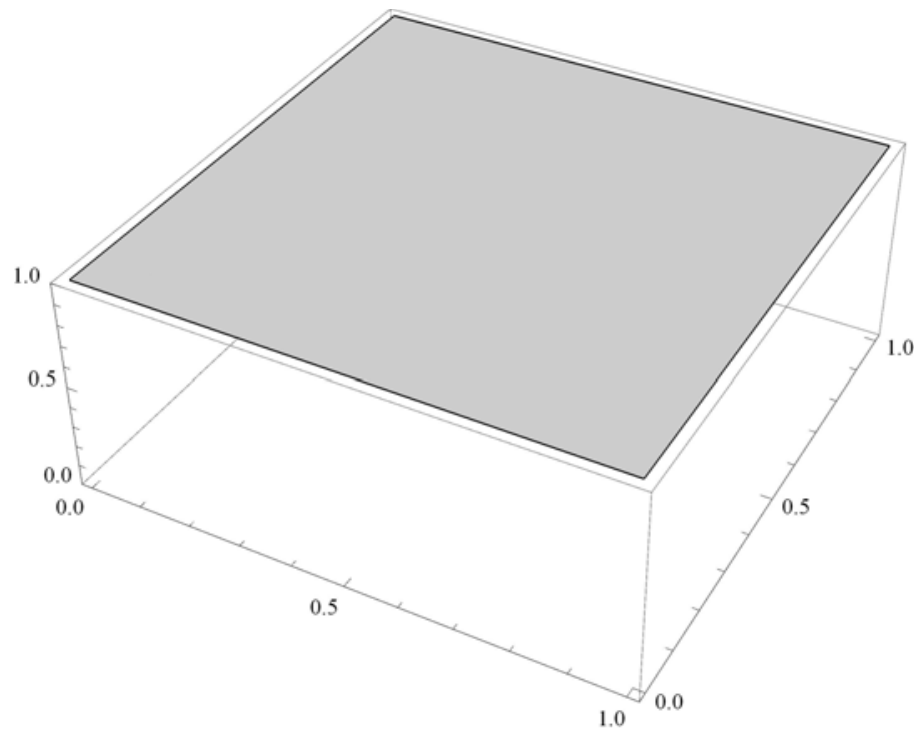

Plot3D $\left[24\left(9\left(9-\delta c^{2}\right)(3+2 \delta c)-3\left(72-3 \delta c-19 \delta c^{2}\right) \delta f-4(21+22 \delta c) \delta f^{2}+80 \delta f^{3}\right)\right.$, $\{\delta c, 0,1\},\{\delta f, 0,1\}$, PlotRange $\rightarrow\{0,1\}]$

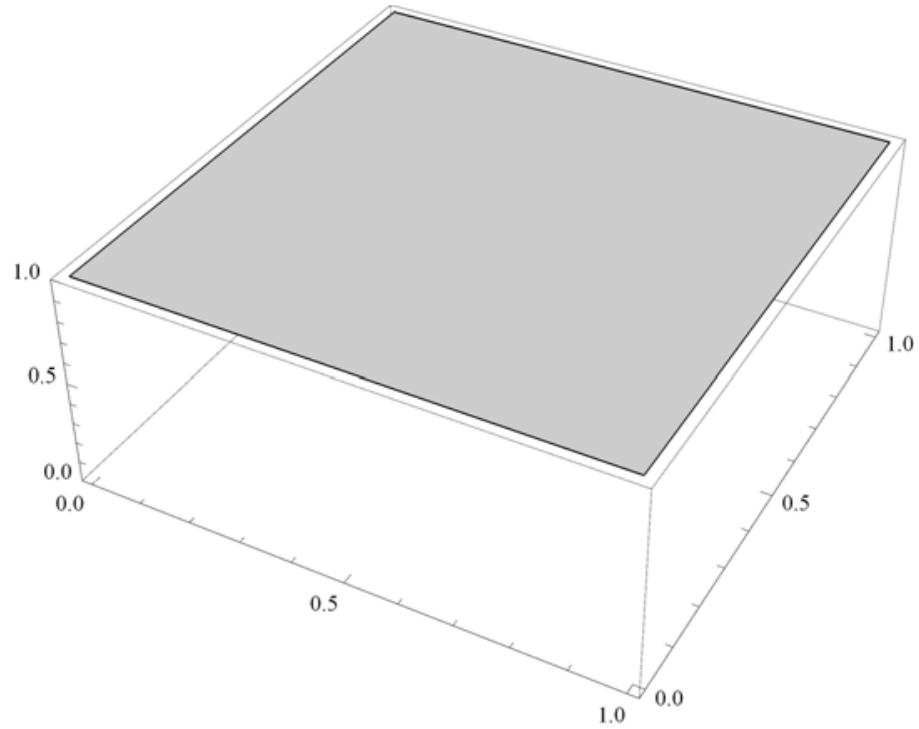

Those are positive.

For the constant term in Firm A's first-order derivative, notice that it is minimized when $b=1$. As the next figure shows, the constant term is positive when $b=1$. 


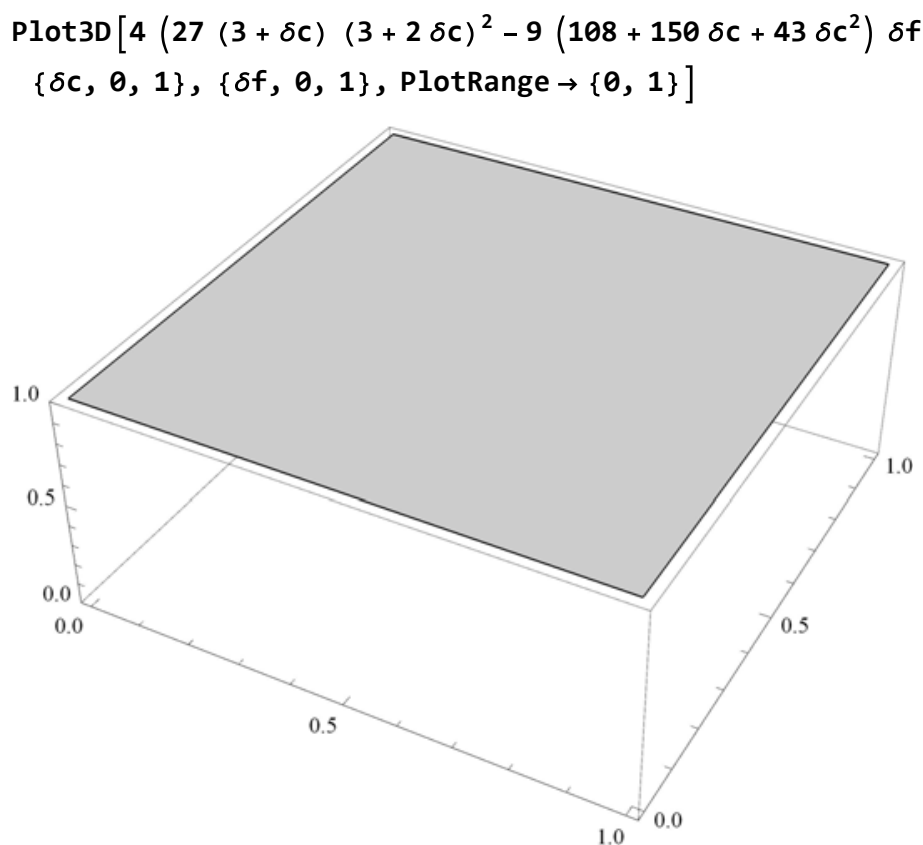

Put together, we have shown that Firm A's profit decreases monotonically in $a$. Thus $a=0$ is Firm A's optimal location choice.

Next, we turn to Firm B's problem. Differentiating the numerator of the first-order derivative of Firm B's profit with respect to $b$, we obtain

$54 \mathrm{~J} 1 \mathrm{~b}+$

$\left(24\left(-9\left(9-\delta c^{2}\right)(6+\delta c)+3(27-\delta c(141+40 \delta c)) \delta f+4(159+88 \delta c) \delta f^{2}-320 \delta f^{3}\right)+18 J 1 a\right)$

This is maximized when $a=b=1$. At $a=b=1$, we can show that this is negative (see the following figure).

Plot3D [

$54 J 1+\left(24\left(-9\left(9-\delta c^{2}\right)(6+\delta c)+3(27-\delta c(141+40 \delta c)) \delta f+4(159+88 \delta c) \delta f^{2}-320 \delta f^{3}\right)+18 J 1\right)$, $\{\delta c, 0,1\},\{\delta f, 0,1\}$, PlotRange $\rightarrow\{-1,0\}]$

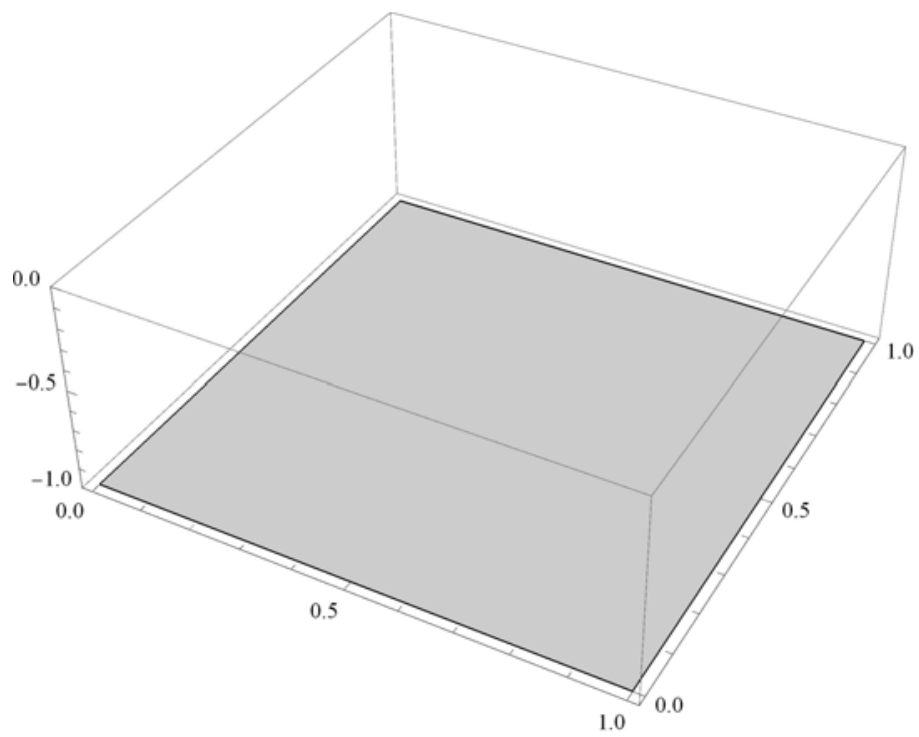

Thus the first-order derivative of Firm B's profit decreases monotonically in $b$.

We now show that at $a=0$ and $b=1$, the numerator of the first-order derivative of Firm B's profit is positive (see the following figure). This implies that Firm B's optimal location choice is $b=1$. 


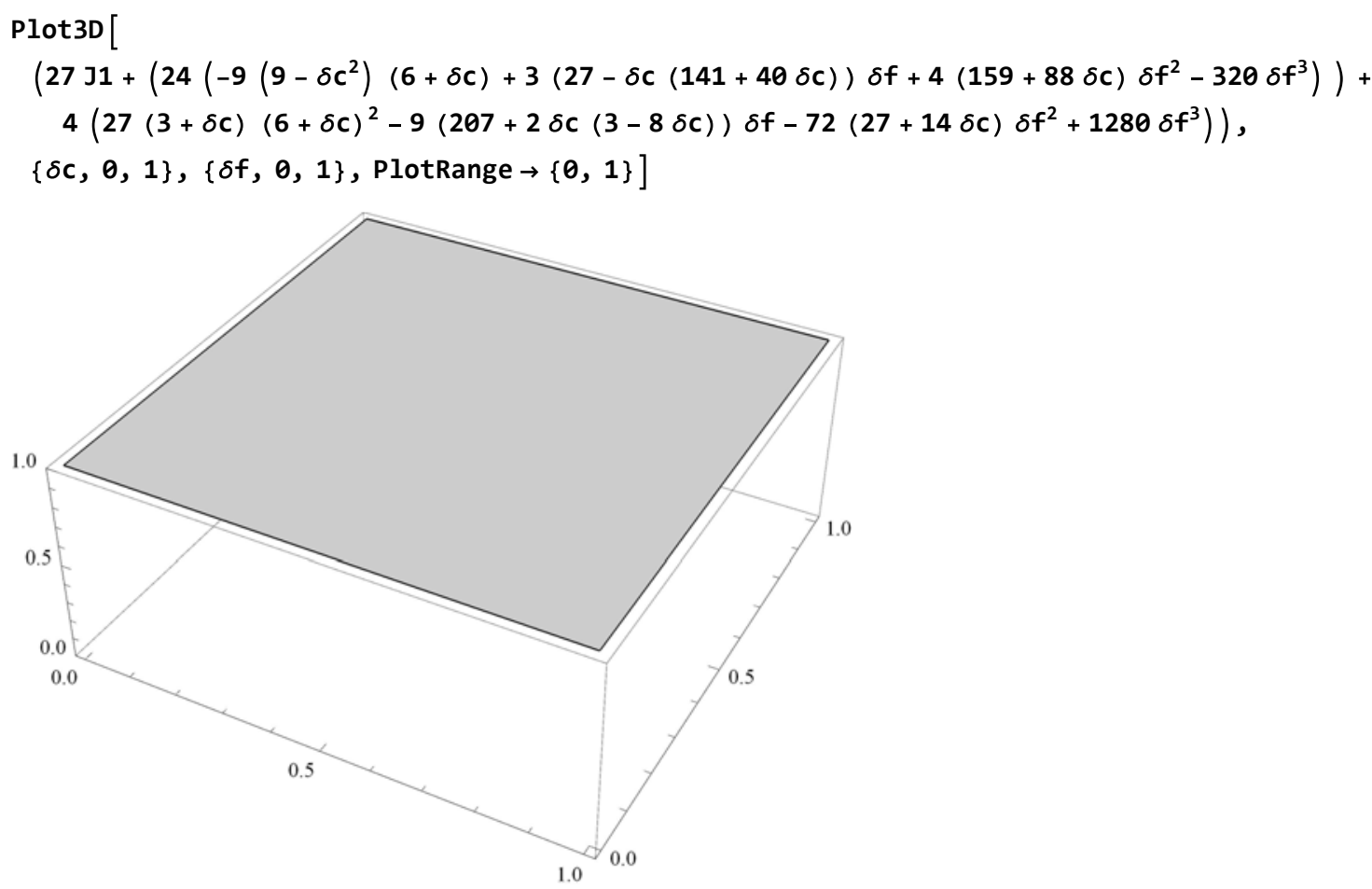

Therefore, the equilibrium locations are $a=0, b=1$.

The rest of Proposition 1 follows by substituting $a=0, b=1$ into relevant prices and the locations of marginal consumers in the two periods.

\section{Proofs of Propositions 2 and 3.}

Because each firm's reaction function consists of three different pieces, we need to derive the 'true' reaction function by checking when the firm's profit obtains a global, rather than, local maximum.

From here on, we assume that firms' discount factor is $\delta$ and consumers' discount factor is 0 , as stated in Propositions 2 and 3.

$\delta f=\delta$

$\delta$

$\delta c=0$

0

From Firm A's reaction function derived in the proof of Proposition 1, we check the endpoints of each of the three line segments corresponding to the three cases: (i) $0 \leq \mathrm{z} \leq(\mathrm{a}+\mathrm{b}) / 4$, (ii) $(\mathrm{a}+\mathrm{b}) / 4<\mathrm{z}<(2+\mathrm{a}+\mathrm{b}) / 4$, (iii) $(2+\mathrm{a}+\mathrm{b}) / 4 \leq \mathrm{z} \leq 1$.

(Case i): The first endpoint below locates the left-hand side of Firm A's reaction function, and the second endpoint below locates the right-hand side of Firm A's reaction function in the first period. 


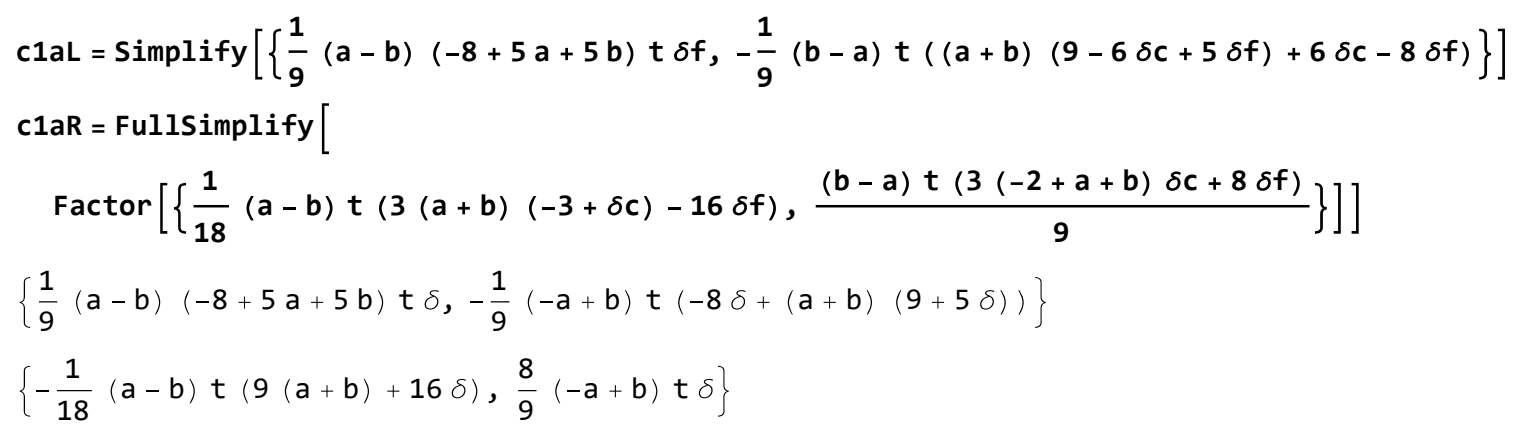

(Case ii): The first endpoint below locates the left-hand side of Firm A's reaction function, and the second endpoint below locates the right-hand side of Firm A's reaction function in the first period.

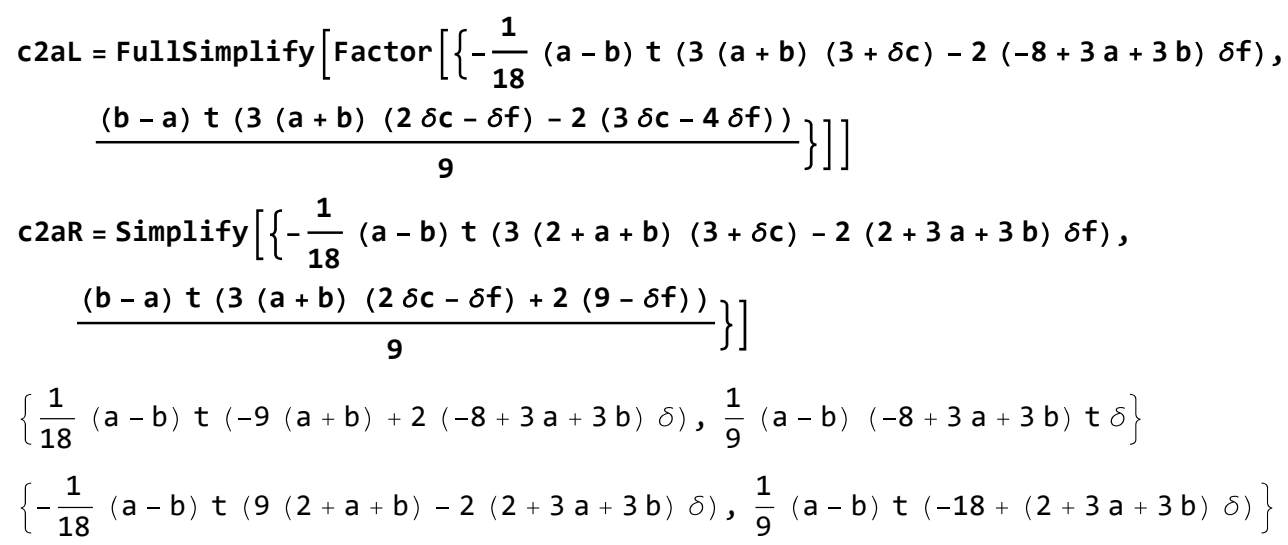

At this stage, we cannot say if Firm A's reaction function is also continuous in (Case i) and (Case ii). We will come back to this shortly. Note that the left-hand endpoint in (Case i) corresponds to $(\mathrm{a}+\mathrm{b}) / 4=\mathrm{z}$.

(Case iii): The first endpoint below locates the left-hand side of Firm A's reaction function, and the second endpoint below locates the right-hand side of Firm A's reaction function in in the first period.

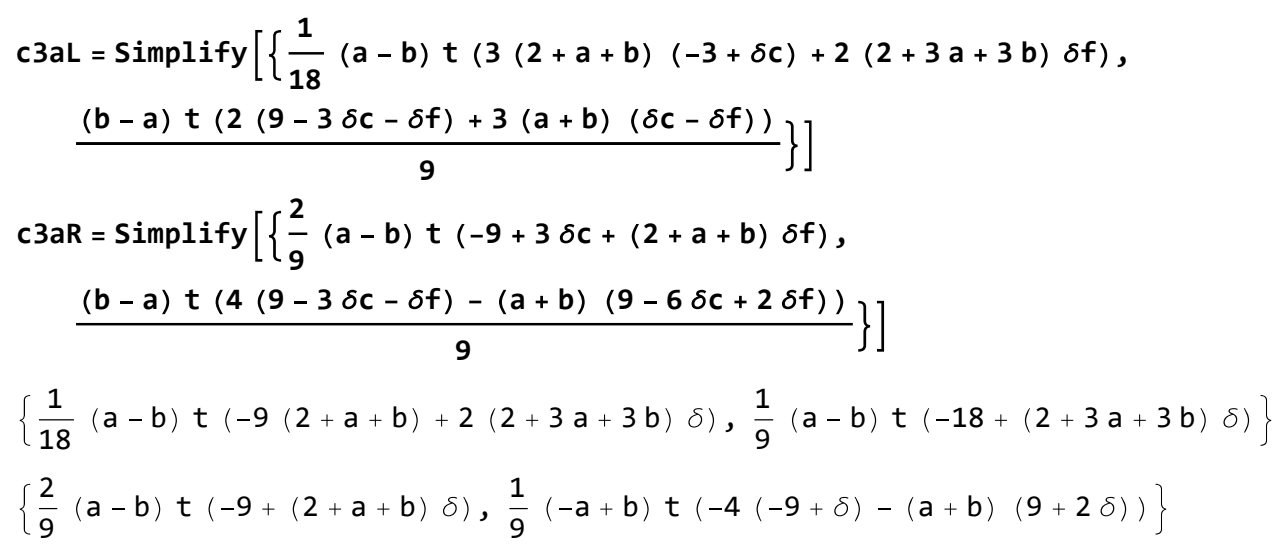

As shown above, the left endpoint of Firm A's reaction function in (Case iii) coincides with the right endpoint of Firm A's reaction function in (Case ii). That is, Firm A's reaction function is continuous in (Case ii) and (Case iii).

(Case iii)': When $\mathrm{z}=1$, the reaction function of Firm A consists of the segment connecting the following two points. 


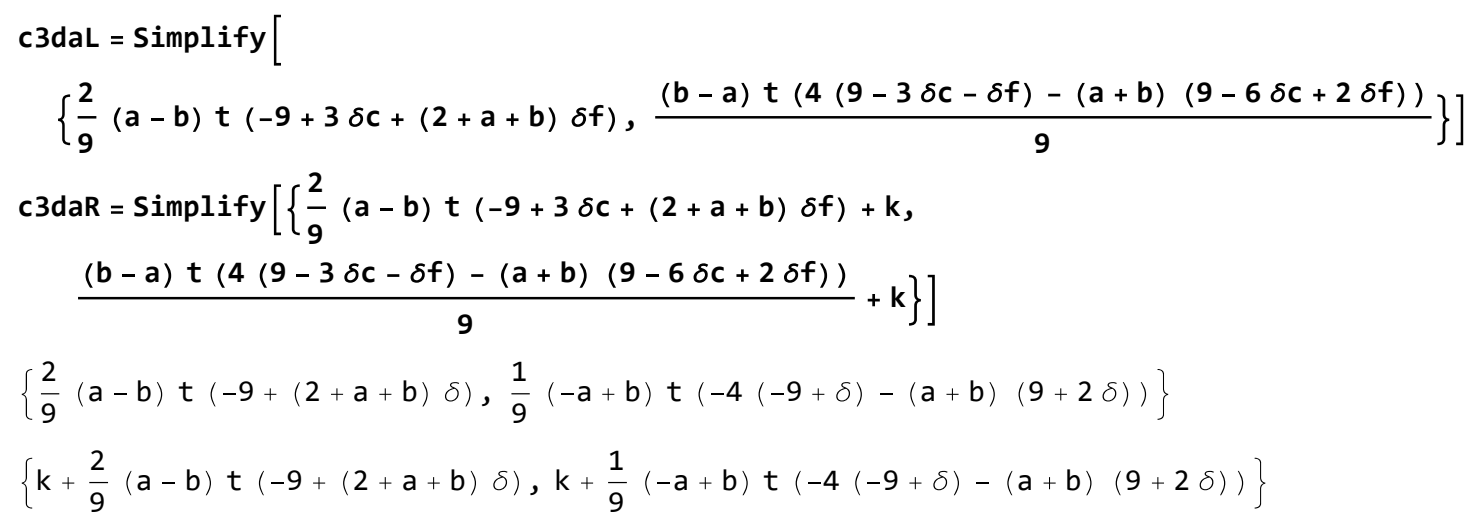

where $k$ is a sufficient large positive number (to keep $p_{A}$ at the monopoly price leading to $z=1$ ). Note that the left endpoint of Firm A's reaction function in (Case iii)' coincides with the right endpoint of Firm A's reaction function in (Case iii).

Next, from firm B's reaction function derived the proof of Proposition 1, we check the endpoints of each of the three line segments corresponding to the three cases: (i) $0 \leq \mathrm{z} \leq(\mathrm{a}+\mathrm{b}) / 4$, (ii) $(\mathrm{a}+\mathrm{b}) / 4<\mathrm{z}<(2+\mathrm{a}+\mathrm{b}) / 4$, (iii) $(2+\mathrm{a}+\mathrm{b}) / 4 \leq \mathrm{z} \leq 1$.

(Case i): The first endpoint below locates the left-hand side of Firm B's reaction function, and the second endpoint below locates the right-hand side of Firm B's reaction function in the first period.

$$
\begin{aligned}
& c 1 b L=\text { FullSimplify }\left[\left\{\frac{(b-a) t(18-3(a+b)(\delta c-\delta f)-8 \delta f)}{9},\right.\right. \\
& \left.\left.-\frac{1}{18}(a-b) t(3(-4+a+b)(-3+\delta c)+2(-8+3 a+3 b) \delta f)\right\}\right] \\
& c 1 b R=\text { Simplify }\left[\left\{\frac{(b-a) t(18+(a+b)(9-6 \delta c+2 \delta f)-8 \delta f)}{9}\right.\right. \text {, } \\
& \left.\left.-\frac{2}{9}(a-b) t(9-3 \delta c+(-4+a+b) \delta f)\right\}\right] \\
& \left\{\frac{1}{9}(-a+b) t(18-8 \delta+3(a+b) \delta),-\frac{1}{18}(a-b) t(-9(-4+a+b)+2(-8+3 a+3 b) \delta)\right\} \\
& \left\{\frac{1}{9}(-a+b) t(18-8 \delta+(a+b)(9+2 \delta)),-\frac{2}{9}(a-b) t(9+(-4+a+b) \delta)\right\}
\end{aligned}
$$

(Case i)': When $\mathrm{z}=0$, The reaction function of Firm B consists of the segment connecting the following two points.

c1dbL =

$$
\begin{aligned}
& \text { Simplify }\left[\left\{\frac{(b-a) t(18+(a+b)(9-6 \delta c+2 \delta f)-8 \delta f)}{9},-\frac{2}{9}(a-b) t(9-3 \delta c+(-4+a+b) \delta f)\right\}\right] \\
& \text { c1dbR = Simplify }\left[\left\{\frac{(b-a) t(18+(a+b)(9-6 \delta c+2 \delta f)-8 \delta f)}{9}+k\right.\right. \\
& \left.\left.\quad-\frac{2}{9}(a-b) t(9-3 \delta c+(-4+a+b) \delta f)+k\right\}\right] \\
& \left\{\frac{1}{9}(-a+b) t(18-8 \delta+(a+b)(9+2 \delta)),-\frac{2}{9}(a-b) t(9+(-4+a+b) \delta)\right\} \\
& \left\{k+\frac{1}{9}(-a+b) t(18-8 \delta+(a+b)(9+2 \delta)), k-\frac{2}{9}(a-b) t(9+(-4+a+b) \delta)\right\}
\end{aligned}
$$

where $k$ is a sufficient large positive number (to keep $p_{B}$ at the monopoly price leading to $z=0$ ). Note that the left-hand endpoint of Firm A's reaction function in case (i)' coincides with the 
right-hand endpoint of Firm A's reaction function in case (i).

Case (ii): The first endpoint below locates the left-hand side of Firm B's reaction function, and the second endpoint below locates the right-hand side of Firm B's reaction function in the first period.

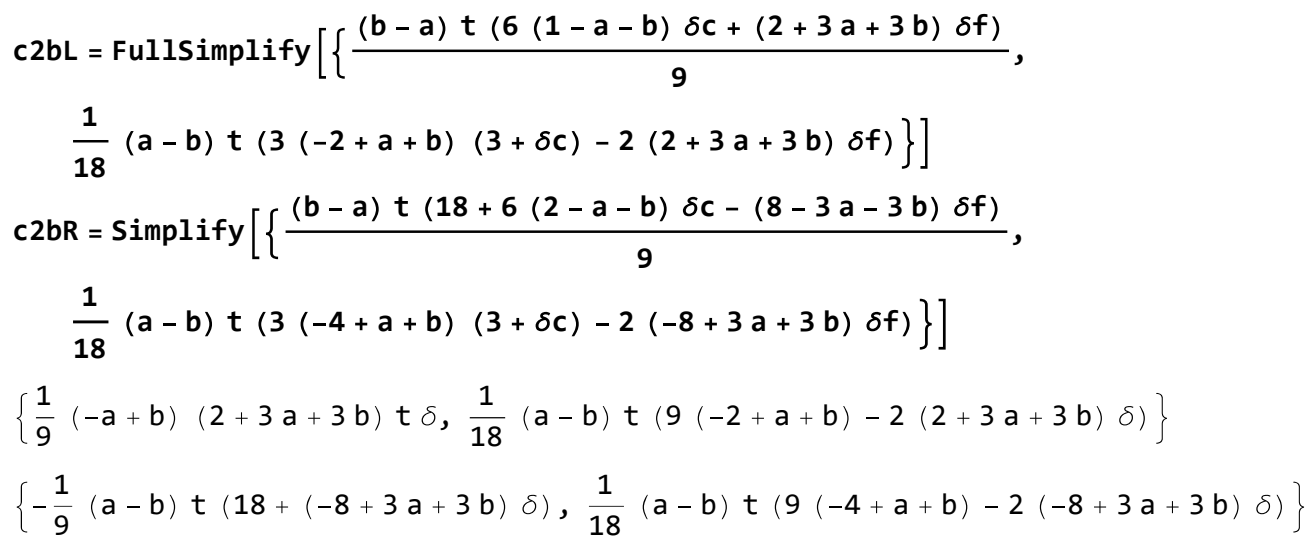

We find that the left endpoint of Firm B's reaction function in (Case i) coincides with the right endpoint of Firm B's reaction function in (Case ii). That is, Firm B's reaction function is continuous in (Case i) and (Case ii).

Case (iii): The first endpoint below locates the left-hand side of Firm B's reaction function, and the second endpoint below locates the right-hand side of Firm B's reaction function in the first period.

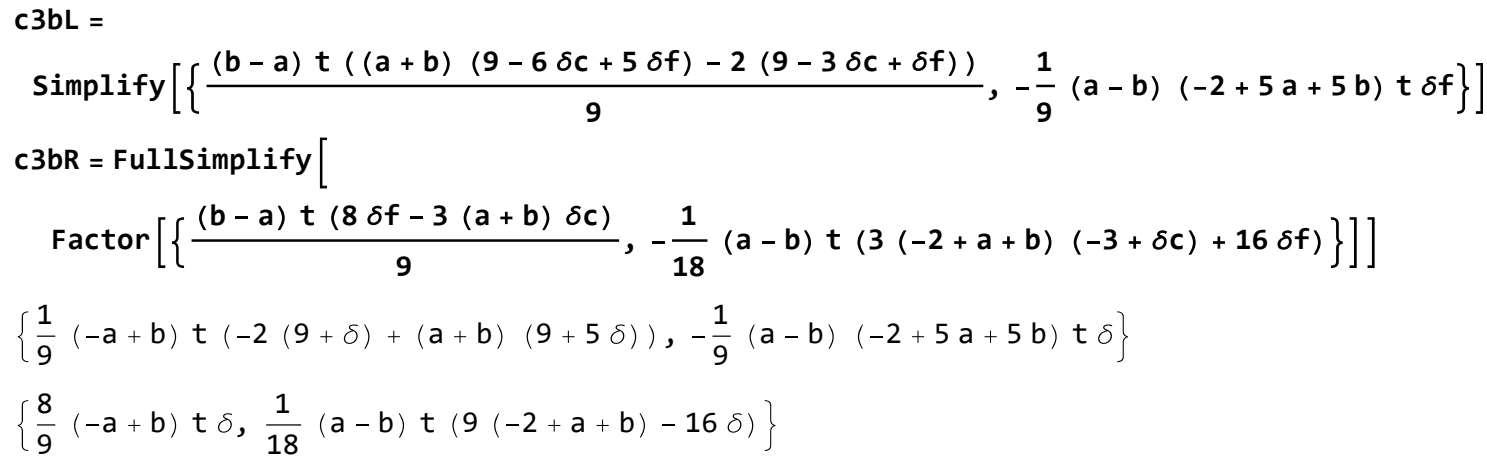

At this stage, we cannot say if Firm B's reaction function is continuous in (Case iii) and (Case ii). We will come back to this shortly. Note that the right-hand endpoint in (Case iii) corresponds to $(2+a+b) / 4=z$.

We now turn to the pricing equilibrium in the first period. To this end, we need to find the 'true' reaction function for each firm, by checking when each firm's reaction leads to a global optimum. Next we check when the two 'true' reaction functions intersect. As before, calculations are quite messy although the logical steps are identical. If necessary, readers can jump directly to the stage where we show that the pricing equilibrium exists only in (Case ii) and for all values of $\delta$.

For illustrative purposes, we start with an example where we set $\mathrm{a}=0, \mathrm{~b}=1, \mathrm{t}=1$, and $\delta=1 / 2$. 


$a=0$
$b=1$
$\delta=1 / 2$
$t=1$
$k=1$
0
1
$\frac{1}{2}$
1
1

First, we plot Firm A's reaction function corresponding to the three cases.
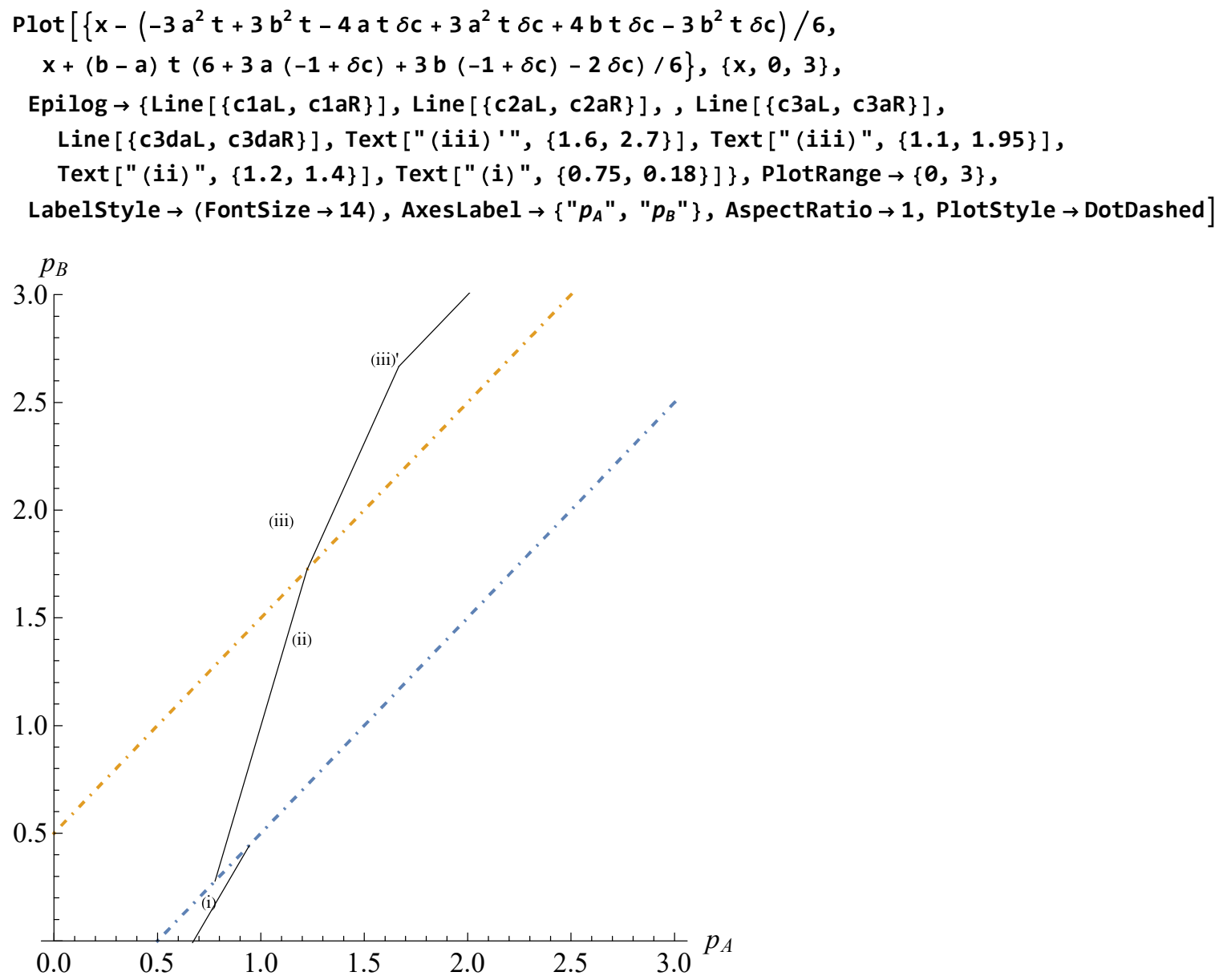

As shown above, for some $p_{B}$, there are two local optimal prices for Firm A.

We can show that the multiplicity of local optimal prices always appears.

Clear $[a, b, \delta, t, k]$

To show the multiplicity, we check the locations of the three endpoints: The left-hand endpoint in (ii) (c2aL), the left-hand and right-hand endpoints (c1aL and c1aR) in (i) (see below) 


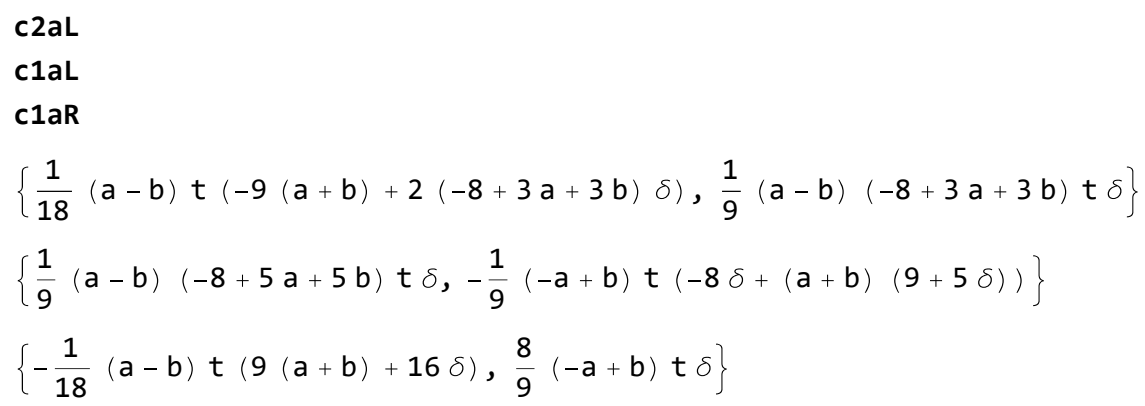

First, we compare the elements of the left-hand endpoint in (ii) and the right-hand endpoint in (i):

Factor $\left[\frac{1}{18}(a-b) t(-9(a+b)+2(-8+3 a+3 b) \delta)-\left(-\frac{1}{18}(a-b) t(9(a+b)+16 \delta)\right)\right]$

Factor $\left[\frac{1}{9}(a-b)(-8+3 a+3 b)\right.$ t $\delta-\frac{8}{9}(-a+b)$ t $\left.\delta\right]$

$\frac{1}{3}(a-b)(a+b) t \delta$

$\frac{1}{3}(a-b)(a+b) t \delta$

The outcome means that for any $\mathrm{a} \in[0,1], \mathrm{b} \in[0,1](\mathrm{a} \geq \mathrm{b}), \mathrm{t}$, and $\delta$, the left-hand endpoint in (ii) is located below the right-hand endpoint in (i) as in the above Figure.

Second, we compare the elements of the left-hand endpoint in (ii) and the left-hand endpoint in (i):

$$
\begin{aligned}
& \text { Factor }\left[\frac{1}{18}(a-b) t(-9(a+b)+2(-8+3 a+3 b) \delta)-\frac{1}{9}(a-b)(-8+5 a+5 b) t \delta\right] \\
& \text { Factor }\left[\frac{1}{9}(a-b)(-8+3 a+3 b) t \delta-\left(-\frac{1}{9}(-a+b) t(-8 \delta+(a+b)(9+5 \delta))\right)\right] \\
& -\frac{1}{18}(a-b)(a+b) t(9+4 \delta) \\
& -\frac{1}{9}(a-b)(a+b) t(9+2 \delta)
\end{aligned}
$$

The outcome means that for any $\mathrm{a} \in[0,1], \mathrm{b} \in[0,1](\mathrm{a} \geq \mathrm{b}), \mathrm{t}$, and $\delta$, the left-hand endpoint in (ii) is located above the left-hand endpoint in (i) as in the above Figure.

We need to find the global optimal price of Firm A, $p_{A}$, when there are two local optima for a given $p_{B}$. There is a price $p_{B}$ such that choosing the reaction function in (ii) and choosing the reaction function in (i) are indifferent for Firm A. This $p_{B}$ is the threshold for which choosing the reaction function in (ii) is preferred by Firm $\mathrm{A}$ if $p_{B}$ is larger than this threshold $p_{B}$, otherwise, choosing the reaction function in (i) is preferred by Firm A. We need to find the threshold value of $p_{B}$.

To check the threshold value of $p_{B}$ for Firm A's reaction function, we derive the profits under cases (ii) and (i).

The interior profit of firm $\mathrm{A}$ under case (ii) for $p_{B}$ is 


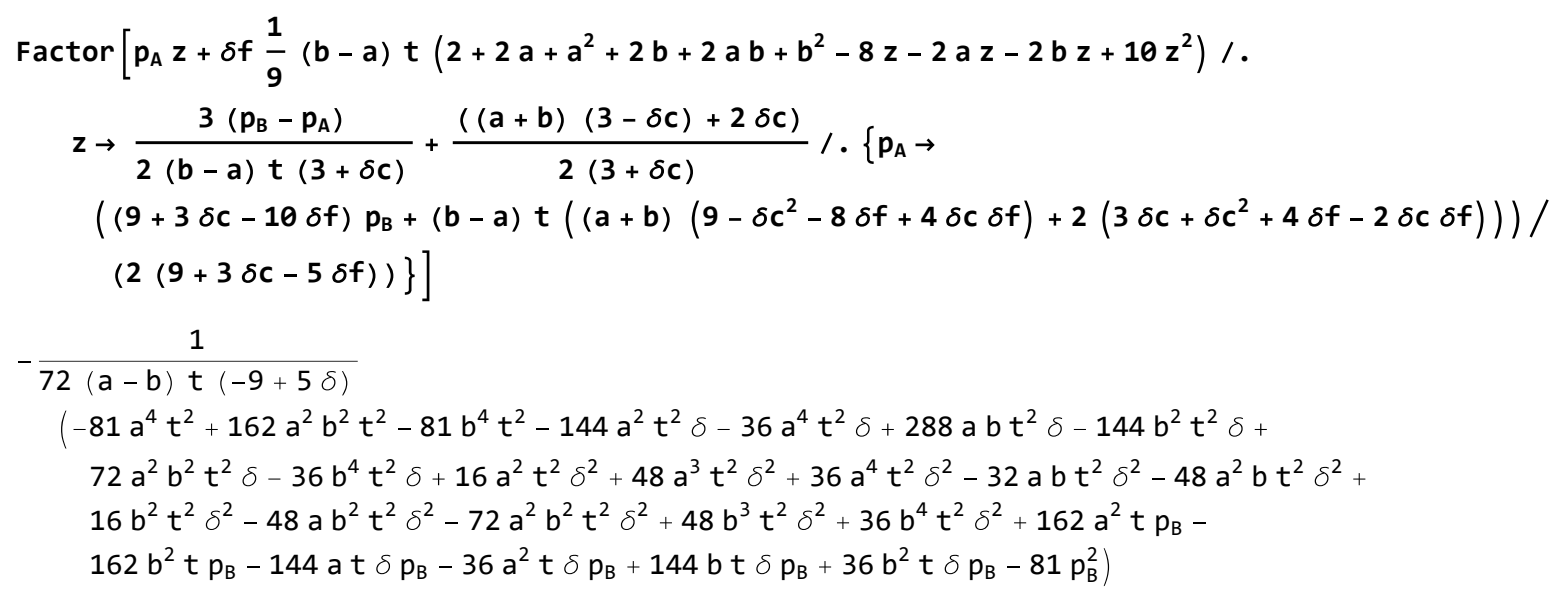

The interior profit of firm A under case (i) for $p_{B}$ is

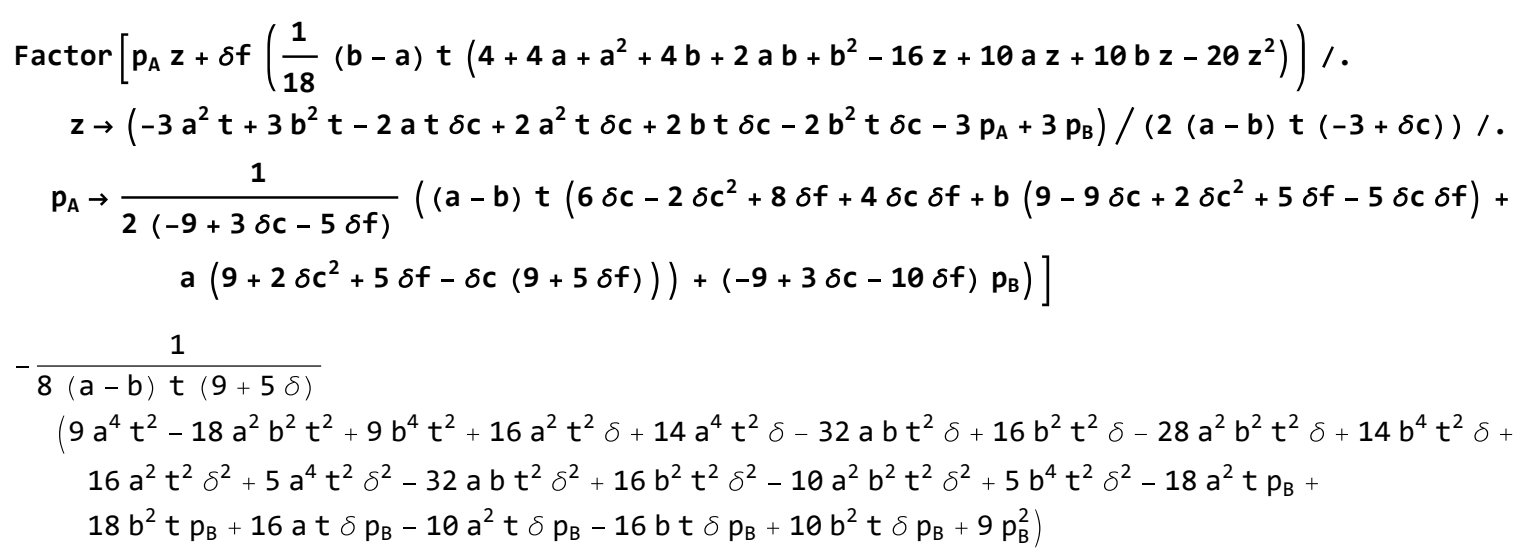

We derive the threshold value of $p_{B}$ by finding $p_{B}$ that equalizes the above two profits:

Fullsimplify [

$$
\begin{aligned}
& \text { Solve }\left[\left\{-\frac{1}{72(a-b) t(-9+5 \delta)}\left(-81 a^{4} t^{2}+162 a^{2} b^{2} t^{2}-81 b^{4} t^{2}-144 a^{2} t^{2} \delta-36 a^{4} t^{2} \delta+288 a b t^{2} \delta-\right.\right.\right. \\
& 144 b^{2} t^{2} \delta+72 a^{2} b^{2} t^{2} \delta-36 b^{4} t^{2} \delta+16 a^{2} t^{2} \delta^{2}+48 a^{3} t^{2} \delta^{2}+36 a^{4} t^{2} \delta^{2}-32 a b t^{2} \delta^{2}- \\
& 48 a^{2} b t^{2} \delta^{2}+16 b^{2} t^{2} \delta^{2}-48 a b^{2} t^{2} \delta^{2}-72 a^{2} b^{2} t^{2} \delta^{2}+48 b^{3} t^{2} \delta^{2}+36 b^{4} t^{2} \delta^{2}+ \\
& \left.162 a^{2} t p_{B}-162 b^{2} t p_{B}-144 a t \delta p_{B}-36 a^{2} t \delta p_{B}+144 b t \delta p_{B}+36 b^{2} t \delta p_{B}-81 p_{B}^{2}\right)== \\
& -\frac{1}{8(a-b) t(9+5 \delta)}\left(9 a^{4} t^{2}-18 a^{2} b^{2} t^{2}+9 b^{4} t^{2}+16 a^{2} t^{2} \delta+14 a^{4} t^{2} \delta-32 a b t^{2} \delta+16 b^{2} t^{2} \delta-\right. \\
& 28 a^{2} b^{2} t^{2} \delta+14 b^{4} t^{2} \delta+16 a^{2} t^{2} \delta^{2}+5 a^{4} t^{2} \delta^{2}-32 a b t^{2} \delta^{2}+16 b^{2} t^{2} \delta^{2}-10 a^{2} b^{2} t^{2} \delta^{2}+ \\
& \left.\left.\left.\left.5 b^{4} t^{2} \delta^{2}-18 a^{2} t p_{B}+18 b^{2} t p_{B}+16 a t \delta p_{B}-10 a^{2} t \delta p_{B}-16 b t \delta p_{B}+10 b^{2} t \delta p_{B}+9 p_{B}^{2}\right)\right\}, p_{B}\right]\right] \\
& \left\{\left\{p_{B} \rightarrow \frac{1}{90 \delta} t\left(-80 a \delta^{2}+80 b \delta^{2}+3 a^{2} \delta(9+5 \delta)-3 b^{2} \delta(9+5 \delta)+3\left(81-25 \delta^{2}\right) \sqrt{-\frac{\left(a^{2}-b^{2}\right)^{2} \delta^{2}}{-81+25 \delta^{2}}}\right)\right\},\right. \\
& \left.\left\{\mathrm{p}_{\mathrm{B}} \rightarrow \frac{1}{90 \delta} \mathrm{t}\left(-80 \mathrm{a} \delta^{2}+80 \mathrm{~b} \delta^{2}+3 \mathrm{a}^{2} \delta(9+5 \delta)-3 \mathrm{~b}^{2} \delta(9+5 \delta)+3 \sqrt{-\frac{\left(\mathrm{a}^{2}-\mathrm{b}^{2}\right)^{2} \delta^{2}}{-81+25 \delta^{2}}}\left(-81+25 \delta^{2}\right)\right)\right\}\right\}
\end{aligned}
$$

We can easily show that the latter outcome is negative. So, we use the former one.

We simplify the expression of the latter outcome, and obtain the following $p_{B}$ :

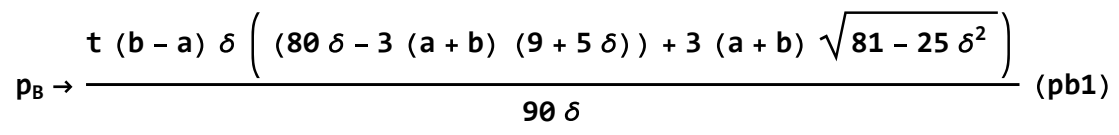


We rewrite the locations of the two endpoints: The right-hand endpoint in (i), the left-hand endpoints in (ii) (see below)

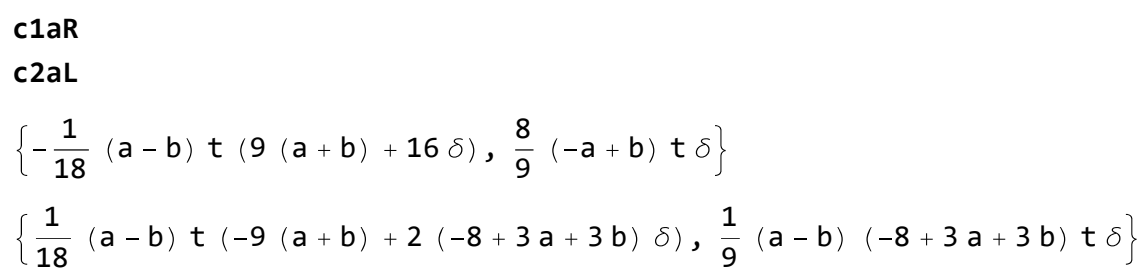

We check the condition that the derived $p_{B}(\mathrm{pb} 1)$ is below the $p_{B}$-element of the right-hand endpoints in (i).

Simplify $\left[\right.$ Factor $\left.\left[\frac{8}{9}(-a+b) t \delta-\frac{t(b-a) \delta\left((80 \delta-3(a+b)(9+5 \delta))+3(a+b) \sqrt{81-25 \delta^{2}}\right)}{90 \delta}\right]\right]$

$\frac{1}{30}(a-b)(a+b) t\left(-9-5 \delta+\sqrt{81-25 \delta^{2}}\right)$

This is positive.

We also rewrite the location of the endpoint: The left endpoints in (i) (see below) c1al

$\left\{\frac{1}{9}(a-b)(-8+5 a+5 b) t \delta,-\frac{1}{9}(-a+b) t(-8 \delta+(a+b)(9+5 \delta))\right\}$

We check the condition that the derived $p_{B}(\mathrm{pb} 1)$ is above the $p_{B}$-element of the left endpoints in (i).

Simplify $\left[\right.$ Factor $\left[\frac{t(b-a) \delta\left((80 \delta-3(a+b)(9+5 \delta))+3(a+b) \sqrt{81-25 \delta^{2}}\right)}{90 \delta}-\right.$

$$
\begin{array}{r}
\left.\left.\left(-\frac{1}{9}(-a+b) t(-8 \delta+(a+b)(9+5 \delta))\right)\right]\right] \\
-\frac{1}{90}(a-b)(a+b) t\left(63+35 \delta+3 \sqrt{81-25 \delta^{2}}\right)
\end{array}
$$

This is positive.

For the threshold value of $p_{B}(\mathrm{pb} 1)$, the point of $p_{A}$-element in Firm A's reaction function in (i) is

$$
\begin{aligned}
& \text { Simplify }[\text { Expand }[ \\
& p_{A} \rightarrow \frac{1}{2(-9+3 \delta c-5 \delta f)}\left(( a - b ) t \left(6 \delta c-2 \delta c^{2}+8 \delta f+4 \delta c \delta f+b\left(9-9 \delta c+2 \delta c^{2}+5 \delta f-5 \delta c \delta f\right)+\right.\right. \\
& \left.\left.a\left(9+2 \delta c^{2}+5 \delta f-\delta c(9+5 \delta f)\right)\right)+(-9+3 \delta c-10 \delta f) p_{B}\right) / . \\
& \left.\left.p_{B} \rightarrow \frac{1}{90 \delta} t(b-a) \delta\left((80 \delta-3(a+b)(9+5 \delta))+3(a+b) \sqrt{81-25 \delta^{2}}\right)\right]\right] \\
& \mathrm{p}_{\mathrm{A}} \rightarrow \\
& -\frac{1}{180(9+5 \delta)}(a-b) t\left(160 \delta(9+5 \delta)+3 a\left(-50 \delta^{2}+9\left(21+\sqrt{81-25 \delta^{2}}\right)+5 \delta\left(3+2 \sqrt{81-25 \delta^{2}}\right)\right)+\right. \\
& \left.3 b\left(-50 \delta^{2}+9\left(21+\sqrt{81-25 \delta^{2}}\right)+5 \delta\left(3+2 \sqrt{81-25 \delta^{2}}\right)\right)\right) \\
& p_{A} \rightarrow \frac{(b-a) t}{180(9+5 \delta)}\left(160 \delta(9+5 \delta)+3(a+b)\left((9+5 \delta)(21-10 \delta)+(9+10 \delta) \sqrt{81-25 \delta^{2}}\right)\right)
\end{aligned}
$$

The jumping point of Firm A's reaction function in (i) is defined as clja 


$$
\begin{aligned}
& \mathrm{c} 1 \mathrm{ja}=\left\{\frac{(\mathrm{b}-\mathrm{a}) \mathrm{t}}{180(9+5 \delta)}\left(160 \delta(9+5 \delta)+3(\mathrm{a}+\mathrm{b})\left((9+5 \delta)(21-10 \delta)+(9+10 \delta) \sqrt{81-25 \delta^{2}}\right)\right),\right. \\
& \left.\frac{\mathrm{t}(\mathrm{b}-\mathrm{a}) \delta\left((80 \delta-3(\mathrm{a}+\mathrm{b})(9+5 \delta))+3(\mathrm{a}+\mathrm{b}) \sqrt{81-25 \delta^{2}}\right)}{90 \delta}\right\} \\
& \left\{\frac{1}{180(9+5 \delta)}(-\mathrm{a}+\mathrm{b}) \mathrm{t}\left(160 \delta(9+5 \delta)+3(\mathrm{a}+\mathrm{b})\left((21-10 \delta)(9+5 \delta)+(9+10 \delta) \sqrt{81-25 \delta^{2}}\right)\right),\right. \\
& \left.\frac{1}{90}(-\mathrm{a}+\mathrm{b}) \mathrm{t}\left(80 \delta-3(\mathrm{a}+\mathrm{b})(9+5 \delta)+3(\mathrm{a}+\mathrm{b}) \sqrt{81-25 \delta^{2}}\right)\right\}
\end{aligned}
$$

Also, for the threshold value of $p_{B}(\mathrm{pb} 1)$, the point of $p_{A}$-element in Firm A's reaction function in (ii) is

$$
\begin{aligned}
& \text { Simplify }\left[\text { Expand } \left[p_{A} \rightarrow\right.\right. \\
& \left((9+3 \delta c-10 \delta f) p_{B}+(b-a) t\left((a+b)\left(9-\delta c^{2}-8 \delta f+4 \delta c \delta f\right)+2\left(3 \delta c+\delta c^{2}+4 \delta f-2 \delta c \delta f\right)\right)\right) / \\
& \left.\left.(2(9+3 \delta c-5 \delta f)) / . p_{B} \rightarrow \frac{1}{90 \delta} t(b-a) \delta\left((80 \delta-3(a+b)(9+5 \delta))+3(a+b) \sqrt{81-25 \delta^{2}}\right)\right]\right] \\
& p_{A} \rightarrow \\
& \begin{array}{l}
\frac{1}{180(-9+5 \delta)}(\mathrm{a}-\mathrm{b}) \mathrm{t}\left(160(9-5 \delta) \delta+3 \mathrm{a}\left(50 \delta^{2}+9\left(21+\sqrt{81-25 \delta^{2}}\right)-5 \delta\left(39+2 \sqrt{81-25 \delta^{2}}\right)\right)+\right. \\
\left.\quad 3 \mathrm{~b}\left(50 \delta^{2}+9\left(21+\sqrt{81-25 \delta^{2}}\right)-5 \delta\left(39+2 \sqrt{81-25 \delta^{2}}\right)\right)\right) \\
\mathrm{p}_{\mathrm{A}} \rightarrow \frac{(\mathrm{b}-\mathrm{a}) \mathrm{t}}{180(9-5 \delta)}\left(160(9-5 \delta) \delta+3(\mathrm{a}+\mathrm{b})\left((9-5 \delta)(21-10 \delta)+(9-10 \delta) \sqrt{81-25 \delta^{2}}\right)\right)
\end{array}
\end{aligned}
$$

The jumping point of Firm A's reaction function in (ii) is defined as c2ja

$$
\begin{aligned}
& c 2 j a=\left\{\frac{(b-a) t}{180(9-5 \delta)}\left(160(9-5 \delta) \delta+3(a+b)\left((9-5 \delta)(21-10 \delta)+(9-10 \delta) \sqrt{81-25 \delta^{2}}\right)\right),\right. \\
& \left.\frac{t(b-a) \delta\left((80 \delta-3(a+b)(9+5 \delta))+3(a+b) \sqrt{81-25 \delta^{2}}\right)}{90 \delta}\right\} \\
& \left\{\frac{1}{180(9-5 \delta)}(-a+b) t\left(160(9-5 \delta) \delta+3(a+b)\left((21-10 \delta)(9-5 \delta)+(9-10 \delta) \sqrt{81-25 \delta^{2}}\right)\right),\right. \\
& \left.\frac{1}{90}(-a+b) t\left(80 \delta-3(a+b)(9+5 \delta)+3(a+b) \sqrt{81-25 \delta^{2}}\right)\right\}
\end{aligned}
$$

Next we show various examples of Firm A's 'true' reaction function for different values of $a, b$, $t, \delta$, and $k$.

$$
\begin{aligned}
& a=0 \\
& b=1 \\
& \delta=1 / 2 \\
& t=1 \\
& k=2 \\
& 0 \\
& 1 \\
& \frac{1}{2} \\
& 1 \\
& 2
\end{aligned}
$$




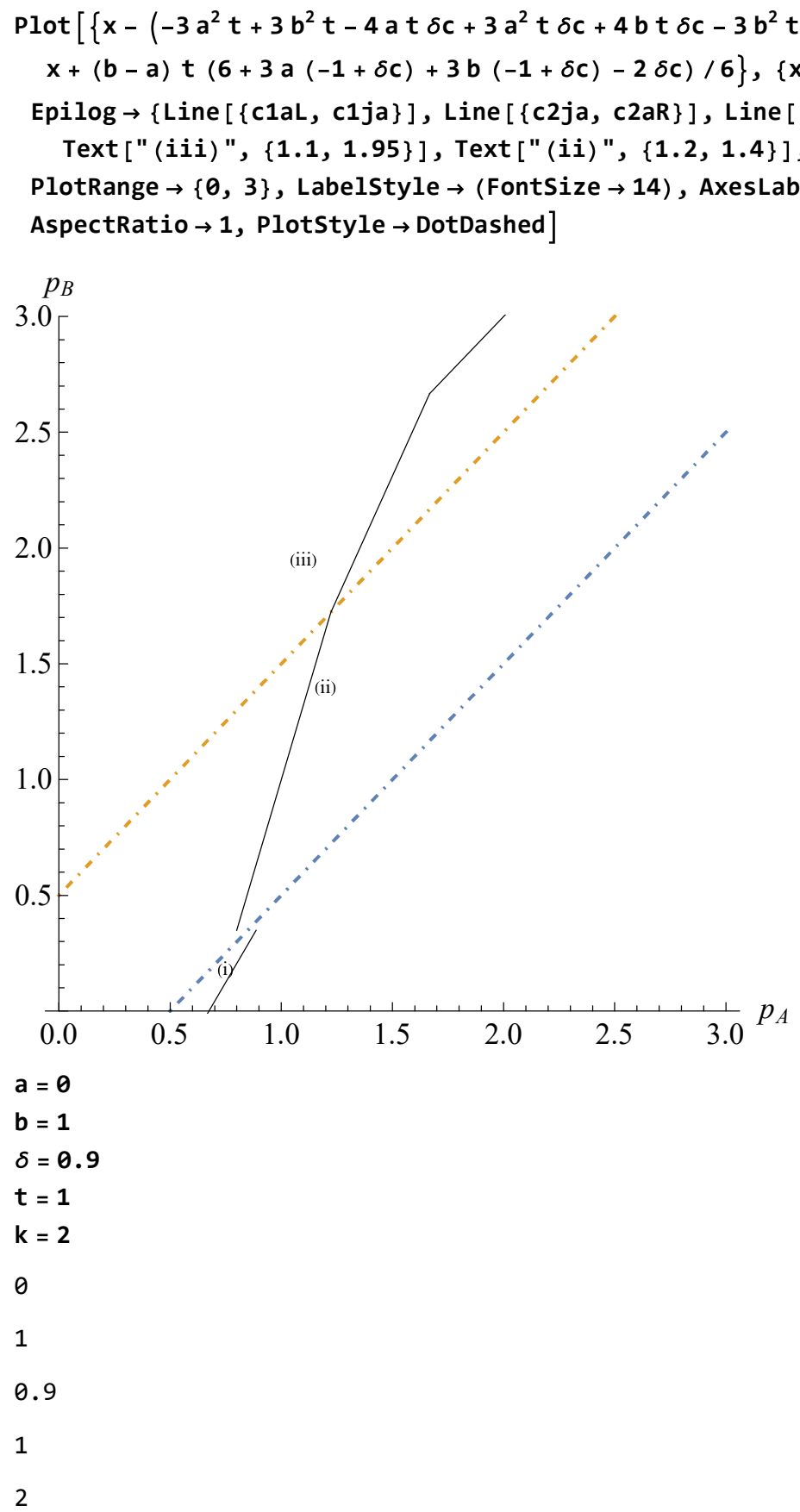




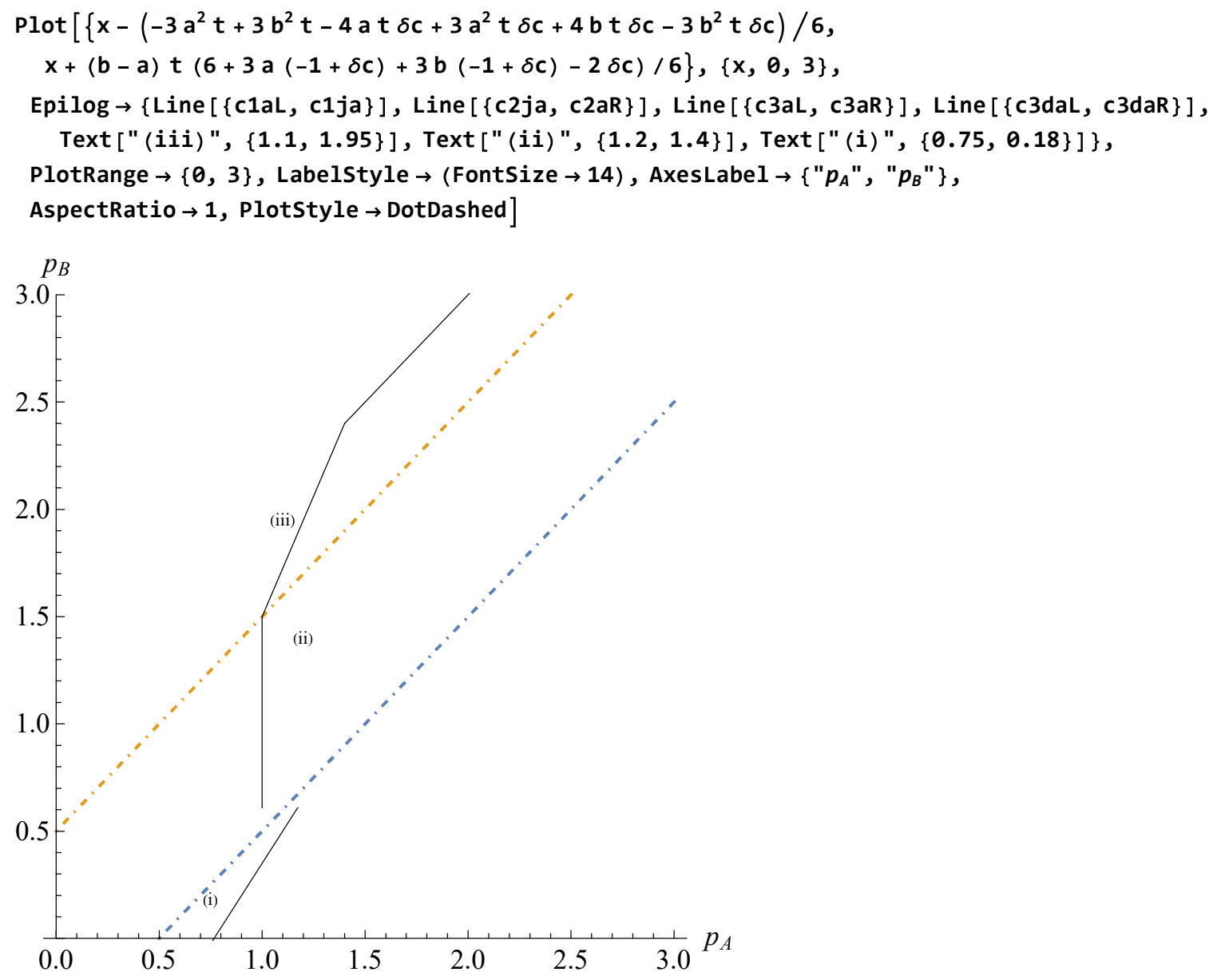

Clear $[a, b, \delta, t, k]$

We now turn to Firm B's reaction function in the three cases. As before, we start with an example by setting $a=0, b=1, t=1$, and $\delta=1 / 2$ :

$a=0$
$b=1$
$\delta=4 / 5$
$t=1$
$k=1$
0
1
$\frac{4}{5}$
1
1




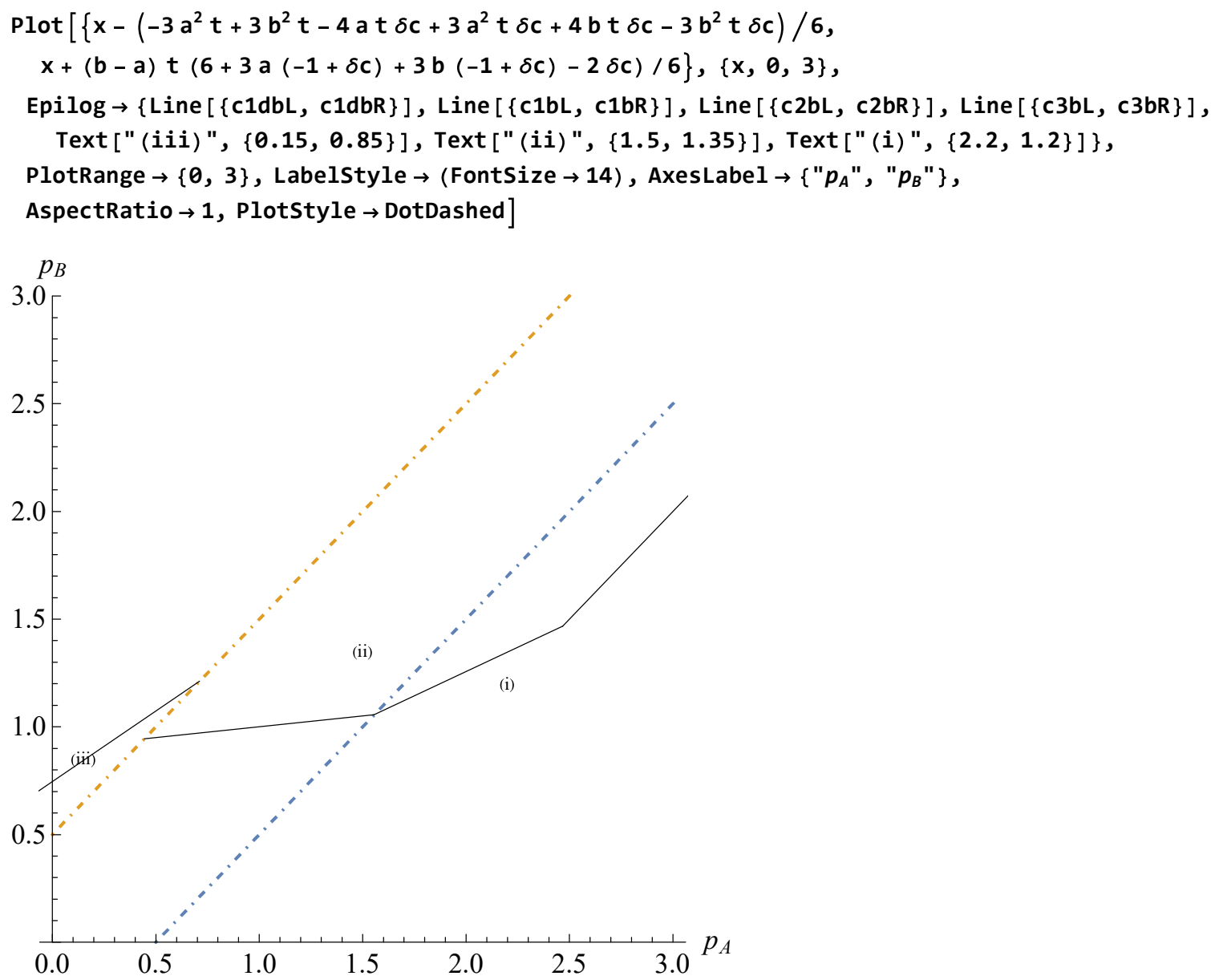

We find that for some $p_{A}$, there are two local optimal prices for Firm B. We can show that the multiplicity of local optimal prices always appears.

clear $[a, b, \delta, t, k]$

To show the multiplicity, we check the locations of the two endpoints: The left-hand endpoint in (ii) (c2bL) and the left-hand and right-hand endpoints (c3bL and c3bR) in (iii) (see below)

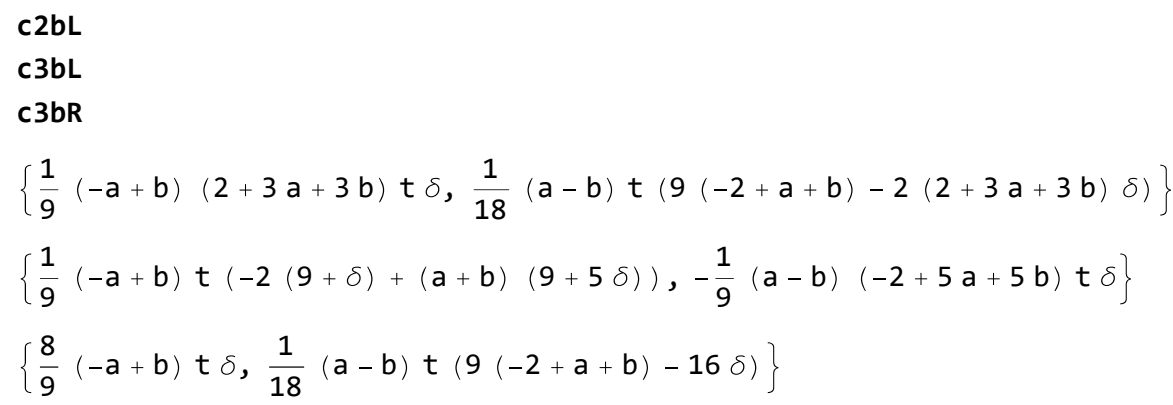

First, we compare the elements of the left-hand endpoint in (ii) and the right-hand endpoint in (iii): 


$$
\begin{aligned}
& \text { Factor }\left[\frac{1}{9}(-a+b)(2+3 a+3 b) t \delta-\frac{8}{9}(-a+b) t \delta\right] \\
& \text { Factor }\left[\frac{1}{18}(a-b) t(9(-2+a+b)-2(2+3 a+3 b) \delta)-\left(\frac{1}{18}(a-b) t(9(-2+a+b)-16 \delta)\right)\right] \\
& -\frac{1}{3}(a-b)(-2+a+b) \text { t } \delta \\
& -\frac{1}{3}(a-b)(-2+a+b) \text { t } \delta
\end{aligned}
$$

The outcome means that for any $\mathrm{a} \in[0,1], \mathrm{b} \in[0,1](\mathrm{a} \geq \mathrm{b}), \mathrm{t}$, and $\delta$, the right-hand endpoint in (iii) is located above the left-hand endpoint in (ii) as in the above Figure.

Second, we compare the elements of the left-hand endpoint in (ii) and the left-hand endpoint in (iii):

$$
\begin{aligned}
& \text { Factor }\left[\frac{1}{9}(-a+b)(2+3 a+3 b) t \delta-\frac{1}{9}(-a+b) t(-2(9+\delta)+(a+b)(9+5 \delta))\right] \\
& \text { Factor }\left[\frac{1}{18}(a-b) t(9(-2+a+b)-2(2+3 a+3 b) \delta)-\left(-\frac{1}{9}(a-b)(-2+5 a+5 b) t \delta\right)\right] \\
& \frac{1}{9}(a-b)(-2+a+b) t(9+2 \delta) \\
& \frac{1}{18}(a-b)(-2+a+b) t(9+4 \delta)
\end{aligned}
$$

The outcome means that for any $\mathrm{a} \in[0,1], \mathrm{b} \in[0,1](\mathrm{a} \geq \mathrm{b}), \mathrm{t}$, and $\delta$, the left-hand endpoint in (ii) is located above the left-hand endpoint in (iii) as in the above Figure.

We need to find the global optimal price of Firm B, $p_{B}$, when there are two local optima for a given $p_{A}$. There is a price $p_{A}$ such that choosing the reaction function in (ii) and choosing the reaction function in (iii) are indifferent for Firm B. This $p_{A}$ is the threshold in which choosing the reaction function in (ii) is preferred by Firm $\mathrm{B}$ if $p_{A}$ is larger than the threshold $p_{A}$, otherwise, choosing the reaction function in (iii) is preferred by Firm B. We need to find the threshold value of $p_{A}$.

To check the threshold value of $p_{A}$ for Firm B's reaction function, we derive the profits under cases (ii) and (iii).

The interior profit of firm $\mathrm{B}$ under case (ii) for $p_{A}$ is

$$
\begin{aligned}
& \text { Factor }\left[p_{B}(1-z)+\delta f \frac{1}{9}(b-a) t\left(8-4 a+a^{2}-4 b+2 a b+b^{2}-8 z-2 a z-2 b z+10 z^{2}\right) /\right. \\
& \quad z \rightarrow \frac{3\left(p_{B}-p_{A}\right)}{2(b-a) t(3+\delta c)}+\frac{((a+b)(3-\delta c)+2 \delta c)}{2(3+\delta c)} / \\
& \left\{\begin{array}{l}
p_{B} \rightarrow \frac{1}{2(9+3 \delta c-5 \delta f)}(-(a-b) t(18+6 \delta c-8 \delta f+4 \delta c \delta f+ \\
\left.\left.\left.\left.a\left(-9+\delta c^{2}+8 \delta f-4 \delta c \delta f\right)+b\left(-9+\delta c^{2}+8 \delta f-4 \delta c \delta f\right)\right)+(9+3 \delta c-10 \delta f) p_{A}\right)\right\}\right]
\end{array}\right. \\
& \begin{array}{l}
72(a-b) t(-9+5 \delta) \\
\left(-324 a^{2} t^{2}+324 a^{3} t^{2}-81 a^{4} t^{2}+648 a b t^{2}-324 a^{2} b t^{2}-324 b^{2} t^{2}-324 a b^{2} t^{2}+162 a^{2} b^{2} t^{2}+324 b^{3} t^{2}-\right. \\
81 b^{4} t^{2}-288 a^{2} t^{2} \delta+144 a^{3} t^{2} \delta-36 a^{4} t^{2} \delta+576 a b t^{2} \delta-144 a^{2} b t^{2} \delta-288 b^{2} t^{2} \delta-144 a b^{2} t^{2} \delta+ \\
72 a^{2} b^{2} t^{2} \delta+144 b^{3} t^{2} \delta-36 b^{4} t^{2} \delta+256 a^{2} t^{2} \delta^{2}-192 a^{3} t^{2} \delta^{2}+36 a^{4} t^{2} \delta^{2}-512 a b t^{2} \delta^{2}+ \\
192 a^{2} b t^{2} \delta^{2}+256 b^{2} t^{2} \delta^{2}+192 a b^{2} t^{2} \delta^{2}-72 a^{2} b^{2} t^{2} \delta^{2}-192 b^{3} t^{2} \delta^{2}+36 b^{4} t^{2} \delta^{2}+324 a t p_{A}- \\
\left.162 a^{2} t p_{A}-324 b t p_{A}+162 b^{2} t p_{A}-216 a t \delta p_{A}+36 a^{2} t \delta p_{A}+216 b t \delta p_{A}-36 b^{2} t \delta p_{A}-81 p_{A}^{2}\right)
\end{array}
\end{aligned}
$$

The interior profit of firm $\mathrm{B}$ under case (iii) for $p_{B}$ 


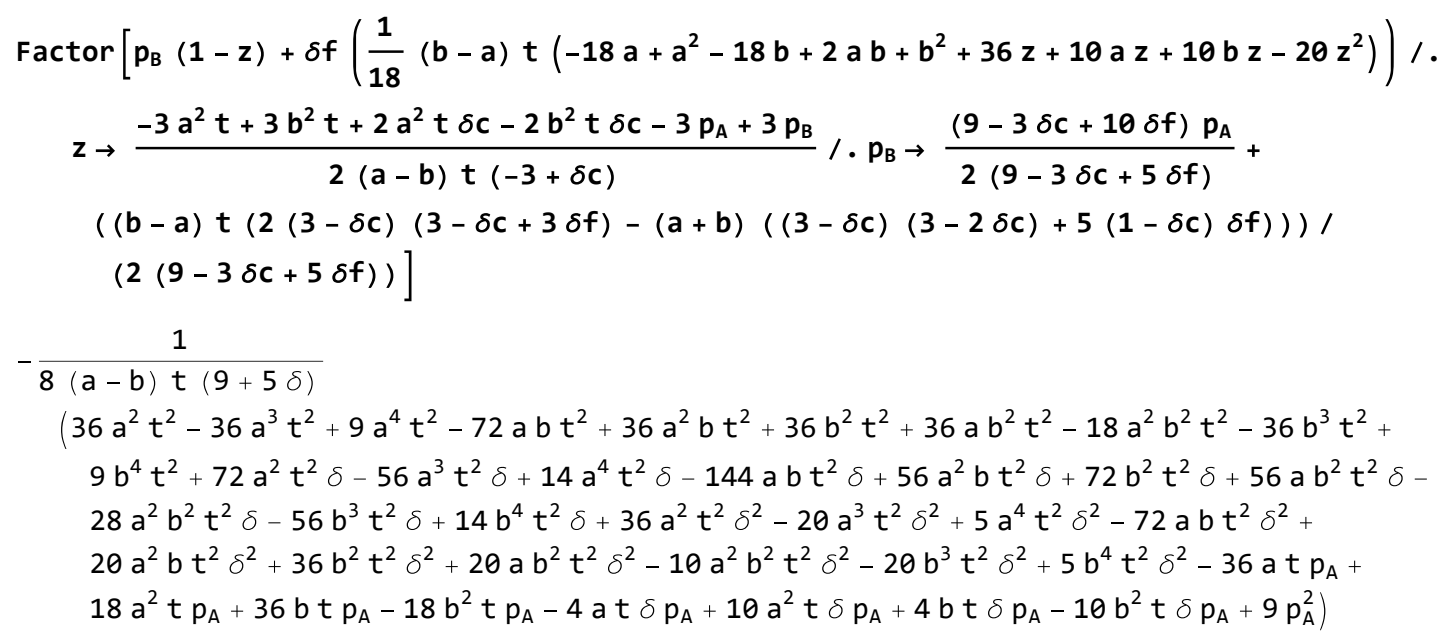

We derive the threshold value of $p_{B}$ by finding $p_{B}$ that equalizes the above two profits:

Fullsimplify [

$$
\begin{aligned}
& \text { Solve }\left[\left\{-\frac{1}{72(a-b) t(-9+5 \delta)}\left(-324 a^{2} t^{2}+324 a^{3} t^{2}-81 a^{4} t^{2}+648 a b t^{2}-324 a^{2} b t^{2}-324 b^{2} t^{2}-\right.\right.\right. \\
& 324 a b^{2} t^{2}+162 a^{2} b^{2} t^{2}+324 b^{3} t^{2}-81 b^{4} t^{2}-288 a^{2} t^{2} \delta+144 a^{3} t^{2} \delta-36 a^{4} t^{2} \delta+576 a b t^{2} \delta- \\
& 144 a^{2} b t^{2} \delta-288 b^{2} t^{2} \delta-144 a b^{2} t^{2} \delta+72 a^{2} b^{2} t^{2} \delta+144 b^{3} t^{2} \delta-36 b^{4} t^{2} \delta+256 a^{2} t^{2} \delta^{2}- \\
& 192 a^{3} t^{2} \delta^{2}+36 a^{4} t^{2} \delta^{2}-512 a b t^{2} \delta^{2}+192 a^{2} b t^{2} \delta^{2}+256 b^{2} t^{2} \delta^{2}+192 a b^{2} t^{2} \delta^{2}- \\
& 72 a^{2} b^{2} t^{2} \delta^{2}-192 b^{3} t^{2} \delta^{2}+36 b^{4} t^{2} \delta^{2}+324 a t p_{A}-162 a^{2} t p_{A}-324 b t p_{A}+162 b^{2} t p_{A}- \\
& \left.216 a t \delta p_{A}+36 a^{2} t \delta p_{A}+216 b t \delta p_{A}-36 b^{2} t \delta p_{A}-81 p_{A}^{2}\right)=-\frac{1}{8(a-b) t(9+5 \delta)} \\
& \left(36 a^{2} t^{2}-36 a^{3} t^{2}+9 a^{4} t^{2}-72 a b t^{2}+36 a^{2} b t^{2}+36 b^{2} t^{2}+36 a b^{2} t^{2}-18 a^{2} b^{2} t^{2}-36 b^{3} t^{2}+\right. \\
& 9 b^{4} t^{2}+72 a^{2} t^{2} \delta-56 a^{3} t^{2} \delta+14 a^{4} t^{2} \delta-144 a b t^{2} \delta+56 a^{2} b t^{2} \delta+72 b^{2} t^{2} \delta+56 a b^{2} t^{2} \delta- \\
& 28 a^{2} b^{2} t^{2} \delta-56 b^{3} t^{2} \delta+14 b^{4} t^{2} \delta+36 a^{2} t^{2} \delta^{2}-20 a^{3} t^{2} \delta^{2}+5 a^{4} t^{2} \delta^{2}-72 a b t^{2} \delta^{2}+ \\
& 20 a^{2} b t^{2} \delta^{2}+36 b^{2} t^{2} \delta^{2}+20 a b^{2} t^{2} \delta^{2}-10 a^{2} b^{2} t^{2} \delta^{2}-20 b^{3} t^{2} \delta^{2}+5 b^{4} t^{2} \delta^{2}-36 a t p_{A}+ \\
& \left.\left.\left.\left.18 a^{2} t p_{A}+36 b t p_{A}-18 b^{2} t p_{A}-4 a t \delta p_{A}+10 a^{2} t \delta p_{A}+4 b t \delta p_{A}-10 b^{2} t \delta p_{A}+9 p_{A}^{2}\right)\right\}, p_{A}\right]\right] \\
& \left\{\left\{p _ { A } \rightarrow \frac { 1 } { 9 0 \delta } t \left(2 a(27-25 \delta) \delta-3 a^{2} \delta(9+5 \delta)+\right.\right.\right. \\
& \left.\left.3 b^{2} \delta(9+5 \delta)+2 b \delta(-27+25 \delta)+3\left(81-25 \delta^{2}\right) \sqrt{-\frac{(a-b)^{2}(-2+a+b)^{2} \delta^{2}}{-81+25 \delta^{2}}}\right)\right\}, \\
& \left\{p _ { A } \rightarrow \frac { 1 } { 9 0 \delta } t \left(2 a(27-25 \delta) \delta-3 a^{2} \delta(9+5 \delta)+3 b^{2} \delta(9+5 \delta)+2 b \delta(-27+25 \delta)+\right.\right. \\
& \left.\left.\left.3 \sqrt{-\frac{(a-b)^{2}(-2+a+b)^{2} \delta^{2}}{-81+25 \delta^{2}}}\left(-81+25 \delta^{2}\right)\right)\right\}\right\} \\
& \mathrm{p}_{\mathrm{A}} \rightarrow \frac{1}{90 \delta} \mathrm{t}(\mathrm{b}-\mathrm{a}) \delta\left((-54+50 \delta+3(\mathrm{a}+\mathrm{b})(9+5 \delta))+3(2-(\mathrm{a}+\mathrm{b})) \sqrt{\left(81-25 \delta^{2}\right)}\right)
\end{aligned}
$$

We can easily show that the latter outcome is negative. So, we use the former one.

We simplify the expression of the latter outcome, and obtain the following $p_{B}$ :

$\mathrm{p}_{\mathrm{A}} \rightarrow \frac{1}{90 \delta} \mathrm{t}(\mathrm{b}-\mathrm{a}) \delta\left((-54+50 \delta+3(\mathrm{a}+\mathrm{b})(9+5 \delta))+3(2-(\mathrm{a}+\mathrm{b})) \sqrt{\left(81-25 \delta^{2}\right)}\right)(\mathrm{pa} 1)$

We rewrite the locations of the two endpoints: The right endpoint in (iii) and the left endpoint in (ii) (see below) 


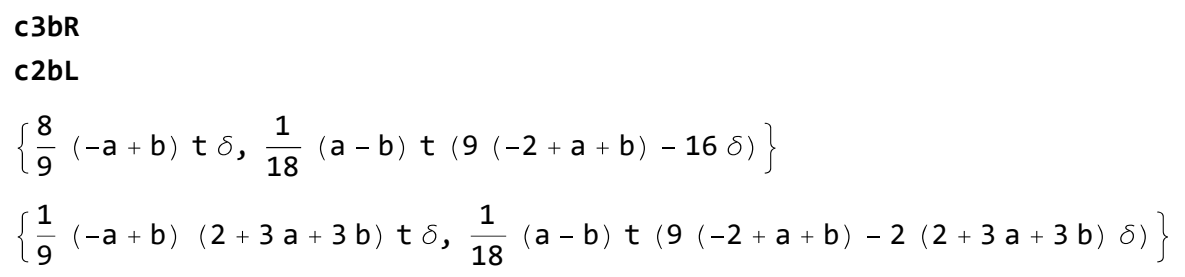

We show that the derived $p_{A}$ (pa1) is smaller than the $p_{A}$-element of the right-hand endpoints in (iii).

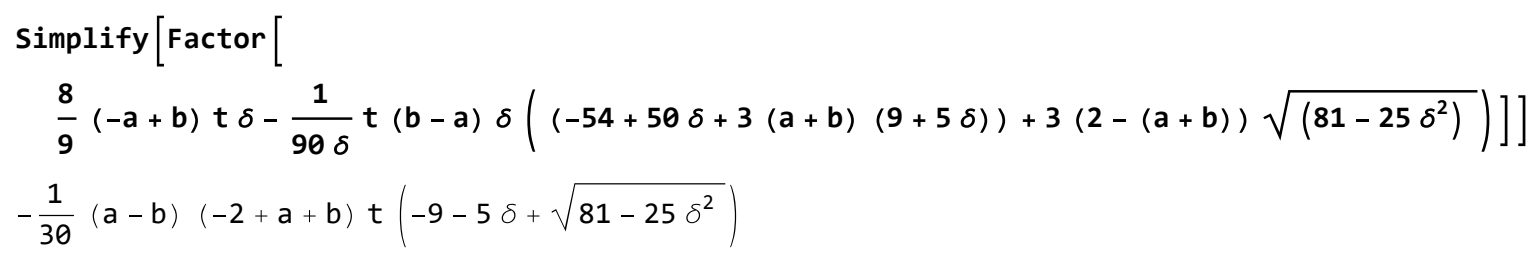

This is positive (note that $-9-5 \delta+\sqrt{81-25 \delta^{2}}$ is negative).

We show that the derived $p_{A}$ (pa1) is larger than the $p_{A}$-element of the left-hand endpoints in (ii).

Simplify $\left[\right.$ Factor $\left[\frac{1}{90 \delta} \mathrm{t}(\mathrm{b}-\mathrm{a}) \delta\left((-54+50 \delta+3(\mathrm{a}+\mathrm{b})(9+5 \delta))+3(2-(\mathrm{a}+\mathrm{b})) \sqrt{\left(81-25 \delta^{2}\right)}\right)-\right.$

$$
\begin{aligned}
& \left.\left.\frac{1}{9}(-a+b)(2+3 a+3 b) t \delta\right]\right] \\
& \frac{1}{30}(a-b)(-2+a+b) t\left(-9+5 \delta+\sqrt{81-25 \delta^{2}}\right)
\end{aligned}
$$

This is positive (note that $-9+5 \delta+\sqrt{81-25 \delta^{2}}$ is positive).

The reaction function of Firm B in (ii) is

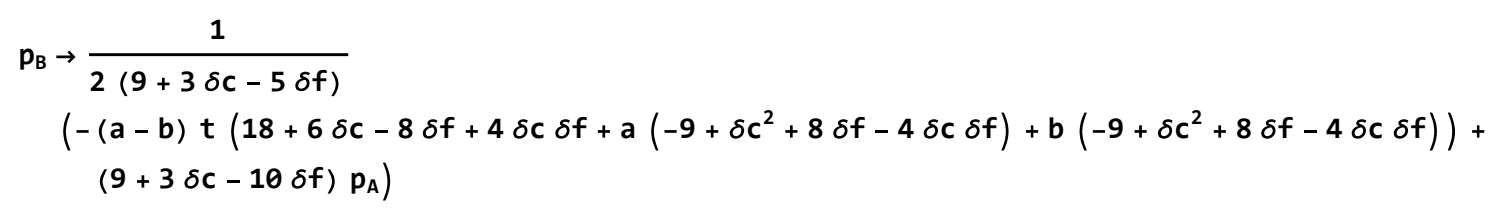

We substitute $p_{A}$ into $p_{B}$ :

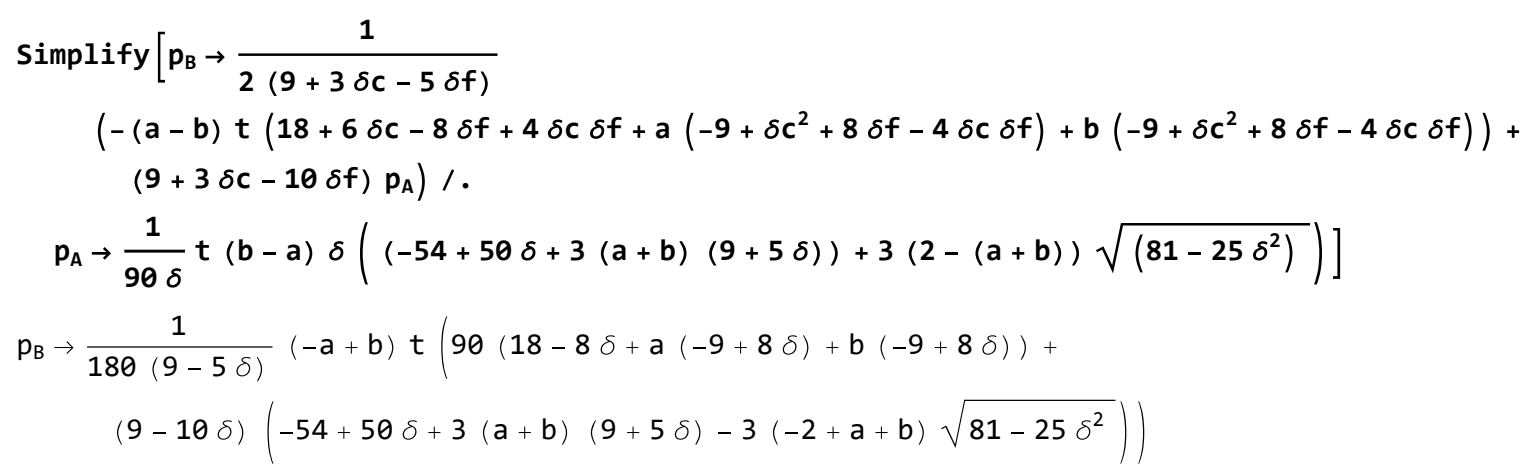




$$
\begin{aligned}
& c 2 j b=\left\{\frac{1}{90 \delta} t(b-a) \delta\left((-54+50 \delta+3(a+b)(9+5 \delta))+3(2-(a+b)) \sqrt{\left(81-25 \delta^{2}\right)}\right),\right. \\
& \frac{1}{180(9-5 \delta)}(-a+b) t(90(18-8 \delta+a(-9+8 \delta)+b(-9+8 \delta))+ \\
& \left.\left.\quad(9-10 \delta)\left(-54+50 \delta+3(a+b)(9+5 \delta)-3(-2+a+b) \sqrt{81-25 \delta^{2}}\right)\right)\right\} \\
& \left\{\frac{1}{90}(-a+b) t\left(-54+50 \delta+3(a+b)(9+5 \delta)+3(2-a-b) \sqrt{81-25 \delta^{2}}\right),\right. \\
& \frac{1}{180(9-5 \delta)}(-a+b) t(90(18-8 \delta+a(-9+8 \delta)+b(-9+8 \delta))+ \\
& \left.\left.\quad(9-10 \delta)\left(-54+50 \delta+3(a+b)(9+5 \delta)-3(-2+a+b) \sqrt{81-25 \delta^{2}}\right)\right)\right\}
\end{aligned}
$$

The reaction function of Firm B in (iii) is

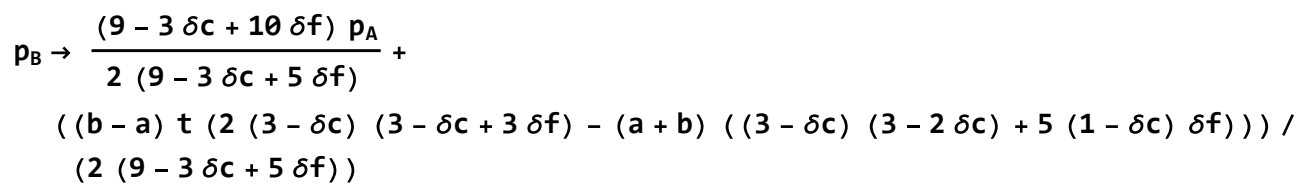

We substitute $p_{A}$ into $p_{B}$ :

$$
\begin{aligned}
& \text { Simplify }\left[p_{B} \rightarrow\right. \frac{(9-3 \delta c+10 \delta f) p_{A}}{2(9-3 \delta c+5 \delta f)}+ \\
&((b-a) t(2(3-\delta c)(3-\delta c+3 \delta f)-(a+b)((3-\delta c)(3-2 \delta c)+5(1-\delta c) \delta f))) / \\
&(2(9-3 \delta c+5 \delta f)) / . \\
& p_{A} \rightarrow\left.\frac{1}{90 \delta} t(b-a) \delta\left((-54+50 \delta+3(a+b)(9+5 \delta))+3(2-(a+b)) \sqrt{\left(81-25 \delta^{2}\right)}\right)\right] \\
& p_{B} \rightarrow \frac{1}{180(9+5 \delta)}(-a+b) t(90(18(1+\delta)-(a+b)(9+5 \delta))+ \\
& c 3 j b= \frac{1}{90 \delta} t(b-a) \delta\left((-54+50 \delta+3(a+b)(9+5 \delta))+3(2-(a+b)) \sqrt{\left(81-25 \delta^{2}\right)}\right), \\
& \frac{1}{180(9+5 \delta)}(-a+b) t(90(18(1+\delta)-(a+b)(9+5 \delta))+ \\
&\left.\left.\quad(9+10 \delta)\left(-54+50 \delta+3(a+b)(9+5 \delta)-3(-2+a+b) \sqrt{81-25 \delta^{2}}\right)\right)\right\} \\
&\left\{\frac{1}{90}(-a+b) t\left(-54+50 \delta+3(a+b)(9+5 \delta)+3(2-a-b) \sqrt{81-25 \delta^{2}}\right),\right. \\
& \frac{1}{180(9+5 \delta)}(-a+b) t(90(18(1+\delta)-(a+b)(9+5 \delta))+ \\
&\left.\left.(9+10 \delta)\left(-54+50 \delta+3(a+b)(9+5 \delta)-3(-2+a+b) \sqrt{81-25 \delta^{2}}\right)\right)\right\}
\end{aligned}
$$


c1dbL

$\left\{\frac{1}{9}(a-b)(2+a+b) t(-9+4 \delta),-\frac{2}{9}(a-b) t(9+(-7+a+b) \delta)\right\}$

Plot $\left[\left\{x-\left(-3 a^{2} t+3 b^{2} t-4 a t \delta c+3 a^{2} t \delta c+4 b t \delta c-3 b^{2} t \delta c\right) / 6\right.\right.$,

$x+(b-a) t(6+3 a(-1+\delta c)+3 b(-1+\delta c)-2 \delta c) / 6\},\{x, 0,3\}$,

Epilog $\rightarrow\{$ Line $[\{c 1 d b L, c 1 d b R\}]$, Line $[\{c 1 b R, c 1 b L\}]$, Line $[\{c 2 b R, c 2 j b\}]$,

Line $[\{c 3 j b, c 3 b L\}], \operatorname{Text}["(\mathrm{iii}) ",\{0.15,0.85\}]$, Text ["(ii)", $\{1.5,1.35\}]$,

Text ["(i) ", \{2.2, 1.2\}], Text ["(i) '", \{2.7, 1.6 $]\}$, PlotRange $\rightarrow\{0,3\}$,

LabelStyle $\rightarrow$ (FontSize $\rightarrow 14$ ), AxesLabel $\rightarrow\left\{" p_{A} ", " p_{B} "\right\}$, AspectRatio $\rightarrow 1$, PlotStyle $\rightarrow$ DotDashed]

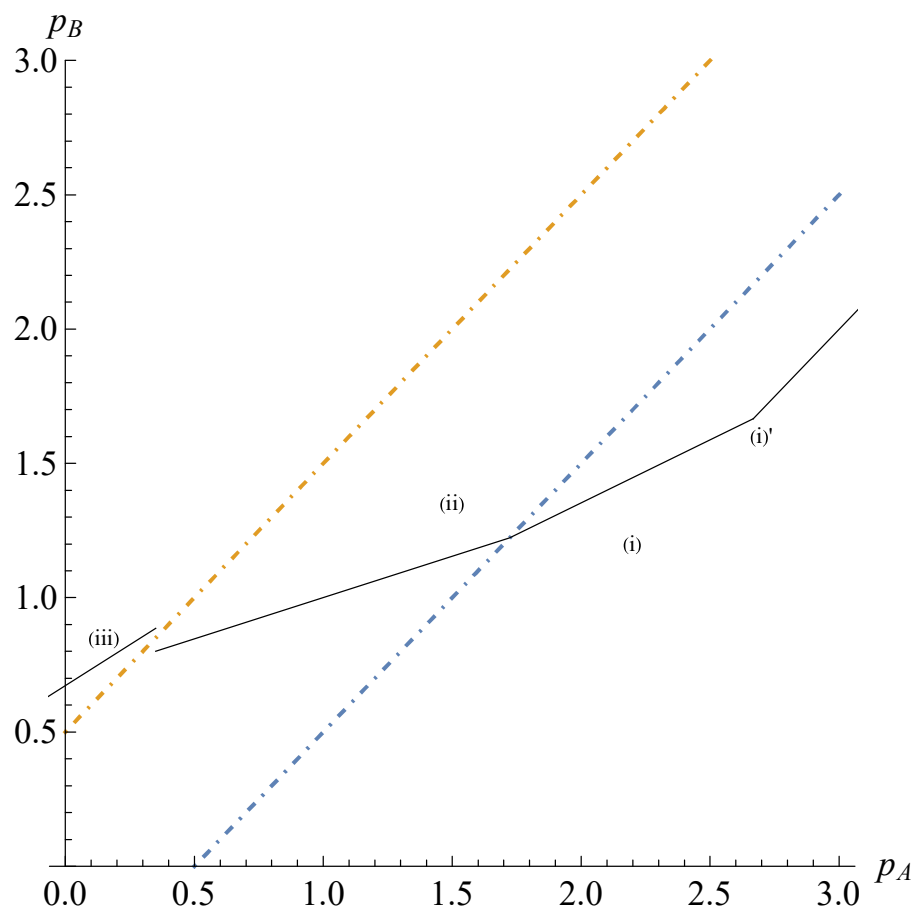




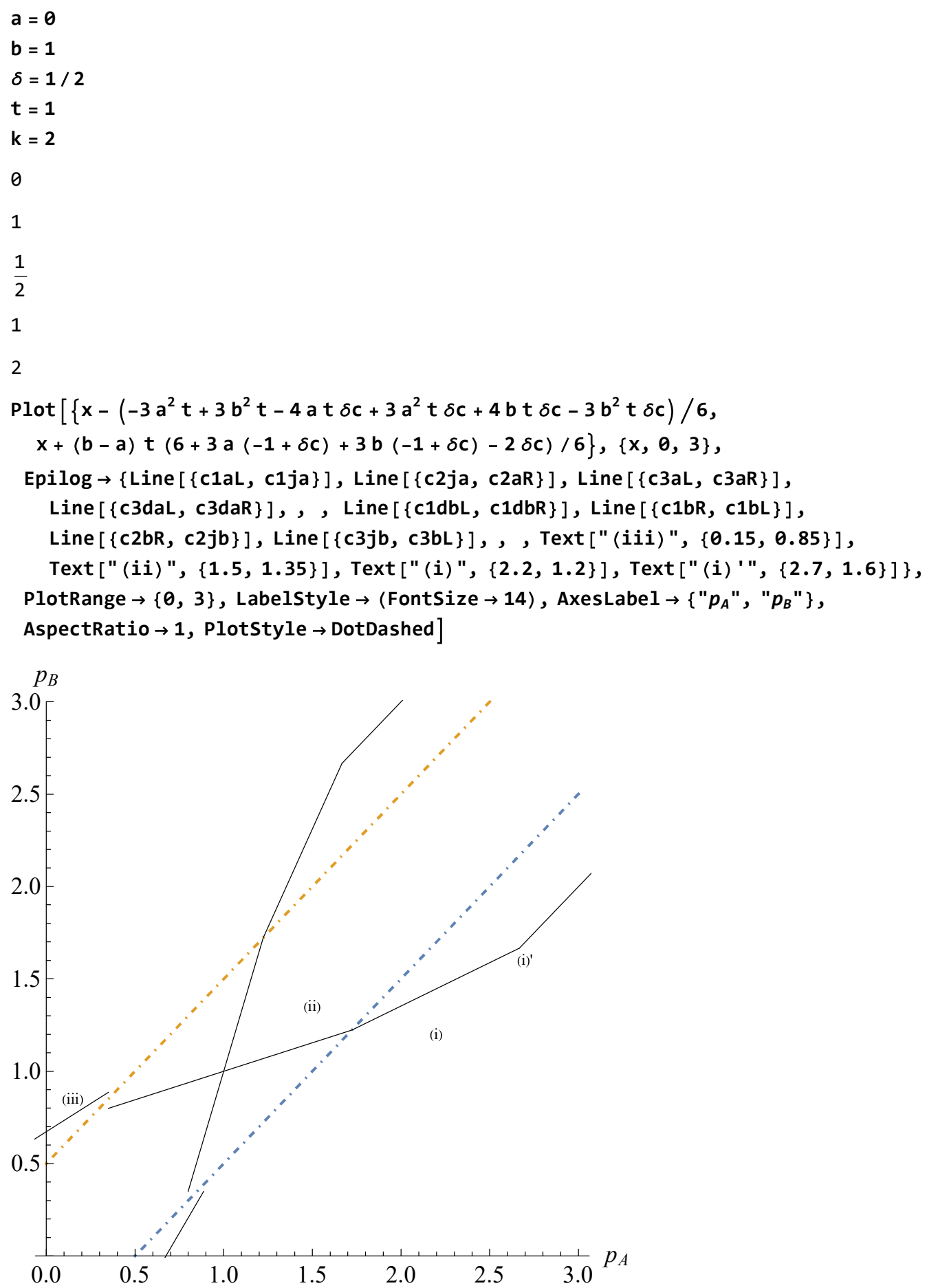




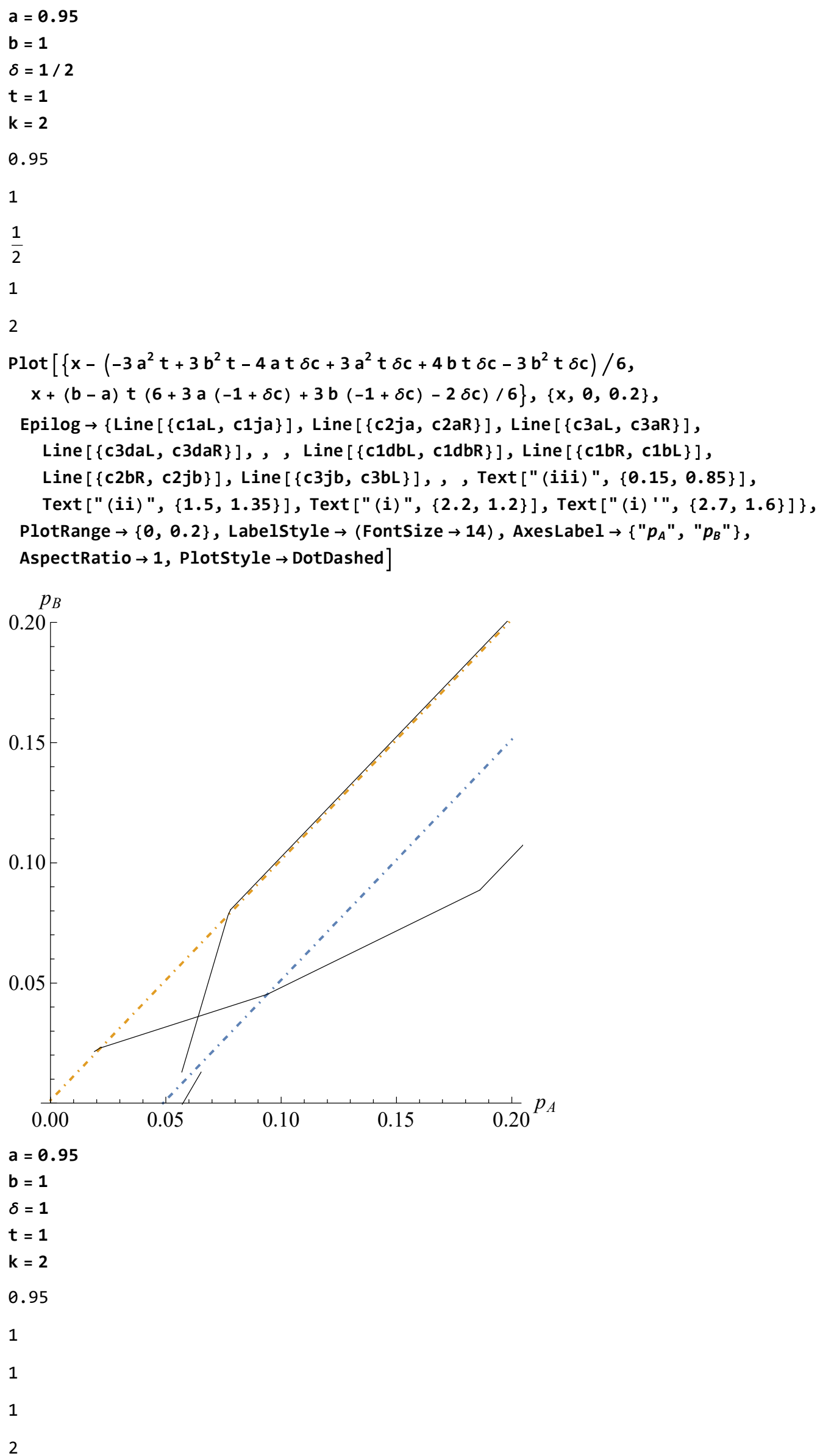




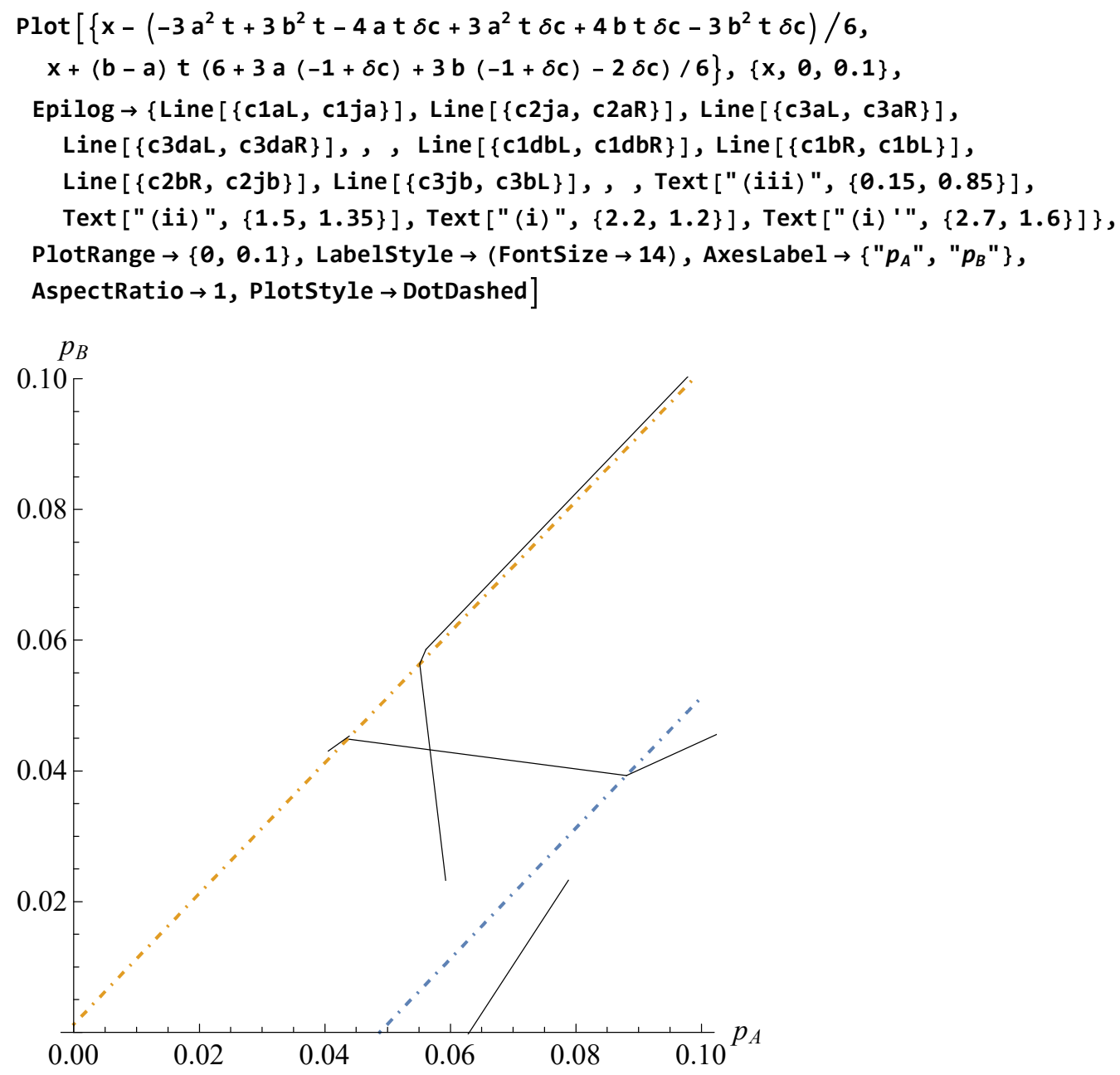

\section{Clear $[a, b, \delta, t, k]$}

The above examples suggest that the pricing equilibrium is possible only in (Case ii). We now show that it is indeed the case for general values of $(a, b, \delta, t, k)$. To this end, we use the reaction functions corresponding to (Case ii), find the intersection of the two reactions functions, and show that the intersection point is always in (Case ii) for all values of $\delta$.

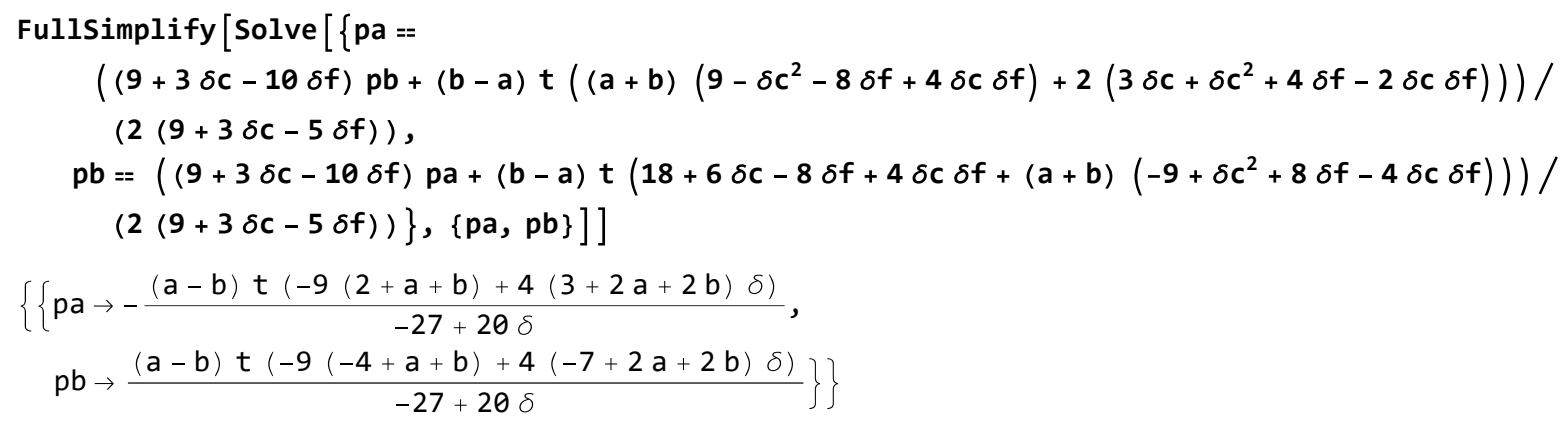

We can easily show that the derived intersection is always in (Case ii).

We need to show that the intersection is stable even when we consider the reaction functions outside (Case ii). 
From the result, if there is an intersection between the reaction functions of Firms A and B in (Case ii), the equilibrium $p_{B}$ is the following:

$E q p_{B}: \frac{(a-b) t(-9(-4+a+b)+4(-7+2 a+2 b) \delta)}{-27+20 \delta}$

We rewrite the jump point of Firm A's reaction function (c1ja):

c1ja

$$
\begin{aligned}
& \left\{\frac{1}{180(9+5 \delta)}(-a+b) t\left(160 \delta(9+5 \delta)+3(a+b)\left((21-10 \delta)(9+5 \delta)+(9+10 \delta) \sqrt{81-25 \delta^{2}}\right)\right),\right. \\
& \left.\frac{1}{9 \theta}(-a+b) t\left(80 \delta-3(a+b)(9+5 \delta)+3(a+b) \sqrt{81-25 \delta^{2}}\right)\right\}
\end{aligned}
$$

If the value of $p_{B}$ in the jump point is smaller than the equilibrium $p_{B}$ in (Case ii), the equilibrium point is stable. The difference between them is

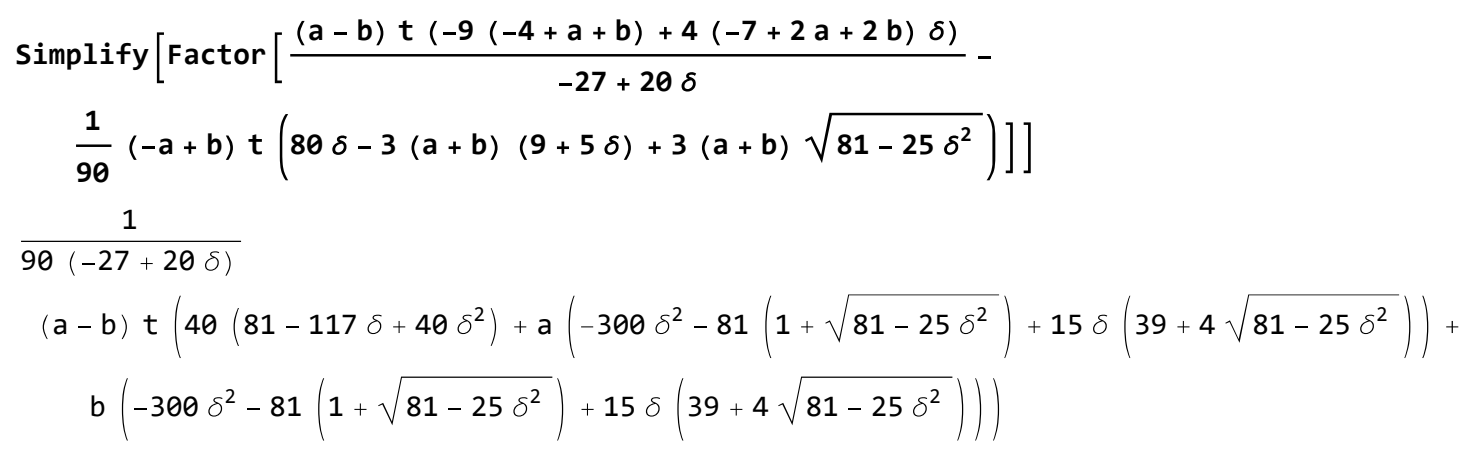

We arrange it, and obtain

$$
\frac{1}{90(27-20 \delta)}(\mathrm{b}-\mathrm{a}) \mathrm{t}\left(40(9-5 \delta)(9-8 \delta)-3(\mathrm{a}+\mathrm{b})\left((9-5 \delta)(3-2 \theta \delta)+(27-20 \delta) \sqrt{81-25 \delta^{2}}\right)\right)
$$

The sign of this value just depends on $\delta$ and $\mathrm{g} \equiv \mathrm{a}+\mathrm{b}$.

The value within the largest parentheses is linear in $\mathrm{g}(=\mathrm{a}+\mathrm{b})$.

This value when $\mathrm{g}=0$ is $40(9-5 \delta)(9-8 \delta)$, which positive.

We calculate this value when $\mathrm{g}=2$, and obtain

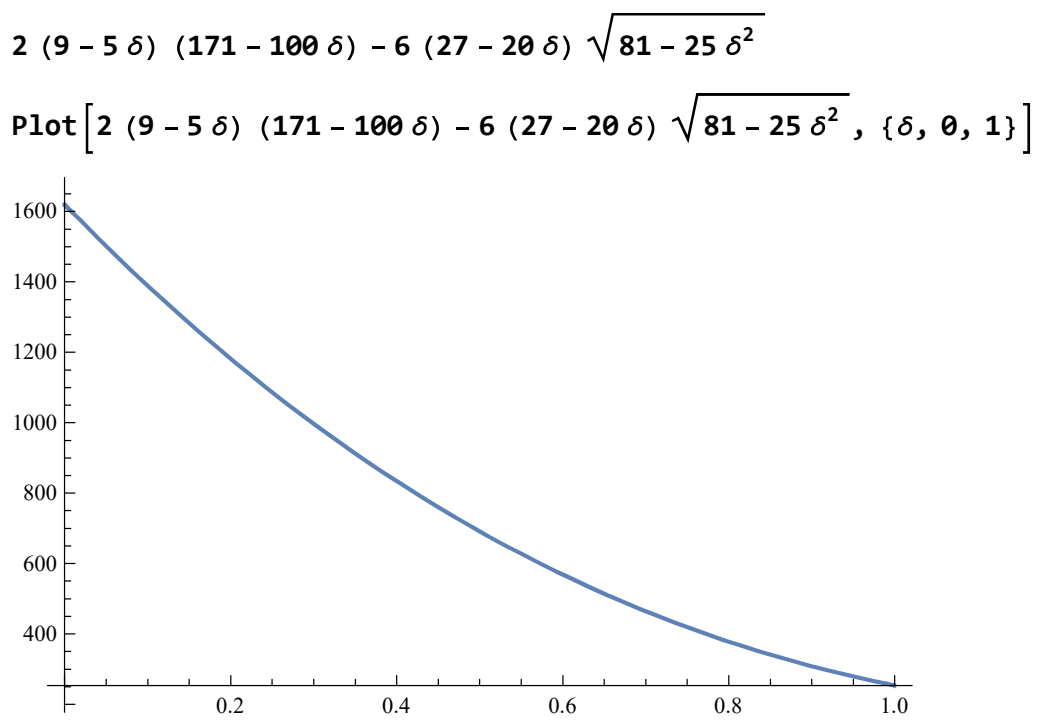

From the intersection of the reaction functions in (Case ii), the equilibrium $p_{A}$ is the following:

Eq pa : $-\frac{(a-b) t(-9(2+a+b)+4(3+2 a+2 b) \delta)}{-27+20 \delta}$ 
We rewrite the jump point of Firm B's reaction function (c3jb):

c3jb

$$
\begin{aligned}
& \left\{\frac{1}{90}(-a+b) t\left(-54+50 \delta+3(a+b)(9+5 \delta)+3(2-a-b) \sqrt{81-25 \delta^{2}}\right),\right. \\
& \frac{1}{180(9+5 \delta)}(-a+b) t(90(18(1+\delta)-(a+b)(9+5 \delta))+ \\
& \left.\left.\quad(9+10 \delta)\left(-54+50 \delta+3(a+b)(9+5 \delta)-3(-2+a+b) \sqrt{81-25 \delta^{2}}\right)\right)\right\}
\end{aligned}
$$

If the value of $p_{A}$ in the jump point is smaller than the equilibrium $p_{A}$ in (Case ii), the equilibrium point is stable. The difference between them is

$$
\begin{aligned}
& \text { Simplify }\left[\text { Factor } \left[-\frac{(a-b) t(-9(2+a+b)+4(3+2 a+2 b) \delta)}{-27+20 \delta}-\right.\right. \\
& \left.\left.\frac{1}{90}(-a+b) t\left(-54+50 \delta+3(a+b)(9+5 \delta)+3(2-a-b) \sqrt{81-25 \delta^{2}}\right)\right]\right] \\
& \frac{1}{90(-27+20 \delta)}(a-b) t\left(2\left(500 \delta^{2}-81\left(-19+\sqrt{81-25 \delta^{2}}\right)+15 \delta\left(-117+4 \sqrt{81-25 \delta^{2}}\right)\right)+\right. \\
& \quad a\left(300 \delta^{2}+81\left(1+\sqrt{81-25 \delta^{2}}\right)-15 \delta\left(39+4 \sqrt{81-25 \delta^{2}}\right)\right)+ \\
& \text { b } \left.\left(300 \delta^{2}+81\left(1+\sqrt{81-25 \delta^{2}}\right)-15 \delta\left(39+4 \sqrt{81-25 \delta^{2}}\right)\right)\right)
\end{aligned}
$$

We arrange it, and obtain

$$
\begin{aligned}
& \frac{(b-a) t}{90(27-20 \delta)}\left(2\left((9-5 \delta)(171-100 \delta)-(81-60 \delta) \sqrt{81-25 \delta^{2}}\right)+\right. \\
& \left.3(a+b)\left((9-5 \delta)(3-20 \delta)+(27-20 \delta) \sqrt{81-25 \delta^{2}}\right)\right)
\end{aligned}
$$

The sign of this value just depends on $\delta$ and $\mathrm{g} \equiv \mathrm{a}+\mathrm{b}$.

The value within the largest parentheses is linear in $\mathrm{g}(=\mathrm{a}+\mathrm{b})$.

This value when $\mathrm{g}=0$ is $2\left((9-5 \delta)(171-100 \delta)-(81-60 \delta) \sqrt{81-25 \delta^{2}}\right)$, which positive.

We calculate this value when $\mathrm{g}=2$, and obtain $40(9-5 \delta)(9-8 \delta)$, which is positive.
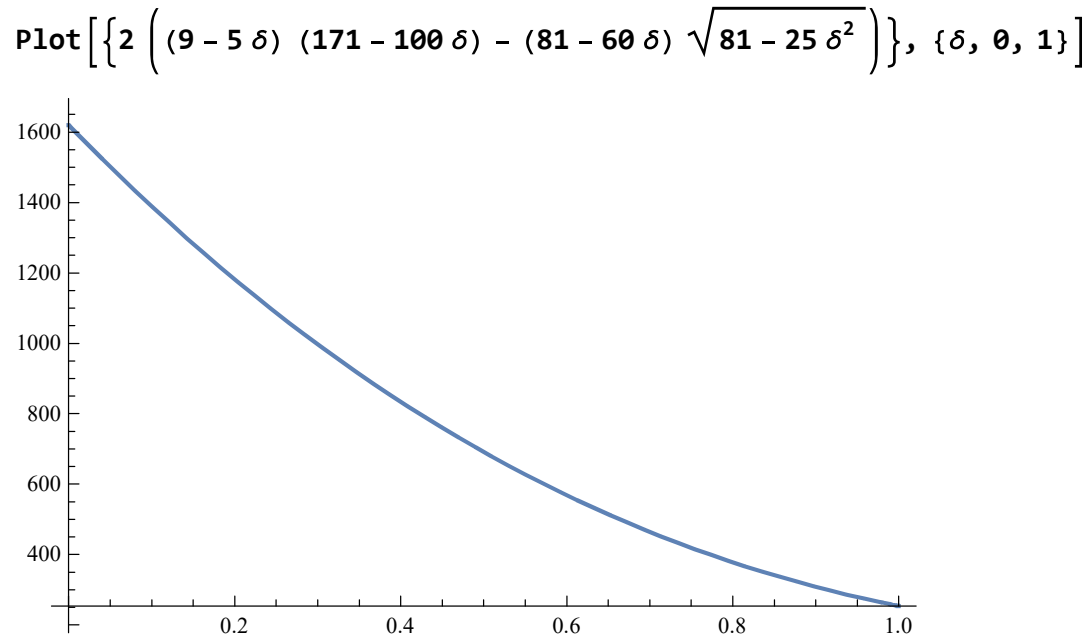

We have shown that the equilibrium is given by $a=0, b=1$ for all values of $\delta$. The rest of Proposition 2 follows by substituting $\mathrm{a}=0, \mathrm{~b}=1, \delta_{f}=\delta$ and $\delta_{c}=0$ into relevant prices and the locations of marginal consumers in the two periods. 


\section{The following discussion is related to Proposition 3.}

Here, we expand the range of $g(=a+b)$ from -1 to 3 .

Check the equilibrium locations in which the locations of the firms are restricted within the range $[-1 / 2,3 / 2]$.

$\frac{1}{90(27-20 \delta)}(\mathrm{b}-\mathrm{a}) \mathrm{t}\left(40(9-5 \delta)(9-8 \delta)-3(\mathrm{a}+\mathrm{b})\left((9-5 \delta)(3-20 \delta)+(27-20 \delta) \sqrt{81-25 \delta^{2}}\right)\right)$

The sign of this value just depends on $\delta$ and " $a+b$ ".

Here we define $g \equiv a+b$. The value within the largest parentheses is linear in $g(=a+b)$.

This value when $\mathrm{g}=-1$ is

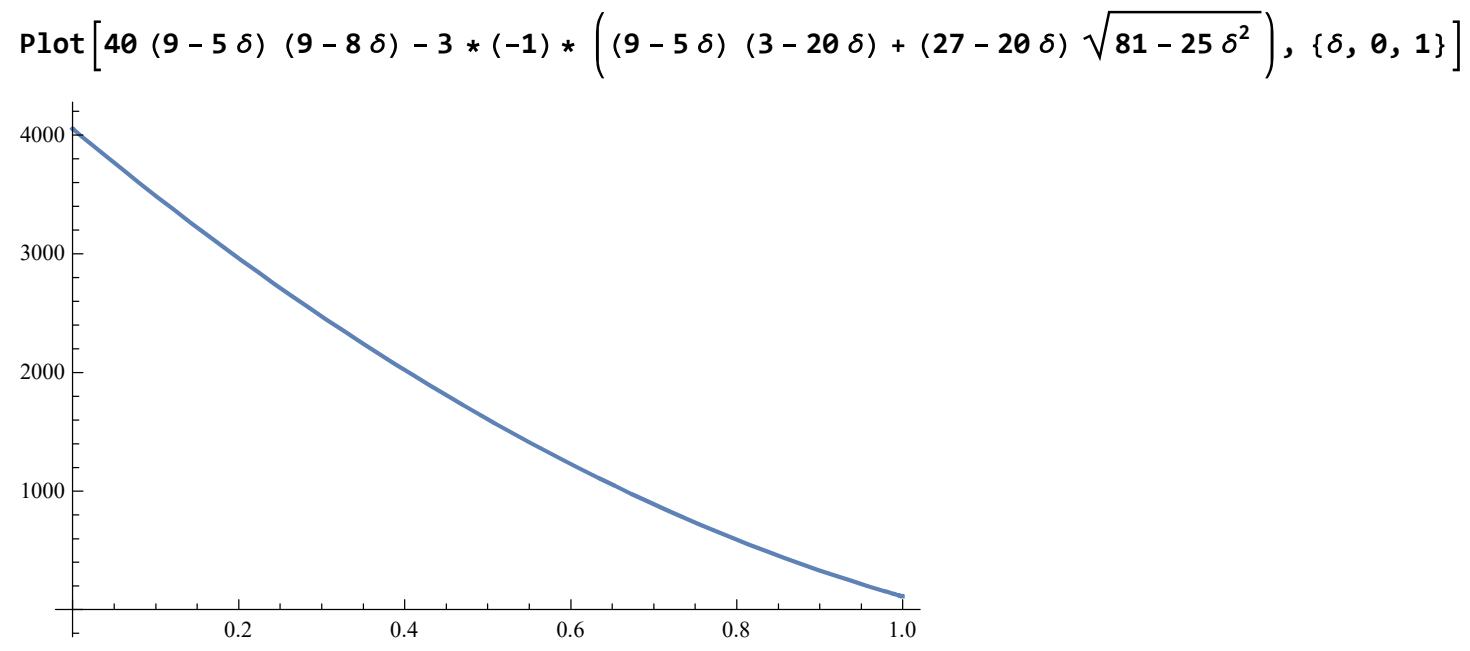

We calculate this value when $\mathrm{g}=3$, and obtain

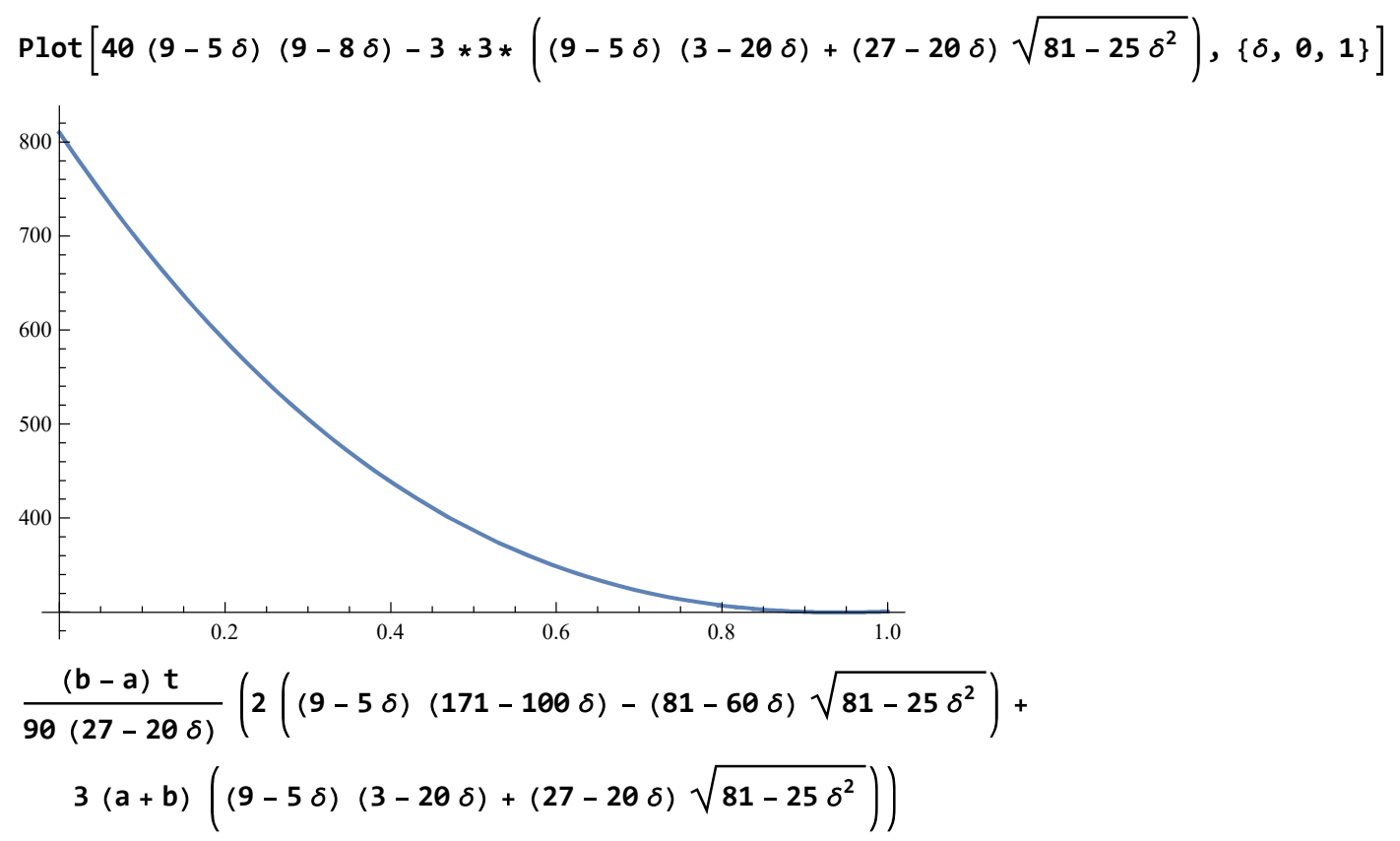

The sign of this value just depends on $\delta$ and " $a+b$ ".

Here we define $g \equiv a+b$. The value within the largest parentheses is linear in $g(=a+b)$.

This value when $\mathrm{g}=-1$ is 


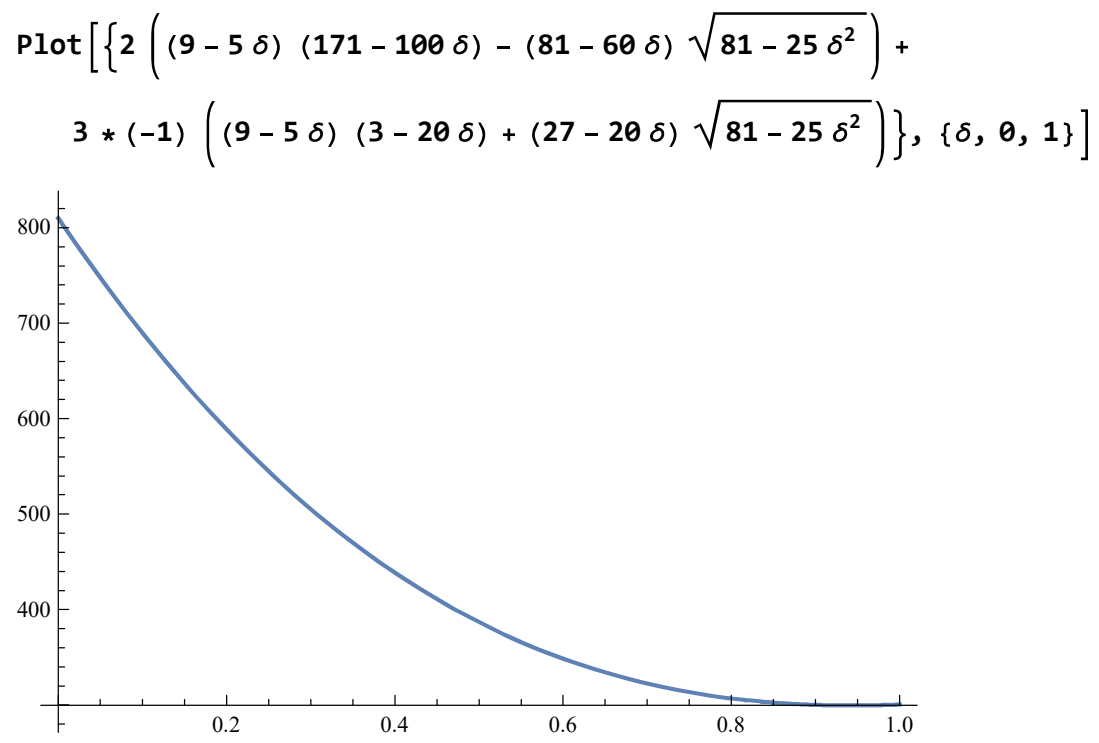

We calculate this value when $\mathrm{g}=3$ is

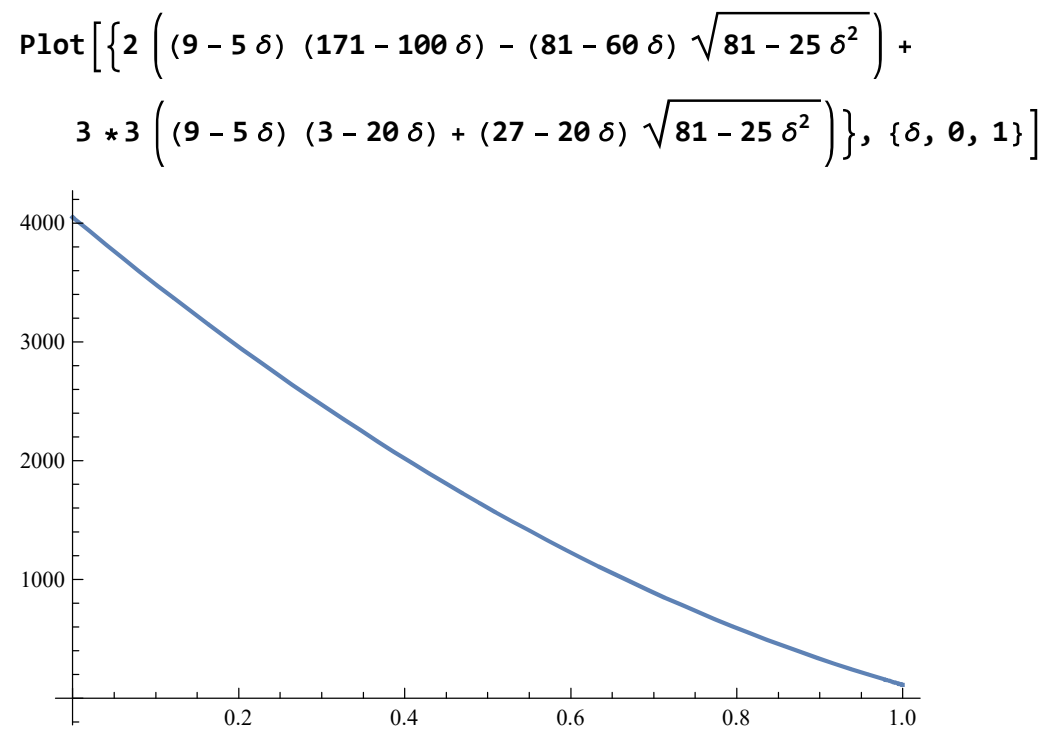

Check the equilibrium locations in which the locations of the firms are restricted within the range $[-1 / 2,3 / 2]$.

The following is the candidate locations of Firms A and B

$$
\begin{aligned}
& \text { a : }-\frac{81-99 \delta f+2 \theta \delta f^{2}}{12\left(27+9 \delta f-2 \theta \delta f^{2}\right)} \\
& \text { b : } 1+\frac{81-99 \delta f+2 \theta \delta f^{2}}{12\left(27+9 \delta f-2 \theta \delta f^{2}\right)}
\end{aligned}
$$

The profit of Firm A in case (ii) 


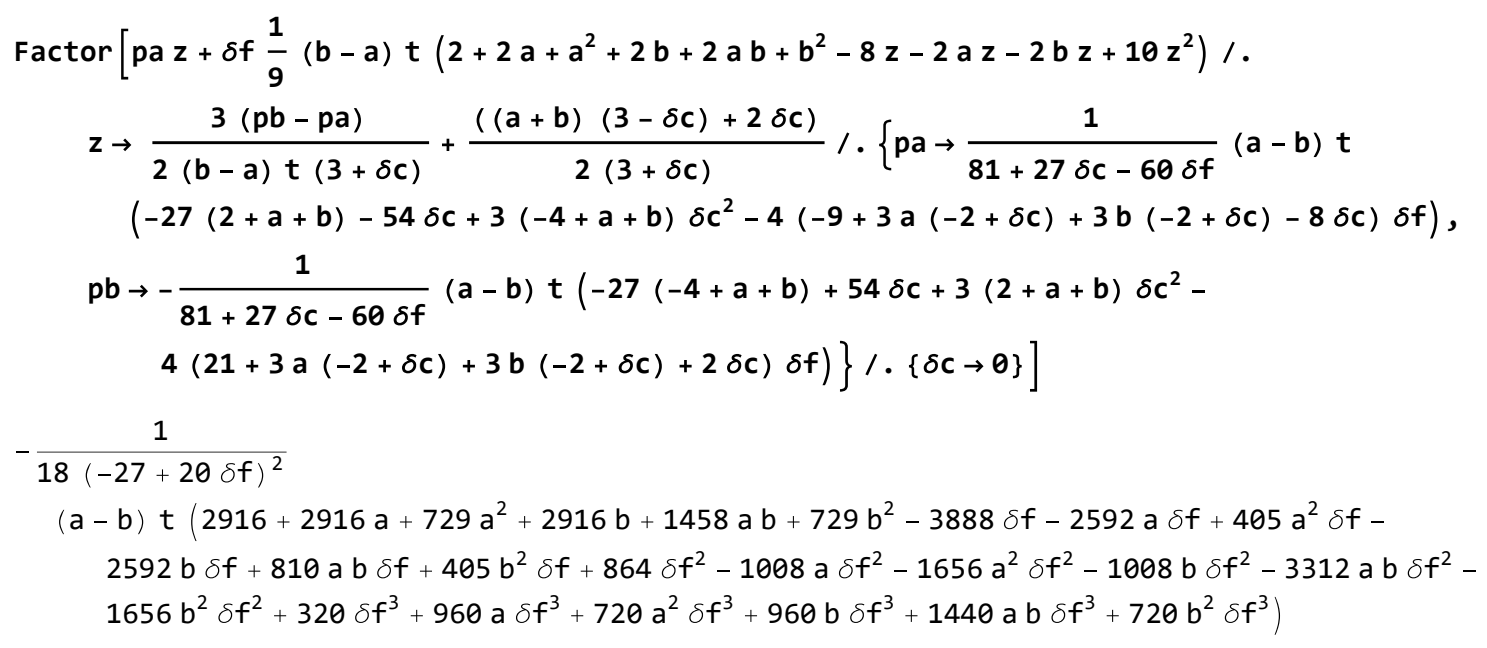

The first-order derivative of Firm A's profit with respect to $a$ is Factor $[D[\%, a]]$

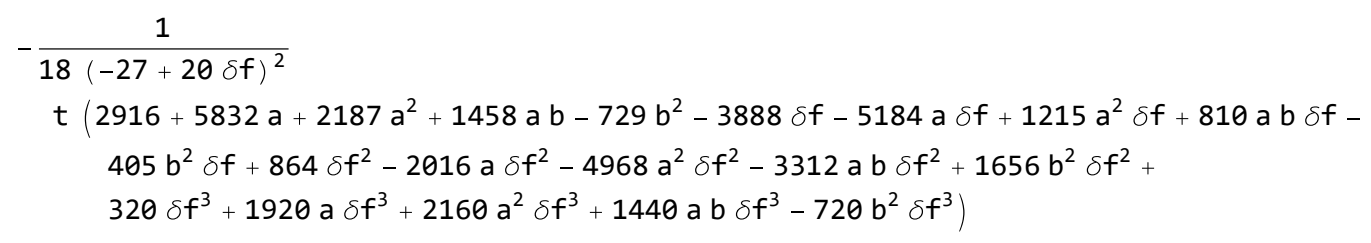

We substitute the candidate location of Firm B into the first-order derivative:

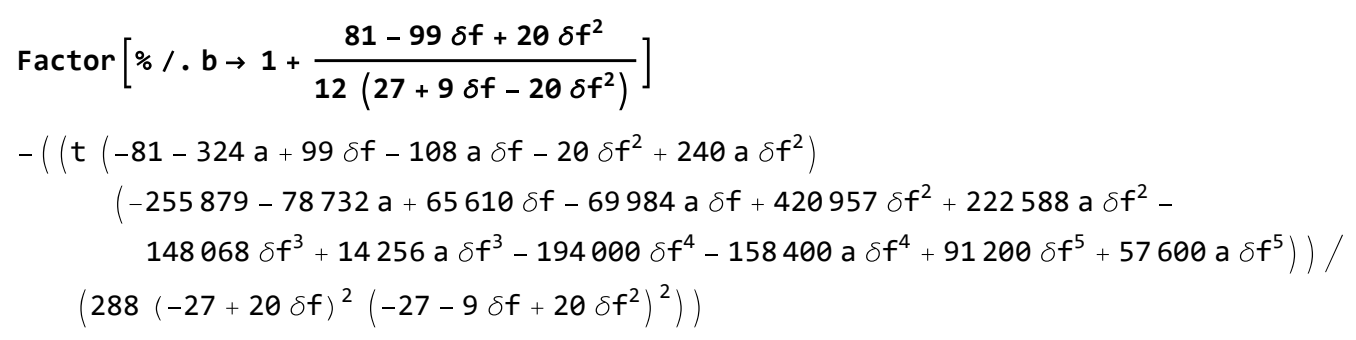

We derive $a$ which makes the above first-order derivative equal to zero.

Simplify [Solve $[\%=0, a]$ ]

$$
\begin{aligned}
& \left\{\left\{a \rightarrow \frac{81-99 \delta f+20 \delta f^{2}}{12\left(-27-9 \delta f+20 \delta f^{2}\right)}\right\},\right. \\
& \left\{a \rightarrow\left(255879-65610 \delta f-420957 \delta f^{2}+148068 \delta f^{3}+194000 \delta f^{4}-91200 \delta f^{5}\right) /\right. \\
& \left.\left.\quad\left(36\left(-2187-1944 \delta f+6183 \delta f^{2}+396 \delta f^{3}-4400 \delta f^{4}+1600 \delta f^{5}\right)\right)\right\}\right\}
\end{aligned}
$$

The former $a$ coincides with the candidate location of Firm A.

To check the sign of the first-order condition, we draw the values of $a$ just derived above. 


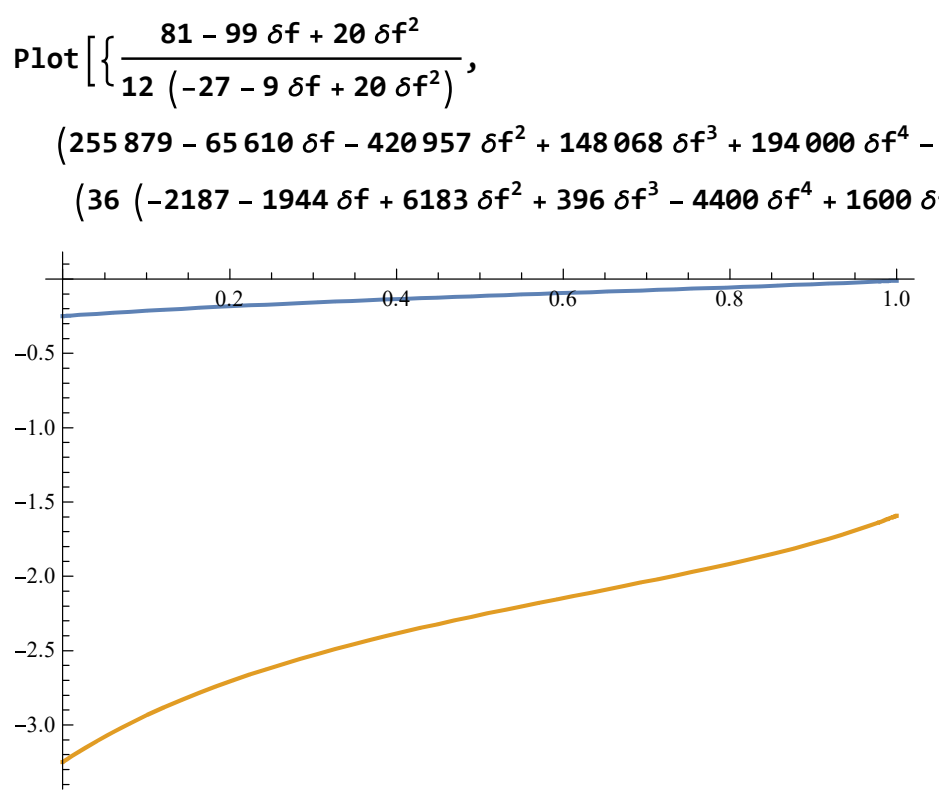

The first-order derivative with $b=1+\frac{81-99 \delta f+2 \theta \delta f^{2}}{12\left(27+9 \delta f-2 \theta \delta f^{2}\right)}$ is positive if $a<\frac{81-99 \delta f+2 \theta \delta f^{2}}{12\left(-27-9 \delta f+2 \theta \delta f^{2}\right)}$, otherwise it is negative. Therefore, $a=-\frac{81-99 \delta f+2 \theta \delta f^{2}}{12\left(27+9 \delta f-2 \theta \delta f^{2}\right)}$ is the best location for Firm A given that Firm B chooses $b=1+\frac{81-99 \delta \boldsymbol{f}+20 \delta \mathbf{f}^{2}}{12\left(27+9 \delta \boldsymbol{f}-2 \theta \delta f^{2}\right)}$.

The profit of Firm B in (Case ii)

$$
\begin{aligned}
& \text { Factor }\left[p b(1-z)+\delta f \frac{1}{9}(b-a) t\left(8-4 a+a^{2}-4 b+2 a b+b^{2}-8 z-2 a z-2 b z+10 z^{2}\right) /\right. \\
& \quad z \rightarrow \frac{3(p b-p a)}{2(b-a) t(3+\delta c)}+\frac{((a+b)(3-\delta c)+2 \delta c)}{2(3+\delta c)} / \cdot\left\{p a \rightarrow \frac{1}{81+27 \delta c-60 \delta f}(a-b) t\right. \\
& \quad\left(-27(2+a+b)-54 \delta c+3(-4+a+b) \delta c^{2}-4(-9+3 a(-2+\delta c)+3 b(-2+\delta c)-8 \delta c) \delta f\right), \\
& p b \rightarrow-\frac{1}{81+27 \delta c-60 \delta f}(a-b) t\left(-27(-4+a+b)+54 \delta c+3(2+a+b) \delta c^{2}-\right. \\
& \quad 4(21+3 a(-2+\delta c)+3 b(-2+\delta c)+2 \delta c) \delta f)\} / \cdot\{\delta c \rightarrow \theta\}] \\
& \quad \begin{array}{l}
18(-27+20 \delta f)^{2} \\
(a-b) t\left(11664-5832 a+729 a^{2}-5832 b+1458 a b+729 b^{2}-7452 \delta f+972 a \delta f+405 a^{2} \delta f+\right. \\
972 b \delta f+810 a b \delta f+405 b^{2} \delta f-7776 \delta f^{2}+7632 a \delta f^{2}-1656 a^{2} \delta f^{2}+7632 b \delta f^{2}-3312 a b \delta f^{2}- \\
\left.1656 b^{2} \delta f^{2}+5120 \delta f^{3}-3840 a \delta f^{3}+720 a^{2} \delta f^{3}-3840 b \delta f^{3}+1440 a b \delta f^{3}+720 b^{2} \delta f^{3}\right)
\end{array}
\end{aligned}
$$

The first-order derivative of Firm B's profit with respect to $b$ is

$$
\text { Factor }[D[\%, b]]
$$

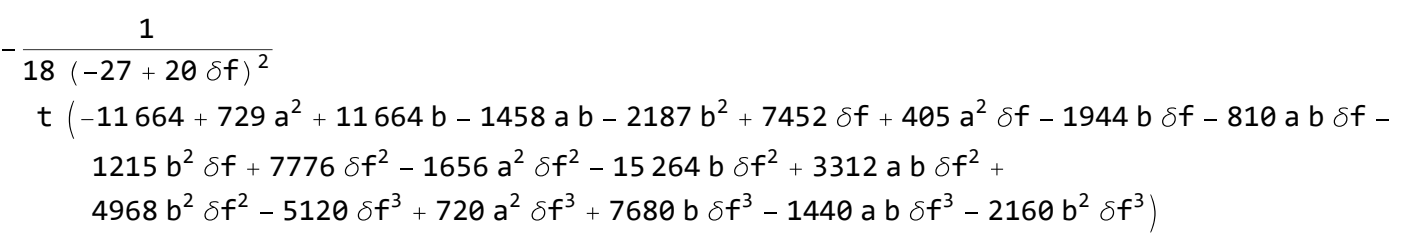

We substitute the candidate location of Firm A into the first-order derivative: 


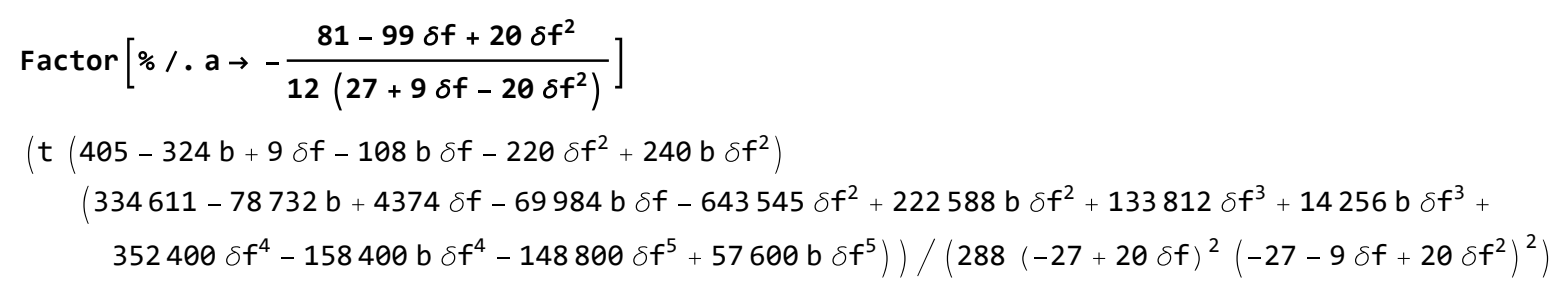

We derive a which makes the above first-order derivative equal to zero:

Simplify [Solve $[\%=0, b]$ ]

$$
\begin{aligned}
& \left\{\left\{b \rightarrow \frac{405+9 \delta f-220 \delta f^{2}}{324+108 \delta f-240 \delta f^{2}}\right\},\right. \\
& \left\{b \rightarrow-\left(\left(334611+4374 \delta f-643545 \delta f^{2}+133812 \delta f^{3}+352400 \delta f^{4}-148800 \delta f^{5}\right) /\right.\right. \\
& \left.\left.\left.\quad\left(36\left(-2187-1944 \delta f+6183 \delta f^{2}+396 \delta f^{3}-4400 \delta f^{4}+1600 \delta f^{5}\right)\right)\right)\right\}\right\}
\end{aligned}
$$

We rewrite the former $b$ :

$$
\begin{aligned}
& \text { Factor }\left[\frac{405+9 \delta f-220 \delta f^{2}}{324+108 \delta f-240 \delta f^{2}}-1\right] \\
& -\frac{81-99 \delta f+20 \delta f^{2}}{12\left(-27-9 \delta f+20 \delta f^{2}\right)}
\end{aligned}
$$

The former $b$ is

$b: 1+\frac{81-99 \delta f+20 \delta f^{2}}{12\left(27+9 \delta f-20 \delta f^{2}\right)}$

The former $b$ coincides with the candidate location of Firm B.

To check the sign of the first-order derivative, we draw the values of $b$ just derived above.

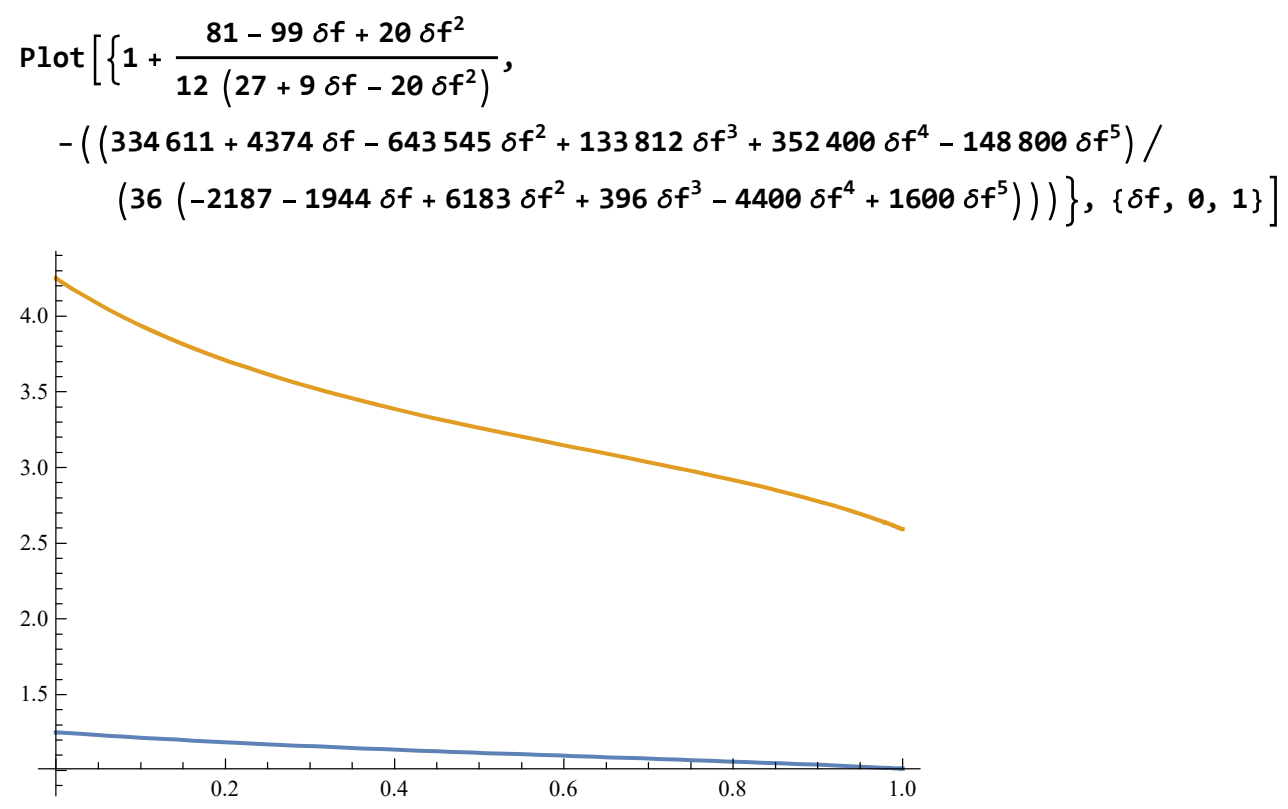

The first-order derivative with $a=-\frac{81-99 \delta f+2 \theta \delta f^{2}}{12\left(27+9 \delta f-2 \theta \delta f^{2}\right)}$ is positive if $b<1+\frac{81-99 \delta f+2 \theta \delta f^{2}}{12\left(27+9 \delta f-2 \theta \delta f^{2}\right)}$, otherwise it is negative. Therefore, $b=1+\frac{81-99 \delta f+2 \theta \delta f^{2}}{12\left(27+9 \delta f-2 \theta \delta f^{2}\right)}$ is the optimal location choice for Firm B given that Firm A chooses $a=-\frac{81-99 \delta f+20 \delta f^{2}}{12\left(27+9 \delta f-2 \theta \delta f^{2}\right)}$. 